\title{
9th Canadian Immunization Conference
}

\author{
Québec City Convention Centre, \\ Quebec City, Quebec \\ December 5 to 8, 2010
}

SPECIAL FEATURE
POSTER SUMMARIES FROM
FIRST NATIONS COMMUNITIES

There are many barriers experienced by front-line health care providers related to vaccine implementation, delivery and uptake in the various First Nations communities across Canada. Each region was invited to provide summaries of grass roots approaches in their communities to address some of these issues. These summaries highlight the experiences of immunization providers, while providing an awareness of and appreciation for the context of immunization as it relates to First Nations.

\section{P153 \\ PARTNERSHIPS, PANDEMIC PLANNING AND DELIVERY OF MASS IMMUNIZATION CLINICS IN ESGENÔOPETITG FIRST NATION}

Amy Schofield, Laurie Nicholas, Ginette Pellerin, Jeanne Comeau

BACKGROUND: Esgenôopetitg First Nation is located 32 kilometers northeast of the City of Miramichi, New Brunswick. It is the third largest First Nation in New Brunswick, with an on-reserve population of approximately 1190 and an off-reserve population of approximately 439. Also known as Burnt Church, it is a Mi'gmaq community situated within the Mirimachi Public Health area of Horizon Health Network.

In the past 3-5 years, Esgenôopetitg First Nation has developed and sustained strong relationships with Mirimachi Public Health Services in health emergency preparedness. In the pre-H1N1 period, prior to May 2009, the on-reserve elementary school was identified as one of the sites for mass immunization clinics for the geographic area.

PURPOSE: To demonstrate how partnerships based on trust and open effective communication between Horizon Health Authority, in particular Mirimachi Public Health Services and Esgenôopetitg First Nation influenced H1N1 community awareness, planning and implementation of the mass immunization clinic amid an evolving and at times a challenging environment. Building on the "informal" relationships of the previous 3-5 years, the Esgenôopetitg First Nation was involved at all levels in clinic planning, logistics and clinic operations.

METHODS: More than 850 community members were immunized during the one day 8 hour clinic.

RESULTS: Many community members assumed leadership roles throughout the various immunization stations.

CONCLUSION: There are many partners in pandemic planning; some of the success in the mass clinic in Esgenôopetitg First Nation may be attributed to the participation and support of the Chief and Council, trusting relationships with local public health; timely communication with key community members; participation and recognition of volunteers and rapid adjustment to evolving situations.

\section{P154}

HOW COLLABORATION BETWEEN ESKASONI COMMUNITY HEALTH CENTRE AND PUBLIC HEALTH SERVICES, CAPE BRETON DISTRICT HEALTH AUTHORITY LED TO A SUCCESSFUL H1N1 IMMUNIZATION CAMPAIGN IN ESKASONI FIRST NATION

Judi Rutherford, Roselita Herney, Judy Kelley, Anne Romard

BACKGROUND: Eskasoni First Nation is located in Cape Breton,
Nova Scotia. It is approximately 45 minutes from the nearest city, Sydney. Nurses in Eskasoni First Nation knew that H1N1 vaccine uptake would be low if clients had to travel off reserve to be immunized. Eskasoni Community Health Centre and Public Health Services, Cape Breton District Health Authority collaborated to provide immunization clinics on reserve.

PURPOSE: To demonstrate how the strong collaborative relationship between Eskasoni Community Health Centre and Public Health Services, Cape Breton District Health Authority led to successful clinics and high H1N1 immunization coverage rates.

RESULTS: Eskasoni Community Health Centre collaborated with Public Health Services months in advance to plan immunization clinics. While staff from the Eskasoni Community Health Centre took the lead in the actual clinics, Public Health Services provided invaluable guidance and logistical support. The community and leadership supported this strategy and, as a result, H1N1 coverage rates in Eskasoni First Nation exceeded all national and provincial target rates. The coverage rate for children under 18 years was over $95 \%$.

CONCLUSION: Much of the success of Eskasoni's H1N1 clinics is attributed to the strong collaborative relationship with Public Health Services that has been developed over several years. This relationship has been recognized as a necessary component to all population health programming that Memorandums of Understanding have been drafted to ensure lasting success.

\section{P155}

\section{H1N1 VACCINE CAMPAIGN A TREMENDOUS SUCCESS:} LESSONS LEARNED

Melanie Turpin, Illana Laderoute

BACKGROUND: The Fall 2009 Pikangikum H1N1 vaccine campaign was a tremendous success, prompting us to question why.

PURPOSE: Surprised and very pleased with the vaccine uptake, we decided to take a close look at the possible reasons for the success and how they could be applied to future campaigns and to our general, regularly scheduled immunization program.

METHODS: Group discussions amongst the nurses involved in the campaign and group and one-on-one discussions with the support staff who assisted with the campaign, community members and leadership members.

RESULTS: We discovered that there were many reasons for our success. These included community awareness of the vaccine and the swine flu, support from the chief and council, extra nurses dedicated to the immunization program, tremendous help from our support staff, dollars from Health Canada to cover the costs of items or resources needed for a successful campaign, and a stellar health promotion/health teaching effort that included radio shows, poster presentations, and on-line education. We also recognized the fact that we delivered a very culturally sensitive and culturally-appropriate campaign, giving much consideration to language, traditions, beliefs, socio-economic level, social/family structures, infrastructure and the history of our clients.

CONCLUSION: An active campaign that includes community awareness of the vaccine and swine flu, support of chief and band council, and additional staff and funds lead us to a very successful immunization blitz. In the future, we plan to continue our campaign of immunization awareness in the community by always involving the chief and council, all the community members that were involved, and any other help that we can recruit. Positive attitudes with the campaign and the feeling of great achievement left us all with an appetite for even greater challenges and success in future. 


\section{P156}

INNOVATIVE IMMUNIZATION STRATEGY IN A FIRST NATIONS COMMUNITY

\section{Karen D Martin}

BACKGROUND: Immunization rates for on-reserve children are 20 percent

lower than other children in Canada.

To provide immunization opportunities based on the guideline set out in the Canadian Immunization Guide 7th Edition.

Immunization services should be readily available.

Providers should use all clinical opportunities to screen for needed vaccines and, when indicated, to vaccinate.

Providers should administer all vaccine doses for which a recipient is eligible at the time of each visit.

Providers should ensure that all vaccinations are accurately and completely recorded.

Community members and agencies work together to deliver a Baby Show at the annual fall fair. Six Nations Public Health Office, Six Nations Health Services, Six Nations Healthy Babies/Healthy, Six Nations Agricultural Society, Six Nations Fair Board, and Six Nations community volunteers.

PURPOSE: To improve the immunization coverage rate of on-reserve First Nations children younger than four years of age.

OPPORTUNITY: On average, 50 to 60 children register for the baby show every year. All of these children register at the Six Nations Public Health Office. Each visit entails a Growth \& Developmental Assessment, Nipissing Screen, Immunization review, all vaccinations given as needed and referred as needed i.e dietician, dental, speech, physician.

CONCLUSION: Registering children for the baby show is an effective means of ensuring children complete their primary series of immunization.

\section{P157 \\ PANDEMIC INFLUENZA - EXPERIENCE OF FIRST NATIONS IN MANITOBA}

Caroline Bercier, Gloria Rach, Tina Linklater

PURPOSE: The goal of developing the resource (DVD) was to document the experience of the HINI Influenza Pandemic in First Nation Communities. In the initial response to the H1N1 Pandemic, there was a need for documentation of previous experience of First Nation communities to past pandemic(s) both for planning and raising awareness.

METHODS: Interviews were conducted with various First Nations communities with front-line health service providers, community members and individuals impacted directly by the H1N1 virus.

RESULTS: The development of the DVD resource not only documents the personal experiences, but also provides an invaluable education tool for both planning and mobilizing communities in their response to the pandemic.

CONCLUSION: By documenting the Manitoba First Nations experiences, this resource can be utilized as a teaching tool for emergency response planning such as for Pandemic Planning.

\section{P158}

\section{MASS PH1N1 AND SEASONAL FLU IMMUNIZATION CLINICS IN 3 NORTHERN ALBERTA FIRST NATIONS COMMUNITIES IN 2009}

Sandra Caharel, Joseph Redhead, Dee Dee Skoreyko, Ruth Richardson

PURPOSE: To demonstrate the development of the Mass H1N1 and Seasonal Flu Immunization Clinics for the three bands in the Western Cree Tribal Council - Duncan's First Nations, Horse Lake First Nations and Sturgeon Lake Cree Nations.

DESIGN AND APPROACH: The development of the Mass Immunization Clinics came about following the Nursing Model: assessment, planning, implementation and evaluation. The Nursing Model was then adapted into the Medicine Wheel Model, which is used by many Aboriginal Nations. The process for collaboration with all communities will be described and a copy of the original map for the layout for the immunization will be shown.
FINDINGS: Data will be shown demonstrating the comparison between the H1N1 and Seasonal Flu vaccine administration in the First Nations communities. What worked, what didn't and the obstacles faced at each stage of the game as the partners came together will be discussed.

ORIGINALITY/VALUE: The development of a framework for implementing a Mass Immunization Clinic in First Nations communities can be useful in providing a large group of people a service that will benefit the community, as well as each individual member. The data for the uptake of H1N1 and Seasonal Flu vaccines give a clear understanding of where people viewed the importance of both vaccines. This information can guide planning for future Mass Immunizations clinics. This poster also demonstrates how all staff of the Health Care team as well as community members can come together for the betterment of all communities' members; from infants all the way up to the elders.

CONCLUSION: There was a marked difference in uptake for pH1N1 (ranging from 59\% to 111\%) and Seasonal flu (ranging from 21\% to 70\%) vaccines. Information from this analysis will guide planning for future Influenza immunization strategies.

\section{P159}

\section{MASS IMMUNIZATION ROLL OUT OF PH1N1 AND SEASONAL INFLUENZA IMMUNIZATION IN ALBERTA FIRST NATIONS}

Ruth Richardson, Wadieh Yacoub, Jeff Kresowaty

BACKGROUND: Seasonal influenza vaccine has been offered in FN communities in $\mathrm{AB}$ for many years, with approximately 15,000 doses administered over a 5 month period each year. In 2009, communities were asked to develop a plan to immunize their total population in 3 weeks. The total on-reserve population in Alberta is approximately 65,000.

STRATEGY: In 2009, targeted funding was made available to all communities for pandemic influenza planning activities and communities were asked to submit their mass immunization plan as well as emergency contact information by September 1, 2009.

COMMUNITY PLANNING: All 44 First Nations communities submitted draft mass immunization plans. These were reviewed and feedback was provided to community planners. Most communities incorporated nonhealth sector staff in their plans; communities with little or no nursing services teamed up with neighbouring communities to provide access to immunization.

REGIONAL PLANNING: The focus of regional planning for immunization was on measures to support the front-line staff including the following:

- Memorandum Of Agreement with the province for pandemic vaccine allocation

- Vaccine distribution to communities

- Supply availability and distribution

- Regional nursing travel team to assist communities as needed. RESULTS: $43 / 44$ communities offered $\mathrm{pH} 1 \mathrm{~N} 1$ vaccine the first week it was available - administering 27,588 doses. Many had tested their plan the previous week for seasonal flu vaccine. Total individuals immunized were $42,952(66 \%)$ and 25,707 (38\%) against pH1N1 and seasonal influenza, respectively.

CONCLUSION: The mass immunization planning and implementation carried out in First Nation communities in Alberta resulted in 66\% of the on-reserve population being immunized against pH1N1. The majority of communities plan to use a similar approach for seasonal influenza vaccine each year.

\section{P160 \\ IMPLEMENTING PANDEMIC INFLUENZA RESPONSE IN A FIRST NATION COMMUNITY IN ALBERTA}

Carolyn Henry, Darlene Richter, Ruth Richardson, Brent Whitall, Glenn Curtis

BACKGROUND: Influenza-like illness began in our community before there was vaccine available. We were able to institute a process to make antiviral medication available locally, and then successfully incorporated a mass immunization program once the vaccine was available. 
METHODS: Regular operations at the Health Centre were shut down in order to concentrate on the pandemic response for both influenza-like illness assessment service and mass immunization. Volunteers and CHRs assisted with documentation and traffic flow; home-care staff updated the list of the individuals with chronic conditions, including Elders: pH1N1, Flu and PPV23 vaccines were offered in a targeted strategy.

RESULTS: Total of 18 confirmed H1N1 cases: 5 with the first wave, 13 in the second wave. One hospitalization and no mortality was associated with H1N1.

31 courses of antivirals distributed; 1581 doses of seasonal and 2498 of pH1N1 influenza vaccines were administered. For pH1N1: overall $62 \%$ of the population was immunized, with $85 \%$ of 6 month to 4 years; $43 \%$ of $65+$ years; $98 \%$ of health care workers and $100 \%$ pregnant woman protected. For PPV23: $20 \%$ of $65+$ and $11 \%$ of the chronic clients were immunized.

DISCUSSION: In spite of running out of Seasonal Flu vaccine, we increased our coverage in comparison to previous years by $44 \%$. Staff worked very long hard hours and felt overwhelmed. They also felt guilty and frustrated that the regular program was not being done. People from off reserve came as they heard that our clinic was giving vaccine without long waits. Initially, we tried to accommodate them until we received the directive from FNIH to concentrate on our population, with some complaints received. While PPV23 was not the focus, the mass immunization provided the opportunity to update the list of the over 65 and those with chronic health conditions.

\section{P161}

\section{SIKSIKA FIRST NATION EXPERIENCE WITH SEASONAL AND} H1N1 INFLUENZA - 2009

\section{Kristin Robertson, Berna Moss, Ruth Richardson}

BACKGROUND: We propose to prepare a poster which will describe our seasonal Flu and H1N1 experience in the fall of 2009 which, due to extenuating circumstances, was to become a much bigger project than we ever imagined.

PURPOSE: Our location in southern Alberta on the Trans-Canada Highway and within easy access of population areas all around us resulted in a totally unexpected influx of non-nation H1N1 immunization seekers, some coming from as far as $200 \mathrm{~km}$. We will describe how we adapted to that.

METHODS: We partnered with all services in Siksika Health and established rosters, duties and provided breaks and healthy snacks for staff. For clients, we monitored the waiting lines, moving elders, pregnant women and large families to the front of the line. We established a Flu Response Unit (FRU) in conjunction with Emergency Services. This team consisting of a paramedic and a community health nurse responded to calls where influenza-like illnesses were described and eliminated many ambulance calls and emergency department (ER) visits.

CONCLUSIONS: We immunized over $80 \%$ of the community's population, not counting the large number of non-nation members. Through the establishment of the FRU, we eliminated a large number of ambulance calls and ER visits, and provided education and support to those families with ill members at home. Throughout the whole period, we experienced incredible teamwork from all members of Siksika Health Service staff, and from First Nations and Inuit Health.

\section{P162}

\section{SEABIRD ISLAND HEALTH}

\section{Elizabeth Point}

Our poster reviews our approach to immunizations. Seabird Health provides health services to ten communities in the Fraser region of British Columbia. Each community is unique and so we have some specific strategies for specific communities and some strategies that we use in all communities. Our average rate of immunizations is $95 \%$. General strategies include enhancing awareness of the need for immunizations, creating many opportunities for immunizations, tracking individuals so that we are aware of who is due for immunizations, and involving families in the immunization process. Specific strategies are used in communities that have some people who are reluctant to immunize. In these communities, we make sure that those who do not want immunizations feel respected. This ensures that they have ongoing contact with health professionals to build trust and provide education. In the mean time, we ensure that those who want immunizations can. We ensure confidentiality during the immunization process so that immunizations can be given discretely. Over time, we have found that our supportive approach has meant that fewer people are reluctant and the vast majority of community members receive the immunizations they need.

\section{P163}

\section{KTUNAXA NATION HEALTH}

\section{Myanne Peacock}

This poster examines the historical perspective of immunization within the Ktunaxa Community. It examines how immunization and vaccination has evolved through time from point of contact, through residential school up to the present. The purpose is to expose how these experiences have impacted the views around immunization and have either motivated individuals to vaccinate or created barriers in immunization uptake. My goal is that this poster will educate the public of the historical influences that have played a role in the current perception of immunization within a small rural Aboriginal community in British Columbia.

\section{P164 \\ TS'EWULHTUN HEALTH CENTRE OF THE COWICHAN TRIBES}

Leanne Kelly

In 2008, Cowichan Tribes became one of five Aboriginal sites to be involved in a vaccine study looking at the use of the new Infanrix-Hexa vaccine. This study was co-ordinated by $\mathrm{BC}$ Children's Hospital/UBC Vaccine Evaluation Center, GSK labs and community health providers in the province. Cowichan Tribes became involved because of an interest in the posed question, as to whether the vaccine would prove effective in providing protection to children of all backgrounds. The study aimed to compare the level of antibody response in children who are Aboriginal and those who are non-Aboriginal. The poster presentation will focus on the process that the health center staff and community undertook to prepare for and deliver this new vaccine. Issues of capacity, knowledge transfer, and sustaining resources are highlighted. Preliminary results of the study's implications for the Aboriginal communities are highlighted.

\section{ABSTRACTS AUTHOR INDEX}

Bercier C ................ P157

Caharel S ................. P158

Comeau J . . . . . . . . . . . . . . . . . P153

Curtis $G \ldots \ldots \ldots \ldots$. . . P160

Henry C ................ P160

Herney R . . . . . . . . . . . . . . . . P154

Kelley J .................... P154

Kelly L ..................... P164

Kresowaty J . . . . . . . . . . . . P159

Laderoute I ................ P155
Linklater T ................ P157

Martin K .................P156

Moss B .................P161

Nicolas L . . . . . . . . . . . . P153

Peacock M .....................P163

Pellerin G. ................. P153

Point E.................. P162

Rach G................P157

Redhead J. . . . . . . . . . . . . . P158

Richardson R ... P158,P159,P160,P161
Richter D . . . . . . . . . . . . . . . . P160

Robertson K. . . . . . . . . . . . . . P161

Romard A .............. P154

Rutherford J . . . . . . . . . . . P154

Schofield A . . . . . . . . . . . . . . P153

Skoreyko DD .............. P158

Turpin M ................ P155

Whitall B ................ P160

Yacoub W.................P159 
ORAL PRESENTATIONS

\section{O01}

IMPROVING HPV VACCINE COVERAGE RATE WITH CATCH-UP CLINICS IN TORONTO: PARENTS TELL US WHY THEY ARE IMPORTANT

H Meghani, Dubey, O Kadri, K Beckermann, J Cameron,

A Mathur

Toronto, ON

BACKGROUND: In August 2007 the Government of Ontario announced a human papillomavirus (HPV) vaccine campaign beginning September 2007 for grade 8 females. Vaccine was only available through public health clinics. The provincial HPV vaccination rate in the 2007/2008 academic session was 58\%, lower than other schoolbased programs. Catch-up clinics were an opportunity to get vaccinated later in the school year if a parent changed their mind.

OBJECTIVE: To assess factors that influenced parental decisions around HPV vaccination of their daughters.

METHODS: A cross-sectional survey of parents of grade 8 females attending Toronto Public Health (TPH) HPV catch-up clinics in 2008 was conducted. Parents were asked to complete a standardized selfadministered questionnaire. Univariate statistics were performed with SPSS to assess demographic characteristics of participants and parental reasons to vaccinate their daughters.

RESULTS: A total of 262 parents were recruited for the survey. The major reasons for receiving the vaccine were to prevent cervical cancer $(66.5 \%)$ and to protect their daughter's health (25.0\%). Reasons for attending catch-up clinics included absenteeism from school on the initial clinic day $(48.2 \%)$ and change of initial decision to not vaccinate $(23.3 \%)$. Parents who changed their mind about vaccinating their daughters cited the following reasons: recommendation from their doctor $(41.0 \%)$, more time to make a decision $(21.3 \%)$, recommendation from a friend/family (14.8\%), and more scientific information about the vaccine $(14.8 \%)$.

CONCLUSION: Catch-up clinics provided an important opportunity to improve access and enhance HPV vaccination rates among grade 8 females in Toronto. The influence of physicians and additional time for decision-making played a role in catch-up clinic attendance. Future vaccine campaigns should engage primary care physicians and provide parents time to decide or provide opportunities to receive the vaccine at a later date.

\section{2 \\ VACCINE ADMINISTRATION ERRORS BY NURSES IN PUBLIC HEALTH}

\section{F Hemming ${ }^{1}$, C Demarinis ${ }^{1}$, J McCrea ${ }^{2}$, L Jarvos ${ }^{3}$}

${ }^{1}$ Delta, BC; ${ }^{2}$ Maple Ridge, BC; ${ }^{3}$ Mission, BC

BACKGROUND: One of the Public Health Agency of Canada's Immunization Competencies is to prepare and administer immunization agents correctly. Reviewing errors, identifying trends, and recommending strategies to decrease these errors is a way to increase safety in immunization clinics.

PURPOSE: To decrease vaccine administration errors by:

- identifying common errors

- determining if there are any system processes that are contributing to errors

- ensuring providers are appropriately educated and trained to deliver immunization services and that training is up-to-date

- recommending ways to decrease common errors in the future (e.g. education, change to orientation information, change to system processes).

METHODS: The Fraser Health Communicable Disease Team collected data on vaccine administration errors made by nurses in Public Health for 2008 and 2009 to review trends in the following:
- vaccine errors

- possible nursing practice issues

- errors related to immunization settings (e.g. mass/school clinics vs. Health Unit setting)

- experience level of the nurse (e.g. novice vs. experienced nurses)

- clinic flow processes that might contribute to error rates

- knowledge gaps leading to higher error rates; e.g., administration technique, 7 rights to administration, informed consent process

RESULTS: The Fraser Health Communicable Disease team received 262 vaccine administration error reports between January 2008 and December 2009. There were approximately 250 full-time nurse equivalents working out of 18 Health Units during the same period. The most common errors were due to vaccines being given off schedule $(31.4 \%)$, the incorrect product being used $(27.3 \%)$, and extra or missed doses due to lack of immunization records $(24.0 \%)$.

CONCLUSION: Results suggest differences in error rates across geographic boundaries in Fraser Health, as well as by delivery setting. Further investigation will be conducted using qualitative analyses to determine the impact of clinic flow and nursing knowledge gap on errors rates. Information from these analyses will be used to develop recommendations, such as additional education or systems change, to decrease error rates.

\section{O03}

\section{IMMUNIZATION COVERAGE AMONG ONE AND TWO YEAR OLD CHILDREN IN ALBERTA FIRST NATIONS COMMUNITIES, 2005-2009}

$\underline{\text { S Vik }}{ }^{1}$, R Richardson ${ }^{1}$, G Wang ${ }^{1}$, D Menon ${ }^{1}$, A Meunier ${ }^{1}$, W Yacoub ${ }^{2}$

\section{${ }^{1}$ Calgary, AB; ${ }^{2}$ Edmonton, AB}

BACKGROUND: Rates of some vaccine preventable illnesses continue to be much higher in Alberta First Nations communities compared to the general population.

PURPOSE: To report vaccination coverage among one and two year old children living on First Nations communities in Alberta between 2005 and 2009. Challenges in capturing accurate data for immunization coverage and limitations of the current data source will be discussed.

METHODS: Nurses in Alberta First Nations communities submit yearly vaccination reports to Health Canada's First Nations and Inuit Health in Alberta Region. Data include the aggregate numbers of children residing in communities by age group, and numbers receiving routine vaccinations. The proportion of children starting a routine vaccination series (uptake) and scheduled completion rates are reported for children one and two years of age.

RESULTS: From 2005 to 2009, between $73 \%$ and $93 \%$ of the 45 Alberta First Nations communities submitted vaccination coverage data each year. Among one year olds, the regional average vaccine uptake was relatively stable over this period, at $91 \%, 89 \%$ and $92 \%$ for the meningococcal C (MenC), pneumococcal (PCV7), and diphtheria, tetanus, pertussis, polio (DTaP-IPV) vaccines, respectively. The uptake among two year olds was $83 \%, 77 \%$ and $76 \%$ for the DTaPIPV, measles, mumps, rubella (MMR) and varicella zoster (VZV) respectively. Community-specific coverage rates varied considerably, with approximately $95 \%$ and $85 \%$ of communities reporting coverage rates below target levels ( $>95 \%$ coverage), for one and two year olds, respectively.

CONCLUSION: These data indicate that completion of vaccination coverage remains suboptimal in many communities. However, data for immunizations that may have been done at off-reserve health centres were not captured, and thus these coverage rates may be underestimated. Initiatives to improve continuity and tracking of health services on and off reserve are needed in order to provide a more accurate assessment of immunization coverage and other health indicators for First Nations. 
O04

EPIDEMIOLOGY OF VARICELLA INFECTION AMONG THE FOREIGN BORN IN QUEBEC

K Okrainec, B Vissandjee, M DesMeules, C Holcroft, G Bartlett, C Greenaway

Montreal, QC

BACKGROUND: Immigrants from tropical countries are more likely to be susceptible to varicella ( $>30 \%$ ) as compared to those born in Canada ( $<5 \%$ at age 20 years). Adults are more likely to develop severe varicella as compared to children. Immigrants are not routinely screened for varicella immunity nor given catch-up varicella vaccination after arrival in Canada.

PURPOSE: To describe the epidemiology of varicella among immigrants after arrival in Canada as compared to the Canadian born population.

METHODS: A retrospective population based cohort of all immigrants who arrived in Quebec from 1996-2000 and an age and sex matched Canadian born control was used. Records from the Citizenship and Immigration Canada Landed Immigrant Database were linked ( $85 \%$ successful) with the physician claims database of the Regie de l'Assurance Maladie du Quebec (RAMQ). New cases of varicella were identified by diagnostic codes (ICD-9) in the RAMQ database. Rates of new cases of varicella and rate ratios with $95 \% \mathrm{CI}$ (using Poisson distribution) were calculated in different age groups for immigrants versus controls.

RESULTS: A total of 95,645 immigrants and 95,645 Canadian controls were included. The mean age was $25.8 \pm 15$ years and $51 \%$ were female. For each age group (in years), rates of varicella for immigrants $/ 100,000$ person years and rates ratios for immigrants versus controls were: $<1$ yr $(3,240 ; 1.58), 1-4(4,726 ; 1.24), 5-9(3,317$; $1.98), 10-14(1,035 ; 3.67), 15-19(607 ; 3.14), 20-24(397 ; 2.49), 25-29$ (225; 1.56), 30-34 (232; 1.51); 35-39 (106; 1.00), 40-49 (69; 2.63), and $\geq 50(52 ; 4.98)$. All rate ratios were statistically significant $(\mathrm{p}<0.5)$ except for those aged 35-39 years.

CONCLUSION: Immigrants have a higher risk for varicella as compared to those born in Canada, with adolescents and young childbearing adults at particularly increased risk. The immigrant population would benefit greatly from targeted varicella vaccination.

\section{O05 \\ IMMUNOGENICITY AND SAFETY OF TDAP 5 (ADACEL $®$ ) ADMINISTERED 10 YEARS AFTER A PREVIOUS DOSE OF TDAP $_{5}$ OR TDAP $_{5}$-IPV (ADACEL $®$-POLIO) VACCINE IN CANADIAN ADULTS}

SA Halperin $^{1}$, E Jordanov ${ }^{2}$, D Johnson ${ }^{3}$, D Reynolds ${ }^{4}$

${ }^{1}$ Halifax, NS; ${ }^{2}$ Swiftwater, PA; ${ }^{3}$ Lyon, France; ${ }^{4}$ Toronto, ON

BACKGROUND: Tdap 5 (ADACEL, sanofi pasteur) was initially licensed in Canada in 1999 for use in persons 12-54 years of age (currently 4-64 years). Adolescents and adults immunized with Tdap5 in the early 2000s will become eligible for a 10-year adult booster of Td or, alternatively, Tdap 5 should booster doses be recommended.

PURPOSE: To assess the immunogenicity and safety of Tdap 5 readministration 10 years after a previous dose of Tdap 5 or Tdap 5 containing vaccine.

METHODS: Phase IV, open-label, multi-center comparative study conducted in Canada. Group 1 comprised adults who had received Tdap 5 or Tdap5-IPV (Adacel-Polio, sanofi pasteur) vaccines 10 years prior. Group 2 included age-balanced Tdap-naïve participants with at least 10 years elapsed time after a previous tetanus, diphtheria and/or pertussis dose. All participants were administered Tdap 5 ; immunogenicity and safety data were compared. The primary endpoints were seroprotection rates (tetanus/diphtheria) and antibody concentrations (pertussis) 1 month post-vaccination.

RESULTS: 769 participants were enrolled (Group 1=362, Group 2=407).

Post-vaccination anti-diphtheria and anti-tetanus seroprotective levels for
Group 1 (100\%, 98.5\%) were non-inferior to Group 2 (99.7\%, 96.1\%); geometric mean concentrations (GMCs) and booster response rates were similar. Tdap 5 vaccine induced robust anti-pertussis antibody responses. Post-vaccination anti-PT, anti-FHA and anti-PRN GMCs for Group $1(116,214$, and $266 \mathrm{EU} / \mathrm{mL}$, respectively) were non-inferior to Group 2 (89.2, 249 and $216 \mathrm{EU} / \mathrm{mL})$. Although non-inferiority criteria were not met for anti-FIM responses (779 vs. $1015 \mathrm{EU} / \mathrm{mL}$ ), the lower limit of the $95 \% \mathrm{CI}$ was 0.66 , close to the pre-determined threshold (>0.67). Anti-pertussis booster response rates were high in both groups $(>84 \%)$. The safety profiles were similar.

CONCLUSION: Tdap 5 vaccine is well-tolerated and immunogenic when administered as a 10-year booster to adults previously vaccinated with $\mathrm{Tdap}_{5} / \mathrm{Tdap}_{5}$-IPV vaccines compared to Tdap-naïve persons.

\section{O06}

\section{ASSESSMENT OF VACCINE EFFECTIVENESS AND HERD IMMUNITY IN A MUMPS OUTBREAK INVESTIGATION IN ONTARIO}

GH Lim ${ }^{1}$, SL Deeks ${ }^{1}$ M Simpson¹, L Gagné ${ }^{2}$, J Gubbay¹, E Kristjanson' ${ }^{1}$, C Fung1, T Mazzulli' ${ }^{1}$, N Crowcroft ${ }^{1}$

${ }^{1}$ Toronto, ON; ${ }^{2}$ North Bay, ON

BACKGROUND: An outbreak of mumps occurred in Ontario beginning in September 2009. Rapid assessment of vaccine effectiveness (VE) is an important component of outbreak management. Knowledge of VE can also be used to determine the level of coverage required to achieve herd immunity and interrupt community transmission (i.e., herd immunity threshold).

PURPOSE: The objective of this investigation was to assess VE of one and two doses of the MMR vaccine and determine the herd immunity threshold.

METHODS: Confirmed case information was retrieved from the integrated Public Health Information System in Ontario. Cases occurring between September 1, 2009 and June 10, 2010 were included. Selected health units supplied coverage data from the Ontario Immunization Record Information System. VE was calculated by dose using the screening method. Basic reproductive values (Ro) between 4 and 10 were considered for varying levels of VE.

RESULTS: 134 confirmed cases were identified. Information on receipt of mumps vaccine was available for $84 \%$. VE of one and two dose MMR ranged from $49 \%$ to $88 \%$ and $66 \%$ to $88 \%$, respectively. As VE decreased, higher coverage was required to achieve herd immunity threshold for a given Ro. Similarly, higher coverage was required as Ro increased (see table).

\begin{tabular}{|c|c|c|c|c|c|c|c|}
\hline \multirow{4}{*}{$\begin{array}{l}\text { Reproductive } \\
\text { Number }\end{array}$} & \multirow{4}{*}{$\begin{array}{l}1 / \\
\text { Reproductive } \\
\text { Number }\end{array}$} & \multirow{3}{*}{$\begin{array}{l}\text { Immunity in } \\
\text { Population } \\
\text { Required to Stop } \\
\text { Transmission }\end{array}$} & \multirow{3}{*}{\multicolumn{5}{|c|}{$\begin{array}{l}\text { Coverage Required to Achieve Herd Immunity for } \\
\text { Varying Levels of Vaccine Effectiveness }\end{array}$}} \\
\hline & & & & & & & \\
\hline & & & & & & & \\
\hline & & & $V E=$ & $V E=$ & VE= & $V E=$ & VE= \\
\hline Ro & 1/Ro & (1-1/Ro)x100\% & $75 \%$ & $80 \%$ & $85 \%$ & $90 \%$ & $95 \%$ \\
\hline 4 & 0.25 & $75.0 \%$ & $100.0 \%$ & $93.8 \%$ & $88.2 \%$ & $83.3 \%$ & $78.9 \%$ \\
\hline 6 & 0.17 & $83.3 \%$ & - & - & $98.0 \%$ & $92.6 \%$ & $87.7 \%$ \\
\hline 8 & 0.13 & $87.5 \%$ & - & - & - & $97.2 \%$ & $92.1 \%$ \\
\hline 10 & 0.10 & $90.0 \%$ & - & - & - & - & $94.7 \%$ \\
\hline
\end{tabular}

CONCLUSION: High levels of coverage are required to interrupt transmission of mumps in the community. It is essential not to become complacent about vaccination programs. 


\section{O07}

EMERGENCE OF PNEUMOCOCCAL SEROTYPE 19A IN

CANADIAN CHILDREN: IMPACT 2000-2009

JA Bettinger ${ }^{1}$, DW Scheifele ${ }^{1}$, JD Kellner ${ }^{2}$, W Vaudry ${ }^{3}$,

SA Halperin ${ }^{4}$, B Law ${ }^{5}$, G Tyrrell ${ }^{3}$; C Monitoring Program Active ${ }^{5}$

${ }^{1}$ Vancouver, $\mathrm{BC} ;{ }^{2}$ Calgary, $\mathrm{AB} ;{ }^{3}$ Edmonton, $\mathrm{AB} ;{ }^{4} \mathrm{Halifax}, \mathrm{NS}$;

${ }^{5}$ Ottawa, ON

BACKGROUND: Vaccination with the 7-valent pneumococcal conjugate vaccine (PCV7) has eliminated over $80 \%$ of invasive pneumococcal infections in Canadian children. Using the 10-valent and 13 -valent could further reduce invasive disease. This report examines the non-PCV7 serotypes and resistance patterns in Canadian children from 2000-2009.

METHODS: Active surveillance was conducted at the 12 children's hospitals of the IMPACT network from 2000 to 2009. Data for 2009 are preliminary. Eligible cases had pneumococci isolated from a normally sterile body site, mainly blood and spinal fluid. Case-finding involved both laboratory monitoring and reviews of discharge diagnosis codes. Local and referred cases were included, as were inpatient and outpatient cases. Isolates were serotyped at the National Centre for Streptococcus, Edmonton.

RESULTS: In 2008 and 2009 serotype 19A was responsible for $42 \%$ $(67 / 158)$ of invasive infections in children $<5$ years of age. The remaining invasive cases were caused by 31 serotypes with serotypes $7 F(n=11,7 \%)$ and $3(n=10,6 \%)$ accounting for the most cases after 19A. 19A cases remained steady in BC at 1-2 per year before and after implementation of PCV7 programs while increasing in Quebec and Ontario both before and after PCV7 program implementation.

The majority $(61 \%, 24 / 39)$ of $19 \mathrm{~A}$ isolates were penicillin susceptible from $2000-2004$ and penicillin resistant $(61 \%, 74 / 121)$ thereafter. From 2005-2009, 45\% (33/74), 20\% (15/74) and 5\% (4/74) of 19A penicillin resistant isolates occurred in Quebec, Ontario and BC, respectively. From 2005-2009, 11\% (13/121) of 19A isolates have been multidrug resistant, with $46 \%(6 / 13)$ of the multidrug resistant isolates over this time period occurring in AB in 2008.

CONCLUSION: Serotype 19A predominated invasive pneumococcal infections post-PCV7, but this varied regionally. While PCV7 may have contributed to the increase in 19A cases, secular trends, such as seasonal variation and antibiotic prescribing practices, appear to play a large role.

\section{O08 \\ ASSESSING THE IMPACT OF CONFOUNDING (MEASURED AND UNMEASURED) IN A TEST-NEGATIVE CASE CONTROL STUDY TO EXAMINE THE RISK OF PANDEMIC H1N1 ASSOCIATED WITH RECEIPT OF THE 2008-9 SEASONAL INFLUENZA VACCINE}

\section{LC Rosella, NS Crowcroft}

\section{Toronto, ON}

BACKGROUND: During the spring/summer of 2009, four Canadian studies reported an increased risk of pH1N1 associated with the 2008-9 seasonal influenza vaccine. Given the observational nature of the study designs, confounding may play a role.

OBJECTIVE: The primary objective of this study is to describe the impact of measured and unmeasured confounding on a test-negative case control study.

METHODS: Data were collected using a standardized telephone questionnaire. Odds ratios (OR) and 95\% confidence intervals were derived using logistic regression. The impact of measured confounders was measured using stratification, model adjustment, and propensity matching. The effect of unmeasured confounding was measured using sensitivity analysis.

RESULTS: Interviews were completed among 1640 cases and controls. Overall, pH1N1 cases were more likely to have received the seasonal influenza vaccine once adjusted for age, sex, underlying medical condition, previous primary care visits, children in the household, and travel to Mexico (OR $=1.7995 \%$ C.I. $1.23-2.60)$ and was similar when adding data from wave $2(\mathrm{OR}=1.8295 \%$ C.I. $1.24-2.66)$. The effect increased when testing was within 7 days of symptom onset: $(\mathrm{OR}=$ $2.1495 \%$ C.I. 1.23 - 3.72). The positive effect remained after propensity matching $(\mathrm{OR}=1.5695 \%$ C.I $1.12-2.16)$ and pneumococcal vaccine was not associated with $\mathrm{pH} 1 \mathrm{~N} 1$ in the adjusted model (OR $=0.8295 \%$ C.I. $0.52-1.29)$. In the sensitivity analysis, for an unmeasured confounder to result in a non-significant association, it must be strongly positively associated with $\mathrm{pH} 1 \mathrm{~N} 1(\mathrm{OR} \geq 3.0)$ and vaccine receipt $(\mathrm{OR}$ $\geq 3.5)$.

CONCLUSION: A positive association between 2008-9 seasonal influenza vaccine and pH1N1 illness was observed after adjustment for multiple confounders using different methods. That observed association is not likely explained by an unmeasured confounder given the high prevalence and strength of association needed and given that all known confounders were already included in the analysis.

\section{O09 \\ ROTAVIRUS IMMUNIZATION: KNOWLEDGE, ATTITUDES, BELIEFS AND PRACTICES OF BC PHYSICIANS}

\section{J Chan $^{1}$, V Sahni ${ }^{1}$, T Donovan $^{2}, \underline{M ~ N a u s}^{1}$ \\ ${ }^{1}$ Vancouver, BC; ${ }^{2}$ New Westminster, BC}

BACKGROUND: Rotavirus is the most common cause of gastroenteritis in children under 2 years in Canada. In 2006, a new vaccine was approved for use in Canada. In 2008, the National Advisory Committee on Immunization recommended its use for infants.

PURPOSE: To assess physician knowledge, attitudes, beliefs and practices about rotavirus infection and immunization in British Columbia (BC).

METHODS: A survey was mailed to a random sample of 1343 general practitioners (GPs) and all 257 pediatricians in BC. Physician knowledge was assessed using true/false/not sure scale, and attitudes/beliefs measured using a 4-point Likert scale. Descriptive analyses were conducted and stratified by specialty.

RESULTS: The response rate was 39\% (37\% GPs; 47\% pediatricians). Compared to GPs, pediatricians were more likely to be affiliated with a university ( $82 \%$ vs. $39 \%$ ) and spent fewer hours per week in outpatient care. Pediatricians were more knowledgeable about the vaccine; $91 \%$ (vs. 37\%) knew that intussusception was associated with a previous vaccine and $36 \%$ (vs. 15\%) knew that rotavirus vaccine should not be started in infants older than 15 weeks. Insufficient information partly contributed to knowledge gaps; $63 \%$ of GPs and 39\% of pediatricians believed that they had not received sufficient information on rotavirus vaccine. A quarter of GPs vs. $41 \%$ of pediatricians reported recommending the vaccine. Most respondents (92\%) believed routine administration would reduce severe gastroenteritis, $77 \%$ believed it should be publicly funded and $94 \%$ indicated they would recommend the vaccine if it was. Common perceived barriers to rotavirus vaccine uptake were parental reluctance to pay (97\%), parental concern about vaccine safety $(90 \%)$, and parental belief that rotavirus infection is not severe $(89 \%)$.

CONCLUSION: Support for publicly funded rotavirus vaccine among GPs and pediatricians is high. A knowledge gap for rotavirus vaccine was identified suggesting value of an education program, particularly targeting GPs. 
O10

\section{THE EPIDEMIOLOGY OF SEROGROUP B MENINGOCOCCAL INVASIVE INFECTIONS IN CANADA, IMPACT 2002-2009}

\author{
JA Bettinger ${ }^{1}$, N Le Saux ${ }^{2}$, DW Scheifele ${ }^{1}$, W Vaudry ${ }^{3}$, \\ SA Halperin ${ }^{4}$, R Bortolussi ${ }^{4}$, R Tsang ${ }^{5}$ \\ ${ }^{1}$ Vancouver, BC; ${ }^{2}$ Ottawa, ON; ${ }^{3}$ Edmonton, AB; ${ }^{4} \mathrm{Halifax}, \mathrm{NS}$; \\ ${ }^{5}$ Winnipeg, MB
}

BACKGROUND: Serogroup B meningococcal infections remain the only meningococcal serogroup that is not vaccine preventable. The epidemiology of group B infections varies by age and province. Monitoring the epidemiology of serogroup B invasive meningococcal disease (IMD) is important post-conjugate vaccine implementation.

METHODS: Active population-based surveillance was conducted across Canada by the 12 centers of the Immunization Monitoring Program, Active (IMPACT) for hospital admissions in all ages related to Neisseria meningitidis from January 2002 - December 2009. Case definition required the isolation of meningococcus or positive PCR test from a sterile site. All incidence rates are reported per 100,000.

RESULTS: A total of 665 cases were reported; 357 in children $(<20$ years) and 308 in adults. Serogroup B represented 352/665 (53\%) cases. The overall average incidence of serogroup B disease remained steady at 0.23 while serogroup $C$ incidence declined from 0.23 in 2002 to 0.08 in 2008 . The incidence of serogroup $B$ was higher in children at 0.57 than adults (0.13). From 2006-2009 the incidence of serogroup B was significantly higher than serogroup $C$ in children (0.69 vs. 0.05$)$, but not in adults $(0.17$ vs. 0.10$)$.

Serogroup B distribution varied significantly by province $(\mathrm{p}<0.001)$. Cases from Quebec represented 47\% (166/352) of the serogroup B cases nationally. Serogroup B accounted for 71\% (166/224) and 74\% $(12 / 17)$ of IMD cases in Quebec and Newfoundland compared to $41 \%$ (174/424) in other provinces.

The most common manifestation with serogroup $B$ and $C$ was meningitis at $67 \%(236 / 352)$ and $52 \%(76 / 145)$ respectively. Death occurred in 20 (3\%) serogroup B cases (10 children and 10 adults) and 19 (2.9\%) serogroup $\mathrm{C}$ cases ( 3 children and 16 adults). Sequelae occurred in 65 (9.7\%) serogroup B and 33 (4.9\%) serogroup C cases.

CONCLUSION: Due to the reduction in serogroup C IMD, serogroup B now causes the majority of IMD in Canada. The distribution of group B differs by age and province. Manifestations, morbidity and mortality are similar to group C.

\section{O11 \\ REDUCING THE PAIN OF CHILDHOOD IMMUNIZATION - AN EVIDENCE-BASED CLINICAL PRACTICE GUIDELINE A Taddio $^{1}$, M Appleton ${ }^{2}$, B Bortolussi ${ }^{2}$, C Chambers ${ }^{2}$, V Dubey ${ }^{1}$, S Halperin ${ }^{2}$, A Hanrahan ${ }^{3}$, M Ipp ${ }^{1}$, D Lockett ${ }^{1}$, N MacDonald ${ }^{2}$, D Midmer ${ }^{1}$, P Mousmanis ${ }^{1}, V_{\text {Palda }}{ }^{1}$, K Pielak ${ }^{4}$, R Pillai Riddell ${ }^{1}$, M Rieder ${ }^{5}$, J Scott ${ }^{2}$, V Shah ${ }^{1}$ ${ }^{1}$ Toronto, ON; ${ }^{2}$ Halifax, NS; ${ }^{3}$ Edmonton, AB; ${ }^{4}$ Vancouver, BC; ${ }^{5}$ London, ON}

BACKGROUND: Immunization injections are the most common source of iatrogenic pain in childhood. Pain from vaccine injections is a source of distress for children, parents and vaccinators, and, if not addressed, can lead to pre-procedural anxiety at future procedures, medical fears, and healthcare avoidance behaviours, including nonadherence with immunization schedules.

OBJECTIVE: To develop the first evidence-based guideline aimed at reducing pain during vaccine injections in children.

METHODS: Separate systematic reviews for pharmacological, physical, and psychological pain-relieving strategies were performed for the purposes of this guideline and formed the evidence. The guideline development process was based on the U.S. Task Force on Preventive Health Care. Accompanying level of evidence and grade of recommendation were made for 18 clinical questions based on the systematic reviews interpreted by an expert panel.

RESULTS: There is good evidence to support use of breastfeeding, sweet tasting solutions, injecting the least painful vaccine brand, and topical anesthetics, and fair evidence for use of specific injection techniques (rapid intramuscular injection without aspiration, and injecting the most painful vaccine last if multiple injections are given) and psychological techniques (clinician-led or child-led distraction, slow deep breathing, combined cognitive-behavioural interventions). There is fair evidence to offer parent interventions (parent-led distraction, parent coaching) or tactile stimulation. There is good evidence to recommend against positioning children supine and suggesting to children that vaccine injections 'do not hurt'. There is insufficient evidence or no evidence to recommend skin-cooling techniques (vapocoolants, ice/cold packs), simultaneous vaccine injections, a specific route of administration (intramuscular, subcutaneous), or oral analgesics.

CONCLUSIONS: Managing pain uses a "3-P" approach: Pharmacological, Physical and Psychological strategies. In selecting specific strategies to use, clinicians and parents are advised to consider the effectiveness of individual modalities, the goals of therapy, and the preferences of children, parents and clinicians. Combining strategies may improve pain relief.

\section{2}

\section{THE CANADIAN CENTER FOR VACCINOLOGY HUMAN VACCINE CHALLENGE UNIT: EXPANDING MEDICAL FRONTIERS}

\section{SA McNeil, M Kiberd, S Halperin}

Halifax, NS

INTRODUCTION: In 2009, the Canadian Center for Vaccinology (CCfV) opened the first Canadian microbial challenge unit. Human challenge studies offer potential to accelerate vaccine development by enabling establishment of proof-of-concept of vaccine effectiveness (VE) with small numbers of participants. While classical VE studies rely upon natural exposure to infection and therefore require large sample sizes, deliberate exposure of vaccinated subjects to the pathogen against which they were immunized allows these studies to be done with small numbers of participants. In the case of evaluation of vaccines to which natural exposure in the environment does not occur, such as agents of bioterrorism or novel respiratory pathogens, challenge studies offer the only viable means of estimating VE. Challenge studies also offer novel means to study microbe-host interactions, transmissibility, and natural history of human infections.

METHODS: The CCfV Challenge Unit is a 10-bed inpatient clinical research unit, which can accommodate multiple experimental designs. Capacity for strict isolation of participants in state-of-the-art fully equipped negative pressure rooms will allow the evaluation of new vaccines against respiratory pathogens or other pathogens with potential for human-to-human transmission.

DISCUSSION: The CCfV Vaccine Challenge Unit offers exciting, unique Canadian infrastructure that will allow researchers in Canada and around the world, in the private sector, in governments, and in academic institutions, to more efficiently test the effectiveness of early stage vaccines, potentially drastically shortening the time for vaccine development leading to earlier availability of new vaccines for the protection of Canadians. However, given the paucity of such units in the world, the increasingly complex regulatory environment in North America and lack of case law or specific guidelines to govern the conduct of human challenge studies in Canada, CCfV bears the responsibility to ensure that studies conducted in the Unit are carefully selected after rigorous scientific and ethical review and are subject to diligent oversight. The legal and ethical framework upon which to judge the quality and safety of human challenge studies in the Canadian context will be explored. 
013

\section{EFFICACY OF INFLUENZA VACCINE FOR PREVENTING SYMPTOMATIC ILLNESS GIVEN INFECTION: RESULTS FROM A CLUSTER RANDOMIZED TRIAL IN HUTTERITE COLONIES}

$\underline{\text { M Loeb }}{ }^{1}$, M Russell ${ }^{2}$, L Moss ${ }^{1}$, K Fonseca ${ }^{2}$, D Earn ${ }^{1}$, F Aoki ${ }^{3}$, G Horsman $^{4}$, P Van Caeseele ${ }^{3}$, K Chokani ${ }^{5}$, M Vooght ${ }^{6}$, L Babiuk $^{7}$, R Webby ${ }^{8}$, S Walter ${ }^{1}$

${ }^{1}$ Hamilton, ON; ${ }^{2}$ Calgary, AB; ${ }^{3}$ Winnepeg, MB; ${ }^{4}$ Regina, SK; ${ }^{5}$ Swift Current, SK; ${ }^{6}$ Moose Jaw, SK; ${ }^{7}$ Edmonton, AB; ${ }^{8}$ Memphis, TN BACKGROUND: Influenza vaccine clinical trials usually present vaccine efficacy for infection-confirmed symptomatic illness. Vaccine efficacy for illness given infection (VEP) estimates the degree to which the vaccine prevents an infected individual from developing symptomatic illness. There are sparse data on estimates of indirect benefit of influenza vaccine on VEP.

PURPOSE: To estimate VEP of entire communities when vaccinating children and adolescents with inactivated influenza vaccine.

METHODS: Data from the 2008-2009 season of a cluster randomized trial involving 947 Canadian children and adolescents aged 36 months to 15 years who received study vaccine and 2326 community members who did not receive the study vaccine in 49 Hutterite colonies in Alberta, Saskatchewan, and Manitoba in 2008-2009. Children were randomly assigned according to community and in a blinded manner to receive standard dosing of either inactivated trivalent influenza vaccine or hepatitis A vaccine, which was used as a control. Outcomes were symptomatic illness given RT-PCR confirmed influenza A and B or a four-fold rise in serum hemagglutination inhibition (HI) titers to A/Brisbane/H1N1, A/Brisbane/H3N2, or B/Brisbane.

RESULTS: The mean rate of study vaccine coverage among eligible participants was $83 \%$ (range, 53\%-100\%) for the influenza vaccine colonies and 79\% (range, 50\%-100\%) for the hepatitis A vaccine colonies. 912 of $3272(27.9 \%)$ of all participants had either influenza A or B detected by PCR or a four-fold rise in HI titers to influenza A or B. $162 / 260(61.6 \%)$ in the influenza vaccine colonies and $371 / 490$ $(75.7 \%)$ in the hepatitis A vaccine colonies had symptomatic illness. When adjusted for the effect of clustering and receipt of non-study vaccine, VEP was $49 \%$ ( $9 \%$ to $71 \%$ ), $\mathrm{P}=0.02$. When considering the indirect effect alone (excluding vaccinated children), VEP was $60 \%$ ( $4 \%$ to $84 \%), P=0.04$.

CONCLUSION: Immunizing children and adolescents with inactivated influenza vaccine led to a significant vaccine efficacy for preventing illness given infection (VEP) in Hutterite communities.

\section{O14}

IMPACT NETWORK ACTIVE SURVEILLANCE FOR ADVERSE EVENTS FOLLOWING ADJUVANTED PANDEMIC INFLUENZA VACCINE IN CANADIAN CHILDREN LJ Sauve ${ }^{1}$, J Bettinger ${ }^{1}$, W Vaudry ${ }^{2}$, D Moore ${ }^{3}$, D Scheifele', S Halperin ${ }^{4}$, B Law ${ }^{5}$; T Canadian Immunization Monitoring Program, Active (IMPACT) ${ }^{5}$

${ }^{1}$ Vancouver, BC; ${ }^{2}$ Edmonton, AB; ${ }^{3}$ Montreal, QC; ${ }^{4}$ Halifax, NS; ${ }^{5} \mathrm{Ottawa}, \mathrm{ON}$

BACKGROUND: During the 2009 influenza (pH1N1) pandemic, Canada used an adjuvanted H1N1 monovalent influenza vaccine and limited quantities of unadjuvanted vaccine for mass vaccination. Active surveillance was one of several methods of monitoring for adverse events following immunization (AEFIs).

METHODS: The Immunization Monitoring Program, Active (IMPACT) has performed active surveillance for possible AEFIs leading to hospitalization in Canadian children's hospitals since 1991, expanding to 12 sites by 1999 . Case definitions included seizures or other unusual events within 72 hours, neurological disorders within 42 days, or thrombocytopenia within 30 days of vaccination. Preliminary data are presented here.
RESULTS: Forty-nine children were admitted to IMPACT hospitals with suspected AEFIs after $\mathrm{pH} 1 \mathrm{~N} 1$ vaccination between October 2009 and January 2010. The median age was 2.3 years (range 6 months to 18 years). AEFIs included seizures (17), other neurologic events (12), fever (11), thrombocytopenia (4) and other symptoms (5). Of the children with neurologic events, 3 had Guillain Barré syndrome. One had neurological symptoms after both doses, with an ultimate diagnosis of acute disseminated encephalomyelitis. Other presentations included cerebellitis, cerebritis, transverse myelitis, and encephalitis. Of 11 children with fever, 6 had symptoms of viral infections and 3 had Kawasaki disease. The length of hospitalization ranged from $1-51$ days. There were no deaths, and no hospitalizations due to anaphylaxis. Seventeen (35\%) children had likely alternative causes for their symptoms. During October to January in previous years, an average of 4.3 admissions for post-immunization neurologic events and 12.6 for post-immunization seizures were reported by IMPACT.

CONCLUSIONS: A serious AEFI rate of approximately 1 per 100,000 doses of $\mathrm{pH} 1 \mathrm{~N} 1$ vaccine distributed was reported by the Public Health Agency of Canada, a rate not different from that expected with seasonal influenza vaccine. IMPACT surveillance identified serious AEFIs in 49 children, with a 3-fold increase in numbers of neurological events compared with previous seasons. Evaluation of causality is pending.

\section{O15}

PCIRN STUDY OF IMMUNOGENICITY OF MONOVALENT, ADJUVANTED H1N1 VACCINE IN ABORIGINAL ADULTS E Rubinstein $^{1,2}$, DW Scheifele ${ }^{3}$, G Hammond ${ }^{1,2}$, F Aoki, ${ }^{1,2}$, G Predy 2,4, C Sikora ${ }^{2,4}$, L Sauve ${ }^{2,3}$, Y Li ${ }^{1,2}$, B Law ${ }^{3,5}$, S Halperin 2,5, B Smith ${ }^{2}$

${ }^{1}$ Winnipeg, MB; ${ }^{2}$ Halifax, NS; ${ }^{3}$ Vancouver, BC; ${ }^{4}$ Edmonton, AB; ${ }^{5}$ Ottawa, ON

BACKGROUND: When pandemic H1N1 2009 vaccine was recommended for all Canadian adults, it was not known if the standard adult dose of adjuvanted vaccine would elicit satisfactory antibody responses in First Nations and Metis people, some of whom were severely affected during the first pandemic wave. Responsiveness of aboriginal Canadians to influenza vaccination has not been systematically studied.

PURPOSE: To assess the immunogenicity of pH1N1 adjuvanted vaccine in aboriginal adults.

METHODS: Three PCIRN centers enrolled aboriginal adults 20 59 years of age to receive one dose $(0.5 \mathrm{~mL})$ of monovalent, adjuvanted H1N1 2009 vaccine (Arepanrix ${ }^{\circledR}$, GSK Laval) from a single lot. Those with immune compromise were excluded. Blood samples were obtained at study entry and 21 days after vaccination. Serum antibody titers were measured by hemagglutination inhibition assay (HAI) and evaluated using the standard EMEA/CHMP criteria for adults $<60$ years of age.

RESULTS: In total 138 subjects participated (First Nations 95, Metis 42, Inuit 1 ); $66 \%$ were female. Mean age was 37.3 years (range 20 57 years). Significant health conditions were reported by 105 subjects $(76 \%)$. Antibody to $\mathrm{pH} 1 \mathrm{N1}$ was detectable (titer $\geq 10$ ) in 47 subjects $(34 \%)$ at baseline, with titers $\geq 40$ in $23(17 \%)$. After vaccination 135 of 136 subjects tested (99.3\%) had a titer $\geq 40$ ("protective"), including $88 / 89$ (98.9\%) of those with no pre-existing antibody (unprimed). The geometric mean titer (GMT) following vaccination was 437 in the whole group, 361 in unprimed subjects and 626 in subjects with detectable pre-existing antibodies. A 4-fold antibody rise was evident in $94 \%$ of subjects, including $99 \%$ of unprimed subjects. The geometric mean fold rise in titers was 45 (95\% CI 35.1-58.1) for the whole group. No serious adverse events were encountered.

CONCLUSIONS: The recommended dose of adjuvanted pH1N1 vaccine elicited robust antibody responses in this group of aboriginal adults by all criteria. Response rates and GMT were higher than in non-aboriginal adults in a concurrent PCIRN study using the same design and lot of vaccine. 
016

ONE DOSE OR TWO? RESULTS OF A PCIRN STUDY OF DOSING REQUIREMENTS OF TODDLERS GIVEN ADJUVANTED H1N1 INFLUENZA VACCINE DURING THE 2009 PANDEMIC

DW Scheifele ${ }^{1,2}$, B Ward ${ }^{3,4}$, M Dionne ${ }^{2,4}$, G De Serres ${ }^{2,4}$, J Langley ${ }^{4}$, S Halperin ${ }^{4}$, O Vanderkooi ${ }^{4,5}$, J Kellner ${ }^{5}$,

S Dobson ${ }^{1,4}$, L Sauve ${ }^{1,4}$, Y Li, 4,6, B Law ${ }^{4,7}$

${ }^{1}$ Vancouver, BC; ${ }^{2}$ Quebec City, QC; ${ }^{3}$ Montreal, QC; ${ }^{4}$ Halifax, NS; ${ }^{5}$ Calgary, AB; ${ }^{6}$ Winnipeg, MB; ${ }^{7}$ Ottawa, ON

BACKGROUND: When adjuvanted $\mathrm{pH} 1 \mathrm{~N} 1$ vaccine was recommended for Canadian toddlers, their dosing requirement was uncertain. Two doses were recommended but early reports from Spain suggested that one dose was sufficient and a second dose caused fever more often.

PURPOSE: To compare responses of toddlers to one and two doses of $\mathrm{H} 1 \mathrm{~N} 1$ vaccine.

METHODS: Five PCIRN centers enrolled children 6-35 months old to receive 2 pediatric doses $(0.25 \mathrm{~mL})$ of adjuvanted $\mathrm{pH} 1 \mathrm{~N} 1$ vaccine (Arepanrix ${ }^{\circledR}$, GSK Laval, single lot) 21 days apart. HAI antibody to pH1N1 was measured in serum at study entry and 21 days after each dose. Safety observations were recorded daily by parents for one week after each dose and assembled adverse event rates compared by dose.

RESULTS: 167 children (mean age 20 months) had dose one, 152 had dose two and 148 completed follow-up. At study entry, 31 (18.6\%) were seropositive (HAI titer $\geq 10$ ). After dose one, 97/122 subjects (79.5\%, 95\% CI 71.3-86.3) who were seronegative at entry had a titer $\geq 40$. After dose two, all 146 subjects tested had a titer $\geq 40$. Among those seronegative at entry, the geometric mean titers (GMT) were 69.6 after dose one and 735.7 after dose two. Those seropositive at entry had a GMT of 1871.2 after dose two. Minor adverse events reflecting pain at the injection site were equally common after each dose but short-lived. Fever $\geq 39.0{ }^{\circ} \mathrm{C}$ occurred in 9 subjects $(5.4 \%)$ after dose 1 and in 10 after dose $2(6.6 \%, p=0.20)$. Medical advice was sought for 31 subjects (18.6\%) mainly for intercurrent infections.

CONCLUSIONS: One pediatric dose of adjuvanted $\mathrm{pH} 1 \mathrm{~N} 1$ vaccine elicited potentially protective titers $(\geq 40)$ in $80 \%$ of seronegative children while a second dose raised this to $100 \%$ and increased GMT by 10 -fold. Such high titers may afford greater or longer-lasting protection. Safety profiles of first and second doses did not differ; both were reasonably well tolerated.

\section{O17}

\section{ASSESSING INFLUENZA IMMUNIZATION COVERAGE IN} CHILDREN AGED 6-23 MONTHS

\section{$S$ David, $\underline{M}$ Naus}

\section{Vancouver, BC}

BACKGROUND: Influenza vaccine (TIV) has been recommended for healthy 6-23 month olds in British Columbia (BC) since 2004. The immunization registry is incomplete for this vaccine, and we used survey methods to obtain an estimate of its uptake.

PURPOSE: The primary objectives were to establish valid estimates of TIV coverage in 6-23 month olds and their household contacts during the $2007 / 8$ or $2008 / 9$ seasons. Secondary objectives were to identify missed opportunities for influenza vaccination, determine association with receipt of other routine vaccines, and assess TIV recording in the registry.

METHODS: A random sample of children aged 6-23 months during the influenza season was obtained from the BC immunization registry in which 16 of 18 health areas participate. A standardized survey was administered by telephone.

RESULTS: 1833 or $90 \%$ of eligible respondents completed the survey. Based on parent/ guardian response, $23 \%$ of infants/toddlers had received TIV in the previous season, although $58 \%$ knew that it was recommended. No adults had received TIV in $67 \%$ of the households, and no other children in $79 \%$. The following were significantly associated with TIV receipt: recommendation by the provider; knowing it was recommended or free free; and commencement of routine childhood vaccines. Half of children who had not received TIV had received other vaccines in November through March. The main reason for non-receipt of TIV was 'don't believe it's necessary'. The immunization registry contained a record for $44 \%$ of children reportedly immunized.

CONCLUSION: TIV coverage rates among infants/toddlers in BC and their household contacts were low. Despite awareness of the recommendation, many parents/guardians did not believe that influenza vaccination is important, suggesting a gap in knowledge about the rationale for this recommendation. Most children who did not receive TIV did receive other routine vaccines during the seasonal period.

\section{O18}

\section{PANDEMIC H1N1 INFLUENZA VACCINE POST-CAMPAIGN SURVEY: H1N1 IMMUNIZATION COVERAGE AND PUBLIC ATTITUDES IN BRITISH COLUMBIA (BC)}

\section{A Schneeberg, M Chong, J Bettinger, M Naus}

\section{Vancouver, BC}

BACKGROUND: Pandemic H1N1 vaccination started in $\mathrm{BC}$ on October 26th 2009. Vaccine was available through public health and private providers. Mass clinics were held until December 18th 2009. Aggregate weekly doses administered by public health were reported online to a central data base, with limited information available on doses given by physicians, for an overall estimate of $38 \%$ coverage.

PURPOSE: To provide an accurate estimate of $\mathrm{H} 1 \mathrm{~N} 1$ vaccine uptake by age and priority status and to detail the frequency and severity of self reported adverse events.

METHODS: Random digit dialing was used to administer a telephone survey across BC. Any individual who answered the call and was $19+$ years of age was asked about the uptake of H1N1 vaccine for all members of their household. Only one individual from each household was used for estimation of coverage to avoid a cluster effect.

RESULTS: A total of 4850 households were surveyed. Age adjusted reported receipt of $\mathrm{H} 1 \mathrm{~N} 1$ vaccine was $42.7 \%$. Individuals aged $65+$ reported $57.9 \%$ uptake. Those aged $10-19$ and $20-39$ years had the lowest coverage at $32.2 \%$ and $32.8 \%$ respectively. Based on self-reports from all individuals from all households, $75.9 \%$ of 352 pregnant women reported H1N1 vaccine receipt, as did $67.63 \%$ of 1900 people who reported a chronic condition and $62.6 \%$ of 872 health care workers. Of all survey respondents who received the vaccine, $39.0 \%(n=894)$ indicated they experienced 'any side effect' of which 68.0\%, 28.5\%, and $3.4 \%$ reported this as mild, moderate or severe, respectively.

CONCLUSION: The H1N1 coverage estimate found by this survey was comparable to the results found in the online reporting done throughout the campaign. Age specific coverage rates were lowest in adolescents and young adults. H1N1- risk groups reported higher uptake, indicating that messaging for this vaccine reached the desired audience. 


\section{POSTERS}

\section{CLINICAL VACCINOLOGY SCIENCE}

\section{P001}

INFLUENCE OF PRODROMAL PAIN ON SEVERITY OF ACUTE HERPES ZOSTER AND UTILIZATION OF HEALTH CARE RESOURCES

A Benbernou ${ }^{1}$, M Drolet ${ }^{1}$, M Levin ${ }^{2}$, K Schmader ${ }^{3}$, M Oxman ${ }^{4}$, R Johnson ${ }^{5}$, D Patrick ${ }^{5}$, S Camden ${ }^{1}$, J Mansi ${ }^{6}$, M Brisson ${ }^{1}$ ${ }^{1}$ Quebec, QC; ${ }^{2}$ Denver, CO; ${ }^{3}$ Durham, NC; ${ }^{4}$ La Jolla, CA; ${ }^{5}$ Vancouver, BC; ${ }^{6}$ Montreal, QC

BACKGROUND: About $30 \%$ of individuals living in developed countries will develop herpes zoster in their lifetime. Herpes zoster is the results of reactivation of the varicella zoster virus, which is often accompanied by a prodrome of dermatomal pain. Little is known about the characteristics of prodromal pain and its impact on utilization of health care resources.

PURPOSE: 1) Describe the frequency, severity and duration of prodromal pain;2) Determine the influence of prodromal pain on the severity of acute herpes zoster and on utilization of health care resources.

METHODS: Between 10/2005 and 07/2006, 251 subjects $\geq 50$ years old, seeking care for herpes zoster within 14 days of rash onset, were recruited by 83 physicians across Canada. Severity and duration of prodromal pain were measured retrospectively using the Initial Zoster Impact Questionnaire. The burden of prodromal pain was obtained by the product of pain severity and duration.

RESULTS: The majority of participants reported having had prodromal pain (74\%). Mean pain duration and severity were 4.7 days and 6/10, respectively. Subjects aged $61-70$ years old were more likely to report prodromal pain $(\mathrm{PR}=1.14, \mathrm{p}$-value $=0.02)$ than those $<60$ years old. The burden of prodromal pain was greater in subjects unemployed/retired $(\mathrm{p}$-value $=0.02)$ and immunosuppressed $(\mathrm{p}$-value $=$ 0.04). Having had prodromal pain was associated with more severe pain during the acute phase of herpes zoster (6.2 vs. 4.3 , $\mathrm{p}$-value $=$ $<.0001)$. Finally, subjects who reported having had prodromal pain were more likely to receive antivirals $(\mathrm{RR}=1.18,95 \% \mathrm{CI}, 1.00-1.40)$ and to visit the emergency room $(\mathrm{RR}=2.56,95 \% \mathrm{CI}, 1.05-6.25)$.

CONCLUSION: These data suggest that burden of prodromal pain is non-negligible and should be considered when evaluating the overall benefit of vaccinating against herpes zoster.

\section{P002}

EPIDEMIOLOGY OF ROTAVIRUS ACUTE GASTROENTERITIS IN CHILDREN IN A REGION OF QUEBEC, CANADA

\section{(2002-2008)}

S Bernard ${ }^{1}$, L Valiquette ${ }^{1}$, C Cyr $^{1}$, C Babakissa ${ }^{1}$,

T Cote-Boileau ${ }^{1}$, P De Wals ${ }^{2}$, A Gagneur ${ }^{1}$

${ }^{1}$ Sherbrooke, QC; ${ }^{2}$ Laval, QC

BACKGROUND: Available literature on rotavirus acute gastroenteritis (AGE) surveillance rarely includes data on short term hospitalization and/or outpatient visits amongst the same population.

OBJECTIVE: The purpose of this study was to assess the burden of rotavirus AGE on the whole health care system.

METHODS: A retrospective cohort study of all children $<5$ years old with an AGE from 2002-2008 was performed in Estrie, a rural region in south eastern Quebec, Canada (pop.: 298,780). Data on hospital admissions and emergency department (ED) visits came from the only hospital who serves this region, outpatient data came from public insurance system. Since identification for rotavirus was not systematic, we used two methods of indirect estimation.

RESULTS: A total of 435 to 719 rotavirus associated hospitalizations were estimated during a 6-year period. Short-term hospitalizations represented $54 \%$ of all hospitalizations. The estimated ED and ambulatory visits due to rotavirus was respectively 1009 to 1361 and 1613 to 1687 for the period. The epidemic curve showed a periodicity with higher incidence in March and April. The annual incidence rate of rotavirus AGE hospitalization was estimated between 49 to $80 / 10,000$ children), emergency department visits ranged from 113 to $152 / 10,000$ and ambulatory visits ranged from 180 to $188 / 10,000$.

CONCLUSION: Most available retrospective studies probably underestimated rotavirus hospitalizations because they did not take into account short term hospitalizations. Furthermore, our data on emergency and outpatient visits give an exhaustive appraisal of the burden of rotavirus, a crucial information to the evaluation of immunization programs.

\section{P003}

VACCINATION OF CHILDREN WITH PNEUMOCOCCAL CONJUGATE VACCINE HAD NO IMPACT ON HOSPITAL ADMISSIONS FOR PNEUMONIA IN ADULTS IN QUEBEC P De Wals ${ }^{1}$, M Carbon ${ }^{2}$, É Fortin ${ }^{1}$, J Pépin ${ }^{3}$, M Douville Fradet ${ }^{1}$ ${ }^{1}$ Quebec City, QC; ${ }^{2}$ Rennes, France; ${ }^{3}$ Sherbrooke, QC

BACKGROUND: In the province of Quebec, Canada, a publicly funded pneumococcal conjugate vaccine program for children was implemented in December 2004 and uptakes rates were high. A statistical analysis was performed to assess a possible indirect effect of this program on the frequency of hospital admissions for all-cause pneumonia in adults.

METHODS: Time-series analysis of provincial hospital database for the period 1990-2007, using structural dynamic modeling and taking into account seasonality, secular trend, level breaks, outliers, and the candidate explanatory variable: the monthly proportion of vaccinated children as measured in Quebec City.

RESULTS: A total of 211,644 admissions with a main diagnosis of pneumonia was recorded in the study population representing close to 100 million person-years of observation. The average admission rate was 44/100,000 person-years in the group 20-39 years, 90/100,000 in the group 40-59 years, $493 / 100,000$ in the group $60-79$ years and $1,813 / 100,000$ in elderly adults. Seasonality was a major factor in all age groups and a winter peak was recorded every year. High monthly values were associated with influenza epidemics. A downward break in the baseline rate was identified in adults less than 60 years of age in 1999, possibly resulting from a change in the criteria for hospitalization. There was no downward break in rates following the introduction of the pneumococcal conjugate vaccine program and the increasing proportion of vaccinated children in the population was not related to a proportional decline in the frequency of hospital admissions for pneumonia in any age category.

CONCLUSION: Although an indirect impact of the pneumococcal conjugate vaccination of children on the frequency of pneumonia caused by the pneumococcal serotypes included in the vaccine cannot be excluded, no substantial reduction in the frequency of hospital admissions for all-cause pneumonia was observed in adults following the introduction of the program.

\section{P004}

IMMUNOGENICITY AND SAFETY OF A RECOMBINANT MENINGOCOCCAL SEROGROUP B VACCINE AND A QUADRIVALENT CONJUGATE VACCINE IN LABORATORY WORKERS

\section{P Dull ${ }^{1}$, H Wang ${ }^{2}$, A Annett Kleinschmidt ${ }^{3}$, D Toneatto ${ }^{4}$,}

A Kimura ${ }^{1}$

${ }^{1}$ Cambridge, MA; ${ }^{2}$ Amsterdam, Netherlands; ${ }^{3}$ Marburg, Germany; ${ }^{4}$ Siena, Italy

BACKGROUND: Laboratory staff exposed to meningococci are at increased risk for invasive disease. As such, the National Advisory Committee on Immunisation (NACI) recommends that, research, 
industrial and clinical laboratory personnel who are routinely exposed to $N$. meningitides be immunised against meningococcal disease.

METHODS: This was the first study in laboratory workers who received both a conjugate vaccine against meningococcal serogroups A, C, W-135 and Y (Men ACWY-CRM, Menveo ${ }^{\circledR}$ ) and an investigational multicomponent vaccine against serogroup $\mathrm{B}$ containing factor $\mathrm{H}$ binding protein, Neisserial Adhesin A, Neisseria Heparin Binding Antigen and New Zealand strain outer membrane vesicles (4CMenB). Healthy adults (18-50 years of age) received 3 doses serogroup $B$ vaccine at baseline, 2 and 6 months followed by a single dose of MenACWY-CRM 1 month later. Immunogenicity was assessed via serum bactericidal assay using human complement (hSBA) 1 month postvaccination; solicited reactogenicity and adverse events were monitored.

RESULTS: Fifty-four participants enrolled. Bactericidal immune responses were evident after each dose of $4 \mathrm{CMenB}$, as assessed by geometric mean titers and percentages of subjects with hSBA titers $=4$ or $=8$ or a four-fold rise over baseline. One month postvaccination, most MenACWY-CRM recipients had hSBA titers $=8$ against serogroups A, C, W-135, and Y. Both vaccines were well-tolerated, although rates of solicited reactions were lower after MenACWY-CRM.

CONCLUSIONS: Laboratory workers who received $4 \mathrm{CMenB}$ and MenACWY-CRM showed evidence of bactericidal immune responses to all 5 serogroups. Both vaccines were well-tolerated in these participants.

\section{P005}

IMMUNOGENICITY OF AN INVESTIGATIONAL MULTICOMPONENT MENINGOCOCCAL SEROGROUP B VACCINE IN HEALTHY INFANTS AT 2, 4 AND 6 MONTHS OF AGE

T Vesikari ${ }^{1}$, S Esposito ${ }^{2}$, A Kimura ${ }^{3}$, A Kleinschmidt ${ }^{4}$, E Ypma ${ }^{5}$, D Toneatto ${ }^{6}$, P Dull $^{3}$

${ }^{1}$ Tampere, Finland; ${ }^{2}$ Milano, Italy; ${ }^{3}$ Cambridge, MA; ${ }^{4}$ Marburg, Germany; ${ }^{5}$ Amsterdam, Netherlands; ${ }^{6}$ Siena, Italy

BACKGROUND: Meningococcal serogroup B disease is the most widespread form of bacterial meningitis and septicemia in Canadian infants for which there is no licensed, broad-spectrum vaccine. Genomic technology was employed to develop a multicomponent meningococcal serogroup $B$ vaccine comprised of three recombinant protein antigens: Factor $\mathrm{H}$ binding protein (fHbp), Neisserial Adhesin A ( NadA), Neisseria Heparin Binding Antigen (NHBA), which were combined with New Zealand Strain 98/254 outer membrane vesicles. Initial data in Phase 2 studies showed immune responses in healthy infants.

METHODS: 3630 healthy infants were randomized to receive one of three lots of investigational vaccine with routine infant vaccines (a diphtheria, tetanus, acellular pertussis, inactivated poliovirus, Haemophilus influenzae type $\mathrm{b}$, and hepatitis $\mathrm{B}$ combination vaccine as well as a 7-valent pneumococcal conjugate vaccine), routine vaccines alone, or meningococcal $\mathrm{C}$ conjugate vaccine plus routine vaccines at ages 2, 4, and 6 months. Primary immunogenicity assessment was based on a serum bactericidal assay using human complement (hSBA) against three serogroup B strains (5/99, NZ98/254 and H44/76) 30 days after the final study vaccination.

RESULTS: Evidence of immune responses to the investigational vaccine, as assessed by hSBA titers, is anticipated. Consistency of immune response across the 3 lots and immune responses to the routine vaccines will be presented.

CONCLUSIONS: The immunogenicity profile of an investigational multicomponent serogroup B vaccine following 3 doses in healthy infants, in the context of routine vaccinations, will support further investigation.
P006

TOLERABILITY OF A THREE-DOSE SCHEDULE OF AN INVESTIGATIONAL, MULTICOMPONENT MENINGOCOCCAL SEROGROUP B VACCINE AND ROUTINE INFANT VACCINES IN A LOT CONSISTENCY TRIAL

S Esposito ${ }^{1}$, T Vesikari ${ }^{2}$, A Kimura ${ }^{3}$, E Ypma ${ }^{4}$, D Toneatto ${ }^{5}$, P Dull ${ }^{3}$

${ }^{1}$ Milano, Italy; ${ }^{2}$ Tampere, Finland; ${ }^{3}$ Cambridge, MA;

${ }^{4}$ Amsterdam, The Netherlands; ${ }^{5}$ Siena, Italy

BACKGROUND: After serogroup C, serogroup B has caused the second highest burden of disease within Canada. Rates are particularly high in infants and children $<4$ years of age. A multicomponent meningococcal serogroup $B$ vaccine comprised of recombinant protein antigens and outer membrane vesicles was developed via genomic technology. Infants are a key age group affected by serogroup B disease.

METHODS: Healthy infants $(n=3630)$ were randomized to receive one of three lots of the multicomponent serogroup $B$ vaccine with routine infant vaccines (a diphtheria, tetanus, acellular pertussis, inactivated poliovirus, haemophilus influenzae type $\mathrm{b}$, and hepatitis B combination vaccine as well as a 7 -valent pneumococcal conjugate vaccine), the routine vaccines alone, or monovalent $C$ conjugate vaccine plus routine vaccines at ages 2, 4, and 6 months. Solicited injection site and systemic reactions were recorded for 7 days postvaccination; adverse events were evaluated throughout the study.

RESULTS: The serogroup B vaccine was considered generally welltolerated when administered with routine immunizations; few infants discontinued the study due to reactogenicity following any study vaccination. In preliminary data, safety outcomes were generally similar for the three lots of investigational vaccine. The most commonly reported solicited reactions in all groups were sleepiness, irritability, unusual crying, as well as injection site tenderness, erythema, and induration. Updated data will be presented. The most frequently reported adverse events were otitis media, upper respiratory tract infections, bronchitis, and nasopharyngitis.

CONCLUSIONS: The multicomponent meningococcal serogroup B vaccine had an acceptable safety profile when administered with routine immunizations. Few vaccinees discontinued the study due to reactogenicity.

\section{P007}

EARLY CLINICAL DEVELOPMENT OF A NOVEL, MULTICOMPONENT MENINGOCOCCAL SEROGROUP B VACCINE (4CMENB)

P Dull ${ }^{1}$, M Pizza ${ }^{2}$, D Toneatto ${ }^{2}$, L De Tora ${ }^{1}$, E Ypma ${ }^{3}$,

A Kleinschmidt ${ }^{4}$, A Kimura ${ }^{1}$

${ }^{1}$ Cambridge, MA; ${ }^{2}$ Siena, Italy; ${ }^{3}$ Amsterdam, Netherlands;

${ }^{4}$ Marburg, Germany

BACKGROUND: After serogroup C, serogroup B has caused the second highest burden of disease within Canada. There is currently no vaccine available in Canada for serogroup B. Reverse vaccinology identified proteins included in a recombinant meningococcal serogroup $\mathrm{B}$ vaccine $(\mathrm{rMenB})$ that has been evaluated alone or as a multicomponent formulation with New Zealand Strain 98/254 outer membrane vesicles (4CMenB).

METHODS: In completed and ongoing studies, $4 \mathrm{CMenB}$ was administered to adults (phase 1), infants and toddlers (phase 2 and 3 ) in clinical trials as 3-and 4-dose schedules with $\mathrm{rMenB}$, meningococcal conjugate vaccine, and placebo controls. Immunogenicity of $4 \mathrm{CMenB}$ and $\mathrm{rMenB}$ was assessed via serum bactericidal assays using exogenous human complement (hSBA) against three serogroup $B$ indicator strains (5/99, NZ98/254 and H44/76). Solicited injection site and systemic reactions were recorded for 7 days after each vaccination. Adverse events were evaluated for 6 months after final vaccinations. 
RESULTS: Immunogenicity, assessed by hSBA titers, was observed in all study populations; rMenB was somewhat less immunogenic compared with $4 \mathrm{CMenB}$. A high percentage of vaccinees had seroresponse after 2 doses of $4 \mathrm{CMenB}$; immunogenicity outcomes were similar following 3 or 4 doses. Both rMenB and $4 \mathrm{CMenB}$ were more reactogenic at the injection site than placebo or meningococcal conjugate or polysaccharide vaccine controls. Most $4 \mathrm{CMenB}$ recipients reported injection-site pain after the first vaccination; fewer reported pain with subsequent doses or a booster. Solicited reactions were generally mild to moderate and self-limited. Updated data will be presented.

CONCLUSIONS: A multicomponent meningococcal serogroup B vaccine, $4 \mathrm{CMenB}$, had a favorable immunogenicity and tolerability profile supporting further study in phase 3 .

\section{P008}

CLINICAL IMMUNOGENICITY AND REACTOGENICITY OF MENVEO ${ }^{\circledR}$ (MENACWY-CRM) VERSUS MENACTRA ${ }^{\circledR}$ (MCV4) IN HEALTHY 2-TO-10-YEAR-OLD CHILDREN

SA Halperin ${ }^{1}$, A Gupta ${ }^{2}$, R Jeanfreau ${ }^{3}$, NP Klein ${ }^{4}$, K Reisinger ${ }^{5}$,

E Walter ${ }^{6}$, L Bedell7 ${ }^{7}$ M McCarthy ${ }^{7}$, A Karsten ${ }^{7}$, CJ Gill7, P Dull ${ }^{7}$

${ }^{1}$ Halifax, NS; ${ }^{2}$ Etobicoke, ON; ${ }^{3}$ Metairie, LA; ${ }^{4}$ Oakland, CA;

${ }^{5}$ Pittsburgh, PA; ${ }^{6}$ Durham, NC; ${ }^{7}$ Cambridge, MA

BACKGROUND: Meningococcal vaccines against serogroups A, C, W-135 and Y are recommended for routine use in adolescents in the United States (US), some Canadian provinces, and high-risk persons in other age groups in both countries.

METHODS: A randomized phase 3 trial at 67 clinical centers in the US and Canada assessed the immunogenicity and tolerability of MenACWY-CRM versus MCV4 in 2-to-10-year-olds. Eligible 2- to 5 -year-olds received (1:2:2): 2 doses of MenACWY-CRM or 1 dose of MenACWY-CRM or MCV4; 6-10 year olds received (1:1) 1 dose of MenACWY-CRM197 or MCV4. The primary immunogenicity assessment was seroresponse among the two age cohorts 28 days following a single dose of either vaccine. Noninferiority and superiority criteria were predefined. Solicited injection-site and systemic reactions were evaluated for 7 days postvaccination.

RESULTS: 2907 children participated. The percentage of MenACWYCRM recipients with seroresponse was statistically noninferior versus that in the MCV4 group for serogroups C, W, and Y in each age cohort, but all serogroups in the full population. The percentage of children with seroresponse to serogroup A was $77 \%$ and $83 \%$ for MenACWYCRM and MCV4, respectively, in older children and $72 \%$ and $77 \%$ in the younger cohort. The seroresponse rate was statistically superior for MenACWY-CRM versus MCV4 for serogroups W and Y in 6- to 10 - year-olds. Safety parameters were overall similar across age cohorts and vaccine groups.

CONCLUSIONS: MenACWY-CRM197 and MCV4 were immunogenic and well tolerated in this study population.

\section{P009 \\ A COMPARISON OF THE WORLD HEALTH ORGANIZATION'S QUALITY ASSURANCE CRITERIA FOR ACUTE FLACCID PARALYSIS SURVEILLANCE IN CANADA TO OTHER COUNTRIES}

M Helferty, M Mitschke

Ottawa, ON

BACKGROUND: Elimination of indigenous wild poliovirus transmission was certified in the Americas in 1994. Currently, only four countries globally continue to have endemic transmission and consequently, the risk of importation remains. For this reason, Canada and other countries, continue to conduct active surveillance of acute flaccid paralysis (AFP) to monitor for potential imported cases of paralytic poliomyelitis virus.

PURPOSE: To compare Canada's non-polio AFP and adequate stool specimen collection rate based on the World Health Organization's
(WHO) AFP quality assurance criteria, to those of other countries from 2000 to 2009.

METHODS: Through the Canadian Paediatric Surveillance Program, active surveillance of AFP is conducted and forwarded to the Public Health Agency of Canada. A comparison of WHO's AFP quality assurance criteria was conducted using other countries that have the most similarities to Canadian AFP surveillance, including case ascertainment and case definitions.

RESULTS: In 2009, Canada met WHO's non-polio AFP target rate of greater than $1 / 100,000$ cases in persons less than 15 years of age for the first time since 2000. Compared to Australia (range of 0.7/100,000 to $1.5 / 100,000$ ) and Greece (range 0.7/100,000 to 1.3/100,000), Canada's rate is similar. Endemic countries that have more robust surveillance systems such as India (range $6.4 / 100,000$ to $11.1 / 100,000$ ) and Afghanistan (range 1.1/100,000 to $8.6 / 100,000$ ) have significantly increased rates. An adequate stool collection rate of $80 \%$ within 14 days of symptom onset has not been achieved in Canada or Australia since 2000. Greece has met WHO's target once. India has consistently met this target and Afghanistan has continued to meet it since 2002.

CONCLUSION: Endemic countries that have more vigorous surveillance have continually experienced a higher incidence of AFP as compared to non-endemic countries. Continuous active surveillance, including more systematic stool collection methods in conjunction with high immunization coverage rates is essential to maintain Canada's poliovirus-free status.

\section{P010}

\section{EPIDEMIOLOGY OF INVASIVE PNEUMOCOCCAL DISEASE (IPD) IN NORTHERN CANADA, 1999 TO 2009}

\section{$M$ Helferty \\ Ottawa, ON}

BACKGROUND: The International Circumpolar Surveillance (ICS) initiative is a population-based invasive bacterial disease surveillance network of circumpolar countries. Within Canada, five regions and a network of laboratories participate in this initiative. IPD has been under surveillance since 1999 .

PURPOSE: To describe the epidemiology of IPD in Northern Canada from 1999 to 2009.

METHODS: Laboratory-confirmed cases are reported to the Public Health Agency of Canada (PHAC) by communicable disease officers in the respective jurisdictions. A completed standardized case report form and laboratory data are forwarded for analysis.

RESULTS: A total of 394 laboratory-confirmed cases, including 329 hospitalizations and 23 deaths of IPD were reported between 1999 and 2009. Annual population-based incidence rates fluctuated from a low of $15.8 / 100,000$ in 2005 to a high of $35.1 / 100,000$ in 2001 . The majority of IPD cases were in persons of aboriginal origin.

From 1999 to 2008, the highest incidence rates have consistently been among 0 to 4 year olds (range: 24.0 to $127.9 / 100,000$ persons) followed by 65 years and older (range: 29.3 to $87.8 / 100,000$ persons); however, in 2009, this trend reversed. The incident rate in cases 0 to 4 years of age was $85.7 / 100,000$ in 2008 compared to $77.9 / 100,000$ in 2009 . The incident rate in cases greater than 65 years in 2008 was $85.7 / 100,000$ compared to $93.6 / 100,000$ in 2009.

Serotypes covered within PCV-7 have decreased since vaccine implementation and account for less than $28 \%$ of infections. Serotypes covered in PCV-13 have been reported more frequently in all years (range: $19 \%$ in 2009 to $86 \%$ in 2001) except in 2008; serotypes in the PPV23 were reported most (42\%). In 2009, $52 \%$ of infections were caused by serotypes not covered in any vaccine.

CONCLUSION: The epidemiology of IPD has changed over this surveillance period. The most pronounced changes are in age group and serotype distribution. 


\section{P011}

\section{EPIDEMIOLOGY OF HAEMOPHILUS INFLUENZAE (HI) IN NORTHERN CANADA, 2000 TO 2009}

\section{Helferty}

\section{Ottawa, ON}

BACKGROUND: The International Circumpolar Surveillance (ICS) initiative is a population-based invasive bacterial disease surveillance network of circumpolar countries. Within Canada, five regions and a network of laboratories participate in the ICS initiative. Hi has been under surveillance since 2000.

PURPOSE: To describe the epidemiology of Haemophilus influenza in Northern Canada from 2000 to 2009.

METHODS: Cases that meet the national case definition are reported to respective jurisdictions where a communicable disease officer completes a standardized case report form and forwards the isolate to a reference laboratory. The completed case report form and laboratory results are then forwarded to the Public Health Agency of Canada (PHAC).

RESULTS: A total of 106 laboratory-confirmed cases, including 99 hospitalizations and 5 deaths, of $\mathrm{Hi}$ were reported to PHAC between 2000 and 2009. The majority of cases were in persons of aboriginal origin.

Annual population-based incidence rates ranged from a high of $12.5 / 100,000$ in 2001 to a low of 4.2/100,000 in 2003. The greatest burden of disease was consistently highest among 0 to 4 year olds (range $24.0 / 100,000$ to $111.9 / 100,000)$.

Overall, Hib immunization coverage rates varied yearly with the highest uptake rate (92\%) occurring in 2007 and the lowest (44\%) in 2001.

Serotype 'a' was most prevalent throughout the surveillance period (range: $29 \%$ in 2008 to $59 \%$ in 2001), however; in 2008, type 'b' resulted in more cases (43\%). Infections caused by other serotypes varied. Type 'b' ranged from $0 \%$ in 2000 to $43 \%$ in 2008 while infections due to 'non-typeable' ranged from $8 \%$ in 2007 to $38 \%$ in 2000.

CONCLUSION: Throughout the surveillance period, the epidemiology of Hi has not seen a marked change in Northern Canada except in 2008 with respect to serotype distribution. Active surveillance remains important for early detection of changing disease patterns and for monitoring immunization programs.

\section{P012}

\section{A SURVEY ON SEROGROUP B ISOLATES FROM INVASIVE MENINGOCOCCAL DISEASE (IMD) CASES IN THE} CANADIAN PROVINCE OF ONTARIO FROM 2001 TO 2008 D Law $^{1}$, F Jamieson ${ }^{2}$, P Rawte ${ }^{2}$, S Brown ${ }^{2}$, S Deeks ${ }^{2}$, J Zhou ${ }^{1}$, S Deng1, R Tsang ${ }^{1}$

\section{${ }^{1}$ Winnipeg, MB; ${ }^{2}$ Toronto, ON}

BACKGROUND: Invasive meningococcal disease (IMD) due to serogroup B isolates remains a significant threat to the health of Ontarians, in particular since the introduction of conjugate vaccines against other IMD serogroups (A, C, Y, W135).

OBJECTIVE: To analyse the genetic subdivisions of all the serogroup B isolates in Ontario from 2001 to 2008, and review the identified clonal complexes and sequence-types and demographic associations (age).

METHODS: Serogroup B IMD isolates from 2001-2008 were analysed by age and multi-locus sequence typing (MLST) to assign a genetic subgroup, according to their sequence types (STs).

RESULTS: 145 serogroup B IMD case isolates in Ontario from 2001 to 2008 were included. They were classified into 69 different STs; 57 STs can be grouped into 14 different clonal complexes, while 12 STs could not be assigned to any known clonal complex. Among the 14 clonal complexes, four dominant clones accounted for about $69 \%$ of all serogroup B cases. The most common clone was the ST-41/44 clonal complex or lineage 3 ( 54 cases or $37.2 \%$ ), followed by 2 related STs (ST-5571 and ST-336) which could not be assigned to any known clonal complex (20 cases or $13.8 \%$ ), ST-269 clonal complex (16 cases or $11 \%$ ) and ST-32/ET15 clonal complex (10 cases or $6.9 \%$ ). The ST-41/44 clonal complex was observed in mostly younger children while three other predominant clonal complexes, ST-5571 and ST336, ST-269 and ST32/ET-5 seemed to be mainly affecting adolescent and adults.

CONCLUSIONS: In contrast to the conventional view that serogroup B IMD mainly affects infants and the elderly, certain clones have been identified that appear to cause invasive disease primarily in adolescents and young to middle age adults. It is only through development, licensing and implementation of vaccines against serogroup B disease that IMD will be controlled in Canada.

\section{P013}

THE EPIDEMIOLOGY OF MEASLES IN CANADA, 2002-2009

\section{Y Li, K Grimsrud, K Mansfield}

Ottawa, ON

BACKGROUND: Canada's goal of measles elimination was endorsed by the Conference of Deputy Ministers of Health in 1995. Since the introduction of routine two-dose immunization and catch-up campaigns in 1996/97, the average annual incidence of measles has declined by 96\%.

PURPOSE: This study presents the overall epidemiology and elimination status of measles in Canada from 2002 to 2009.

METHODS: The measles data from 2002 to 2009 was collected through the Canadian Measles/Rubella Surveillance System (CMRSS) by weekly reporting from all provinces and territories to the Public Health Agency of Canada. A confirmed case was defined according to the Case Definitions for Communicable Diseases under National Surveillance - 2000. The population data were extracted from the Statistics Canada CANSIM database. A descriptive analysis was conducted.

RESULTS: Between 2002 and 2009, a total of 227 confirmed cases of measles were reported in Canada, with an average of 11 cases annually except in 2007 and 2008. Outbreaks in 2007 in Quebec and 2008 in Ontario resulted in 96 and 53 cases, respectively. The average annual incidence rate between 2002 to 2009 was 0.9 per million population, compared to 1.9 per million population between 1998 to 2001. All cases were thought to be imported or importation related. $84 \%$ of the confirmed cases with an immunization history (145 out of 172 cases) had never been vaccinated and only $3 \%$ had received two-dose vaccination.

CONCLUSION: In Canada, indigenous transmission of measles has been eliminated by our current two dose childhood immunization schedule and high coverage rates (approximately 94\%). However, as expected, imported cases continue to occur and are sporadic. Secondary spread from these imported cases is self-limited and involves the few Canadians who are still vulnerable, especially children who have never been vaccinated or who have received less than two doses of vaccine.

\section{P014}

CHANGING LOW ADULT IMMUNIZATION RATES IN CANADA: A PUBLIC SURVEY REGARDING FACILITATORS AND BARRIERS TO RECEIPT OF RECOMMENDED VACCINES AMONG CANADIAN ADULTS. A STUDY FROM CAN-AVERT (THE CANADIAN ADULT VACCINATION EVALUATION \& RESEARCH TEAM)

M Kiberd ${ }^{1}$, J Slaunwhite ${ }^{1}$, K Slayter ${ }^{1}$, B Halperin ${ }^{1}$, S Bowles ${ }^{1}$, S Halperin ${ }^{1}$, V Gilca ${ }^{2}$, E Dube ${ }^{2}$, C Sauvageau ${ }^{2}$, N Boulianne ${ }^{2}$, M Ouakki $^{3}$, F Lavoie $^{2}$, F Boucher ${ }^{2}$, I Gemmill ${ }^{3}$, SA McNeil ${ }^{1}$ ${ }^{1}$ Halifax, NS; ${ }^{2}$ Quebec, QC; ${ }^{3}$ Kingston, ON

BACKGROUND: Despite tremendous potential for disease prevention and reduction in healthcare costs, uptake for recommended adult vaccines remains low. The future holds great promise for more and better vaccines for adults. To ensure optimal protection through 
immunization, public health messaging must be responsive to information needs of the public and immunization programs must capitalize on factors that the public consider to be facilitators enabling easy vaccination and minimize barriers which adversely impact uptake.

METHODS: We conducted a nationwide web-based survey exploring attitudes \& behaviours of Canadian adults regarding recommended vaccines. Differences between provinces and by age were explored. Results are weighted by gender, age, region, language, and education to achieve representative sample.

RESULTS: 4,067 adults responded; $47.5 \%$ were $18-44$ y, 35\% 45-64y $\& 17.5 \% 65+y$. Influenza and hepatitis were most highly ranked by respondents as diseases with important health impacts (62\% and 20\%); less than $2 \%$ considered all other current immunization targets to be important. Less than $2 \%$ had ever been offered a vaccine or even knew that pertussis, HPV, pneumonia, and shingles could be prevented by immunization. Only $4.5 \%$ and $2.6 \%$ recall having ever been offered a tetanus or pertussis vaccine, respectively (versus $61 \%$ and $7.4 \%$ for influenza and hepatitis). $52 \%$ of respondents receive vaccines from their family doctor. $87 \%$ report that they visit a pharmacy $>$ once per year compared to only $59 \%$ who report regular visits to a physician.

DISCUSSION: Most adults are not aware of the disease impact of vaccine preventable infections, are not aware of available vaccines, and have never been offered most recommended adult vaccines. Delivery of vaccines by family physicians may limit accessibility, particularly among young adults who are less likely to have regular contact with a physician. Alternate delivery models, such as delivery by pharmacists may improve accessibility as most adults visit a pharmacy at least a few times per year. Strategies to optimize vaccine delivery need to be tailored differently for young healthy adults than for older adults or adults with comorbidities.

\section{P015}

\section{PUBLIC HEALTH RESPONSE TO DETECTION OF POLIOVIRUS IN TORONTO}

M Bartlett ${ }^{1}$, M Murti ${ }^{1}$, J Gubbay ${ }^{1}$, A Khan ${ }^{1}$, E Bontavics ${ }^{1}$, M Simpson ${ }^{1}$, T Booth ${ }^{2}$, S Dolman ${ }^{1}$, B Yaffe ${ }^{1}$, E Chong-King ${ }^{1}$, $S$ Desai $^{3}, M$ Finkelstein ${ }^{1}$

${ }^{1}$ Toronto, ON; ${ }^{2}$ Winnipeg, MB; ${ }^{3} \mathrm{Ottawa}, \mathrm{ON}$

BACKGROUND: Canada has been certified polio-free since 1995 but importation of wild poliovirus, oral polio vaccine virus (OPVV) and vaccine-derived polioviruses (VDPVs) presents an ongoing risk. In 2009, a 10-week old infant arrived in Toronto, Ontario from India via London with symptoms of fever and cough and was found to have OPVV type III on nasopharyngeal culture. The infant had received a second dose of oral polio vaccine (OPV - Sabin type III) in India eight days prior to departure.

PURPOSE: To review the detection of a child with OPVV and the public health response to this detection of poliovirus in Ontario.

METHODS: A retrospective chart review of the methods used by Toronto Public Health (TPH), the Ministry of Health and LongTerm Care (MOHLTC), the Ontario Agency for Health Protection and Promotion (OAHPP), the Public Health Agency of Canada (PHAC) and the National Microbiology Laboratory (NML) to investigate a case of OPVV detected in Ontario and the public health management of the case and contacts.

RESULTS: Strain analysis confirmed the OPVV with $<1 \%$ molecular sequence variation to the Sabin type III OPV strain. There was no evidence of secondary transmission to household contacts. Only one of nine household contacts and two of six non-household close contacts were found to be appropriately immunized against poliovirus. Information on vaccination was provided to all close contacts. TPH staff offered to immunize any close contact who requested vaccination. Four of nine household contacts were vaccinated by TPH staff and another three contacts received vaccine through their family doctor.

CONCLUSIONS: Given the risk of imported polioviruses (wild-type, OPVV and VDPVs) public health agencies at all levels must maintain capacity for detecting polioviruses and be prepared to cooperate closely in the investigation and management of these uncommon events.

\section{P016}

ADVERSE EVENTS FOLLOWING IMMUNIZATION (AEFI) WITH HUMAN PAPILLOMAVIRUS VACCINE AND HEPATITIS B VACCINES IN FEMALES AGED 9 TO 26 YEARS IN CANADA, 2006-2010

\section{J Nkanza, J Lafleche, H Anyoti, N Ahmadipour, R Pless, B Law Ottawa, ON}

BACKGROUND: Human Papillomavirus (HPV) and Hepatitis B virus (HBV) are two sexually transmitted infections for which vaccines are available, one recent (HPV-GardasilTM) and one with a long history (HBV-several). Both are often administered through school-based programs. This has led to controversy, thus continued safety monitoring is an important activity.

OBJECTIVE: To outline the safety monitoring of Gardasil ${ }^{\mathrm{TM}}$ postlicensure, present its AEFI profile, and compare it to HBV vaccines.

METHODS: AEFI reports for Gardasil ${ }^{\mathrm{TM}}$ and $\mathrm{HBV}$ vaccines (HB) were extracted from the Canadian Adverse Event Following Immunization Surveillance System. AEFI terms were listed and grouped using MedDRA (Medical Dictionary for Regulatory Activities) terminology. A serious adverse event is one that resulted in a hospitalization, prolongation of hospitalization, death, permanent disability, and/ or was life threatening.

RESULTS: Between July, 2006-May, 2010, 634 AEFI reports were received for Gardasil ${ }^{\mathrm{TM}}$ and 263 for HB. For another 72 , the vaccines were co-administered. HBV vaccine recipients were younger than those receiving Gardasil ${ }^{\mathrm{TM}}$ (12.2 vs 14.9 years). The proportion of serious events were similar between the two $\left(5.0 \%\right.$ of reports following Gardasil ${ }^{\mathrm{TM}}$ and $3.0 \%$ following $\mathrm{HB}$ ). Injection site reactions, fever, rash, and allergictype reactions were the most commonly reported terms for both vaccines $(10-13 \%)$. The most common major grouping terms were: General disorders and administration site conditions, Skin and subcutaneous tissue disorders, Gastrointestinal disorders and Nervous system disorders. For serious reports, the profiles were also similar.

CONCLUSION: The AEFI profile of Gardasil ${ }^{\mathrm{TM}}$ is very similar to that of HBV vaccines, which have been used in Canada for over a decade. From passive surveillance in Canada, and despite the intense publicity surrounding the HPV vaccine, no safety concerns have emerged.

\section{P017}

IMMUNOLOGIC EFFICACY OF PNEUMOCOCCAL VACCINE IN HIV PATIENTS: DELAYED VERSUS IMMEDIATE IMMUNIZATION

K Slayter $^{1}$, J Singer ${ }^{2}$, T Lee ${ }^{2}$, H Kayhty ${ }^{3}$, D Haase ${ }^{1}$, W Schlech ${ }^{1}$ ${ }^{1}$ Halifax, NS; ${ }^{2}$ Vancouver, BC; ${ }^{3}$ Helsinki, Finland

BACKGROUND: Invasive disease with Streptococcus pneumoniae is higher in adults infected with HIV. Little data are available to support recommendations for vaccine prevention of disease in this patient population. It is unclear whether immunization with a conjugate or a polysaccharide vaccine results in a better immunologic response and whether patients should be immunized immediately or delay vaccination until after reconstitution of the immune system.

METHODS: We conducted a multicentre controlled trial in which HIV positive patients were randomized to one of four arms: polysaccharide/immediate $(n=19)$, polysaccharide/delayed $(n=21)$, conjugate/ immediate $(n=23)$, and conjugate/delayed $(n=16)$. Proportional odds model was used to compare the groups (vaccine type and vaccine timing) on the number of serotypes showing response (2-fold rise in antibody level from pre-vaccination level) at 1, 6 and 12 months post-vaccination between the groups. Reactions to vaccination were compared between the two vaccines. 
RESULTS: 79 HIV patients were included in the trial. Baseline characteristics were similar for the four arms: $78 \%$ male, mean age 41 years, mean time since HIV diagnosis 2.26 year, mean CD4 count 79 cells $/ \mathrm{mm}^{3}$, and mean HIV viral load 107,152 copies $/ \mathrm{mL}$. For the delayed group, mean CD4 counts at vaccination was 180 cells $/ \mathrm{mm}^{3}$. Results in favor for delayed immunization were observed with respect to the number of serotypes showing response. The proportional odds ratios for delayed vs. immediate are $0.54(p=0.21), 0.341(p=0.04)$ and $0.204(\mathrm{p}=0.004)$ at months 1,6 and 12 , respectively. No differences were observed between the two individual vaccines. The difference between delayed and immediate was consistent across individual serotypes. Reactions to vaccination (pain, redness and swelling) were found to be more severe in the immediate group when compared to the delayed group.

CONCLUSIONS: These data provides evidence to support delaying immunization until after reconstitution of the immune system with pneumococcal vaccine. There was no increased immunological response when utilizing a conjugate based vaccine versus that of a polysaccharide vaccine.

\section{P018}

\section{EPIDEMIOLOGY OF NOSOCOMIAL ROTAVIRUS} INFECTIONS IN A CANADIAN PEDIATRIC HOSPITAL

\section{P Verhagen, D Moore, A Manges, L St-Martin, C Quach \\ Montreal, QC}

BACKGROUND: The prominent role of rotavirus (RV) as a pathogen in nosocomial gastrointestinal infections in pediatric settings has been recognized for several years.

PURPOSE: To determine incidence of nosocomial RV gastroenteritis (nRVGE), disease burden and characteristics of patients to support decision-making on potential vaccination strategies.

METHODS: We performed a retrospective cohort study of all nRVGE cases over a 10 -year period in a Canadian tertiary-care pediatric hospital. Cases were identified through the hospital's active prospective surveillance program for nosocomial infections. Patient characteristics and medical history, nRVGE symptoms and therapy were recorded.

RESULTS: A total of 214 cases were detected with an incidence rate of $0.5 / 1000$ patient-days (95\%CI: $0.43,0.57$ ) and $0.3 / 100$ admissions (95\%CI: $0.26,0.35)$ with no significant decline in rates over time. Infection rate was highest among patients with a hospital-stay of $>5$ days. nRVGE occurred in all age groups, but mostly in young infants (median: 8 months). A chronic underlying medical condition was present in 59\%, 3/4 had a history of previous hospitalization(s). In $44 \%$ chronic medical conditions were identified during the perinatal period. Median length of stay in hospital was 23 days (range: 4-2251). Median duration of diarrhea was 4 days (range 1-21). Rehydration was required for $132(62 \%)$ patients and was intravenous in 98 (46\%). The mean duration of IV catheterization was 3.3 days. Readmission because of nRVGE occurred in 26 patients $(12 \%)$ for a median duration of 4 days.

CONCLUSIONS: nRVGE continues to be an important problem in the Canadian pediatric hospital setting. It is predominantly a disease of children with chronic medical conditions who require recurrent and prolonged hospitalizations. Disease burden is significant with nearly half requiring IV hydration. Vaccination, either through a universal immunization program or targeted immunization for high-risk patients, could have significant impact on nRV disease burden.
P019

NOSOCOMIAL ROTAVIRUS GASTROENTERITIS: POSSIBLE IMPACT OF A TARGETED IMMUNIZATION STRATEGY

\author{
P Verhagen, A Manges, C Quach \\ Montreal, QC
}

BACKGROUND: Rotavirus (RV) is the leading cause of severe gastroenteritis $(\mathrm{GE})$ in young children worldwide. RV is a recognized nosocomial pathogen on pediatric wards. In the Canadian hospital setting, nosocomial RVGE (nRVGE) affects mainly patients with underlying chronic medical conditions requiring frequent and prolonged hospitalizations. The impact of nRVGE on these patients can be substantial. Because of concerns about cost-effectiveness, Canada does not currently have a universal RV immunization program.

PURPOSE: As an alternative, we assessed the possible impact of a targeted immunization strategy on reducing nRVGE in specific highrisk patients.

METHODS: Data were derived from a recent cohort study on incidence and patient characteristics of nRVGE in a Canadian Pediatric Hospital. "High-risk" patients were defined as those suffering from medical conditions identified early in life and often associated with recurrent hospitalizations. RV vaccination at 2, 4 (and 6) months was modeled for high-risk patients. The transition probabilities were based on age distribution of nRVGE cases and published vaccine effectiveness data.

RESULTS: Over a 10-year period of observation, 95 cases of nRVGE (44\%) occurred in high-risk patients eligible for vaccination. A targeted immunization program could prevent approximately half of nRVGE (49\%) in the high-risk group and about a quarter of all cases of nRVGE (22\%). Almost $60 \%$ of severe cases of nRVGE could be prevented in the high-risk group and $25 \%$ in the entire cohort. Based on the incidence estimate for nRVGE of $0.5 / 1000$ patient-days, 566 to 750 cases occur each year across Canada. The number prevented by targeted immunization would be 100 to 210 per year.

CONCLUSION: Targeted immunization could generate an important reduction in nRVGE among "high-risk" patients. While limiting costs compared to universal vaccination, a targeted vaccination strategy could prevent additional morbidity due to nRVGE in vulnerable patients groups and reduce nosocomial infection rates.

\section{P020}

DESCRIPTIVE EPIDEMIOLOGY OF INVASIVE PNEUMOCOCCAL DISEASE IN ONTARIO CHILDREN (2008-2009): A PUBLIC HEALTH DATABASE APPROACH

\section{A Wormsbecker, A McGeer, R Olsha, J Gubbay}

Toronto, ON

BACKGROUND: Heptavalent pneumococcal conjugate vaccine (PCV7) reduced rates of pediatric invasive pneumococcal disease (IPD) but non-PCV7 serotype (ST) IPD is a concern.

PURPOSE: Goals were to use the Ontario Ministry of Health and Long-Term Care's (MOHLTC) integrated Public Health Information System (iPHIS) to, in the context of a universal PCV7 vaccination program, determine the present incidence of pediatric IPD in Ontario and to describe cases by clinical and demographic characteristics, including Public Health Unit (PHU) Region, ST, and vaccine status.

METHODS: IPD cases from January 2008 to June 2009 (age $<15$ years) were extracted from iPHIS. Missing ST and vaccine information were obtained from the Ontario Agency for Health Protection and Promotion (OAHPP) Public Health Lab (PHL) and the Toronto Invasive Bacterial Diseases Network (TIBDN) when possible.

RESULTS: The incidence of pediatric IPD was 7.34/100 000 person years $(243$ cases) and there were no differences among PHU regions $(p>0.05)$ or between sexes $(p>0.05)$. Twenty-five cases $(10.0 \%)$ had record of attending daycare and 42 cases $(17.3 \%)$ had chronic medical conditions. Vaccine information was available for 127 cases $(52.3 \%)$ 
and only 2 of 19 cases of PCV7 ST IPD had record of complete PCV7 vaccination. ST information was available for 187 cases (77.0\%). NonPCV7 STs, primarily additional thirteen-valent pneumococcal vaccine (PCV13) STs and non-conjugate vaccine STs, were more common than PCV7 STs. The highest rate by age and serotype was PCV13 ST IPD in 12-23 month olds (14.3/100 000 person years).

CONCLUSION: Present pediatric IPD rates are low and the STs isolated suggest replacement by non-PC7 STs. However, the quality of these data is poor regarding clinical and demographic characteristics. For example, Toronto Public Health estimates that $60 \%$ of Toronto children attend daycare while only $10 \%$ of this province-wide group did, suggesting under-recording of risk factors. Vaccine information is also limited. Improving data quality, especially that of vaccination status, in this public health surveillance system would assist in the evaluation of vaccination programs.

\section{IMMUNIZATION PRACTICE AND INNOVATION} OPPORTUNITY

\section{P021}

\section{ASSESSMENT OF IMMUNIZATION SERVICES DELIVERY AT PRIVATE SECTORS IN DUBAI}

AH Al Marzooqi, HA Al Khateeb, Al El Gak

Dubai, United Arab Emirates

BACKGROUND: The role of private-sectors in the provision of health care services is increasing in Dubai. About $70 \%$ of health care services provided by private sectors.

PURPOSE: This current study aims to assess scope of practice and quality of immunization services provided at private health care facilities, and to identify opportunities for the development of policy and guidelines as part of health care quality assurance within Dubai Health authority.

METHODS: The three main sources of information included a survey questionnaire accompanied by direct observation of clinical practices and review of national and international literature. A questionnaire survey was conducted at 50 private facilities selected randomly from different areas of Dubai.

RESULTS: All studied private health care facilities provided immunization services. $60 \%$ followed national immunization guidelines while $24 \%$ followed other guidelines. Although the private health care facilities demonstrated high level immunization records, the reminder and follow up system was not adequate. $76 \%$ of the private clinics have no clear methods of communication with their clients. With regard to adverse event reporting, the study revealed only $56 \%$ of health care facilities has included adverse events in their reporting system. However, in $96 \%$ of vaccination clinics the parents were informed. $77 \%$ of private clinics assigned special staff for immunization. $90 \%$ of the private clinics followed specific policy to maintain cold chain. There was no standard fridge for vaccine storage in almost $40 \%$ of the studied clinics. In other $60 \%$ with standard fridge, there was high compliance with the regular monitoring of vaccine-storage temperature.

CONCLUSION: The quality of immunization services provision was not adequate in terms of policy and national guidelines to be followed, recording and reporting of vaccination practices. Policy and operational guidelines are required for private sector immunization practices that address critical subject areas.
P022

IMPLEMENTATION OF REQUIRED CONTINUING COMPETENCY PROGRAM FOR REGISTERED NURSES PROVIDE A UNIQUE OPPORTUNITY TO RECRUIT COMMUNITY HEALTH NURSES FOR EDUCATION SESSIONS REGARDING THE IMMUNIZATION COMPETENCIES FOR HEALTH PROFESSIONAL DOCUMENT, 2008

\section{PA Allan}

Corner Brook, NL

BACKGROUND: In 2009 the Association of Registered Nurses of Newfoundland and Labrador (ARNNL) announced that as of April 1, 2010, registered nurses in the province of NL would be required to commence documentation of their continuing education to maintain their competency. Nurses need to participate in 14 hours of continuing education to maintain competency and their licence to practice in the province. This new process has heightened awareness and interest among community health nurses in the western region. They are eager to ensure they obtain and document the required number of hours outlined by the provincial nursing body, the ARNNL. The recent release of the Immunization Competencies for Health Professional Document, 2008, has provided and a unique educational opportunity.

PURPOSE: The objective is to provide leaning opportunities for Community Heath Nurses about immunization to enhance their knowledge and skill while meeting their need for continuing competency. METHODS: To recruit Community Health Nurses for educational sessions to enhance knowledge and skills related to immunization competencies. Each nurse was provided with their own copy of the Immunization Competencies for Health Professional, 2008. Information on both the ARNNL continuing competency program as well as the teleconference sessions was provided to the nurses to gain their interest. The sessions commenced in May when Community Nurses had identified their learning needs through the ARNNL competency program. A pre and post questionnaire was distributed to staff to help determine if they increased their knowledge level during the in-service.

RESULTS: Staff attendance at the conference calls is high and is being documented and staff will receive a certificate upon completion to confirm their continued competency hours. A pre and post questionnaire was distributed to staff to determine if they increased their knowledge level.

CONCLUSION: The Immunization Competencies Document for Health Professionals, 2008, provides clear detailed specific information about the competencies needed to provide immunizations. Utilizing the need identified by the ARNNL nurses governing body had created an opportunity to increase immunization competencies.

\section{P023}

\section{VACCINE DELIVERY PROJECT USING TEMPERATURE CONTROLLED ENVIRONMENT VEHICLES}

E Stillo, S Kucinska De Ocampo, E Teoh, L Esguerra, R Mowat, M Gillespie, M Varia, A Ashton

Brampton, ON

BACKGROUND: The Region of Peel is the second largest municipality in Ontario with a population of over 1.2 million residents in the cities of Mississauga, Brampton, and the town of Caledon. Peel Public Health services an estimated 1,200 physicians and distributes over 1.1 million doses of vaccine on an annual basis. Vaccine pick up and transport is the responsibility of each physician and is conducted using an approved hard sided cooler and associated cold chain protocols. Due to the geographic spread of the Region, some physicians travel up to 30 kilometers to pick up vaccine from the nearest health unit office. These practices have expressed concerns related to the distance and time investment required to obtain vaccine for the immunization 
of their patients. Other physicians find the cold chain protocols for vaccine transport challenging.

PURPOSE: The purpose of this project is to evaluate the use of temperature controlled vehicles for vaccine delivery in increasing accessibility to publicly funded vaccines within Region of Peel while maintaining vaccine cold chain.

METHODS: Vaccine is transported to physician practices using temperature controlled vehicles that maintain and monitor cold chain temperatures.

RESULTS: During the 2009/2010 influenza season, approximately 300,000 doses of $\mathrm{pH} 1 \mathrm{~N} 1$ vaccine were delivered (at no cost to the client) over an 8 week period using temperature controlled vehicles. Of 891 delivered packages, 10 (1\%) packages were affected by a cold chain failure. Informal feedback confirmed physician satisfaction with the delivery service. Post H1N1, physicians are now offered the option to continue delivery on a fee-for-service basis.

CONCLUSION: Preliminary results from H1N1 confirmed demand and provided direction to further develop the vaccine delivery service. Data presented will address the feasibility and effectiveness of the vaccine delivery project and will provide insight into a strategy that may be transferable to other local health units.

\section{P024}

IS AN OUNCE OF PALIVIZUMAB WORTH A POUND OF CURE?

A Banerii ${ }^{1}$, M Young ${ }^{2}$, D Bisson ${ }^{3}$, L Giles ${ }^{4}, \mathrm{C}$ Huii $^{5}$, K Lanctot ${ }^{1}$, B Lee $^{6}$, J Mahony ${ }^{7}$, C Majaesic ${ }^{6}$, J Morel ${ }^{8}$, B Paes ${ }^{7}$, J Robinson 6

${ }^{1}$ Toronto, ON; ${ }^{2}$ Yellowknife, NT; ${ }^{3}$ Churchill, MB; ${ }^{4}$ Winnipeg, MB; ${ }^{5}$ Ottawa, ON; ${ }^{6}$ Edmonton, $\mathrm{AB} ;{ }^{7}$ Hamilton, $\mathrm{ON}$; ${ }^{8}$ Montreal, QC

BACKGROUND: Hospitalization rates for lower respiratory tract infections (LRTI) within Arctic communities are extremely high with rates of 300/1000 to 600/1000 infant-year with the majority due to respiratory syncytial virus (RSV). Due to the high rates of hospitalizations in the Arctic, where infants are typically evacuated by air, RSV has a disproportionate health and financial impact for the Inuit.

PURPOSE: To establish a baseline for rates of LRTI and RSV, to identify viruses associated with LRTI admissions in children less than one year of age who reside in the Arctic, and perform a budget impact analysis for enhanced palivizumab prophylaxis in remote communities of Inuit infants of all gestational ages who are less than 6 months at the start of RSV season.

METHODS: Active surveillance over an 18 month period of infants less than a year of age who reside in communities in Northwest Territories, Western Nunavut and Nunavik, with admissions for LRTI. Rapid RSV diagnostic tests were performed on NPA samples taken upon admission. Specimens were processed with a 19 panel multiplex PCR using Luminex ${ }^{\circledR} 100^{\mathrm{TM}}$. Demographics, costs data for inpatient hospitalization and palivizumab data were also collected.

RESULTS: There were 300 cases enrolled, where $14.6 \%$ qualified as palivizumab candidates. Viruses were identified in $95 \%$ of analyzed samples with one virus identified in $69.6 \%$ specimens, and $26.5 \%$ specimens with multiple infections. RSV was identified in $55 \%$ of the cases. The admissions were associated with high costs, prolonged hospitalizations and high rates of ICU admissions. Budget impact analysis demonstrated that the use of palivizumab would decrease the burden and costs associated with RSV admissions.

CONCLUSION: RSV was positively identified within a significant majority of NPA samples. The high rates of admission and the accompanying hospital costs support the new CPS guidelines where Inuit infants of all gestational ages from Arctic communities should be prophylaxed with palivizumab.

\section{P025}

EFFICACITÉ D'UNE RELANCE POSTALE EN VUE D'AMÉLIORER LA COUVERTURE VACCINALE À 24 MOIS N Boulianne, M Kiely, D Audet, C Couture, R Maranda-Aubut, G Deceuninck

Québec, QC

CONTEXTE : Au Québec, la couverture vaccinale à 24 mois n'atteint pas l'objectif de $95 \%$ fixé dans le Programme national de santé publique. Ce sont principalement les vaccins recommandés à l'âge de 18 mois qui font diminuer la couverture vaccinale. L'intervention de rappel est une stratégie reconnue efficace pour améliorer les couvertures vaccinales. L'efficacité et la faisabilité de cette stratégie n'ont pas été évaluées au Québec.

BUT : L'étude visait à évaluer l'efficacité d'une relance postale pour améliorer la couverture vaccinale à 24 mois et pour diminuer les retards vaccinaux.

MÉTHODES : Les enfants âgés entre 15 et 16 mois ont été identifiés à l'aide du fichier régional de vaccination de la région de Québec entre le 1er juin 2009 et le 5 avril 2010. 1715 enfants ont été randomisés en deux groupes égaux, un groupe d'intervention et un groupe contrôle. Les parents du groupe d'intervention ont reçu une lettre de relance personnalisée les invitant à prendre un rendez-vous pour les vaccins de 18 mois. Le statut vaccinal à 19 mois ainsi que l'âge à la vaccination ont été évalués dans un premier temps.

RÉSULTATS : Des résultats préliminaires étaient disponibles pour 1103 enfants âgés de 20 mois ou plus au 1er mai 2010. Les enfants dans le groupe de relance étaient plus nombreux à avoir reçu leur $4 \mathrm{e}$ dose de DCaT-P-Hib* et leur 2e dose de RRO** avant l'âge de 19 mois comparativement à ceux du groupe contrôle $(66 \%$ versus $57 \%$ p=0,003). Parmi ceux qui ont reçu les vaccins prévus à 18 mois, l'âge à la vaccination était inférieur dans le groupe d'intervention comparativement au groupe contrôle (18,8 mois versus 19 mois; $\mathrm{p}=0,01)$

CONCLUSIONS : Selon les analyses intérimaires de cette étude, l'intervention de relance réalisée a permis d'améliorer les couvertures vaccinales pour les vaccins prévus à 18 mois.

*Vaccin contre la dipthérie, la coqueluche, le tétanos, la poliomyélite et Haemophilus Influenzae de type b.

**Vaccin contre la rougeole, la rubéole et les oreillons.

\section{P026 \\ PUBLIC HEALTH AGENCY OF CANADA'S YELLOW FEVER VACCINATION PROGRAM}

\section{Bryson, C Sivaraj, J Geduld}

Ottawa, ON

BACKGROUND: The Public Health Agency of Canada's (PHAC) Yellow Fever Vaccination Program is responsible for designating Yellow Fever Vaccination Centres (YFVC) in Canada. YFVC are official locations where patients can receive the yellow fever vaccination. As a signatory of the International Health Regulations (IHR) (2005), Canada is required to designate specific YFVC to help prevent, protect and control the spread of yellow fever and to facilitate Canadian travellers crossing international borders. Yellow fever is currently the only disease under the IHR (2005) for which proof of vaccination may be required as a condition of entry into a country.

PURPOSE: To inform health care providers of the Yellow Fever Vaccination Program and the designation process of YFVC in Canada. To summarize the status of YFVC in Canada and highlight the relevance to Canadian travellers and health care providers.

METHODS: A descriptive analysis of data available from the Yellow Fever Vaccination Program and the World Health Organization was performed. Statistics Canada data were used to describe patterns of travellers from Canada to countries with yellow fever vaccination requirements.

RESULTS: As of May 1, 2010, there were 420 clinics designated as YFVC in Canada; 74 (17.6\%) were designated in the past year. 
The WHO publishes a list of locations that require proof of yellow fever vaccination for entry. Of the 193 countries and 32 territories listed, 19 (8.4\%) require proof of yellow fever vaccination for all travellers; 132 (58.7\%) require proof for travellers that have visited a country with risk of transmission.

Between 2000 and 2008, there was an 88.4\% increase in Canadian visits to countries with yellow fever entry requirements for which data were available.

CONCLUSION: Because more Canadians are travelling to countries with yellow fever vaccination entry requirements, it is important for health professionals to be aware of the process for administering this vaccine in Canada.

\section{P027}

REGIONAL AND PROVINCIAL SURVEILLANCE STRATEGIES TO ENSURE REAL TIME REPORTING OF ADVERSE EVENTS FOLLOWING IMMUNIZATION TO THE PUBLIC HEALTH AGENCY OF CANADA

PA Allan ${ }^{1}$, GA Butler ${ }^{2}$

${ }^{1}$ Corner Brook, NL; ${ }^{2}$ St. John's, NL

BACKGROUND: Monitoring of Adverse Events Following Immunization (AEFI) is essential to ensure that unusual events are recorded and reported allowing for prompt analysis and interventions. The introduction of a new vaccine, $\mathrm{pH} 1 \mathrm{~N} 1$, during a mass vaccination campaign presented an increased likelihood of adverse events.

PURPOSE: Prompt reporting of Adverse Events Following Immunization produces up-to-date data collection and surveillance. This supports the Vaccine Safety Section, Centre for Immunization and Respiratory Infectious Diseases (CIRID) in monitoring unusual events, enhancing reporting and analysis of vaccine safety data within Canada.

METHODS: Numerous strategies were implemented regionally and provincially during the H1N1 planning phase to ensure timely and documented reports of adverse events. In 2006 Newfoundland and Labrador (NL) participated in a pilot project to test a new AEFI report form. A training session was provided by CIRID at a provincial immunization conference in 2007. When the mass immunization clinics began in the fall of 2009, weekly conference calls were held to discuss AEFIs that occurred after the $\mathrm{pH} 1 \mathrm{~N} 1$ vaccination. All vaccine recipients were provided with contact numbers and urged to report unusual events associated with the vaccine. All subsequent reports were faxed to provincial office. The jurisdictional safety lead was notified by phone of all urgent events that required action, in turn the Chief of Vaccine Safety/designate was immediately notified.

RESULTS: The provincial mass immunization registry database allowed access to $\mathrm{pH} 1 \mathrm{~N} 1$ vaccine lot numbers and data relating to vaccine administration. The provincial HealthLine was used as a screening and referral triage. Accurate and timely recording of this information was essential for analysis to take place at all levels. Weekly jurisdictional aggregate reporting of $\mathrm{pH} 1 \mathrm{~N} 1$ vaccine AEFIs was done by the jurisdictional safety lead. All strategies implemented assisted the VVWG committee and CIRID in monitoring the safety of the vaccine.

CONCLUSION: There were 264 AEFI reports following the pH1N1 vaccine for the province. The process established in Newfoundland and Labrador assisted in the monitoring of the safety of this new product thus enhancing vaccine safety for the Canadian public.
P028

CREATING A COALITION OF SUPPORT: HUMAN PAPILLOMAVIRUS VACCINE PROMOTION EVENTS IN BRITISH COLUMBIA COMMUNITIES

\author{
B Deeter $^{1}$, S Gorringe ${ }^{1}$, K Pielak ${ }^{1}$, G Ogilvie ${ }^{1}$, P White ${ }^{1}$ \\ Vancouver, BC
}

BACKGROUND: Human Papillomavirus vaccine uptake rates in British Columbia (BC) were among the lowest in the country at $62 \%$ (grade 6) and 62.5\% (grade 9) for the 2008-2009 school year.

Research indicates that an important modifiable factor in parental decision making about HPV vaccine is the recommendation of a trusted health professional (Ogilvie et al., 2010). Understanding that concerns about efficacy, safety, and potential health impact of vaccination are primary factors driving health professionals' decision to recommend the vaccine (Khan, 2007), a plan was made to address these issues directly with immunization influencers.

PURPOSE: To investigate whether educational events with local experts could impact immunization provider confidence and intention to recommend the HPV vaccine to patients.

METHODS: Local cancer experts were targeted and trained to partner with public health in the provision of education events where they could publicly support the vaccine. The events featured additional experts on safety and efficacy (Medical Health Officers) and on HPV immunization communication (Public Health Nurses).

Events were held throughout $\mathrm{BC}$ with local physicians, nurses and pharmacists attending. Community physicians were offered continuing medical education credits for attending the session. Evaluation includes measurement of both the short and longer term impact of this intervention on provider confidence, ongoing intention to recommend, and actual recommendations made.

RESULTS: $98 \%$ of attendees ( $n=158 ; 43$-Physicians, 6-Nurse Practitioners, 18-Pharmacists, 2-midwives, 80 Nurses, 9 unknown) found the event "useful" or "very useful" and $88 \%$ said they feel more confident discussing vaccine with parents and girls. $87 \%$ committed to speaking with 5 people about the benefits of the HPV vaccine in the next month.

CONCLUSION: Local expert-led events can raise health care provider confidence and intention to engage with patients about HPV vaccine in the short term. Additional evaluation is ongoing to determine what the longer term impact of these events will be.

\section{P029}

IMMUNIZATION UPTAKE IN A HARD TO REACH HIGH RISK POPULATION - AN UNEXPECTED BENEFIT OF POINT OF CARE (POC) HIV TESTING

HJ Winnichuk ${ }^{1}, \underline{C}$ Demarinis $^{2}$

${ }^{1}$ New Westminster, BC; ${ }^{2}$ North Delta, BC

BACKGROUND: Homeless and street-entrenched individuals are some of the most vulnerable high-risk individuals who could benefit from immunization. However, they may also be a very difficult population to reach. In 2008, Fraser Health submitted a proposal to the BC Ministry of Healthy Living and Sport for a pilot project to increase testing for HIV among those who have limited access to primary care and those who engage in HIV risk activities. The funding we received enabled public health nurses working on the HIV team to offer outreach services including immunization to this population between January 2009 and January 2010.

PURPOSE: To describe the uptake of immunization among individuals who were approached for POC HIV testing.

METHODS: The public health HIV nurses in Fraser Health partnered with local non-profit agencies in a variety of settings (food banks, hot lunch programs, churches, mobile outreach van) to offer POC HIV testing and other outreach services including immunizations. 
RESULTS: 353 clients were seen by the POC Outreach nurses. 144 clients received at least one vaccine with a total of 341 immunizations given during the year.

CONCLUSION: Although funding was targeted for POC HIV testing, an unexpected benefit was the number of high risk individuals vaccinated who typically would not have accessed immunization services. When asked if they would have tested for HIV elsewhere, 90\% responded no. Contact with these clients allowed public health HIV nurses to assess and immunize these individuals with vaccines they were eligible for but had not previously received. Relationships were established between public health HIV nurses, local non-profit agencies and hard to reach clients that allowed for ongoing follow up and completion of vaccine series. As well, these relationships enabled public health to access this priority population promptly during the H1N1 pandemic to administer vaccine.

Due to the success of the pilot project, Fraser Health has continued to support outreach services to this population.

\section{P030 \\ HIGH RISK NEONATAL HEPATITIS B IMMUNIZATION AND FOLLOW-UP}

\section{$\underline{C}^{\text {Demarinis }}{ }^{1}, \underline{\mathrm{H} \text { Winnichuk }}{ }^{2}$}

${ }^{1}$ North Delta, BC; ${ }^{2}$ New Westminster, BC

BACKGROUND: Fraser Health is committed to reducing the risk of neonatal Hepatitis B transmission. In order to follow provincial recommendations regarding post exposure prophylaxis (PEP), the Fraser Health Communicable Disease Team developed a protocol to ensure follow up is completed on all high risk infants. This protocol allows for a consistent approach regardless of which health care discipline is providing the follow up (public health nurse, physician, midwife).

The Neonatal Hepatitis B Program incorporates intense follow up of high risk infants to ensure that they receive the appropriate prophylaxis and testing.

PURPOSE: To reduce the risk of neonatal Hepatitis B transmission by using a systematic approach with all identified high risk infants.

METHODS: Once high risk infants are identified, the established Fraser Health standard protocol is utilized to ensure a systematic approach to PEP and serological follow-up of high risk infants occurs. The protocol facilitates data collection and analysis of outcomes.

The presentation will outline the process and share supporting documents.

RESULTS: From 2008-2010, 313 infants were followed on the Neonatal Hepatitis B program. 30 infants were lost to Public Health follow up for various reasons (ie moved). Of the 313 infants in the program, 283 completed their Hepatitis B series. 5 of the 283 infants required a second series to obtain adequate protection. 28 of the infants who completed their Hepatitis B series did not have their serology done post vaccination; therefore, it is unknown if they developed adequate antibody levels.

CONCLUSIONS: Since implementation of the Fraser Health Neonatal Hepatitis B Program's standard protocol in 2007, there have been no cases of hepatitis $B$ infection in high risk infants who have completed follow up according to the protocol. $90 \%$ of these infants completed their Hepatitis B vaccine series.

\section{P031 \\ INCREASING PRESCHOOL IMMUNIZATION RATES IN LOW SOCIO-ECONOMIC NEIGHBOURHOODS, REGINA, SASKATCHEWAN}

M Granger, Z Abbas, D Churko, $\underline{\text { T Diener }}$

Regina, SK

INTRODUCTION: Immunization rates in the low socio-economic status (SES) neighbourhoods of the City of Regina remain lower than in the general population despite efforts to raise immunization levels in the city.
OBJECTIVE: To assess the effectiveness of providing targeted vaccination in day care centres in the low SES neighbourhoods and for clients attending the Regina Open Door Society (RODS), a centre for new immigrants and refugees, in raising immunization levels among children of low income families.

METHODS: Day care centres in the low-income areas of the City of Regina were selected. The percentage of children with up-to-date immunization was recorded at the time of enrolment in the study. The children were followed up for a period of six months. The percentage of children with up-to-date immunization was recorded at the end of the study. The main outcome measure was the percentage of children with up-to-date immunizations at the end of the study period.

RESULTS: A total of 227 children enrolled in four day care centres in the low SES neighbourhoods of the city and those attending RODS were enrolled in the study and were followed up for 6 months. Overall, the immunization coverage at the time of enrolment in the study was 65 percent. The immunization coverage at the end of the study period was 75 percent. The immunization completion rate was ten points higher at the end of the study period. The public health nurse made an average of two visits to each day care centre. The immunization level increased by 10 percent at a cost of $\$ 50$ per additional child immunized.

CONCLUSIONS: The intervention was effective at increasing immunization levels in low SES sub population who utilize day care centres. For targeted immunization programs and to raise immunization levels in low SES populations, it may be useful to employ methods that target a wide range of sub populations.

\section{P032}

\section{AUTOMATED IDENTIFICATION OF VACCINES PROJECTS} (AIVP): STANDARDS AND IMPLEMENTATION PROJECTS

\section{A Diniz, R Van Exan, L Belzak \\ Ottawa, ON}

BACKGROUND: In Canada, several million doses of vaccines are administered every year. Each time a dose of vaccine is administered, a health care provider manually records the details in a patient's health record. In 2001, audits in British Columbia and Manitoba showed that $15 \%$ of immunization records were incomplete, $24 \%$ contained errors and $5 \%$ were missing information.

Since 2002, the Public Health Agency of Canada (PHAC) and the Canadian Immunization Registry Network have been working with stakeholders to incorporate 2 -dimensional bar codes onto all vaccine products in Canada through the Automated Identification of Vaccines Project. The goal is to improve the efficiency and effectiveness of immunization delivery and the safe use of vaccines through the implementation of automated identification on vaccine products and to incorporate this technology into immunization registries.

PURPOSE: To promulgate the recently agreed upon standards for bar codes on vaccine products in Canada and the implementation projects for the next two years.

METHODS: Key stakeholders have been engaged in Automated Identification of Vaccines Projects since its inception 2002, and formally since 2007 through the AIVP Advisory Task Group. In February 2009, the bar code standards for vaccine products were agreed upon by stakeholders. Vaccine manufactures have agreed to implement these standards voluntarily over the next two years. Early adopter project plans are underway to assist in the implementation of these standards in both the public and private health care settings.

CONCLUSION: The Automated Identification of Vaccine Projects Advisory Task Group finalized and agreed upon the standards for bar codes on vaccine products in February 2009. The vaccine manufacturers have agreed to implement these standards on a voluntary basis over the next two years. The Advisory Task Group is working on a number of initiatives to encourage the use of this technology across Canada. 


\section{P033}

PROMOTION DE LA VACCINATION AU QUÉBEC : UN PLAN POUR L'ACTION

E Dubé $^{1}$, C Sauvageau ${ }^{1}, M$ Guay $^{2,3}$, N Boulianne ${ }^{1}$, G Petit $^{1,3}$

${ }^{1}$ Québec, QC; ${ }^{2}$ Longueuil, QC; ${ }^{3}$ Sherbrooke, QC

CONTEXTE : Le plan québécois pour la promotion de la vaccination a été publié en 2010. Neuf stratégies démontrées efficaces afin d'améliorer les couvertures vaccinales avaient été retenues; les lieux possibles d'interventions en découlant demeurent multiples.

OBJECTIFS : Identifier les interventions à implanter en priorité dans l'ensemble des régions du Québec.

MÉTHODES : Treize experts impliqués dans la promotion de la vaccination et provenant de différents paliers (provincial, régional, local) ont été consultés pour mettre les interventions en priorité à partir d'une grille de critères objectivables: ampleur du changement à apporter, acceptabilité par les vaccinateurs et la population, coûts humains et matériels.

RÉSULTATS : Au total, 12 interventions ont été retenues :

- Rappel de la vaccination aux parents de nouveau-nés lors du premier contact téléphonique en post-partum et prise du rendezvous pour la vaccination;

- Relance des enfants non vaccinés à 3 mois;

- Rappel et relance en $4 \mathrm{e}$ année du primaire;

- Ajout de plages horaires (incluant soir et fin de semaine);

- Offre d'un minimum de plages horaires de vaccination contre l'influenza durant toute la période recommandée;

- Vaccination contre l'influenza dans les milieux de vie;

- Rétroaction des couvertures vaccinales aux vaccinateurs en milieu scolaire;

- Offre de la vaccination contre le pneumocoque au moment de la vaccination contre l'influenza;

- Maintien de la qualité du Protocole d'immunisation du Québec et diffusion à tous les vaccinateurs;

- Ajout d'un indicateur de gestion : vaccination contre l'influenza chez les travailleurs de la santé;

- Développement de formations en ligne;

- Mise sur pied d'un groupe de travail sur la formation en immunisation.

CONCLUSION : Les experts ont apprécié le processus systématique utilisé. Afin d'évaluer la faisabilité et l'acceptabilité des interventions identifiées, une consultation auprès des autorités de santé publique à l'échelle régionale et des vaccinateurs sera réalisée.

\section{P034}

ROTAVIRUS VACCINATION: RESULTS OF A LONGITUDINAL STUDY ON DETERMINANTS OF CANADIAN PARENTS' DECISION

E Dubé $^{1}$, JA Bettinger ${ }^{2}$, B Halperin ${ }^{3}$, R Bradet ${ }^{1}$, F Lavoie ${ }^{1}$,

C Sauvageau ${ }^{1}, V_{\text {Gilca }}{ }^{1}$, N Boulianne ${ }^{1}$

${ }^{1}$ Quebec, QC; ${ }^{2}$ Vancouver, BC; ${ }^{3}$ Halifax, NS

BACKGROUND AND AIMS: Rotavirus disease is a common cause of health care utilization and almost all children are affected by the age of 5. In the context where rotavirus immunization is recommended, but not publicly-funded, the main objective of this study was to examine the determinants of parent's acceptance to have their child immunized.

METHODS: The survey instruments were based upon the theories of planned behavior and interpersonal behavior. Data were collected by telephone interviews. Main outcome measures were (1) parents' intention to have their child immunized against rotavirus (phase I) and (2) the children's immunization status (phase II).

RESULTS: As of April 6 2010, 408 and 358 parents have completed respectively Phase I and II. Most parents (67\%) intended to immunize their child against rotavirus. Factors significantly associated with parental intentions were: perceptions of the moral correctness of having their child immunized (partial $\mathrm{R}^{2}=0.64, \mathrm{p}<0.0001$ ); perceptions that significant others will approve the immunization behaviour (partial $\mathrm{R}^{2}=0.06, \mathrm{p}<0.0001$ ); household revenue (partial $\mathrm{R}^{2}=0.01, \mathrm{p}=0.0003$ ) and perceived capability of having their child immunized (partial $\left.\mathrm{R}^{2}=0.01, \mathrm{p}<0.0001\right)$. In Phase II, 161 parents $(45 \%)$ reported that their child was immunized against rotavirus. The cost of the rotavirus vaccine and the fact that the vaccine will not protect the child against all diarrhea were the main barriers perceived by parents who decide not to vaccinate their child

CONCLUSION: Surveyed parents generally held positive attitudes toward rotavirus vaccines. Most parents with strong intentions to have their child immunized effectively did it. The cost of the vaccine is an important barrier to rotavirus vaccination.

\section{P035}

FACTORS INFLUENCING PAIN MANAGEMENT

DURING ROUTINE SCHOOL-AGE AND ADOLESCENT IMMUNIZATIONS: FINDINGS FROM A FOCUS-GROUP PILOT STUDY WITH NURSES WORKING IN PUBLIC HEALTH

\author{
A Kikuta ${ }^{1}$, F Gardezi ${ }^{2}$, V Dubey ${ }^{1}$, A Taddio ${ }^{1}$ \\ ${ }^{1}$ Toronto, ON; ${ }^{2}$ Ottawa, ON
}

BACKGROUND: Despite the availability of a variety of evidencebased interventions, it has previously been reported that the majority of infants and children undergo vaccine injections without the benefit of analgesia. Nurses in public health administer a substantial number of injections. Their attitudes and practices, however, surrounding acute pain during vaccine injections have not been previously explored.

PURPOSE: To determine the child immunization pain and pain management perspectives of nurses working in public health as well as identify unique barriers to their immunization settings.

METHODS: A pilot focus group was conducted with 10 nurses that immunize children in the city of Toronto in school and community clinic settings. Participants reported their attitudes and practices with regards to vaccine injection pain and pain management.

RESULTS: Three key themes emerged: environmental and process factors, perceptions about the effectiveness of different analgesic interventions, and perceptions about the importance of acute pain. Participants reported they felt there was a lack of control over their environment, resulting in fear and discomfort for children. They recommended increased support from external partners, such as school teachers and administrators. Participants reported that pharmacological interventions, such as topical local anaesthetics, were not used, but that psychological interventions, such as distraction, were commonly used. Nurses questioned the effectiveness of topical anaesthetics and their role. Overall, nurses reported that children were primarily concerned about needle pain while they were primarily concerned about children's fear.

CONCLUSION: Nurses reported vaccination setting, analgesic effectiveness, and relative importance given to pain as important factors for pain and pain management during vaccine injections. Future studies should explore the views of nurses in public health in other geographical regions. Efficacy of educational resources and programs targeted to these nurses and their external partners, including parents, should be investigated.

\section{P036}

MUMPS IN PRISON: DESCRIPTION OF AN OUTBREAK IN MANITOBA, CANADA

A Walkty ${ }^{1}$, B Fatoye $^{2}$

${ }^{1}$ Winnipeg, MB; ${ }^{2}$ Beausejour, MB

BACKGROUND: Prisoners may represent a group of individuals at risk for mumps infection, related to close living conditions and inadequate vaccination. There is a lack of published information on the management of mumps in a prison setting. 
PURPOSE: The purpose of this report was to describe an outbreak of mumps that occurred in a medium security correctional centre (Milner Ridge) in Manitoba, Canada.

METHODS: A case definition of mumps consistent with that in the document "Guidelines for the Prevention and Control of Mumps Outbreaks in Canada" was adopted. Cell culture, polymerase chain reaction, and serology were used for case confirmation.

RESULTS: Five confirmed cases of mumps infection were identified at the Milner Ridge Correctional Centre between Jan. 12 and Feb. 5, 2009. One additional confirmed case and 3 additional probable cases were identified at a second correctional centre. Outbreak control at Milner Ridge was accomplished by cohorting the affected units of the centre, providing inmates and staff with information on infection control measures, deferring transfers, notifying other provincial correctional centres, federal prisons and physicians in Manitoba of the outbreak, and performing active surveillance for new cases. Vaccination was offered to all inmates and staff at Milner Ridge on the assumption, based on average inmate age, that the majority of these individuals would have previously received, at most, a single dose of mumps containing vaccine (suboptimal protection).

CONCLUSION: An outbreak of mumps in a correctional setting was successfully contained via implementation and tailoring of basic infection control measures, and vaccination of inmates and staff. Given the relatively young age of many prisoners and the parallels between prisons and dormitories, it could be argued that prisoners may represent another group of individuals for whom a second dose of mumps vaccine (if not received in childhood) would be beneficial as primary prevention.

\section{P037}

\section{OUTBREAK SUMMARIES: A TOOL FOR MONITORING TRENDS IN VACCINE PREVENTABLE, RESPIRATORY AND ENTERIC INFECTIOUS DISEASE OUTBREAKS}

\section{Helferty, J Pulickal, V Keegan \\ Ottawa, ON}

BACKGROUND: Outbreak Summaries provides a national secure, web-based communication system for summarizing vaccine preventable, respiratory and enteric disease outbreak information in a systematic and standardized manner. Outbreak Summaries is the first tool that will allow provinces and territories to summarize and report on characteristics of outbreaks, monitor trends in order to impact program, vaccine policy and direct future investigations. Public health professionals involved in this application will have the ability to document, query, summarize and generate reports on outbreaks within their jurisdiction and in others to compare disease agents, severity of disease and other epidemiological factors.

PURPOSE: To promote and utilize an online application that will allow provinces and territories to systematically summarize and extract respiratory and vaccine-preventable outbreak data including attack rates, immunization rates, public health interventions which were captured during the outbreak investigation.

METHOD: Through discussions and presentations, the Public Health Agency of Canada has every intention to continue to implement Outbreak Summaries in provinces and territories.

RESULTS: The Vaccine-preventable disease and respiratory module of Outbreak Summaries went live in Nova Scotia in 2009. To date greater than 30 summaries have been posted since June 2010 and uploaded historical data from 1997-2009 has also been posted. The Public Health Agency of Canada also launched within the Center for Immunization and Respiratory Infectious Diseases and the Center for Food-borne, Environmental and Zoonotic Infectious Diseases. Discussions are underway with other provinces and territories to launch the application

CONCLUSION: Outbreak Summaries has been well received in Nova Scotia and it is hoped the application will be launched in several additional provinces/territories in 2010. This application will enhance national outbreak data quality and reliability by ensuring clear evidence is captured for each outbreak event. These data will be useful to public health and healthy public policy professionals at all levels of government for identification of risk factors and informing policy aimed at preventing respiratory and vaccine-preventable outbreaks through immunization.

\section{P038}

DOCUMENTATION OF THE TIME INVOLVED IN VACCINE INJECTION APPOINTMENTS IN CHILDREN: IS THE ADDITION OF ANALGESIC INTERVENTIONS FEASIBLE? A Taddio, M Hogan, S Gerges, A Girgis, P Moyer, L Wang, T Ho, E Colledge, $S$ Greenberg, $M$ Ipp Toronto, ON

BACKGROUND: Pain from vaccine injections remains undertreated, despite the availability of numerous analgesic strategies. Clinicians report that one of the reasons for sub-optimal analgesic uptake is a lack of time to incorporate them within current office workflows. To date, no study has documented the amount of time that is available to educate parents regarding administration of analgesics to children undergoing vaccine injections.

PURPOSE: To document the time involved in vaccine administration and to determine the feasibility of offering education and analgesic strategies for immunization pain management.

METHODS: Four urban outpatient primary care practice settings (three paediatric and one family practice) in Toronto were included. For 50 consecutive immunization appointments in children at each site (200 in total), trained research assistants recorded: clinic arrival time, time entering into examination room, and time of first vaccine injection. The primary outcome of interest was total time between clinic arrival and the first vaccine injection.

RESULTS: The median (range) age of children undergoing vaccine injections was 15 months (1-204). The mean (SD) time between clinic arrival and the first vaccine injection was 34 minutes (17), with a range of $7-93$ minutes. There was a significant $(\mathrm{p}<0.05)$ difference among participating practice settings, with the mean waiting time ranging from 21 minutes (11) to 47 minutes (17). Most of the time was accounted for by time spent in an examination room.

CONCLUSION: The results demonstrate that ample time is available to provide educational materials and analgesics to mitigate pain during vaccine injection appointments in children. Additional research is needed to determine the interventions that are most effective within the time constraints of specific practice settings. The impact of analgesic interventions on vaccination appointment duration and parental and immunizer satisfaction with the immunization experience also warrant investigation.

\section{P039}

\section{IS GLUCOSE A SWEET SOLUTION FOR REDUCING IMMUNIZATION PAIN? RESULTS OF A SYSTEMATIC REVIEW AND META-ANALYSIS}

\section{Hogan, L Wang, V Shah, A Taddio Toronto, ON}

BACKGROUND: Sweet solutions such as glucose and sucrose are recommended for procedural pain in infants. Sucrose solution is not widely commercially available and therefore glucose solution may represent a more feasible alternative because it is generally available in medical settings. To date there has been no systematic review and meta-analysis of its effect on immunization pain in infants and a critical review and synthesis of the data is required.

PURPOSE: To determine the effectiveness of oral glucose for pain reduction in infants undergoing immunization injections.

METHODS: The following databases were searched: MEDLINE (1950-June 2010), EMBASE (1980-June 2010), CINAHL (1982-June 2010) and Cochrane Library (June 2010) using key words: glucose, pain, 
immunization. References from review articles were also checked. Inclusion criteria were randomized or quasi-randomized trial, preterm and term infants up to 1 year of age undergoing immunization, treatment group of glucose solution and control group of placebo water. Data were extracted onto pre-designed forms. Quality was assessed using the Cochrane risk of bias tool. The primary outcome was pain as assessed by validated pain scales or cry duration. When data could be combined, standardized mean differences (SMD) were calculated as measures of effect along with $95 \%$ confidence intervals (CI) using a fixed-effects model.

RESULTS: Four studies with 908 infants were identified. Vaccines administered included hepatitis $B(n=2)$ and diphtheria, tetanus, pertussis, inactivated poliovirus, Haemophilus influenzae type B vaccine $(\mathrm{n}=2)$. The amount of glucose administered ranged from $0.25 \mathrm{~g}$ to $1 \mathrm{~g}$. Glucose significantly reduced pain compared to placebo water (SMD $-0.30[95 \% \mathrm{CI}-0.40,-0.20])$. One study had a low risk of bias, two had an unclear risk of bias and one had a high risk of bias (insufficient concealment of allocation).

CONCLUSION: Glucose reduces pain from immunization injections in infants. The magnitude of effect should be compared with sucrose solution.

\section{P040 \\ RELIABILITY AND VALIDITY OF THREE SCALES FOR PAIN ASSESSMENT IN INFANTS UNDERGOING IMMUNIZATION INJECTIONS}

\section{A Taddio, M Hogan, P Moyer, A Girgis, M Ipp, D Stephens Toronto, ON}

BACKGROUND: At present, there are few data evaluating the psychometric properties of scales used to assess acute pain following immunization injections in infants. Reliable and valid pain assessment scales are necessary to evaluate the effectiveness of pain management interventions.

PURPOSE: To evaluate the psychometric properties of 3 measures of acute pain in infants undergoing immunization injections.

METHODS: 120 infants aged 2-6 months participated in a doubleblind, randomized clinical trial whereby the pain caused by two different vaccines [diphtheria, polio, and tetanus toxoids and acellular pertussis and Haemophilus influenzae type b (DPTaP-Hib) and pneumococcal conjugate vaccine (PCV)] was compared. Pre-injection (baseline) and post-injection (vaccine) pain assessments were made by blinded research assistants from videotaped recordings of the procedure using the Modified Behavioural Pain Scale (MBPS), Neonatal Infant Pain Scale (NIPS), and the Faces Legs Activity Cry Consolability (FLACC). Reliability was assessed by comparing baseline and vaccine scores between raters. Internal consistency was assessed using Cronbach's alpha. Construct validity was determined by assessing: the responsiveness to vaccine injection; responsiveness to vaccine type, and between-scale correlations.

RESULTS: Baseline and vaccine reliability coefficients for MBPS, NIPS and FLACC were all greater than 0.85. For all measures, Cronbach's alpha ranged from 0.83 to 0.94 . Vaccine scores were higher $(\mathrm{p}<0.05)$ than baseline scores for MBPS (2.0 vs. 7.2), NIPS (0.3 vs. 5.3), and FLACC (0.6 vs. 6.5$)$. Vaccine scores were also higher $(\mathrm{p}<0.05)$ for PCV when compared to DPTaP-Hib, as assessed by the MBPS (6.3 vs. 8.2 ), NIPS (4.4 vs. 6.2), and FLACC (5.3 vs. 7.8). Between-scale correlation coefficients ranged from 0.84 to 0.92 .

CONCLUSION: Reliability and construct validity were demonstrated for the MBPS, NIPS and FLACC. Further evaluation of the feasibility and clinical utility of these measures are recommended to determine the most appropriate fit for different clinical settings.
P041

MAXIMIZING SCOPE OF PRACTICE IN IMMUNIZATION
PROGRAM DELIVERY: AN EVALUATION OF A
COLLABORATIVE PRACTICE MODEL FOR A
SCHOOL-BASED IMMUNIZATION PROGRAM IN A LARGE
URBAN HEALTH UNIT IN NOVA SCOTIA

NW Whelan, L Humber

Dartmouth, NS

BACKGROUND: A number of reports have highlighted the need to make better use of increasingly scarce health human resources, and address the underutilization of such resources in working to their full scope of practice.

A collaborative practice model for immunization program delivery was implemented in September 2008 with a coordinating role delivered by an Immunization Team Lead with five Licensed Practical Nurses (LPNs). All Public Health Nurses (PHNs) completed an immunization competency program and a minimum of two clinic days over the course of the school year.

PURPOSE: The objectives of the project were to evaluate the impact of the model on competency for immunization and pandemic preparedness, assess the effectiveness of the model in maximizing scopes of practice for RNs and LPNs across Public Health programs, and evaluate the economic impact.

METHODS: An online questionnaire was distributed to all nurses working within the public health unit $(n=76)$ in the spring 2009 through survey tool selectsurvey.net. with a $61 \%$ response rate $(n=46)$.

The cost analysis compared the staffing costs of scheduled clinics throughout the school-year to the previous school-year (pre-post).

RESULTS: $94 \%$ of respondents strongly agreed/agreed that they felt prepared and confident to participate in mass immunization clinics, including pandemic response. $84 \%$ clearly understood their role in the collaborative practice model. A key theme of the qualitative data suggests that the model allowed a focus on maximizing scope of practice for RNs in other program areas, in particular, the health promoting school model, "the collaborative practice model enabled me to attend fewer clinics, thus enabling me to enhance my work in other areas."

There was a $41 \%$ cost reduction in staffing costs per student for the Grade 7 program and a $44 \%$ cost reduction for the Grade 10 program from 2007-2008 to 2008-2009.

CONCLUSION: The implementation of the model was successful in year one of the program; some areas of improvement were highlighted for program strengthening. A collaborative model ensures the appropriate utilization of resources, as well as building capacity for pandemic preparedness.

\section{P042}

\section{ATTITUDES AND BELIEFS OF HUTTERITE MOTHERS TOWARD EARLY CHILDHOOD IMMUNIZATION IN THE ASSINIBOINE HEALTH REGION IN MANITOBA, CANADA}

\section{Johnson}

Boissevain, MB

BACKGROUND: Hutterites are a growing population of communal living Christians, with over 350 colonies in western Canada. Their preschoolers can have lower rates of completed immunizations (per routine schedule) than non-Hutterites. The importance of immunization to child health is proven, therefore it is important to examine how Hutterite beliefs could affect immunizing practices.

OBJECTIVE: To explore attitudes and beliefs of Hutterite mothers in the Assiniboine Health Region (SW Manitoba) related to immunization of their preschool children.

METHODS: Qualitative research used semi-structured interviews of 20 Hutterite mothers of preschoolers, within the Assiniboine Health Region. Participants were obtained through purposive, convenience and snowball sampling, with data analyzed thematically. 
RESULTS: Immunization knowledge was limited and included beliefs of undesirable reactions. Most participants believed that health care professionals, colony and family members were trustworthy, reliable information sources, whereas government and pharmaceutical companies were held to be untrustworthy. Hutterite mothers expressed that religion, colony life, patriarchal focus, and family influenced immunization decisions. Seeing colony life as protective from disease was a pervasive view, influencing some to decide against immunizations. Many participants articulated preferences of delaying babies' first immunizations, disapproved of multiple immunizations, postponed or cancelled if children seemed unhealthy or for colony duties. H1N1 immunization was viewed very negatively by most; often reported as a moneymaking scheme, unnecessary or dangerous.

CONCLUSION: Inaccurate knowledge may have originated from limited access to information channels and low education levels, with immunization "stories" spreading easily within close colony connections. Trusted relationships with health care professionals could be an opportunity to discuss immunization concerns and provide accurate information. Hutterite culture, including the husband's prominent role, affects decision-making. Therefore increasing health care professionals' cultural understanding may assist in lessening immunization deterrents. The 2009 H1N1 immunization campaign highlighted the need for different information channels and methods to encourage immunization among this population.

\section{P043}

"EVERGREEN" CANADIAN IMMUNIZATION GUIDE: PROVIDING ACCURATE INFORMATION EFFICIENTLY TO IMMUNIZATION STAKEHOLDERS

SJ Ismail1,2,3 , P Varughese ${ }^{1}$, N Klassen ${ }^{1}$, G Badger ${ }^{1}$, JM Langley ${ }^{4}$, B Warshawsky ${ }^{5}$, T Harris ${ }^{1}$, M FarhangMehr ${ }^{1}$, D Elliott ${ }^{1}$, J Lynch ${ }^{1}$,

E Russell ${ }^{1}$, J Laroche ${ }^{1}, \mathrm{~V}$ Wilson ${ }^{1}$

${ }^{1}$ Ottawa, ON; ${ }^{2}$ Edmonton, AB; ${ }^{3}$ Calgary, AB; ${ }^{4}$ Halifax, NS;

${ }^{5}$ London, ON

BACKGROUND: The Canadian Immunization Guide (CIG) is relied upon in the field by immunization providers and policy and program planners as the primary source of Canadian immunization information. Currently the CIG is not regularly updated with new evidence or NACI recommendations, so an "evergreen" web-based guide has been endorsed by the National Advisory Committee on Immunization (NACI) to reduce gaps in knowledge translation. The United Kingdom ("Green Book") and the United States ("Red Book") have implemented similar web-based immunization guides.

PURPOSE: The goal of the evergreen CIG is to produce an up-todate, web-based, user-friendly guide to provide accurate information to immunization stakeholders in a timely, accessible, efficient and effective manner.

METHODS: The process to attain the goal involves a consultant (to assist with writing/editing), a steering committee (including immunization, communication, website experts), the NACI Executive Committee (in an advisory role), and chapter working groups (comprised of NACI members, external subject experts, Canadian Immunization Committee and Committee on Tropical Medicine And Travel health representatives as appropriate).

RESULTS: The Internet is increasingly used by immunization stakeholders. The HTML CIG version is among the top most visited Public Health Agency of Canada websites, and the number of hits has increased since 2006. The National Physician Survey estimates that most physicians have Internet in their "main patient care" setting and this proportion is increasing ( $71 \%$ in $2004 ; 80 \%$ in 2007). A user-friendly, expedient process for the dissemination of online updates to CIG users will be developed, and options to accommodate those stakeholders who are averse to a web-based guide or who do not have consistent access to the Internet are being explored.

CONCLUSIONS: Work on the evergreen CIG has begun. Phase 1 of the project will be to update Part 4 of the CIG ("Active Immunizing
Agents"). A targeted, stakeholder communications strategy will be developed and evaluations to assess and address issues such as user satisfaction and ease of accessibility will be ongoing.

\section{P044}

"TAKE YOUR BEST SHOT": EVALUATION OF A VIDEO RESOURCE FOR TORONTO PUBLIC HEALTH'S SCHOOL-BASED IMMUNIZATION PROGRAM

\section{J Kaashoek, A Mathur, J Cameron, V Dubey Toronto, ON}

BACKGROUND: Toronto Public Health (TPH) annually offers grade 7 students hepatitis $\mathrm{B}$ and meningococcal vaccines in school immunization clinics. In 2009, TPH developed an educational video called "Take Your Best Shot" in response to teachers' requests for a resource to provide immunization information to students. The video was available as a DVD and on-line. In September 2009, 449 Toronto schools received the "Take Your Best Shot" DVD.

PURPOSE: To evaluate the usefulness of the video by grade 7 teachers at Toronto schools.

METHODS: In September and October 2009, a feedback survey was administered to grade 7 teachers who accompanied their class to the first dose hepatitis B-meningococcal vaccination clinics.

RESULTS: A total of 115 surveys from 63 schools were analyzed. 58\% of teachers (67 out of 115) reported knowing about the video and of these $52 \%$ (35 of 67) watched the video with their class. Of teachers who watched the video, $57 \%$ (20 of 35) reported it was "very helpful"; $45 \%$ (16 of the 35 teachers) reported it was "somewhat helpful". $41 \%$ (48 of 115) of teachers reported not knowing about the video and of these, $77 \%$ indicated they wanted to show the resource to students before the second scheduled immunization clinic. The most common way that teachers found out about the video was through contact with the school secretary and a teacher's checklist tool that was part of the student vaccine consent package.

CONCLUSION: The "Take Your Best Shot" video was very helpful to the majority of teachers who watched the video with their class. Implementing a feedback survey was helpful in raising awareness about the video and to gain additional feedback. Developing video resources are an effective way to communicate important immunization information in schools.

\section{P045}

\section{COLD CHAIN DISTRIBUTION STRATEGIES IMPLEMENTED TO OVERCOME UNIQUE CHALLENGES IN RURAL COMMUNITIES}

$\underline{\text { SC Keefe }}{ }^{1}$, HD Cooze ${ }^{2}$

${ }^{1}$ Goose Bay, NL; ${ }^{2}$ Gander, NL

BACKGROUND: In early 2009, with the imminent threat of Pandemic (H1N1) 2009, regional health authorities in Newfoundland and Labrador recognized the delivery challenges inherent in transporting large volumes of vaccine to rural communities. An existing cold chain distribution system was previously established; it was recognized that enhancements were needed.

PURPOSE: This delivery system was developed to implement a systematic approach in order to reduce vaccine wastage, thus protecting vaccine stability.

Provincial guidelines for transportation and storage of biological products (from the National Storage and Handling Guidelines from PHAC) were already adopted throughout the province. However, challenges existed that were unique to rural Newfoundland and Labrador communities, including: geography, communication, timeliness of vaccine distribution, and extremes in weather. Regional health authorities recognized these challenges and developed strategies to mitigate the barriers.

METHODS/PROJECTS: The Communicable Disease Control Nurse, in consultation with our vaccination distribution partners, 
evaluated all critical contact points during vaccine shipment where breaks in cold chain were a potential problem. The contact points or "check points" were identified from the onset of vaccine distribution through to arrival at the district office. Five sequential check points, key to safe vaccine delivery, were identified during the assessment: vaccine coordinator (initial packaging), ground courier, air/sea courier, vaccine recipient, and vaccine coordinator (assessment).

RESULTS: Improvements to cold chain distribution have been employed for less than one year; a formal evaluative report is not currently available. During Pandemic (H1N1) 2009, anecdotal evidence suggests minimal vaccine wastage occurred during shipment. Evaluation and process enhancements resulted in the implementation of two strategies to assist with cold chain break monitoring: an informal evaluation process (incident report form), and a vaccine cold chain break tracking tool.

CONCLUSION: A systematic approach, involving the key sequential check points during vaccine shipment, resulted in a successful distribution of stable vaccine to the entire population of Newfoundland and Labrador.

\section{P046}

\section{USING WEB 2.0 TECHNOLOGIES TO UNDERSTAND PUBLIC CONCERNS AND PROVIDE INFORMATION}

\section{A Kitta ${ }^{1,2}$ \\ ${ }^{1}$ Greenville, NC; ${ }^{2}$ St John's, NL}

BACKGROUND: Web 2.0 technologies are one of the most commonly used forms of communication by the public, but they are largely overlooked by researchers and institutions.

PURPOSE: To study how often vaccines issues are mentioned on Facebook by searching the entire site for vaccine and H1N1 related keywords.

METHODS: Searches were performed during the height of the H1N1 crisis, after major news stories concerning vaccines, and at random to see how often vaccines were mentioned and what was being said about them.

RESULTS: As expected, vaccine discussions were highest during peak periods, such as flu season and after pertinent news coverage. Discussions were overwhelmingly anti-vaccination and often resulted in response to Facebook status update. Additionally, some vaccine institutions used Facebook with great success to promote availability of vaccines.

CONCLUSION: Web 2.0 technologies, such as Facebook, can provide access to the more recent and pertinent concerns of the public and potentially address those issues. Additionally, institutions can promote their own research, initiatives, and availability to the public at little to no cost.

\section{P047 \\ THE ROLE OF INDIVIDUAL CASE ASSESSMENT IN EVALUATING THE SAFETY OF VACCINES - OUTCOMES FROM THE CANADIAN ADVERSE EVENTS FOLLOWING IMMUNIZATION (AEFI) SURVEILLANCE SYSTEM (CAEFISS) \\ $\underline{\text { R Pless, }}$ T Freeland, J Lafleche, B Law \\ Ottawa, ON \\ BACKGROUND: The cornerstone of vaccine safety surveillance is the reporting of adverse events by health care providers. However, case reports may be incomplete or may describe serious or unusual events of unclear relationship to vaccination that may require specialist review to help assess. Typically, conclusions of this assessment are defined on a World Health Organization (WHO) scale from related to unrelated, but are sometimes unassessible if there is insufficient information. \\ PURPOSE: To describe the experience with the assessment of cases over a 15 year period, conducted with cases from the Canadian adverse event reporting system. The outcome of case assessments and implications}

for the surveillance of safety is reviewed in light of some concerns with the validity of the WHO scale.

METHODS: Cases that have been selected for or undergone individual case assessment were extracted from the AEFI database. Details of the cases and their assessment were analyzed.

RESULTS: From 1994-2009, 937 cases were reviewed by an expert committee. The most frequent reasons for selection for review included hospitalization over 3 days, thrombocytopenia, seizure, death, anaesthesia/paraesthesia, GBS, encephalitis and anaphylaxis. Assessment of the association with vaccination were: $11 \%$ very likely, $23 \%$ probable, $19 \%$ possible, $19 \%$ unlikely and $23 \%$ unrelated, while $4 \%$ could not be assessed with the information provided. Reviewers felt, however, that the scoring system was biased towards deeming a relationship possible solely on the basis of not having an alternate explanation and a timing that follows vaccination.

CONCLUSIONS: While on the surface, it appears feasible to ascertain the association between a vaccine and an AEFI at the individual, the exercise is fraught with caveats. More appropriately, expert case reviews serve to ensure that no signal is missed on the basis of insufficient information or a plausible new entity, given the specialist resources that are brought to bear on the evaluation of each case, rather than providing a definitive conclusion for any individual case.

\section{P048}

\section{THE EFFECTIVENESS OF AN INTERVENTION TO IMPROVE MMR IMMUNIZATION COVERAGE RATES IN CHILDREN}

\section{Rajakumar, M Lemstra, A Thompson}

\section{Saskatoon, SK}

INTRODUCTION: In Saskatoon Health Region (SHR), only $67.4 \%$ of children overall are fully immunized for measles/mumps/ rubella (MMR) at twenty-four months of age with only $43.7 \%$ of low income children fully immunized.

METHODS: The study: a) contacted parents of children who were behind in MMR immunizations to determine knowledge, beliefs and barriers toward immunization; b) determined the effectiveness of a telephone reminder system in improving immunization rates in a health region in comparison to a control health region and c) compared the effectiveness of telephone reminders to telephone reminders combined with home visits in improving child immunization coverage rates in low income neighborhoods.

RESULTS: The survey was completed by 629 parents (69\% response rate). Of those, $81.8 \%$ were not aware their child was behind in immunizations.

In SHR, the MMR immunization coverage increased from $67.4 \%$ to $74.0 \%$ in the first year of intervention ( $R R=1.10 ; 95 \%$ CI 1.08-1.12). All four neighborhood groupings (three urban by income and one rural) had relative increases ranging from nine percent to eleven percent. The control health region observed an immunization coverage increase from $66.5 \%$ to $69.2 \%$ in the first year ( $R R=1.04 ; 95 \%$ CI $1.01-1.07)$.

The three low income neighborhoods with only telephone reminders had an immunization coverage rate of $48.7 \%$ (95\% CI 39.5-57.8). The three low income neighborhoods that received a telephone reminder and home visit had an immunization coverage rate of $60.5 \%$ (95\% CI 52.5-68.6).

CONCLUSION: Telephone reminder systems have some benefit in increasing child immunization coverage rates. 
P049

ENFORCING IMMUNIZATION LEGISLATION IN PEEL REGION: PROCESSES AND IMPACTS ON COVERAGE RATES, VACCINE UPTAKE AND PUBLIC HEALTH DECISION MAKING

\section{Rowan, A Palmer, I Mogck}

Brampton, ON

BACKGROUND: Prior to 2006 student immunization legislation enforcement in Peel region was deferred due to funding shortfalls. In 2006 a review of the available data indicated that coverage rates in Peel had dropped as low as $60 \%$, well below the $95 \%$ coverage target. As a result a large scale project was undertaken to mitigate the risk of apparent low immunization rates among students in Peel to control the spread of infectious diseases.

PURPOSE: The objectives of the project were to achieve a $95 \%$ coverage rate among Peel students for antigens required under the Immunization of School Pupils Act; and to have updated immunization information for Peel students to support effective public health decision making.

METHODS: A project was implemented to screen and update immunization records, and enforce the requirements for all of Peel's approximately 250,000 students. A subsequent measles outbreak in Southern Ontario highlighted the importance of the project which was accelerated with a goal to complete one year earlier than originally planned. The implementation and evaluation of this project provided important information about the impact of legislation.

RESULTS: Progressive enforcement of the immunization legislation resulted in immunization coverage rates increasing from $47.7 \%$ to $90.3 \%$ for diphtheria, tetanus and polio (DTP) and from $68.5 \%$ to 93.7\% for measles, mumps, rubella (MMR). More than 44,000 doses of vaccine were administered. Between 14.8\% (MMR) and $24.2 \%$ (DTP) of students screened had been appropriately immunized before the strategy; however their immunizations were not reported to Public Health.

CONCLUSION: Legislation is a powerful tool for achieving and maintaining high vaccine coverage rates. It helps to engage stakeholders, creates a call to action to vaccinate beyond infancy and ensures data needed for Public Health decision making is accurate; large, complex stakeholder systems create significant challenges for implementation; and a large project-based approach facilitates success.

\section{P050}

\section{AN INTERACTIVE TOOLKIT FOR PUBLIC HEALTH NURSES TO EDUCATE YOUNG ADOLESCENT GIRLS ABOUT HUMAN PAPILLOMAVIRUS, CERVICAL CANCER AND THE GARDASIL ${ }^{\circledR}$ VACCINE}

GJ Stagg-Sturge

\section{St John's, NL}

BACKGROUND: Human Papillomavirus (HPV) is the most common sexually transmitted infection in Canada and has the potential to cause cervical cancer. Overall, there is a lack of knowledge about HPV and cervical cancer in early adolescence, and specific primary prevention strategies need to be developed and implemented specifically for teaching this population.

PURPOSE: The objective of this project was to develop a facilitator's toolkit to guide public health nurses in teaching grade six girls about human papillomavirus, cervical cancer, and the gardasil vaccination. METHODS: Key informant interviews were conducted with stakeholders to assess the need for a facilitator's toolkit for nurses. A literature review was completed and a focus group was held with the target aggregate in a community centre setting to assess knowledge levels pertaining to human papillomavirus, cervical cancer, and the gardasil vaccination. A toolkit was developed for grade six girls that is based on the appropriate developmental level.
RESULTS: It was determined that there is a lack of a formal standardized teaching tool that is currently being utilized with the grade six population, in conjunction with the HPV immunization program in Newfoundland and Labrador. A lack of knowledge was established throughout the focus group and within the literature.

CONCLUSION: There is currently little health promotion tailored specifically to girls in grade six related to HPV/ HPV vaccination and there is a need to have more community based health programs, targeted at young adolescents to supplement what is being taught in schools. The development and implementation of this comprehensive facilitator's toolkit will work towards meeting the identified need and nurses who work with community centers/schools can deliver. It will ultimately increase the girls' level of knowledge regarding the HPV virus and how it is spread, and will also facilitate the realization that there are key actions young adolescents can take to prevent cervical cancer. This knowledge can potentially increase conversations at home with parents regarding sexual health, HPV and the Gardasil® vaccine, which can lead to increased vaccine uptake.

\section{P051}

\section{SURVEY OF ATTITUDES AND PRACTICES OF PHYSICIANS AND NURSES REGARDING ANALGESIA DURING ROUTINE CHILDHOOD IMMUNIZATIONS}

\section{A Kikuta, H Boon, V Shah, J Stinson, A Taddio Toronto, ON}

BACKGROUND: Parents report that pain during vaccine injections is the prominent feature of the immunization experience and that they look to their healthcare provider for information about how to mitigate vaccine injection pain. To date, there have been no studies that have examined healthcare providers' views of pain and pain management during childhood immunization and the practices and factors that hinder or facilitate optimal pain management.

PURPOSE: To determine healthcare providers' perspectives about pain and pain management during childhood immunization.

METHODS: A descriptive qualitative study methodology employing a naturalistic paradigm was utilized. Semi-structured interviews were conducted with 25 healthcare providers (doctors and nurses) that routinely administer vaccines in children in hospital and family practice settings. Participants reported their attitudes and practices with regards to vaccine injection pain and pain management. The semi-structured interview guide evolved to address specific knowledge deficits highlighted in prior interviews. Interviews were transcribed and coded using NVivo qualitative analysis software. Qualitative content analysis was used to identify major themes.

RESULTS: Three major themes were identified: attitudes towards pain and pain management, feasibility of analgesic interventions, and analgesic intervention efficacy. Immunization pain was reported as relatively unimportant by the majority of healthcare professionals; instead, fear/anxiety was identified as the most concerning aspect of vaccine injections. All participants reported using pain-relieving strategies regarded as 'feasible', including: psychological (e.g., distraction) and physical (e.g., parental holding) interventions. Participants disregarded pharmacological (e.g., topical anesthetics) strategies, mostly due to uncertainty about efficacy and implementation within current office workflows.

CONCLUSION: Efforts to improve pain management during vaccine injections in children should aim to address the potential for optimal pain management to reduce the development of fear, and the feasibility and impact of better pain management practices, including the use of topical anesthetics. 
P052

COCCOONING STRATEGY IN RESPONSE TO AN INCREASE IN INFANTILE PERTUSSIS

R Tuchscherer, H Bangura, L van Haarlem, S Shahab, B Adhikari, L Sly

Regina, SK

BACKGROUND: Unlike other vaccine preventable childhood diseases pertussis remains endemic in the Canadian population. While pertussis presents as a mild cough in most adults vaccinated in childhood, it can lead to hospitalisation and death in infants.

PURPOSE: A strategy is presented that addresses the need to protect infants too young to be protected by vaccination.

METHODS: All cases of infantile pertussis reported to the province over a three year period were reviewed, including age at onset of pertussis, vaccination history and history of contact with a known or suspected case of pertussis.

RESULTS: The epidemiology of infantile pertussis was analysed. Saskatchewan saw an increase in infantile pertussis from a low of 34 cases per 100,000 in 2005 to $117 / 100,000$ in 2009 . $43 \%(15 / 35)$ of cases reported from 2008 to April 2010 were in infants under two months of age and hence too young to be protected by immunization. There were five deaths in infants during this period, all of which were in unvaccinated infants under 3 months of age. Only $6 \%(2 / 35)$ of cases were in infants fully up to date with immunization. The remainder $51 \%$ of cases (18/35) were in infants older than 2 months but not up to date for immunization.

CONCLUSION: Pertussis vaccine has good efficacy if given on time. However, infants younger than 2 months of age remain unprotected. A cocooning strategy was proposed including vaccinating mothers and other close care givers at birth. A long term strategy to increase population level immunity through wider adult pertussis booster vaccination is required. Till such time, cocooning infants, who are at highest risk of morbidity and mortality from pertussis, may be an effective short term measure especially when faced with rising incidence of infantile pertussis.

\section{LABORATORY VACCINOLOGY SCIENCE}

\section{P053}

\section{A MENINGOCOCCAL ANTIGEN TYPING SYSTEM (MATS) FOR ESTIMATING THE POTENTIAL EFFECTIVENESS OF PROTEIN-BASED VACCINES AGAINST NEISSERIA MENINGITIDIS}

J Donnelly ${ }^{1}$, D Medini ${ }^{1}$, G Boccadifuoco ${ }^{1}$, A Biolchi ${ }^{1}$, M Stella ${ }^{1}$, S Toti ${ }^{1}$, M Comanducci ${ }^{1}$, A Kleinschmidt ${ }^{2}$, W Andrews ${ }^{3}$,

L De Tora ${ }^{4}$, R Rappuoli ${ }^{1}$, M Giuliani ${ }^{1}$

${ }^{1}$ Siena, Italy; ${ }^{2}$ Marburg, Germany; ${ }^{3}$ Emeryville, CA;

${ }^{4}$ Cambridge, MA

Canadian decision makers will have to decide how best to implement vaccination programs against group B meningococcal disease in their population. Such decisions will be made considering the burden of disease that the candidate vaccines are designed to prevent.

The serum bactericidal assay using human complement (hSBA), is a surrogate marker of protection used in evaluations of meningococcal vaccines. For vaccines based on novel proteins, existing methods may not reflect the variations in sequence and expression levels of the antigens. Testing large panels of strains in the hSBA against immune sera is impractical.

We developed a method, the Meningococcal Antigen Typing System (MATS), to define the potential of diverse MenB strains to be killed by vaccine-induced bactericidal antibodies. MATS is a modular system that may combine both genotypic and phenotypic information depending on the composition of the vaccine to which it is linked. To measure the contributions of $\mathrm{HHBP}, \mathrm{NadA}$, and NHBA, this method uses a novel sandwich ELISA performed on detergent extracts of bacteria. The measurement is sensitive to variations in both the immunologic recognition of each antigen and its expression level. Because the vaccine under study also contains $\mathrm{OMV}$, the contribution of PorA is assessed by conventional subtyping methods.

We correlated MATS results from $124 \mathrm{MenB}$ strains with hSBA data on pooled immune serum from different age groups, and found that a positive MATS result indicates strains that are highly likely to be killed in the hSBA. The method can be performed on large collections of strains, making it possible to survey regional populations of MenB bacteria to determine the potential for strain coverage by a candidate MenB vaccine.

\section{P054}

INTERLABORATORY STANDARDIZATION OF MATS: A PROTEIN ANTIGEN PHENOTYPING SYSTEM FOR N MENINGITIDIS SEROGROUP B

\section{J Donnelly}

\section{Siena, Italy}

The Meningococcal Antigen Typing System (MATS) uses a sandwich ELISA performed on detergent extracts of bacteria to measure the fHbp, NadA, and NHBA content of tested isolates relative to a reference strain (relative potency). MATS relative potency is sensitive to variations in both the immunologic recognition of each antigen and its expression level, and because a vaccine under study also contains OMV, the contribution of PorA is assessed by conventional subtyping methods. In preliminary studies, MATS accurately predicted killing in the serum bactericidal assay using human complement, an accepted correlate of protection for meningococcal vaccines.

We conducted an inter-laboratory standardization study at 7 independent laboratories to assess the robustness of MATS relative potencies for $\mathrm{fHbp}, \mathrm{NadA}$ and NHBA, and to validate the potential for strain coverage in defined geographical regions. Each laboratory performed MATS assays on a set of 18 shared reference strains; each of which was assayed multiple times for the three antigens. 'Consensus' relative potencies were derived for each specimen/antigen combination using a mixed-model analysis of variance from the present data. These values were used to quantify accuracy, reproducibility, and precision among the seven laboratories. We used scatter plots and plots of accuracy, precision, bias and variance components. We calculated Pearson correlation coefficients $(r)$, coefficients of accuracy $(\mathrm{Ca})$, and concordance correlation coefficients (rc), to assess agreement. We evaluated the ability of the seven laboratories to reproduce relative potencies among themselves and against the consensus relative potencies using the estimated values from the ANOVA models.

\section{P055}

\section{ROTAVIRUS SEROTYPES: RESULTS FROM AN IMPACT} EMERGENCY DEPARTMENT STUDY

AK McDermid $^{1}$, NM Le Saux ${ }^{2}$, J Bettinger ${ }^{3}$, P Dery ${ }^{4}$, J Embree ${ }^{1}$, W Vaudry ${ }^{5}$, S Halperin ${ }^{6}$, $T$ Booth ${ }^{1}$

${ }^{1}$ Winnipeg, MB; ${ }^{2}$ Ottawa, ON; ${ }^{3}$ Vancouver, BC; ${ }^{4}$ Quebec, QC; ${ }^{5}$ Edmonton, $\mathrm{AB} ;{ }^{6} \mathrm{Halifax}, \mathrm{NS}$

BACKGROUND: Rotaviruses demonstrate substantial diversity which varies by geographic region and over time. Group A viruses commonly causing human disease are typed according to capsid proteins designated as $P$ and $G$ proteins. These are critical targets of antibody production in disease prevention as well as vaccine development. Knowledge of the circulating serotypes within a country will be useful in determining potential vaccine efficacy and in assessing future needs as other serotypes emerge.

METHODS: 5 pediatric hospitals from 5 provinces within the IMPACT network over 3 years (2007-9) collected isolates and clinical data on outpatients with diarrhea presenting to the Emergency Department for 
treatment. The National Microbiology Lab in Winnipeg, Manitoba sequenced rotavirus using VP7 segment and VP4 segment sequencing. RESULTS: 291 samples (189 screened positive for rotavirus and 102 that screened negative) were analysed. 186 specimens were genotype positive out of 189 that screened positive (98.4\%) by rapid testing. 40 genotyped positive out of $102(39.6 \%)$ that screened negative by rapid testing. 14 of $226(6.2 \%)$ positively genotyped samples were co-infected with greater than one serotype. 198 (87.6\%) rotaviruses from separate specimens were confirmed by sequencing. Of the non-coinfected samples, 139 (61.5\%) G1P[8] , 36 (15.9\%) G3P[8], 19 (8.4\%) G2P[4], 14 (6.2 \%) G9P[8] and 3 (1.3\%) G4P[8] genotypes were detected, as well as $1(0.4 \%)$ G1P[4] genotype. G9P[8] strains were found only in Ontario and Nova Scotia. The proportion of G3P[8] differed by province over the three year period.

CONCLUSIONS: This is the first nationally representative sample of rotavirus serotypes in Canada and provides baseline data of circulating rotavirus strains in Canada. One third of negative stool specimens contained rotavirus by molecular testing suggesting that a significant proportion of patients may not be captured with current clinical diagnostic testing. G1P[8] serotype is the most common followed by G3P[8] and G2P[4]. G9P[8] considered an emerging serotype, represented 6\% of the sample. This serotype is not currently represented in licensed vaccines. Continued surveillance should be implemented as vaccine use increases.

\section{P056}

UPDATE ON IMPACT SURVEILLANCE OF HAEMOPHILUS INFLUENZAE ISOLATES IN CANADA

M Shuel $^{1}$, B Tan ${ }^{2}$, D Scheifele ${ }^{3}$, D Law ${ }^{1}$, W Vaudry ${ }^{4}$, S Halperin $^{5}$, K Grimsrud $^{6}$, B Bortolussi ${ }^{5}$, R Pless ${ }^{6}$, R Tsang ${ }^{1}$

${ }^{1}$ Winnipeg, MB; ${ }^{2}$ Saskatoon, SK; ${ }^{3}$ Vancouver, BC; ${ }^{4}$ Edmonton, AB; ${ }^{5}$ Halifax, NS; ${ }^{6}$ Ottawa, ON

BACKGROUND: Conjugate vaccines against $H$. influenzae serotype b have been used in Canada since the early 1990s. The IMPACT program for prospectively monitoring all invasive $H$. influenzae infections regardless of serotype has been in place since 2007. Isolates from 12 pediatric centres across Canada are collected and sent to the National Microbiology Laboratory for further characterization.

PURPOSE: To monitor and report the phenotypic and genotypic nature of the isolates causing invasive $H$. influenzae disease in Canada's population.

METHODS: Isolates were confirmed to be H. influenzae by biochemical testing and $16 \mathrm{~S}$ rRNA sequencing and were characterized by serotype, biotype, multilocus sequence typing (MLST) and antibiotic susceptibility testing.

RESULTS: From January 2007 to March 2009, 68 isolates were collected from individual disease cases. Patient ages ranged from 1 day to 14 years. There were 22 serotype a, 10 serotype b, 1 serotype c, 12 serotype $\mathrm{f}$, and 23 non-typeable isolates. Seventeen isolates were recovered from cerebrospinal fluid, including 10 serotype a, 3 non-typeable and 2 each of serotypes $b$ and $f$. MLST data suggests that isolates belonging to the same serotype are highly related, while non-typeable isolates showed a higher degree of diversity. Antibiotic susceptibility testing showed that resistance was most common in serotype b and nontypeable isolates.

CONCLUSIONS: Non-typeable and serotype a strains each accounted for about one third of the invasive $H$. influenzae disease cases observed during the study period. Serotype $f$ strains caused another $18 \%$; and after two decades of $H$. influenzae serotype $b$ vaccination in Canada, serotype b accounted for $15 \%$ of the cases. Continued laboratory surveillance of H. influenzae at IMPACT centres is important to monitor further potential changes in the epidemiology of this invasive pathogen in the post-vaccination era.
P057

VACCINE PRESSURE AND IMMUNE SELECTION ON VACCINE PREVENTABLE BACTERIAL DISEASES AGENTS

\section{RS Tsang}

\section{Winnipeg, MB}

BACKGROUND: Immune selection on bacteria may result from natural immunity developed in the population or from vaccine pressure. PURPOSE: This study examines how immune pressure affects a few vaccine preventable bacterial disease agents.

METHODS: Examples from literature were used to illustrate bacteria adaptation to vaccine pressure and immune selection.

RESULTS: In Streptococcus pneumoniae causing invasive pneumococcal disease, introduction of the heptavalent pneumococcal conjugate vaccine (PCV7) has led to the emergence of diseases due to either vaccine-related pneumococcal serotypes or non-vaccine serotypes. Capsule switching has also been demonstrated in disease-causing pneumococci.

Changes in the epidemiology of invasive Haemophilus influenzae disease, including serotype replacement, have been reported from several countries, including Canada, in the post H. influenzae serotype b (Hib) vaccination era.

In Bordetella pertussis, many genetic polymorphisms have been described, involving such diverse genes like fimbriae, pertussis toxin, and pertactin that encode for the virulence factors (vaccine components). Analysis of genomic DNA from B. pertussis strains by pulsed-field gel electrophoresis is also able to differentiate between recent clinical isolates and strains used in the manufacturing of pertussis vaccines. These genetic changes have been suggested to be responsible for the resurgence of pertussis in countries with high vaccine coverage.

After more than a decade of endemic disease and many localized outbreaks, genetic and antigenic changes of sub-capsular protein antigens (involving the serotype and serosubtype antigens as well as the ironregulated outer membrane proteins such as FetA) were detected in the serogroup C ET-15 Neisseria meningitidis strains. These genetic changes causing antigenic shifts may explain increased disease activities in the early 2000 in Canada and the U.S.

CONCLUSION: Since bacterial adaptation to immune selection may lead to vaccine escape mutants, it is essential for public health laboratories to have a system to monitor the evolving bacteria for causing potential vaccine breakthrough cases.

\section{P058}

\section{A PLANT-MADE VIRUS-LIKE-PARTICLE VACCINE EXPRESSING eGFP: A NEW TOOL TO TRACK IMMUNE RESPONSES}

K Young ${ }^{1}$, M Couture ${ }^{2}$, M D'Aoust ${ }^{2}$, P Lavoie'2 ${ }^{2}$ L Vézina ${ }^{2}$, B Ward ${ }^{1}$

${ }^{1}$ Montreal, QC; ${ }^{2}$ Sainte Foy, QC

BACKGROUND: Pandemic influenza remains a constant threat and vaccine stockpiling is a high priority for international health organizations. Pandemic preparedness requires an effective vaccine that can be developed rapidly and in large quantities. Unfortunately, technologies currently used for influenza vaccine production are unable to meet these standards. As a result, there is a great need for new technologies. A new H5N1 influenza vaccine candidate has recently been developed by Medicago Inc. based upon virus-like-particles (VLP) produced in tobacco plants (Nicotania benthamiana). These VLP have demonstrated unexpectedly strong immunogenicity in pre-clinical models and a Phase I human trial. However, the mechanism of action underlying their potent immunogenicity is still unknown. We therefore wish to generate a plant-derived H5/eGFP-based VLP reagent, which will be a powerful tool for studying immune responses induced by VLP.

METHODS: H5 and eGFP coding regions were fused and cloned into a binary cowpea mosaic virus (CPMV)-based expression cassette. Agrobacteria (Agrobacterium tumefasciens) transformed with the 
resulting plasmid were inoculated into mature Nicotiana benthamiana plants by syringe infiltration. Six days post-inoculation, plants were harvested and protein content was extracted from leaf tissue for analysis.

RESULTS: The H5/eGFP fusion protein was stably expressed in tobacco plant cells. Formation of a H5/eGFP-based VLP was confirmed by confocal fluorescence microscopy, spectrofluorometry, size-exclusion chromatography, dynamic light-scattering, and electron microscopy. Fluorescent VLP were capable of hemagglutination, indicating the $\mathrm{H} 5$ molecule had maintained in vitro function.

CONCLUSIONS: We have successfully generated an eGFP-VLP reagent that is capable of interacting in vitro in the same way as normal influenza VLP. This reagent will be a useful tool to track the immune course of the VLP at both the cellular level and in an animal model. Understanding the immunological properties of this first, plant-made VLP will provide a better understanding of the capacity of this novel platform technology to produce viable vaccine candidates.

\section{PROGRAMMATIC EVALUATION AND} PUBLIC HEALTH POLICY

\section{P059}

REVIEW OF PROVINCIAL PUBLIC HEALTH POLICIES: MINIMIZING THE RISK OF POLIOMYELITIS TO

\section{ONTARIANS}

\section{E Bontovics}

\section{Toronto, ON}

BACKGROUND: There are ongoing outbreaks of laboratory confirmed wild poliovirus type 1 in Asia. Canada was declared polio free in 1995. The last case of polio in Ontario occurred in 1988 in a partially immunized infant as a result of wild poliovirus importation.

PURPOSE: To assess public health policies for minimizing the risk of poliomyelitis to Ontarians

METHODS: Review of provincial public health policies targeted at minimizing risk of poliomyelitis

RESULTS: In Ontario children are routinely immunized against polio beginning in infancy. All children attending a licensed child care are required to be up-to-date on immunizations against polio under the Day Nursery Act. Under the Immunization of School Pupils Act, children are required to be immunized against poliovirus, or have a valid exemption, in order to attend school. Immunization data are collected yearly from each health unit to assess provincial polio vaccine coverage. Vaccine coverage is high in the majority of health unit jurisdictions $(56 \%$ of health units have greater than $95 \%$ coverage, and $86 \%$ of health units have greater than $90 \%$ coverage for 7 to 17 year olds in the 2008/2009 school year, 94\% coverage for 17 year olds for 2008-09).

There are small pockets of under immunized communities in Ontario who do not immunize their children for religious or philosophical reasons who may be at risk.

Ontario takes part in the national surveillance program monitoring all cases of flaccid paralysis in children under 15 years old.

The ministry has established a policy for local health units for the prompt follow up of both the Sabin vaccine-like strain and vaccine derived poliovirus (VDPV) imported to Ontario.

CONCLUSIONS: There are public health policies in place to maintain high vaccine coverage, prompt detection and appropriate management for polio importation. Polio outbreaks in Asia pose a risk for under-immunized Ontarians.
P060

IMPACT DU RETARD À L'ADMINISTRATION DES VACCINS PRÉVUS À LA VISITE DE 2 MOIS SUR LA COUVERTURE VACCINALE DES JEUNES ENFANTS QUÉBÉCOIS N Boulianne $^{1}$, D Audet ${ }^{1}$, R Bradet ${ }^{1}$, G Deceuninck ${ }^{1}$, M Guay ${ }^{2}$, M Ouakki ${ }^{1}, \mathrm{G}$ De Serres ${ }^{1}$

${ }^{1}$ Québec, QC; ${ }^{2}$ Sherbrooke, QC

CONTEXTE ET OBJECTIFS : Au Québec, différentes mesures sont déployées en vue de diminuer les retards vaccinaux chez les nourrissons. Nous avons profité des enquêtes bisannuelles de couverture vaccinale (CV) de 2008 et de 2010 afin d'évaluer l'impact sur la CV d'un retard d'un mois à l'administration des premiers vaccins prévus à l'âge de 2 mois.

MÉTHODE : Deux échantillons de 1000 enfants âgés d'un an et de deux ans provenant du fichier de la régie de l'assurance maladie du Québec pour les deux années à l'étude ont été constituées.

Un questionnaire postal auto-administré reproduisant le carnet de vaccination a été complété par les parents. Avec l'autorisation de ces derniers, les vaccins manquants étaient recherchés auprès des vaccinateurs. La (CV) complète à 15 mois (cohorte un an) et à 24 mois (cohorte deux ans) a été évaluée ainsi que différents facteurs associés à la CV. Des analyses univariée et multivariée ont été produites.

RÉSULTATS : Le taux de réponse est de $65 \%$ en 2008 et de $63 \%$ en 2010*. La (CV) complète à l'âge de 15 mois est passée de $75 \%$ en 2008 à $82 \%$ en $2010 \%$. Celle à 24 mois, de $78 \%$ à $79 \%$. En 2008, parmi les enfants ayant reçu leur premier vaccin avant l'âge de 3 mois, la CV à 24 mois est de $81 \%$ vs $36 \%(\mathrm{p}<0,0001)$ chez ceux vaccinés à 3 mois et plus (analyse univariée). En 2010, ces proportions sont respectivement de $82 \%$ et $44 \%(p<0,0001)$. Selon la cohorte, en $2008,18 \%$ et $11 \%$ des parents rapportaient un retard au premier vaccin comparativement à $10 \%$ et $13 \%$ en 2010 . La raison évoquée par près de $50 \%$ des parents étant la difficulté à obtenir un rendez-vous.

CONCLUSION : Pour diminuer les délais dans l'administration des vaccins et améliorer la couverture vaccinale, une plus grande accessibilité aux services de vaccination et l'utilisation d'un système de rappel et de relance pourraient être indiquées.

* Données préliminaires pour 2010.

\section{P061}

COST-EFFECTIVENESS OF ONE- AND TWO-DOSE VARICELLA VACCINATION: INCLUDING DYNAMIC POPULATION EFFECTS ON VARICELLA AND HERPES ZOSTER

\author{
M Brisson, G Melkonyan, M Drolet, G De Serres, P De Wals \\ Quebec, QC
}

BACKGROUND: In many countries, policymakers are being asked to make recommendations regarding the introduction of a 2-dose varicella vaccination program.

PURPOSE: The aim of this study was to examine the cost-effectiveness of 2-dose versus 1-dose varicella vaccination programs in Canada.

METHODS: A deterministic realistic age-structured model was developed to predict the impact of varicella vaccination on varicella and zoster. The model was calibrated to 1 -dose and 2-dose vaccine efficacy, force of infection and zoster incidence. Quality-Adjusted LifeYears (QALYs) and costs were estimated using data from the literature. The economic evaluation was conducted from the ministry of health perspective, the time horizon of the analysis was 80 years, and $5 \%$ discounting was used for costs and benefits.

RESULTS: At CAN $\$ 30$ per course, 1-dose infant vaccination (coverage $=90 \%$ ) is estimated to yield cost-effectiveness ratios below CAN $\$ 40,000$ per QALY-gained under a wide range of model assumptions. However, the incremental cost-effectiveness ratios of adding a second dose vary considerably (range from cost saving to producing overall losses in QALYs) and are highly sensitive to mixing patterns 
and assumptions regarding the impact of varicella vaccination on herpes zoster, and to a lesser extent to the discount rate, vaccine price, vaccine efficacy, and the severity of breakthrough varicella.

INTERPRETATION: Although a 2-dose program may help guarantee high population-level effectiveness, the incremental gains in effectiveness provided by the second dose are highly uncertain and may not be justified by its costs.

\section{P062}

EFFECTIVENESS OF SEROGROUP C MENINGOCOCCAL CONJUGATE VACCINE: A 7-YEAR FOLLOW-UP IN QUEBEC, CANADA

\section{P De Wals ${ }^{1,2}$, G Deceuninck ${ }^{1}$, B Lefèbvre ${ }^{2}$, N Boulianne ${ }^{1}$,} G De Serres ${ }^{1}$

${ }^{1}$ Quebec City, QC; ${ }^{2}$ Montreal, QC

BACKGROUND: In 2001, an outbreak of serogroup C meningococcal disease (C-MD) occurred in the province of Quebec and a mass immunization campaign was rapidly implemented, using serogroup $\mathrm{C}$ polysaccharide-CRM169 protein conjugate vaccine (C-MCV). In the fall of 2002, C-MCV was introduced in the routine schedule and one dose at age 12 months was recommended.

OBJECTIVES: To assess the impact of the mass campaign and C-MCV effectiveness over a 7-year period.

METHODS: Vaccination rates were estimated from the meningococcal vaccination registry established during the mass campaign and surveys conducted every two years in a random sample of children. C-MD cases were those notified to public health authorities and/or those ascertained by the provincial reference laboratory. Vaccine effectiveness (VE) was estimated using a cohort method, adjusting rates by the Mantel-Haenzel method or logistic regression.

RESULTS: From 1990 to 2008, 530 C-MD cases were identified, including 14 cases following C-MCV administration. The 2001 outbreak was rapidly controlled following the mass campaign and C-MD incidence declined in all age groups to reach a minimum of 6 cases in 2008. Over a 7 -year period, VE was $88.0 \%$ (95\% CI: $70.2 \%$ to $95.2 \%$ ). There was a trend of increasing protection according to age at vaccine administration and of waning of protection of a higher magnitude for children vaccinated at younger age as compared with older individuals. CONCLUSIONS: The impact of a mass immunization campaign followed by a routine one-dose program is similar to the impact of a mass campaign followed by a 3-dose routine program as observed in the UK. Adolescent revaccination is probably needed to maintain long-term protection.

\section{P063 \\ ASSESSING THE POTENTIAL IMPACT OF PUBLICLY FUNDED HPV VACCINATION AND CERVICAL SCREENING ON CERVICAL CANCER-RELATED HEALTH EQUITY IN ONTARIO}

\section{Doyle, $\mathrm{S}$ Deeks, $\mathrm{H}$ Manson \\ Toronto, ON}

BACKGROUND: Cervical cancer affects women inequitably; socially marginalized women develop cervical cancer more often, yet undergo screening less frequently. Whether school-based HPV vaccination programs exacerbate or diminish inequities is unknown.

OBJECTIVE: To examine the potential impact of school-based HPV vaccination and cervical screening on cervical cancer-related health equity in Ontario.

METHODS: Using published and grey literature, participation rates in school-based immunization and cervical cancer screening, as well as acquisition of cervical cancer, were analyzed, according to various social determinants of health indicators.

RESULTS: Cervical cancer incidence is highest among low-income, Aboriginal, geographic-remote, non-Caucasian, older, less educated and under-housed women. Cervical screening has decreased cervical cancer rates; however, lower participation of subpopulations, including women of older age, low-income, Aboriginal, under-housed, geographicremote, non-English, non-Caucasian, and those with a male physician, has been identified. School-based immunization programs can enhance equity by providing access to primary prevention for low-income, Aboriginal, non-Caucasian and geographic-remote individuals. Ogilvie and co-authors reveal that HPV vaccine coverage is lower among higher educated British Columbians (1), a group that tends to participate in screening. Published literature reveals different populations may access immunization and screening programs.

CONCLUSION: School-based immunization programs have the potential to enhance coverage and access to cervical cancer prevention among the socially marginalized. As different populations participate in primary and secondary prevention programs, there may be, higher than anticipated, decreases in cervical cancer incidence.

REFERENCE

1. Ogilvie, Gina et al. 2010. "A Population-Based Evaluation of a Publicly Funded, School-Based HPV Vaccine Program in British Columbia, Canada: Parental Factors Associated with HPV Vaccine Receipt" in PLoS Medicine, 7(5), 1-11.

\section{P064}

SURVEILLANCE OF ADVERSE EVENTS FOLLOWING IMMUNIZATIONS IN REGINA QU'APPELLE HEALTH REGION, REGINA, SASKATCHEWAN

\section{T Diener, Z Abbas, M Granger, D Martin}

\section{Regina, SK}

A retrospective review of adverse events following immunization (AEFI) for the period 1998-2008 was done using the adverse events following immunization (AEFI) data maintained by Population and Public Health Services, Regina Qu'Appelle Health Region (RQHR). METHODS: A descriptive record based review was used where the date of vaccination and onset of the adverse event occurred between January 1998 and December 2008. An AEFI was defined as any adverse event that occurred after a vaccination, which might be related to the vaccine itself or to its administration. Denominator for influenza vaccine was not available and was excluded from analysis.

RESULTS: A total of 817 AEFIs were identified corresponding to 506 individual clients who presented with one or more AEFIs for vaccines administered between January 1, 1998 and December 31, 2008. These AEFIs were from a total of 443,769 vaccine doses administered during this period. The overall reporting rate was 114 per 100,000 doses administered. The highest proportion of adverse events was among children aged $0-2$ years $(48.8 \%)$. The majority of the AEFIs described non-serious events and only four cases were hospitalized. One reported death was temporally associated with immunization but there were no evidence to suggest a causal association. The most common adverse event was rashes $(29.4 \%)$ followed by allergic reactions $(28.9 \%)$ and fever $(28.6 \%)$. Severe pain and swelling was reported in $23.7 \%$ of AEFI. Pentavalent vaccine was responsible for the highest number of AEFI reports (228) as a percentage of all AEFI reports (22.9\%). Mumps, measles, rubella (MMR) was the second most common vaccine associated with AEFI reports (132) and accounted for $24.7 \%$ of all reported AEFI.

CONCLUSION: Vaccines administered in RQHR have proven to be generally safe. The limitations of the current surveillance system and quality of data need improvements in the future. 
P065

PRIORITIES FOR NEW VACCINATION PROGRAMS IMPLEMENTATION: PAEDIATRICIANS' AND FAMILY PHYSICIANS' OPINIONS

E Dubé $^{1}$, V Gilca ${ }^{1}$, C Sauvageau ${ }^{1}$, N Boulianne ${ }^{1}$, FD Boucher ${ }^{1}$, JA Bettinger ${ }^{2}$, S McNeil ${ }^{3}$, I Gemmill ${ }^{4}$, F Lavoie ${ }^{1}$, M Ouakki ${ }^{1}$ ${ }^{1}$ Quebec, QC; ${ }^{2}$ Vancouver, BC; ${ }^{3}$ Halifax, NS; ${ }^{4}$ Kingston, ON

BACKGROUND AND AIMS: In Canada, several new vaccines were or will be soon approved and many new candidate public vaccination programs are in ongoing discussion. Health professionals' opinion toward new vaccines is well recognized to influence future decisions concerning immunization programs. We assessed paediatricians' and family physicians' perceived priority ratings for 7 new immunization programs implementation.

METHODS: A self-administered anonymous mail-based questionnaire was sent to 3034 Canadian physicians. Basic Priority Rating System (BPRS) approach was used. Responses to 8 statements regarding frequency and severity of the diseases, efficacy and safety of the vaccines and feasibility of immunization programs were used to calculate the BPRS scores (minimal score 0; maximal score 100).

RESULTS: 1283 physicians participated (43\%). Perceived usefulness of different new public immunization programs varied from $29 \%$ to $67 \%$. The BPRS scores were: 77 for the quadrivalent measles-mumps, rubella and varicella vaccine (MMRV); 75 for the hexavalent vaccine (DTaP-Polio-Hib-Hepatitis B); 72 for the conjugate pneumococcal vaccine (PCV-10); 69 for the quadrivalent meningococcal vaccine (ACYW135); 68 for the combined hepatitis A and B vaccine; 63 for the $\mathrm{HPV}$ vaccine and 56 for the rotavirus vaccine. BPRS scores were not influenced by respondents' sociodemographic or professional characteristics or by self-estimated level of knowledge on the vaccines. Between $0.24 \%$ and $2 \%$ of respondents considered that a given immunization program would not be feasible.

CONCLUSION: Physicians' opinions regarding usefulness and feasibility of new immunization programs varied greatly depending on the vaccine. As shown in previous studies, combined vaccines received the highest ratings.

\section{P066 \\ ROTAVIRUS VACCINATION: RESULTS OF A SURVEY ON CANADIAN PHYSICIANS' KNOWLEDGE, ATTITUDES AND PRACTICES \\ E Dubé $^{1}$, R Bradet ${ }^{1}$, V Gilca ${ }^{1}$, C Sauvageau ${ }^{1}$, N Boulianne ${ }^{1}$, FD Boucher ${ }^{1}$, JA Bettinger ${ }^{2}$, S McNeil ${ }^{3}$, I Gemmill ${ }^{4}$, F Lavoie $^{1}$ ${ }^{1}$ Quebec, QC; ${ }^{2}$ Vancouver, BC; ${ }^{3}$ Halifax, NS; ${ }^{4}$ Kingston, ON}

BACKGROUND AND AIMS: Almost all young children are affected by rotavirus and more than half of cases seek medical care. In the context where rotavirus immunization is recommended, but not publicly funded, vaccine uptake depends largely upon whether health professionals recommend it to parents. We assessed paediatricians' and family physicians' knowledge, attitudes and intentions regarding rotavirus vaccines.

METHODS: A self-administered anonymous mail-based questionnaire based upon the Health Belief Model was sent to a sample of 1182 family physicians and to all paediatricians.

RESULTS: Between $79 \%$ and $90 \%$ of respondents agreed with the statements regarding the health and economic burden of rotaviral disease and $77 \%$ had had experience with severe cases in their medical practice. Consequences of rotavirus infection among patients under the age of 3 were rated as mild (48\%) or moderate (41\%). Respectively $96 \%$ and $93 \%$ of the physicians perceived the rotavirus vaccines as safe and effective, and $80 \%$ estimated that their knowledge on rotavirus vaccines was sufficient. The majority of respondents (80\%) intended to recommend rotavirus vaccines to their patients. Intention to recommend rotavirus vaccines was independently associated $(\mathrm{p}<0.0001)$ with: perceived benefits of vaccination (partial R2 $=0.55$ ); perceived acceptability of the vaccine by vaccinators (partial R2=0.05); self-estimated sufficiently of knowledge about rotavirus vaccines (partial R2=0.01); perceived severity of rotaviral diseases (partial R2=0.01).

CONCLUSION: Most paediatricians and family physicians manifested willingness to recommend the rotavirus vaccine. Although consequences of rotavirus gastroenteritis were perceived as mild or moderate, immunization against rotavirus was seen as beneficial for young children.

\section{P067}

CANADIAN FAMILY PHYSICIANS' AND PAEDIATRICIANS' OPINIONS AND INTENTIONS REGARDING HEPATITIS A AND B INFECTION AND THEIR PREVENTION BY VACCINATION E Dubé $^{1}$, V Gilca ${ }^{1}$, C Sauvageau ${ }^{1}$, N Boulianne ${ }^{1}$, FD Boucher ${ }^{2}$, JA Bettinger ${ }^{2}$, S McNeil ${ }^{3}$, I Gemmill ${ }^{4}$, F Defay ${ }^{1}$, F Lavoie ${ }^{1}$ ${ }^{1}$ Quebec, QC; ${ }^{2}$ Vancouver, BC; ${ }^{3}$ Halifax, NS; ${ }^{4}$ Kingston, ON

BACKGROUND: The strategies of hepatitis $\mathrm{A}$ and $\mathrm{B}$ prevention by vaccination are heterogeneous among Canadian provinces. Hepatitis $B$ public vaccination programs for infants exist in 3 Canadian provinces and 3 territories, and school-based universal programs in 7 other provinces. All provinces but Quebec have exclusively selective vaccination against hepatitis A. In Quebec, a two-dose vaccination schedule with a combined hepatitis A and B vaccine was implemented in 2008.

PURPOSE: To assess clinicians' opinions regarding hepatitis $\mathrm{A}$ and $\mathrm{B}$ infection and their prevention through a publicly funded immunization program with a combined hepatitis $\mathrm{A}$ and $\mathrm{B}$ vaccine.

METHODS: An anonymous questionnaire based upon the Erickson and De Wals framework was mailed in fall 2009 to a random sample of family physicians and all Canadian paediatricians. Descriptive statistics were generated for all variables. Multiple regression analysis was used to determine variables independently associated with the perceived usefulness of vaccine implementation.

RESULTS: Participation rate was 42.6\% (1260/2959). Depending on the province/region of practice, between 44 and $73 \%$ of respondents were directly involved in vaccination. 85 to $90 \%$ of respondents perceived hepatitis A and B as serious diseases and 55 to $65 \%$ as diseases that generate a significant economic burden. The combined vaccine was perceived as safe by $91-94 \%$, effective by $91-96 \%$; and well accepted by vaccinators $90-95 \%$; 86 to $90 \%$ of respondents manifested willingness to recommend the vaccine and $80-84 \%$ considered that it will be very useful to implement a publicly funded vaccination program with a combined hepatitis $\mathrm{A}$ and $\mathrm{B}$ vaccine.

DISCUSSION: A high homogeneity of results was observed among Canadian provinces in this survey. The great majority of surveyed clinicians, mostly involved in vaccination, perceive the implementation of a public immunization program with a combined hepatitis $\mathrm{A}$ and $\mathrm{B}$ vaccine as a feasible and useful public health intervention.

\section{P068 \\ FIABILITÉ D'UN QUESTIONNAIRE SUR LES DÉTERMINANTS DE LA VACCINATION VPH D'ADOLESCENTES QUÉBÉCOISES}

$\underline{M}$ Guay $^{1,2}$, J Lemaire ${ }^{1}$, P Clément ${ }^{2}$, A Hamid ${ }^{2}$, Y Tahmi $^{1}$

${ }^{1}$ Longueuil, QC; ${ }^{2}$ Québec, QC

CONTEXTE : Malgré plusieurs études sur la vaccination des adolescentes contre le virus du papillome humain (VPH), aucune n'aurait employé de questionnaire validé. Dans le cadre d'une vaste enquête québécoise, une étude a été réalisée sur les qualités métrologiques du questionnaire à utiliser.

OBJECTIF : Évaluer la fiabilité d'un questionnaire en français sur les déterminants de la vaccination VPH.

MÉTHODE : Il s'agit d'une étude test-retest réalisée à l'automne 2009 auprès de 110 élèves de 3 e secondaire. Le questionnaire étudié a été élaboré à partir d'autres études et du Modèle des croyances relatives à la santé (Health Belief Model). Le test a été réalisé sur la version papier 
du questionnaire lors de la vaccination VPH offerte à l'école. Le retest a été rempli en ligne sur Internet après deux semaines. Des tests de kappa ont comparé les résultats test-retest.

RÉSULTATS : Le taux de réponse a été de $61 \%$ au « test » $(67 / 110)$ puis de $82 \%(55 / 67)$ au « retest ». Les questions sur les connaissances et l'histoire vaccinale sont moins fidèles; celles sur les raisons de nonvaccination et données sociodémographiques sont plus fidèles.

\begin{tabular}{|c|c|c|c|c|c|}
\hline $\begin{array}{l}\text { Catégories de } \\
\text { variables }\end{array}$ & $\begin{array}{l}\text { Nombre de } \\
\text { questions } \\
\text { concernées }\end{array}$ & $\begin{array}{l}\text { Meilleur } \\
\text { kappa }\end{array}$ & $\begin{array}{l}\text { Intervalle } \\
\text { de } \\
\text { confiance }\end{array}$ & $\begin{array}{l}\text { Moins } \\
\text { bon } \\
\text { kappa }\end{array}$ & $\begin{array}{l}\text { Intervalle de } \\
\text { confiance< }\end{array}$ \\
\hline Connaissances & 10 & 0,59 & $0,35-0,82$ & 0,23 & $-0,03-0,49<$ \\
\hline $\begin{array}{l}\text { Perception de la } \\
\text { vulnérabilité et de la } \\
\text { sévérité de la maladie }\end{array}$ & 4 & 0,54 & $0,32-0,77$ & $-0,06$ & $-0,16-0,04<$ \\
\hline $\begin{array}{l}\text { Perception des } \\
\text { bénéfices et des } \\
\text { coûts du vaccin }\end{array}$ & 5 & 0,56 & $0,33-0,79$ & 0,25 & $-0,05-0,45<$ \\
\hline Influence des parents & 4 & 1,00 & $1,00-1,00$ & 0,51 & $0,21-0,80<$ \\
\hline $\begin{array}{l}\text { Facteurs ayant } \\
\text { influencé la décision } \\
\text { de vaccination }\end{array}$ & 7 & 0,52 & $0,34-0,70$ & $-0,06$ & $-0,21-0,09<$ \\
\hline $\begin{array}{l}\text { Facteurs ayant } \\
\text { influencé la décision } \\
\text { de non-vaccination }\end{array}$ & 6 & 1,00 & $1,00-1,00$ & 0,64 & $0,05-1,00<$ \\
\hline $\begin{array}{l}\text { Histoire antérieure de } \\
\text { vaccination }\end{array}$ & 3 & 0,16 & $-0,12-0,44$ & $4 \quad-0,04$ & $-0,09--0,01<$ \\
\hline $\begin{array}{l}\text { Données } \\
\text { sociodémographiques }\end{array}$ & 8 & 1,00 & $1,00-1,00$ & 0,66 & $-0,03-1,00$ \\
\hline
\end{tabular}

CONCLUSION : Il est pertinent d'évaluer un questionnaire avant de l'employer à large échelle; il a été ajusté notamment pour les questions sur l'histoire vaccinale. Une telle évaluation devrait être faite lors d'études sur les déterminants de la vaccination.

\section{P069}

DETERMINANTS OF VACCINATION AGAINST THE HUMAN PAPILLOMAVIRUS AMONG QUEBEC TEENAGERS: PARENTAL AND TEEN PERSPECTIVES

M Guay ${ }^{1}$, P Clément ${ }^{2}$, A Hamid² ${ }^{2}$ È Dubé2 ${ }^{2}$, C Sauveageau ${ }^{2}$,

N Boulianne ${ }^{2}, M_{\text {Landry }}{ }^{3}$, J Lemaire ${ }^{1}$

${ }^{1}$ Longueuil, QC; ${ }^{2}$ Quebec, QC; ${ }^{3}$ Montreal, QC

CONTEXT: The implementation of vaccination against the human papillomavirus (HPV) in Canada was criticized. Despite this, in 20082009, 81\% of Quebec female students in their third year of high school (equivalent to Grade 9) had received complete HPV vaccination.

OBJECTIVE: To explore the determinants of HPV vaccination among Quebec teenagers.

METHOD: To prepare a major Quebec study, focus groups were conducted in the fall of 2009 with teenagers (aged 15 to 19, vaccinated and non-vaccinated) and parents of teenagers (vaccinated and nonvaccinated). The discussion guide was developed following an extensive literature review and from a model adapted from the Health Belief Model and the Walsh and McPhee model, which takes into account the mutual influence of teenagers and their parents, as well as interactions with health care professionals, on the vaccination decision. Recorded transcripts of the focus groups were summarized, re-transcribed and then submitted to content analysis.

RESULTS: Three focus groups were conducted (17 participants). All stated that they lacked information on HPV, the vaccine and free access to it. The vaccine's benefits were recognized, but concerns about its innocuousness existed. Doctor or parental recommendation is essential but the final decision lies with the teenager. School vaccination is appreciated and facilitates access, but vaccinated teens deplored the lack of privacy involved and the impression of being pressured into vaccination. Since sound sexual education is no guarantee against sexually transmitted infections, the parents of vaccinated teenagers consider that the vaccine protects their daughter's health.

CONCLUSION: Despite favourable acceptance of the HPV vaccine, information and accessibility must be enhanced (e.g. teenagers eligible for free vaccination outside of school) to allow teenagers and their parents to make an informed decision.

\section{P070}

\section{THE ROLE OF ECONOMICS IN IMMUNIZATION POLICY: INTERNATIONAL COMPARISONS}

\section{P Jacobs, A Ohinmaa \\ Edmonton, AB}

BACKGROUND: Since the beginning of the new millennium, prices of vaccines have been increasing significantly, reflecting decades of research and development. As they have done with pharmaceuticals, governments have begun to incorporate economic considerations into scientific reviews. Vaccines fall into the public health category, whose ethic differs historically from that in the pharmaceutical market.

PURPOSE: The purpose of this paper is to review how countries have incorporated economics into the scientific vaccine review process.

METHODS: We contacted experts in countries which had scientific review committees according to the VENICE website, and the USA, Australia, New Zealand, and Hong Kong. We asked a series of questions regarding the structure of the review process, the role of economics, and the economic criteria used.

RESULTS: We obtained information from ten countries which used economics in their vaccine scientific reviews. In terms of structure, reviews fell into two groups - those which incorporated economics into the scientific review (GB, FI, FR, HK, NL, NZ, US) and those which separated economic and clinical considerations (SW, AU). The criteria used in economic studies followed the pharmaceutical guidelines in all instances.

CONCLUSIONS: Pharmaco-economics has grown up in the world of pharmaceuticals, not in the public health arena. The ethics in these two arenas differ. Certain elements - epidemiological considerations, herd immunity, long effect times - are relevant to vaccines. Using pharmacoeconomic rules for vaccines may not create a level playing field.

\section{P071}

\section{WILL PANORAMA BE GOOD FOR US? - EVALUATION INDICATORS FOR ASSESSING THE IMPLEMENTATION OF A NEW IMMUNIZATION REGISTRY}

\section{J Kaashoek, J Cameron, V Dubey}

\section{Toronto, ON}

BACKGROUND: Toronto Public Health (TPH), along with Public Health Units in Ontario, will implement a new immunization information system (IIS) called Panorama in 2011. A good immunization information system (IIS) improves the quality of immunization services, data and population immunization outcomes. Implementing a new system is complex and will have many implications.

PURPOSE: To describe evaluation indicators for the implementation of Panorama in Vaccine Preventable Diseases (VPD) Program at TPH.

METHODS: Meetings with key stakeholders were conducted to identify the main areas of evaluation. A logic model was developed with key process and outcome indicators. The logic model was presented to stakeholders for additional feedback and revision.

RESULTS: A program logic model for Panorama implementation identified key outcomes. Short term outcomes focused on the process for implementation including impact on continuing and postponing current programs through implementation and staff knowledge. Shortterm indicators included the percentage of staff satisfied with using the panorama database; the percentage of staff who report being able to provide a timely response and good customer service to clients. 
Medium and long term outcomes looked at the impact of a new IIS on program delivery and immunization coverage outcomes. Medium and long-term indicators included the identification of low immunization coverage among communities and populations in Toronto, percentage reduction in the number of children being suspended from school for incomplete immunization records; and percentage improvement in vaccine delivery.

CONCLUSION: Implementing a new IIS for a large vaccine preventable disease program requires much advanced planning. During implementation, resources must be diverted and current programs put on hold. However, long-term outcomes should be able to assess if a new IIS was able to improve service delivery and ultimately improve immunization coverage in the population.

\section{P072 \\ HOSPITALIZATIONS ASSOCIATED WITH A DIAGNOSIS OF PNEUMONIA IN RELATION TO PNEUMOCOCCAL CONJUGATE VACCINE (PCV) USE IN THE PROVINCE OF ONTARIO, CANADA}

N Khuc ${ }^{1}$, N Rawson ${ }^{2,3}$, A McGeer ${ }^{4}$, P De Wals ${ }^{5}$

${ }^{1}$ Rennes, France; ${ }^{2}$ Mississauga, ON; ${ }^{3}$ Waterloo, ON;

${ }^{4}$ Toronto, ON; ${ }^{5}$ Quebec City, QC

BACKGROUND: PCV7 was licensed in Canada in May 2001 and was included in the infant immunization program in Ontario in February 2005 with no catch-up for older children.

PURPOSE: To assess the impact of PCV on hospital admission for pneumonia taking into account other factors.

METHODS: Hospital admissions with a main diagnostic code of pneumonia were obtained from the Canadian Institute for Health Information and population denominators from Statistics Canada. The monthly rate of admissions from April 1999 to March 2009 was analyzed using structural time-series models taking into account secular trends, seasonal effect, breaks in baseline and outliers. In addition, the shift from a high risk to a universal strategy for influenza immunization in 2000 was controlled using a categorical (before-after) or continuous variable (estimated uptake rates) affecting winter months only. The candidate explanatory variable was the PCV uptake rate defined as a categorical (accessibility of vaccine) or a continuous variable (estimated uptake rates).

RESULTS: Mean admissions rates were respectively 45.8/100,000, $5.5 / 100,000$ and $69.6 / 100,000$ person-years in the groups $<5,20-59$ and $60+$ years. There was a strong seasonal effect and influenza winter peaks were observed. There was a linear downward trend in all age groups, but no statistically significant effect of influenza or PCV7 immunization could be identified.

CONCLUSIONS: Seasonal effect is the major factor in the most of dynamic modelisation. No major impact of influenza or PCV7 immunization could be detected on hospital admissions for all-cause pneumonia in Ontario using a time-series modeling. The selected outcome is probably not very specific for influenza and pneumococcal infections but is of considerable interest for public health and health service evaluation.

\section{P073}

RESULTS FROM THE 2008 ADULT NATIONAL IMMUNIZATION COVERAGE SURVEY

\section{J Laroche, A Frescura, L Belzak \\ Ottawa, ON}

BACKGROUND: The Adult National Immunization Coverage Survey (aNICS) is implemented every two years by the Public Health Agency of Canada to assess immunization coverage in the general adult population and in specific targets groups such as health care workers (HCW), adults with chronic medical conditions, and seniors (aged $\geq 65$ years) and to monitor progress and attitudes towards national targets for immunization coverage.
PURPOSE: To present results from the 2008 aNICS. Results from the 2008 aNICS were statistically compared to results from previous survey cycles of the aNICS to assess performance and trends in select adult vaccine coverage over time.

METHODS: The aNICS uses random-digit-dialing and telephone interviews to reach a representative sample of 3000 Canadian adults to assess immunization coverage. HCW were selected from sampling frames from professional databases. As personal immunization records are not routinely kept by Canadian adults, respondents were asked to report on their immunization history based on recall. Linear regression was used to examine factors impacting vaccine uptake.

RESULTS: In the general population, vaccine coverage estimates for influenza (37\%), hepatitis B (36\%), tetanus (50\%), varicella (19\%), and pertussis (4\%) vaccines obtained from the 2008 aNICS were similar to coverage estimates obtained from the 2006 aNICS. Amongst HCW, hepatitis B coverage modestly increased between 2006 (62\%) and $2008(69 \%)$. Although an increase in influenza vaccine coverage was observed in HCW with close contact with patients between 2001 (55\%) and $2006(70 \%)$, influenza vaccine coverage remained stable between 2006 (70\%) and 2008 (72\%).

CONCLUSION: In the general population, vaccine coverage estimates from the 2008 aNICS are comparable to vaccine coverage estimates obtained in previous cycles of the aNICS. A modest increase in hepatitis B vaccine coverage was observed in $\mathrm{HCW}$. Nevertheless, vaccine coverage remains below national targets for all vaccines for which national targets have been established.

\section{P074 \\ CANADA'S NATIONAL ADVISORY COMMITTEE ON IMMUNIZATION (NACI): EVIDENCE-BASED DECISION MAKING ON VACCINES AND IMMUNIZATION SJ Ismail ${ }^{1,2,3}$, JM Langley ${ }^{4}, \underline{\text { T Harris }^{1}}{ }^{1}$, B Warshawsky ${ }^{5}$, S Desai ${ }^{1}$, M FarhangMehr ${ }^{1}$ ${ }^{1}$ Ottawa, ON; ${ }^{2}$ Edmonton, AB; ${ }^{3}$ Calgary, AB; ${ }^{4} \mathrm{Halifax}, \mathrm{NS}$; ${ }^{5}$ London, ON}

BACKGROUND: The structures, processes, and experiences of fifteen well-established National Immunization Technical Advisory Groups (NITAGs) from around the world were compiled in a recent supplement of the journal Vaccine as part of the Supporting national Independent Immunization Advisory Committees (SIVAC) Initiative, which aims to enhance the use of evidence-based decision making in the development of immunization programs and policies. An article on Canada's National Advisory Committee on Immunization (NACI) was included in the Vaccine supplement.

PURPOSE: The purpose of this article is to describe the structure and processes of NACI, as well as its approach to evidence-based decision making.

METHODS: A review of NACI's terms of reference, policies, and other documentation was conducted to highlight information on the description and background of the committee, processes, recommendation development, and challenges.

RESULTS: NACI is a respected, credible, scientific advisory committee of dedicated expert members. The committee was formed in 1964 and has reported to the Chief Public Health Officer of Canada since 2004. In 2009, NACI formally introduced its process to develop and grade evidence-based recommendations. Members consider various scientific factors (e.g. disease burden, vaccine characteristics) when making recommendations that are published in an open-access electronic periodical and summarized in the Canadian Immunization Guide. NACI recommendations are seen in many cases as setting a standard of care and are used by provinces, territories, professional associations, advocacy groups and individual care providers.

CONCLUSIONS: Although improved and more transparent evidence-based NACI decision making processes are now in place, challenges remain. While the committee has faced challenges in efficiently fulfilling its mandate in an increasingly complex immunization 
environment, it has been successful in providing relatively timely evidence-based immunization recommendations to Canadians in collaboration with other stakeholders and groups. Making populationlevel recommendations without formally considering the full spectrum of public health science presents challenges in the implementation of NACI's recommendations.

\section{P075}

RESULTS FROM THE 2006 AND 2009 CHILDHOOD NATIONAL IMMUNIZATION COVERAGE SURVEYS

\section{Laroche, A Frescura, L Belzak \\ Ottawa, ON}

BACKGROUND: Childhood National Immunization Coverage Surveys (cNICS) are implemented every two years by the Public Health Agency of Canada to assess up-to-date and on-time vaccine coverage of children 2, 7, and 17 years of age as well as changes in parental knowledge, attitudes and beliefs (KAB) about immunization, and to monitor progress towards national targets for immunization coverage.

PURPOSE: To present the results from the 2009 cNICS, as well as revised results from the $2006 \mathrm{cNICS}$. Results will be compared with provincial and territorial estimates and internationally to assess performance and trends in childhood vaccine coverage over time.

METHODS: The cNICS uses telephone interviews with parents/ guardians to obtain the immunization history of children. Respondents were asked to report from an immunization record or recall on the immunizations received by their child. Coverage was assessed from respondent reporting from an immunization record. Additional analysis will look at the impact of changing vaccine program policies and sampling methodology on vaccine coverage estimates.

RESULTS: Coverage estimates from the cNICS increased significantly for the newer vaccines (meningococcal conjugate, varicella, and pneumococcal conjugate). Results from the $2006 \mathrm{cNICS}$ revealed that vaccine coverage for the more established vaccines, such as diphtheriatetanus-acellular pertussis and measles-mumps-rubella had not changed. Vaccine coverage for routine childhood immunizations in 7 and 17 year old children did not change significantly between 2001 and 2006. Results for the 2009 cNCIS are being analyzed and validated.

CONCLUSION: National vaccine coverage results for routinely administered childhood vaccines have remained stable over time (between 2001, 2004 and 2006) and are comparable to provincial vaccine coverage results. Likewise, vaccine coverage in older children did not fluctuate between 2004 and 2006. Vaccine coverage increased for the newer vaccines introduced since the implementation of the National Immunization Strategy thereby highlighting the impact of policy decisions on immunization coverage.

\section{P076}

\section{COSTING OF IMMUNIZATION PROGRAMS IN BRITISH COLUMBIA}

M Mclvor $^{1}, \mathrm{C}$ Thompson $^{2}$

${ }^{1}$ Vancouver, BC; ${ }^{2}$ Victoria, BC

BACKGROUND: There was inadequate province-wide acceptance of the BCCDC immunization costing model and the major assumptions.

PURPOSE: To develop a well understood, updatable and accepted costing model for immunization programs in BC by using a collaborative management approach.

METHODS: A project management strategy was used by a small working group which reported existing published and unpublished costing evidence, gained acceptance of overall guiding principles for costing of immunization programs, and organized in-depth interviews of stakeholders by objective third parties. The assumptions of existing models were reviewed, and accepted or refuted using external standards and negotiation.
RESULTS: A new accepted model was developed to determine costs of offering quality immunization services to $100 \%$ of the target population efficiently and safely. The model considers infant, school and mass immunization programs and accounts for costing variability of first versus subsequent doses in a series, and new vaccines at existing clinics versus new vaccines at new clinics. Staff travel, overhead and time for biologicals management are considered along with variable start-up costs including staff training, immunization promotion, and cold chain equipment. Vaccine administration staffing costs for public health nurses, managers and clerical staff were defined which included consent, immunization, documentation and follow-up at $\$ 6-15$ per dose.

CONCLUSION: The collaborative management approach assisted in achieving widespread acceptance of an updated costing model for immunization programs. Transparency regarding calculations and decision-making encouraged trust. Soliciting feedback from stakeholders highlighted the importance of including more than the price of vaccine when costing immunization programs. The approach ensured support from the beginning as participants were involved in the early stages of the project and represented a new model of governance with the BC Ministry of Healthy Living and Sport sharing the responsibility of developing a realistic and comprehensive immunization costing model.

\section{P077}

\section{REDUCING VACCINE WASTAGE IN BRITISH COLUMBIA M Mclvor, M Naus, B Gabel, C McIntyre, R Wong, J Seto, R Taylor, B Deeter Vancouver, BC}

BACKGROUND: Vaccine wastage in British Columbia (BC) has historically averaged around $6 \%$ based on value of non-usable vaccine compared to distributed vaccine, as assessed at the central level. Vaccine budgets have increased dramatically in the past decade, necessitating additional efforts at wastage reduction. Managing wastage is complex due to the division of responsibilities between central, regional, and end user levels. We undertook to reduce wastage through a comprehensive set of initiatives.

PURPOSE: To describe a province-wide process to decrease vaccine wastage to $3 \%$ or less.

METHODS: A collaborative management method was applied; multi-sectoral working groups were formed to facilitate strong buy-in with stakeholders. Consensus was achieved by objectively assessing, analyzing and quantifying actual wastage (in dollars and doses) using vaccine shipped data and cold chain incident and field return reports. Resulting work plans addressed three key themes: logistical issues, knowledge gaps, and communication and accountability. Feedback was provided to the stakeholders regularly. New logistics methods included shipping using vehicles with refrigerated storage and same-day delivery systems. Knowledge and accountability was increased by designating responsible staff in each public health office and communicating directly to them. Partnerships with vaccine manufacturers generated new evidence-based vaccine-specific stability information for use in cold chain incidents. Collaboration within BCCDC, with the regions and with the vaccine manufacturers was critical.

RESULTS: The wastage rate dropped from 6\% to 3.1\% from March 31, 2007 to March 31, 2010.

CONCLUSION: Vaccine wastage can be reduced significantly through improved vaccine transport infrastructure, less unnecessary loss of vaccines exposed to minor cold chain excursions, and improved knowledge and training of those who manage inventory as part of their job duties. Large-scale reductions in wastage were achieved in BC over a 3-year period through collaborative management between regional and provincial public health authorities and coordinated action with industry. 
P078

MAPPING THE UNIMMUNIZED: UNDERSTANDING VULNERABILITY TO VACCINE PREVENTABLE DISEASES IN CONSCIENTIOUS OBJECTORS

\section{S Chan, A Schneeberg, M Naus}

Vancouver, BC

BACKGROUND: Outbreaks of vaccine preventable diseases (VPDs) occur in unimmunized communities. When clustered, such communities are at continual risk from re-introduction of these diseases. Objectors who are interspersed among vaccinated individuals are also at higher risk of infection. A three part project was undertaken in British Columbia to describe, map, and understand conscientious objectors in BC. This abstract describes findings from part one.

PURPOSE: To conduct a literature review on vaccine refusing communities.

METHODS: Scientific journal database searches of PubMed and Google Scholar, as well as archive searches of ProMed, CCDR and MMWR were conducted. Search terms included: unimmunized, unvaccinated, vaccine exemption, vaccine refusal, orthodox, Waldorf, anthroposophic, conscientious objector, Dutch Reformed, independently or in combination with 'outbreak'. The search was limited to western world literature.

RESULTS: Twenty six papers (15 outbreak reports, 5 cross-sectional studies, 2 outbreak reports/retrospective cohort studies, 2 retrospective cohort studies, 1 case-control study, and 1 literature review) were retrieved. Four papers examined association between vaccination status and VPD risk. All found a risk between lack of vaccination and risk of contracting measles, rubella, and pertussis. Two papers analyzed characteristics of unvaccinated and vaccinated individuals, with opposing findings related to race, income, and household size. Among 17 outbreak reports, 15 involved religious exemptions and 16 reported vaccination status. Collectively, 88.6\% (4215/4759) of VPD cases were eligible for vaccines but unimmunized. Most cases were residents of the unimmunized community. One paper found a strong association between pertussis rates and geographic clustering of unvaccinated persons.

CONCLUSION: Published literature suggests a significantly higher risk of VPDs among unimmunized. Herd immunity provides greater protection to individual objectors within vaccinated communities but not to geographically clustered communities. No publications were found to enumerate and map unimmunized populations. Further analysis will be done in $\mathrm{BC}$ to locate and describe vulnerable groups and individuals.

\section{P079 \\ ADVERSE EVENTS FOLLOWING IMMUNIZATION IN CHILDHOOD - CHALLENGES FOR ASSIGNING CASE DEFINITION AND PROVIDING FOLLOW UP RECOMMENDATIONS}

\section{O Nnorom, V Dubey, K Beckermann}

Toronto, ON

BACKGROUND: Investigating an adverse event following immunization (AEFI) is part of routine public health practice. It is required for post-marketing surveillance to identify severe or rare events. Local health units collect information about an AEFI, determine if it meets the case definition and forward it to provincial and federal authorities. In the routine immunization schedule, children often receive multiple, concurrent vaccines. When an AEFI occurs, it can be difficult to determine which vaccine may be implicated and provide guidance on future immunization.

PURPOSE: To analyze and discuss complex adverse events following immunization in children.

METHODS: A chart review was completed of two complex paediatric AEFI cases among all cases reported to Toronto Public Health from January 2009-May 2010.
RESULTS: (1) A hypotonic-hyporesponsive episode occurred in a 2-month old boy who developed pallor, diaphoresis, limpness and reduced consciousness five minutes post co-immunization with Pediacel $\AA$ and Synflorix ${ }^{\circledR}$.

(2) An episode of thrombocytopenia occurred in a 12-month old boy who developed a purpuric rash, and subsequent platelet count of $3 \times 10^{9} \mathrm{~L}-1,21$ days post co-immunization with MMR and Menjugate ${ }^{\circledR}$ and 19 days post-Arepanrix ${ }^{\circledR}$. In both cases, the children received all vaccines for the first time. No other cause for the episodes was identified and the case definition was met. Recommendations for future vaccination were left to the clinician and/or local health unit.

CONCLUSION: Decisions regarding future immunization are not always clear as clinicians/local health units have limited experience with complex cases. Case definitions do not address causality which may be appropriate for surveillance purposes. However, at the individual client level, case definitions have different implications. This is especially true for AEFIs occurring from routine childhood immunizations where it is often not clear which vaccine could have caused the AEFI. Finally, federal decisions about severe or unusual AEFIs and determination of causality are rarely communicated back to the local health unit.

\section{P080}

COST-EFFECTIVENESS OF 13-VALENT PNEUMOCOCCAL CONJUGATE VACCINE COMPARED WITH 10-VALENT VACCINE IN PREVENTING PNEUMOCOCCAL INFECTIONS IN QUEBEC

J Roussy $^{1}$, H Kwan ${ }^{1}$, S Earnshaw ${ }^{2}$, R Farkouh ${ }^{2}$, S Hwang ${ }^{3}$, D Strutton ${ }^{3}$

${ }^{1}$ Kirkland, QC; ${ }^{2}$ Research Triangle Park, NC; ${ }^{3}$ Collegeville, PA

BACKGROUND: Streptococcus pneumoniae (Sp) is responsible for invasive (meningitis, bacteremia) and non-invasive (pneumonia, acute otitis media $(\mathrm{AOM})$ ) pneumococcal infections. Thirteen-valent pneumococcal conjugate vaccine (PCV13) and 10-valent pneumococcal conjugate vaccine (PCV10) are two vaccines currently used to prevent these infections. PCV13 offers broader protection against $\mathrm{Sp}$ by three additional serotypes; 3, 6A, and 19A. In this analysis, we assess the cost-effectiveness of PCV13 vs. PCV10.

METHODS: A decision-analytic model was developed to examine the impact of each vaccine over one year. For vaccinated individuals, direct effects were applied. For individuals not vaccinated, indirect effects were simulated. Direct and indirect effects were derived from 7 -valent pneumococcal vaccine. Additional direct effect of $4 \%$ was attributed to PCV10 for moderate to severe AOM to account for potential non-typable Haemophilus influenzae benefit. Disease incidence, serotype coverage, percentage of individuals vaccinated, and costs (2009 CAN\$) were obtained from local sources.

RESULTS: In Quebec, use of PCV13 may prevent 5,240 additional cases of pneumococcal infections vs. PCV10. Of these, 19 are meningitis and 292 are bacteremia. PCV13 may also prevent 21 deaths and improve quality-adjusted life years by 397 . When considering costs of treating infections and vaccination programs, PCV13 generates savings of $\$ 1,356,638$. As a result, PCV13 dominates (i.e., more effective, less costly) PCV10. For each child vaccinated with PCV13, the health care system would save $\$ 20$. One-way sensitivity analyses showed PCV13 to always be dominant or cost-effective vs. PCV10.

CONCLUSIONS: Considering the epidemiology of pneumococcal disease in Quebec, PCV13 is shown to be the optimal vaccine as it provides substantial public health and economic benefits relative to PCV10. 
P081

ONTARIO'S SCHOOL-BASED HPV VACCINATION PROGRAM: SCHOOL BOARD ASSENT AND PARENTAL CONSENT

SE Wilson, SL Deeks, E Karas, M Simpson, B Henning,

E Bontovics, NS Crowcroft

Toronto, ON

BACKGROUND: In 2007, Ontario introduced a publicly funded school-based HPV immunization program for grade 8 girls using Gardasil®. Prior to implementation, the Ontario Conference of Catholic Bishops released a statement regarding HPV vaccination in Catholic schools. There was also a 'black-out' on promotion of provincial programs due to an upcoming election. Although there is no minimum age for consent to treatment in Ontario, traditionally schools require parental consent and parents expect to consent for childhood immunizations.

PURPOSE: To document experiences of Ontario's Health Units (HUs) in obtaining agreement from school boards for vaccine delivery in schools and to review procedures used to obtain informed consent for the program.

METHODS: Immunization program managers at Ontario's $36 \mathrm{HUs}$ were invited to participate in a semi-structured questionnaire as part of a process evaluation of the school-based HPV vaccination program. Qualitative data from interviews were categorized using thematic content analysis.

RESULTS: All 36 HUs participated in the evaluation; 16 (44\%) reported difficulties receiving agreement from local school board(s) to administer HPV vaccine in schools. Two Catholic school boards have not permitted HPV vaccine clinics in their schools: 1 only during the first year of the program and another since the fall of 2008. In terms of consent to receive HPV vaccine, all HUs request parental consent and 5/36 also request or encourage student consent; 14 HUs indicated they would immunize a grade 8 girl at a school clinic, in the absence of parental consent, if the student requested immunization and was judged capable of providing informed consent.

CONCLUSIONS: Many HUs reported challenges in receiving support from local school boards. Despite this, vaccine clinics have been offered in all but 2 public school boards since 2007. All HUs request parental consent before HPV immunization at school-based clinics; $39 \%$ consider immunizing in the absence of parental consent.

\section{P152}

\section{UNDERSTANDING IMMUNIZATION - AN IMMUNIZATION} DECISION MAKING TOOLKIT FOR FIRST NATIONS PARENTS

\section{JM O'Neill}

\section{Ottawa, ON}

BACKGROUND: The First Nations Centre (FNC), National Aboriginal Health Organization (NAHO) has created a decision making toolkit to help First Nations parents understand immunizations. The purpose of the toolkit is to help on-reserve health care professionals provide parents with information about vaccines based on science and research to help make informed decisions regarding immunization.

This toolkit is one in a series of health promotion toolkits developed by the FNC.

PURPOSE: This toolkit provides a wide array of vaccine and immunization information within many short factsheets, pamphlets, and booklets that each focus on only one topic. Each resource contained within the toolkit is First Nations focused.

METHODS: This toolkit consolidates accessible information about vaccines to support First Nations health. Topics included in the toolkit include: the immunization schedule; the diseases with routine immunization; the risks of the diseases; vaccine components; the immediate side effects of the vaccines; the clinical and immunological effectiveness; the potential longer term effects of vaccines; and other information including the lag time between vaccination and protection and herd immunity. Worksheets included in the toolkit provide questions for parents to consider throughout the decision making process. The toolkit addresses aspects of informed decision making including accurate knowledge, a sense of satisfaction and realities of the community environment.

All resources provide links to other information, programs, and services that may be helpful to the reader.

RESULTS: The toolkit will be distributed to First Nations communities and posted on the FNC website. The FNC will invite feedback from parents and healthcare providers on the resources within the toolkit. The toolkit will promote capacity building and assist in knowledge translation.

CONCLUSION: This toolkit fills an identified gap in immunization resources and is culturally appropriate for First Nations families.

\section{INFLUENZA}

\section{P082}

MASS IMMUNIZATION REGISTRY: NEWFOUNDLAND AND LABRADOR'S PANDEMIC SOLUTION

GA Butler, C OKeefe, K Butt, D Allison

St John's, NL

BACKGROUND: In June 2009 World Health Organization raised the level of influenza pandemic alert to Phase 6; provincial pandemic planners in Newfoundland and Labrador recognized that a robust system was needed for recording, tracking and reporting on the residents of the province of Newfoundland and Labrador who received the pH1N1 vaccine.

PURPOSE: The Mass Immunization Registry (MIR) a centralized electronic system was conceived to facilitate easy, real time and accurate collection of data which would allow for monitoring and evaluation of vaccine coverage. This system would also fulfill the requirements for weekly reporting to the Public Health Agency of Canada.

METHODS/PROJECT: The Office of the Chief Information Officer in consultation with subject experts from the Department of Health and Community Services and representation from four Regional Health Authorities (RHAs) identified a solution: a fully networked centralized, web-enabled computer system with a paper backup plan to allow for hundreds of clients to be processed daily. Once built the application was populated with data from the provinces central registry. The database allowed simple reporting.

RESULTS: In spite of time lines the MIR became a reality. The system allowed the province to track vaccine coverage and uptake by region. This helped direct and modify delivery strategies for managing the vaccination program. It helped focus the program during vaccine shortage on groups at highest risk for H1N1disease. The system provided a standard process for data collection, documentation and reporting. Tracking of vaccine lot numbers ensured safety issues where addressed. As well the system fulfilled the daily and weekly reporting that was required.

CONCLUSION: A standard approach to the collection of vaccination data at the point of delivery is important to provide vaccine coverage information. The success of Newfoundland and Labrador's MIR application helped this province achieve the best uptake of H1N1 vaccine across Canada. This project can be used as a template for other similar projects that fight vaccine preventable illness. 
P083

COVERAGE AND PREDICTORS OF INFLUENZA IMMUNIZATION AMONG ONTARIO CHILDREN AGED 6-23 MONTHS

\section{MA Campitelli, M Inoue, JC Kwong, A Guttmann \\ Toronto, ON}

BACKGROUND: Current estimates for influenza immunization coverage of children aged 6-23 in Ontario are based on telephone surveys which may be biased. Few studies have described predictors of influenza immunization in this age group and, specifically, for low-birth weight infants.

PURPOSE: To determine influenza immunization coverage for children 6-23 months old in Ontario from 2002-2008 and to describe predictors of vaccination.

METHODS: Using hospital discharge records, we identified all infants born alive in an Ontario hospital between April 1, 2002 and March 31, 2008. Immunization status was ascertained by linkage to Ontario physician billing claims data. Infants were categorized as fully, partially or not immunized depending on the number and timing of vaccines administered. A generalized linear mixed model was used to determine the association between immunization status and infant, physician and maternal characteristics, while accounting for the clustering of children within physicians.

RESULTS: Influenza immunization in children 6-23 months old was low for the first influenza season of the study period ( $1 \%$ fully immunized during the 2002-03 season), rose sharply in the following three years (7-9\% fully immunized during the 2003-04 to 2005-06 influenza seasons), but has steadily declined since (4-6\% fully immunized during the 2006-07 to 2008-09 influenza seasons). Maternal influenza immunization (Odds Ratio [OR] 4.34; 95\% Confidence Interval [CI] 4.24-4.44), having a mother less than 20 year at first childbirth (OR 0.70; $95 \%$ CI $0.66-0.75$ ), having a pediatrician as the primary care practitioner (OR $1.9195 \%$ CI 1.74-2.10) and number of primary care visits one year following birth (OR 1.08; 95\% CI 1.08-1.09) were all significantly associated with full immunization. These associations were similar for low birth-weight infants.

CONCLUSIONS: Influenza immunization coverage among children aged 6-23 months may not be as high as previously suggested. Interventions to improve coverage should target both physicians and families.

\section{P084}

PANDEMIC (H1N1) INFLUENZA IMMUNIZATION RESPONSE IN CANADIAN FEDERAL PENITENTIARIES: VACCINATION CAMPAIGN RESULTS

J Heys, J Smith, L Poliquin, $\underline{\mathrm{R} J o h n s o n}$, T Garrahan,

L Knox-Kinsman, A Hume

Ottawa, ON

BACKGROUND: Correctional Service Canada (CSC) provides health services to inmates incarcerated in federal correctional institutions. Between October, 2009 and April, 2010, pandemic (H1N1) influenza immunization campaigns were conducted in all 57 CSC institutions across Canada.

PURPOSE: To describe successes and challenges of the pandemic (H1N1) influenza vaccination campaign in the Canadian federal correctional system.

METHODS: A multi-disciplinary team was assembled to oversee the pandemic (H1N1) response in CSC. A reporting template was developed to track immunizations and vaccination campaign status initially on a daily basis and then on a weekly/monthly basis. Immunizations were offered to offenders based upon the Public Health Agency of Canada priority sequencing criteria.

RESULTS: Campaign results are shown in Table 1. In the first priority sequence 4660 inmates (33.5\% of the inmate population) were identified and 3932 offenders were immunized (estimated priority vaccination campaign coverage $84.4 \%$ ). In total 8120 inmates were immunized in CSC (estimated overall coverage 55.7\%).

To optimize uptake, active immunization campaigns were conducted where possible and health promotion materials, including inmatecreated posters based on key messaging, were distributed throughout the institutions. Challenges included applying priority sequencing criteria in the federal correctional environment and delivering a consistent national inmate immunization program.

Table 1

\begin{tabular}{|c|c|c|c|c|c|c|}
\hline \multirow[b]{3}{*}{$\begin{array}{l}\text { CSC } \\
\text { Region }\end{array}$} & \multicolumn{3}{|c|}{ Priority Vaccination Campaign } & \multicolumn{3}{|c|}{$\begin{array}{c}\text { Priority + Mass Vaccination } \\
\text { Campaign }\end{array}$} \\
\hline & & & Estimated \% & & & Estimated \% \\
\hline & $\begin{array}{l}\text { \#Priority } \\
\text { Inmates } \\
\text { Identified }\end{array}$ & $\begin{array}{l}\text { \# Inmates } \\
\text { Immunized }\end{array}$ & $\begin{array}{c}\text { Priority } \\
\text { Inmates } \\
\text { Immunized }\end{array}$ & $\begin{array}{l}\text { Cumulative } \\
\text { Inmate } \\
\text { Population }\end{array}$ & $\begin{array}{c}\text { Total \# } \\
\text { Inmates } \\
\text { Immunized }\end{array}$ & $\begin{array}{c}\text { Inmate } \\
\text { Population } \\
\text { Immunized }\end{array}$ \\
\hline Atlantic & 596 & 443 & $74.3 \%$ & 1433 & 1101 & $76.8 \%$ \\
\hline Quebec & 923 & 806 & $87.3 \%$ & 3388 & 2080 & $61.4 \%$ \\
\hline Ontario & 1324 & 1046 & $79.0 \%$ & 3964 & 1612 & $40.7 \%$ \\
\hline Prairie & 1147 & 1027 & $89.5 \%$ & 3787 & 2268 & $59.9 \%$ \\
\hline Pacific & 670 & 610 & $91.0 \%$ & 2015 & 1059 & $52.6 \%$ \\
\hline NATIONAL & 4660 & 3932 & $84.4 \%$ & 14587 & 8120 & $55.7 \%$ \\
\hline
\end{tabular}

CONCLUSIONS: Several challenges were overcome and CSC immunized more than half of all inmates for pandemic (H1N1) influenza in a short timeframe.

\section{P085}

\section{ESTIMATING BACKGROUND RATES OF GUILLAIN-BARRÉ SYNDROME TO ASSIST WITH EVALUATING SAFETY CONCERNS DURING A MASS PANDEMIC IMMUNIZATION CAMPAIGN IN ONTARIO}

GH Lim ${ }^{1}$, SL Deeks' ${ }^{1}$ M Simpson ${ }^{1}$, C Mackie ${ }^{2}$, L Rosella ${ }^{1}$, C Achonu $^{1}$, B Henning ${ }^{1}$, N Crowcroft ${ }^{1}$

${ }^{1}$ Toronto, ON; ${ }^{2}$ Hamilton, ON

BACKGROUND: The province of Ontario (population 12.8 million) initiated mass immunization clinics with adjuvanted pandemic H1N1 influenza vaccine in October 2009. Due to the campaign size, temporal associations between Guillain-Barré syndrome (GBS) and vaccine were expected. However, GBS is also associated with influenza illness.

PURPOSE: To estimate the number of background GBS cases expected to occur within the estimated vaccinated population of Ontario and the number of cases expected in association with influenza infection.

METHODS: Expected baseline incidence rates of GBS were determined from published Canadian studies, and ranged from 0.97 to 2.32 cases per 100,000 people per year. Ontario demographic data were obtained from Statistics Canada. Vaccine coverage data were projected using the number of doses distributed by the province and early coverage data reported to the province by local health units. The expected number of cases was derived by applying incidence rates to the estimated vaccinated population. The number of GBS cases expected to occur as a result of influenza infection during the vaccination campaign was determined based on risk estimates of GBS after influenza infection and provincial influenza infection rates.

RESULTS: We estimated that approximately 5.1 million Ontarians were vaccinated by December 27 (40\% of the population). Between 6 and 14 coincident background cases of GBS could be expected to occur among individuals vaccinated by the 9th week of the vaccination campaign; 5 cases were reported. During the same time period between 9 and 22 additional cases would have been expected to be associated with influenza illness.

CONCLUSIONS: Awareness of background rates of GBS is critical when assessing adverse events following influenza immunisation. These data provided reassurance when a GBS case was observed during the 
campaign. Although GBS is associated with influenza illness, perceptions of GBS risk associated with vaccine dominate in the media and scientific literature.

\section{P086 \\ MOVING UP, MOVING DOWN, COMMUNICATIONS FLOW TO AND FROM MASS IMMUNIZATION CLINICS}

\section{A Mathur, K Beckermann \\ Toronto, ON}

BACKGROUND: During the 2009 Influenza Pandemic, Toronto Public Health (TPH) was mandated to deliver pandemic H1N1 vaccine to all Torontonians efficiently. The death of a previously healthy, 12 year old male hockey player received intense media attention. This led to heightened public fear, demand and urgency to open clinics earlier. Information about the vaccine and priority groups was constantly changing. It was important to ensure that the new and frequently changing information was received, understood and implemented by clinic staff in a timely manner.

PURPOSE: To discuss communication logistics and lessons learned in mass vaccination clinics when multiple staff and sites are involved.

METHODS: A restrospective analysis of communication methods used for mass vaccination clinics by TPH.

RESULTS: Approximately 560 TPH staff and over 200 agency nurses operated the ten large clinics where over 231,000 clients were immunized. The Incident Management System (IMS) was used for the response enlisting an operations lead for clinics. Communication was carried out through teleconferences, e-mails and individual phone consultations.

Throughout the outbreak, communication strategies were frequently adjusted since each clinic was unique requiring different communication solutions as the message was constantly changing.

Additional communication support was needed to reduce staff concerns and ensure safe operations. This was achieved through adding management support, increasing teleconference frequency, expanding teleconference participants, providing on-site expert support and adjusting electronic communications. The logistics team in the IMS structure implemented the communication messages.

CONCLUSION:

- Expert leadership, highly skilled and trained clinic staff are essential.

- Listen and respond quickly to clinic concerns and logistics.

- Recognize that one solution will not meet all needs.

- Ensure strong, adequately resourced and supported operations team with vaccine expertise.

- Train early, train often, and train for clinic realities.

- Debrief following the event.

- Incorporate learnings into future plans.

\section{P087 \\ EFFICACY AND SAFETY OF PANDEMIC INFLUENZA VACCINE IN PREGNANCY \\ Y Guo $^{1}$, V Allen ${ }^{2}$, E Bujold ${ }^{3}$, B Coleman ${ }^{1}$, S Drews ${ }^{4,5}$, K Gouin ${ }^{3}$, L Hendson $^{5}$, J Johnston ${ }^{6}$, KC Katz ${ }^{1}$, BE Lee $^{5}$, M Levy $^{5}$, M Loeb $^{6}$, M Louie ${ }^{4,5}$, S Manos ${ }^{2}$, M McArthur ${ }^{1}$, C McCourt ${ }^{7}$, A McGeer ${ }^{1}$, S McNeil'2, D Money ${ }^{8}$, K Murphy ${ }^{1}$, L Pelletier ${ }^{7}$, R Rodin $^{7}$, R Sauve $^{4}$, G Taylor ${ }^{5}$, A Ugnat ${ }^{7}$, D Wilson ${ }^{4}$, G Zahariadis ${ }^{5}$ ${ }^{1}$ Toronto, ON; ${ }^{2}$ Halifax, NS; ${ }^{3}$ Quebec, QC; ${ }^{4}$ Calgary, AB; ${ }^{5}$ Edmonton, $\mathrm{AB} ;{ }^{6} \mathrm{Hamilton}, \mathrm{ON} ;{ }^{7}$ Ottawa, $\mathrm{ON} ;{ }^{8}$ Vancouver, $\mathrm{BC}$ BACKGROUND: There is insufficient evidence for the safety and efficacy of seasonal and pandemic influenza vaccines in pregnancy. PURPOSE: To evaluate the efficacy and safety of seasonal and 2009 pandemic H1N1 vaccine in pregnant women, we conducted a multi- center prospective cohort study. \\ METHODS: Participants were recruited from 8 sites across Canada from $9 / 2009$ to $1 / 2010$. Demographic characteristics, influenza}

vaccination status, potential risk factors for congenital abnormalities and birth outcomes were collected. Nasal swab specimens were collected from participants with acute respiratory illness (ARI).

RESULTS: 875 participants were enrolled. Median age was $33 \mathrm{yrs}$ (range 18-50) and median gestation at enrolment $20 \mathrm{wks}$ (range 5-35). $33 \%$ (293) participants were pregnant for the first time, 50\% (443) had high risk pregnancies, 12\% (102) were asthmatic, 7\% (63) were diabetic. 256 (29\%) received 2009 seasonal flu vaccine, 550 (62\%) 2009 pH1N1 vaccine. Thirteen (1.5\%) women developed symptomatic pH1N1 infection, 4 before H1N1 vaccine was available. After vaccine was available, $4 / 550(0.7 \%)$ vaccinated and 5/325 (1.5\%) unvaccinated women developed $\mathrm{pH} 1 \mathrm{~N} 1$ ( $\mathrm{VE}=42 \%, 95 \% \mathrm{CI}-47-84 \%, \mathrm{P}=.5) .3 / 4$ infections in vaccinees occurred within 5 days of vaccination. Comparing pH1N1 vaccinated and unvaccinated women, there was no difference in fetal loss $(7 / 550,1.3 \%$ vaccinees $\mathrm{v} 11 / 325,3.3 \%$ unvaccinated, $\mathrm{P}=.06)$ or premature birth $(31 / 359(8.6 \%)$ vaccinated $\mathrm{v} 23 / 185$ $(12 \%)$ unvaccinated $\mathrm{P}=.21)$. Similar results were obtained for seasonal flu vaccine: fetal loss: 4/256 vaccinated $(1.5 \%)$ vs 14/619 unvaccinated $(2.2 \%), p=.53$, premature delivery: $13 / 168(7.8 \%)$ vaccinated vs $41 / 376(11 \%)$ unvaccinated, $p=.25$. Of 261 vaccinees reporting weekly data, $11(4.2 \%)$ reported an adverse event requiring missed work or an MD visit within 7 days of vaccination, most commonly acute respiratory illness $(\mathrm{N}=7)$. Only one event (arm numbness) was thought to be vaccine related. No serious adverse events were reported.

CONCLUSIONS: Vaccine became available too late, and the attack rate was too low, to assess vaccine efficacy in this cohort. Results to date suggest that both seasonal and pandemic vaccines were safe.

\section{P088}

SEASONAL AND PANDEMIC INFLUENZA VACCINE UPTAKE IN CANADA: A NATIONWIDE SURVEY OF ATTITUDES AND BEHAVIOURS OF CANADIAN ADULTS

M Kiberd ${ }^{1}$, J Slaunwhite ${ }^{1}$, K Slayter ${ }^{1}$, S Halperin ${ }^{1}$, B Halperin ${ }^{1}$, S Bowles ${ }^{1}$, V Gilca ${ }^{2}$, C Sauvageau ${ }^{2}$, E Dube ${ }^{2}$, N Boulianne ${ }^{2}$,

F Boucher $^{2}$, F Lavoie $^{2}$, M Ouakki ${ }^{2}$, I Gemmill ${ }^{3}$, SA McNeil ${ }^{1}$ ${ }^{1}$ Halifax, NS; ${ }^{2}$ Quebec, QC; ${ }^{3}$ Kingston, ON

BACKGROUND: pH1N1 influenza vaccine (pH1N1V) and seasonal influenza vaccine (SIV) are recommended for all Canadians. Implementation of immunization programs is a provincial responsibility and varies across Canada. National and provincial uptake rates and program factors impacting uptake are not yet known. It is unclear how pH1N1V recommendations will impact the uptake of SIV.

METHODS: We conducted a nationwide web-based survey exploring attitudes \& behaviours of Canadian adults regarding pH1N1V and SIV. Differences between provinces and by age were explored. Results are weighted by gender, age, region, language, and education to achieve a representative sample.

RESULTS: 4,067 adults responded; $47.5 \%$ were $18-44$ y, 35\% 45-64y $\& 17.5 \% 65+y$. All provinces were represented. 38.9\% (26.5-41.5) reported receipt of SIV in 2008/09. 24.4\% had received SIV by the end of December 2009; an additional $17.1 \%$ reported intent to receive the SIV this season. $42.2 \%$ had received $\mathrm{pH} 1 \mathrm{~N} 1 \mathrm{~V}$. Uptake of $\mathrm{pH} 1 \mathrm{~N} 1 \mathrm{~V}$ varied by province from $34 \%$ to $66.1 \%$. Only $54 \%$ of Canadians felt they were clear about which vaccines they needed. $60 \%$ of Canadians felt that SIV is safe compared to only $46.3 \%$ who felt pH1N1V was safe. $43 \%$ felt that influenza adversely impacts health and $58.3 \%$ thought that SIV is effective in preventing influenza. 93.3\% disagreed that SIV increases the risk of being infected with pH1N1 influenza.

DISCUSSION: If all those intending to receive the seasonal influenza vaccine actually do, national coverage would only be $41.5 \%$ for SIV, roughly equal to this year's pandemic coverage. However, this estimate is contingent on all those intending to receiving the vaccine actually receiving it, which is improbable. These early estimates would suggest that SIV coverage will likely be the same or less compared to last year's SIV coverage. Thus, it would appear that there was not a strong interaction between seasonal influenza and pandemic influenza (positive or 
negative). Also, the media coverage of the potential for receipt of SIV to increase risk of $\mathrm{pH} 1 \mathrm{~N} 1$ infection did not seem to strongly impact public opinion.

\section{P089 \\ A COST COMPARISON OF ELECTRONIC AND HYBRID DATA COLLECTION SYSTEMS IN ONTARIO DURING PANDEMIC AND SEASONAL INFLUENZA VACCINATION CAMPAIGNS \\ JA Pereira $^{1}$, J Foisy ${ }^{1}$, JC Kwong ${ }^{1}$, C Heidebrecht ${ }^{1}$, S Quach ${ }^{1}$, S Quan¹, M Guay², B Sander1 \\ ${ }^{1}$ Toronto, ON; ${ }^{2}$ Sherbrooke, QC}

BACKGROUND: During the 2009 H1N1 vaccination campaign, 30 of Ontario's 36 public health units (PHUs) collected client-level immunization data using an electronic system, while the remainder used hybrid systems (comprising paper-based and electronic components). Although cost has been identified as a barrier for implementing electronic systems, a system cost comparison has not been previously conducted.

OBJECTIVES: To compare projected Year 1 (pandemic), Year 2 and Year 5 (regular influenza seasons) costs associated with implementing hybrid and electronic systems in PHUs of population sizes 100,000, 500,000 and $1,000,000$.

METHODS: A convenience sample of six Ontario PHUs provided itemized costs of equipment and staffing during the H1N1 campaign and staffing algorithms for seasonal campaigns. We standardized resources at a population level, and assumed replacement of electronic equipment at Year 5 and public health vaccine administration rates of $18 \%$ and $2.5 \%$ for $\mathrm{H} 1 \mathrm{~N} 1$ and seasonal campaigns, respectively. Year 2 and 5 costs were discounted at $5 \%$.

RESULTS: Compared to a consistent hybrid system cost per capita of $\$ 2.83$ in Year 1, electronic system costs decrease as population size increases ( $\$ 2.92$ for $100,000, \$ 2.77$ for 500,000 and $\$ 2.72$ for $1,000,000$ ) due to capped data collection software fees. In Year 2, hybrid vs. electronic system costs per capita are $\$ 0.24$ vs. $\$ 0.23$ while in Year 5 (partial equipment replacement for seasonal clinics), hybrid system costs are $70 \%$ of electronic system costs. Of total costs, staffing comprised $>90 \%$ for hybrid systems and $50-75 \%$ for electronic systems. Sensitivity analyses identified that factors including higher nurse wages, increased nursing hours and lower laptop prices minimized between-system cost differences.

CONCLUSION: Given that staffing rather than equipment is the major contributing cost of data collection systems, the cost differential between hybrid and electronic systems was not substantial. The electronic system's intangible benefit is having data rapidly available for reporting.

\section{P090 \\ A POTENT CELLULAR IMMUNE RESPONSE IN THE PRESENCE OF PROTECTIVE ANTIBODY LEVELS CORRELATES WITH BETTER PROTECTION AGAINST 1918 INFLUENZA IN FERRETS \\ $\underline{\text { S Pillet }}{ }^{1}$, D Kobasa ${ }^{1}$, I Meunier ${ }^{2}$, M Gray ${ }^{1}$, D Laddy ${ }^{3}$, DB Weiner ${ }^{3}$, V von Messling ${ }^{2}$, GP Kobinger ${ }^{1}$ \\ ${ }^{1}$ Winnipeg, MB; ${ }^{2}$ Laval, QC; ${ }^{3}$ Philadelphia, PA}

BACKGROUND: The identification of immune correlates of protection against highly pathogenic human-adapted influenza is instrumental in the development of the next generation of vaccines. Ferrets are widely used to model highly pathogenic human-adapted influenza infection and to evaluate influenza vaccine efficacy, but their use for host response assessment is limited by the lack of speciesspecific assays.

PURPOSE: Contribute to the identification of immune makers correlating with protective immunity against influenza infection.
METHODS: A detailed analysis of humoral and cellular immune responses was performed in ferrets immunized with DNA vaccine alone or in combination with a replication-incompetent adenovirus vector both expressing the hemagglutinin of the A/South Carolina/1/18 H1N1 (H1N1-1918) influenza A virus, and compared to the humoral and cellular immune responses elicited by a formalin-inactivated vaccine containing the hemagglutinin and the neuraminidase from the H1N1-1918 virus in the genetic background of the A/Puerto Rico/8/34 influenza A virus.

RESULTS: We report the development and validation of IFN- $\gamma$ ELISpot and cytometry assays, and their use for monitoring activated T-cells in ferrets. The several vaccine strategies stimulating different levels of immunity were selected and evaluated to identify patterns in cellular response correlating with protection against morbidity/mortality. In the presence of an antibody response within the protective range, the extent of the $\mathrm{T}$ cell response correlates with reduced morbidity in vaccinated ferrets infected with H1N1-1918 influenza virus.

CONCLUSION: This study highlights the role of the T-cell response as an additional marker to more accurately define immune protection against influenza virus in this highly relevant animal model.

\section{P091}

SENSORY DISTURBANCES FOLLOWING ADMINISTRATION OF AS03-ADJUVANTED PANDEMIC H1N1 VACCINE: SURVEILLANCE SUMMARY FROM QUEBEC, CANADA I Rouleau ${ }^{1}$, G De Serres ${ }^{1}$, M Benoît ${ }^{1}$, E Toth ${ }^{1}$, S Ménard ${ }^{1}$, M Landry $^{1}$, J Grenier ${ }^{1}$, G Trudeau ${ }^{1}$, M Tremblay ${ }^{1}$, R Roussel ${ }^{1}$, S Giroux ${ }^{1}$, DM Skowronski²

${ }^{1}$ Quebec, QC; ${ }^{2}$ Vancouver, BC

BACKGROUND: In Quebec, 4.8 million people received a monovalent AS03-adjuvanted vaccine domestically produced (Arepanrix ${ }^{\mathrm{TM}}$; GlaxoSmithKline). We describe unusual reports of sensory disturbances notified to the passive surveillance system in Quebec after pH1N1 immunization.

MATERIALS AND METHODS: In Quebec, reporting adverse events following immunization (AEFI) is mandatory for physicians and nurses. Descriptions of reported adverse events consistent with sensory disturbances (anesthesia, paresthesia, numbness, prickling, pins \& needles or pain) that were not otherwise attributed to Guillain Barré Syndrome were analyzed and reviewed.

RESULTS: Provincially, 328 reports of sensory disturbances were received (6.8 per 100,000 doses), most of which came from immunized women (82\%), yielding a relative risk of 3.9 (CI95\% 2.9-5.1) compared to men. Most often reported were paresthesias described as numbness (85\%), prickling sensations or pins and needles $(20 \%)$ and dysesthesias described as painful (27\%) or burning sensations $(8 \%)$. Areas most often involved were: the immunized arm (68\%), a lower limb $(38 \%)$, or the face (34\%) (non mutually exclusive), but these sensations were not restricted to defined dermatomes. Clinical presentations were widely variable, $54 \%$ cases involved more than one area. For the remainder, symptoms were limited to the immunized arm $(23 \%)$, face $(15 \%)$, controlateral arm $(2 \%)$ or leg $(3 \%)$ or ipsilateral leg (2\%). Mean delay to onset was 26 hours after vaccination. Symptoms resolved within two days among $25 \%$ of patients and within 7 days among $73 \%$. One in three patients reported a chronic condition and $42 \%$ were taking medication at the time of vaccination.

CONCLUSIONS: We detected a signal of sensory disturbances associated with AS03-adjuvanted $\mathrm{pH} 1 \mathrm{~N} 1$ vaccine administered in 2009-2010. They came disproportionately from immunized women and were mostly transient. While symptoms may not correspond to known neurological diagnoses, this large case series flags a surveillance signal warranting further consideration with respect to causality and investigation of risk factors. 
P092

OVERVIEW OF THE IMMUNOGENICITY AND SAFETY OF THE FIRST INTRADERMAL INFLUENZA VACCINE

M Denis ${ }^{1}$, F Weber ${ }^{1}$, R Arnou ${ }^{2}$, A Ambrozaitis ${ }^{3}$, J De Juanes Pardo 4 ,

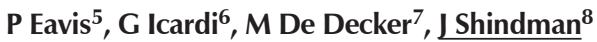

${ }^{1}$ Lyon, France; ${ }^{2}$ Angers, France; ${ }^{3}$ Vilnius, Lithuania; ${ }^{4}$ Madrid, Spain; ${ }^{5}$ Bath, United Kingdom; ${ }^{6}$ Genoa, Italy; ${ }^{7}$ Antwerp,

Belgium; ${ }^{8}$ Toronto, ON

BACKGROUND: Given the immune potential of the skin, an inactivated split-virion seasonal influenza vaccine was developed using the BD Soluvia ${ }^{\mathrm{TM}}$ a microinjection system (Becton Dickinson) to provide an easy to use, minimally invasive intradermal (ID) vaccination for adults.

PURPOSE: Two studies assessed the immunogenicity and safety of an intradermal influenza vaccine (INTANZA ${ }^{\mathrm{TM}}$, sanofi pasteur) using a microinjection system.

METHODS: Two randomized, controlled, multicentre phase 3 trials compared the safety and immunogenicity of ID influenza vaccination [Study 1: $9 \mu$ g haemagglutinin antigens (HA)/strain for adults aged 1859 years; Study 2: $15 \mu \mathrm{g} \mathrm{HA} /$ strain for older adults $\geq 60$ years] with intramuscular (IM) influenza vaccine (Vaxigrip ${ }^{\circledR} ; 15 \mu \mathrm{g} \mathrm{HA} /$ strain).

RESULTS: Study 1: 2249 adult subjects were vaccinated with ID $(\mathrm{N}=1796)$ or IM $(\mathrm{N}=453)$. The vaccines were equally immunogenic against all 3 strains with respect to seroprotection rates (titre $\geq 40$ ), post-/pre-vaccination geometric mean titre ratios (GMTRs) and seroconversion rates.

Study 2: 3701 older adult subjects were vaccinated with ID $(n=2612)$ or IM $(n=1089)$. The immune response for the ID vaccine was significantly higher for all 3 strains based on seroprotection rates, post- 1 pre-GMTRs, and seroconversion rates.

As expected with intradermal injection, minor transient visible injection site reactions tended to be more common with ID. Otherwise, the safety profiles of ID and IM vaccines were similar, with reactions typically mild, starting within 3 days of vaccination, and lasting for 3 days or less.

CONCLUSIONS: Using an easy-to-use and reliable microinjection system, ID influenza vaccine resulted in equivalent immunogenicity for adults $<60$ compared to a lower antigen dose, and superior immunogenicity in older adults compared to IM vaccination with an equivalent antigen dose. No new or unexpected safety concerns were identified.

\section{P093 \\ A SURVEY OF INDIVIDUAL PATIENT WILLINGNESS FOR SELF-REGISTRATION AND SELF-IMMUNIZATION IN MASS VACCINATION CLINICS IN ALBERTA, CANADA}

\section{Sikora, B McKim, M Johnson, T PCIRN Investigators}

\section{Edmonton, $\mathrm{AB}$}

BACKGROUND: A province-wide influenza immunization campaign was made available to the approximately 3.5 million inhabitants of Alberta, Canada in the winter of 2009. The campaign was organized and delivered by a single health administration system. Increasing the efficiency of such large-scale publicly-administered clinics is important to both improving the coverage of vaccines in the population and decreasing overall costs. Studies have demonstrated the majority of staff time is used in the registration and immunization stages of the clinic.

PURPOSE: To identify individual patient willingness to take an increased role in the immunization clinic processes of (1) registration and (2) immunization.

METHODS: A convenience sample of clinic sites was chosen for administration of a patient satisfaction survey between December 7 and 18, 2009. At each clinic site, patients were invited to complete the survey, following vaccination while waiting in the observation area of the clinic. The anonymous survey identified demographic information and patient views to questions on: previous use of self-registration for services, willingness to self-register for future vaccine clinics and interest in self-vaccination. Categories for these responses were pre-identified by consensus by the investigators.

RESULTS: 1601 patient surveys were returned from 8 different clinic sites. Approximately $43 \%$ of respondents had used self-registration processes in the past. Approximately $82 \%$ indicated they would be willing to self-register for a future vaccination clinic. Of all respondents, $35 \%$ stated willingness to self-administer the influenza vaccine, with $31 \%$ only if there was no needle involved.

CONCLUSION: This study identified that many patients attending mass vaccination clinics have used self-registration processes in the past and are willing to use self-registration for future influenza clinics. There was also a demonstrated willingness to self-immunize, but the current needle delivery mechanism may be a major barrier. This study identified areas for improving vaccination clinic flow, efficiency and ultimately vaccine coverage in a population.

\section{P094}

ÉVOLUTION MOLÉCULAIRE ET ANTIGÉNIQUE DES SOUCHES VIRALES HUMAINES D'INFLUENZA A/H3N2

\section{JAnn, M Hamelin, G Boivin}

\section{Québec, QC}

CONTEXTE : Les épidémies d'influenza constituent un problème majeur de santé publique. Elles sont provoquées par l'accumulation de mutations au niveau des acides aminés des sites antigéniques de la glycoprotéine hémagglutinine (HA) aboutissant à des dérives antigéniques, résultant parfois dans l'inadéquation des souches vaccinales saisonnières sélectionnées.

OBJECTIF : Corréler les mutations dans HA et la variabilité antigénique au niveau de la récente dérive antigénique de $\mathrm{A} /$ Brisbane/10/2007 (H3N2) à A/Perth/16/2009 (H3N2).

MÉTHODES : Nous avons effectué une analyse phylogénétique (MEGA 4.0) des séquences du gène HA des échantillons cliniques d'influenza A/H3N2 humain isolés entre mars et avril 2009 et certaines séquences de GenBank. Ensuite nous avons réalisé la caractérisation antigénique par l'inhibition de l'hemagglutination (HAI) et la microneutralisation en se servant des sérums de furets (A/Uruguay/716/2007 (Brisbane-like) et A/Perth/16/2009).

RÉSULTATS : Après l'analyse phylogénétique, les souches cliniques semblent se différencier en trois clades principaux qui sont selon l'OMS les clades Brisbane/10/2007, Victoria/208/2009 et Perth/16/2009. Les souches des clades Perth/16/2009 et Victoria/208/2009 partagent les mêmes substitutions antigéniques K158N et N189K (site B). Ces substitutions aboutissent à une diminution du titre HAI d'au moins quatre fois avec le sérum Uruguay/716/2007 et une augmentation d'au moins quatre fois avec le sérum Perth/16/2009 pour les souches appartenant à ces clades. Les souches Victoria/208/2009 ont les substitutions T212A (site D) et S262N (site E). Les souches Perth/16 ont les substitutions E62K (site E) et N144K (site A). Les tests de microneutralisation sont encore en cours de réalisation.

CONCLUSION : Il semble que nous ayons assisté à une dérive antigénique rapide des virus A/H3N2 au printemps 2009, impliquant la substitution de quatre acides aminés (K158N, N189K, E62K, N144K) dans trois sites antigéniques (A, B et $\mathrm{E})$. La détection plus rapide de nouveaux variants $\mathrm{A} / \mathrm{H} 3 \mathrm{~N} 2$ devrait prédire l'efficacité ou non des vaccins et permettre la mise en place d'une stratégie antivirale plus efficace. 
P095

RANDOMIZED TRIAL OF THE IMPACT OF THE OTTAWA INFLUENZA DECISION AID ON HEALTHCARE PERSONNEL CONFIDENCE TO BE IMMUNIZED

LW Chambers ${ }^{1}$, AE McCarthy ${ }^{1}$, S McNeil ${ }^{2}$, J Puxty ${ }^{3}$, K Wilson ${ }^{1}$,

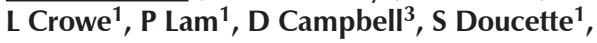

D Pierrynowski MacDougall ${ }^{2}$

${ }^{1} \mathrm{Ottawa}, \mathrm{ON}$; ${ }^{2} \mathrm{Halifax}, \mathrm{NS} ;{ }^{3}$ Kingston, ON

BACKGROUND: The Ottawa Influenza Decision Aid (OIDA)aims to assist healthcare personnel who are undecided about being immunized against influenza.

PURPOSE: Among healthcare personnel undecided about taking the vaccine, does use of the OIDA increase or decrease their confidence in their decision? Furthermore, does the OIDA affect the users' intent to be immunized?

METHODS: At six weeks into its influenza immunization campaign, healthcare personnel in a non-acute care hospital in January 2010 were randomized to receive standard influenza educational materials or these materials plus the OIDA through an interactive web-based session. In this hospital, all communication with personnel is conducted electronically, making this trial possible.

RESULTS: The site was accessed by 151 healthcare personnel but 107 completed all questions. Job types, gender and ages of the OIDA group were comparable to the non-OIDA group. 29\% (31 of 107) of participants at baseline reported being undecided about taking the vaccine. Undecided participants at baseline were less likely to be confident with their decision after reading the information than those planning to take or not take the vaccine $(p<0.03)$. Among the non-OIDA group, the baseline undecided became less confident after reviewing the information $(\mathrm{p}<0.03)$ However, in the OIDA group, the baseline undecided did not become any less confident than at baseline $(\mathrm{p}<0.5)$.

Also, the OIDA used on-line increased the percent intending to take the vaccine from $35 \%$ (17 of 48 ) to $45 \%$ ( 22 of 48 ) but in the nonOIDA group from $22 \%$ (13 of 59 ) to $27 \%$ (16 of 59 ) - $10 \%$ versus $5 \%$ $(\mathrm{p}<0.46)$

CONCLUSIONS: It appears that on-line vaccine information can lead to less confidence about whether or not to take the vaccine. However, when the information includes the OIDA this confidence is not affected. Also, completing the OIDA increased intent to take the vaccine more than not completing it. The H1N1 pandemic and delay and confusion in the 2009-2010 campaign may explain these results. Use of the OIDA group setting should be assessed in future.

\section{P096}

\section{WILLINGNESS TO PAY FOR VARIOUS CHARACTERISTICS OF INFLUENZA VACCINATION IN CANADIAN WORKING ADULTS}

A Chuck $^{1}$, P Jacobs ${ }^{1}$, P Murray ${ }^{2}$

${ }^{1}$ Edmonton, AB; ${ }^{2}$ Montreal, QC

BACKGROUND: Adult influenza vaccination has been shown to reduce absenteeism, physician visits, hospitalizations and mortality. Despite universal influenza vaccination programs less than half of adults (18-59 Years) choose to be vaccinated.

OBJECTIVES: This study assessed working adults $<60$ years of age preferences for influenza vaccination in terms of convenience and mode of administration, quantified using out-of-pocket willingness to pay (WTP).

METHODS: The study was an observational design that used a webbased discrete-choice survey to explore respondents' WTP. Thirty scenarios were presented to participants, differing in vaccine administration characteristics, convenience factors and out-of pocket cost expectation. Enhanced vaccine characteristics included the value of shorter needles and thimerosal free vaccines. Enhanced convenience factors included the value of earlier availability date, decreased waiting time and no appointment. Analysis was stratified based on participants' intention to be vaccinated and further, by offering all enhancements at no cost.

RESULTS: $50 \%$ ( $n=101 / 201)$ of respondents intended to be vaccinated at the start of the survey and were willing to pay $\$ 12.06$ for enhanced vaccine characteristics and $\$ 38.02$ for enhanced convenience factors.

Offering the enhanced scenarios at no cost resulted in 63\% ( $n=127 / 201)$ intending to be vaccinated (an additional 13\% ( $n=23 / 201)$ of respondents intending to be vaccinated). Overall, respondents not intending to be vaccinated significantly valued the vaccine characteristics (\$12.26) and enhanced convenience factors (\$34.89) compared with the non-enhanced scenario.

CONCLUSION: Enhanced vaccine characteristics and enhanced convenience factors resulted in an increased WTP for influenza vaccination. Addressing both factors also increased working adults' intention to be vaccinated when costs were removed, and therefore, may be utilized to increase vaccine uptake among working adults by public or employer influenza programs.

\section{P097}

\section{WILLINGNESS TO PAY FOR CHARACTERISTICS OF INFLUENZA VACCINATION IN NON-WORKING OLDER CANADIAN ADULTS}

\section{A Chuck ${ }^{1}$, P Jacobs ${ }^{1}$, P Murray ${ }^{2}$}

${ }^{1}$ Edmonton, $\mathrm{AB} ;{ }^{2}$ Montreal, QC

BACKGROUND: Influenza has the greatest impact with regards to morbidity and mortality in older adults. While vaccination rates are high $(71 \%)$ the efficacy of conventional intramuscular vaccination in preventing disease in older adults could be improved.

OBJECTIVES: This study assessed older non-working adults' ( $\geq 60$ years of age) preferences for influenza vaccination in terms of vaccine administration characteristics, convenience and mode of administration, quantified using out-of-pocket willingness to pay (WTP).

METHODS: The study was an observational design, using a web-based discrete-choice survey to explore respondents' WTP. Thirty scenarios were presented, differing in vaccine administration characteristics, convenience factors and out-of-pocket cost expectation. Enhanced vaccine characteristics included the value of shorter needles, higher immune response and thimerosal free vaccines. Enhanced convenience factors included the value of earlier availability date, decreased waiting time and no appointment. Analysis was stratified based on participants' intention to be vaccinated and further, by offering all enhancements at no cost.

RESULTS: $76 \%(n=157 / 206)$ of older adults intended to be vaccinated at the start of the survey, and were willing to pay $\$ 31.32$ for enhanced vaccine characteristics and $\$ 17.05$ for enhanced convenience factors. WTP costs were statistically significantly higher $(\mathrm{p}<0.05)$ than the nonenhanced scenario. Offering the enhanced scenarios at no cost resulted in $83 \%(n=173 / 206)$ intending to be vaccinated $(7 \%(n=16 / 206)$ more intending to be vaccinated). Individuals intending to be vaccinated with the enhanced scenario were willing to pay $\$ 40.45$ for enhanced vaccine characteristics and $\$ 23.18$ for enhanced convenience factors

CONCLUSION: Enhanced vaccine characteristics, including a higher immune response, and enhanced convenience factors resulted in an increased WTP for influenza vaccination. Older adults perceived significantly higher value for enhanced vaccine characteristics than enhanced convenience factors. Influenza vaccination programs for older adults may benefit from programs using vaccines that are thimerosal free, have smaller needles and have an increased immune response. 
P098

THREE YEAR ANALYSIS OF INFLUENZA VACCINE UPTAKE IN MCMASTER UNIVERSITY STUDENTS

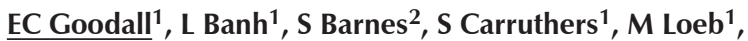

\section{Smieja ${ }^{1}$}

${ }^{1}$ Hamilton, ON; ${ }^{2}$ Toronto, ON

BACKGROUND: Vaccination is an effective way to reduce the risk of contracting influenza, and is universally recommended to children over age 6 months and to all adults in Ontario. Within the context of an influenza pandemic, university-aged students - especially those living in residence - may be particularly susceptible to respiratory outbreaks. Assessing the intent to get vaccinated and the actual uptake of available vaccination will provide important information for public health procedures during future pandemics.

PURPOSE: We assessed the intent to seek vaccination, and the actual uptake of seasonal flu vaccine over three separate years, and the intent to seek $\mathrm{H} 1 \mathrm{~N} 1$ vaccination in McMaster University students.

METHODS: During the 2007/8, 2008/9 and 2009/10 influenza seasons, single and serial cross-sectional surveys were self-administered by undergraduate students via classroom visits and survey stations across campus. 929 questionnaires were administered in 2007/8, 899 during 2008/9, and 1056 during 2009. A cohort of 116 students was recruited from students surveyed in 2009, and follow-through on vaccination intent was assessed by electronic questionnaires.

RESULTS: Intent to seek vaccination was similar in autumn 2007/8 and $2008 / 9$ when, respectively, $24.4 \%$ and $19.9 \%$ of students reported that they already had or were planning to receive the seasonal flu shot. In 2009/10, intent to seek H1N1 vaccination was higher (40.5\% intending, $36.2 \%$ unsure $)(\mathrm{p}<0.00)$. Interestingly, follow-through was equally low across all three influenza seasons ( $p=0.32$ ). In 2007/8, 14.2\% of students reported having received the seasonal flu vaccine, compared to $16.3 \%$ in $2008 / 9$, and $12.5 \%$ in 2009/10. Follow-through was greater for H1N1 (23.5\%) but this was not statistically significant.

CONCLUSIONS: Despite the Ontario Universal Influenza Immunization Program, interest and follow-through regarding influenza vaccination remain low among university students. Interest and follow-through were higher during the pandemic influenza season of 2009/10, but additional strategies are needed to improve interest and follow-through for vaccination.

\section{P099}

\section{SURVEILLANCE OF ADVERSE EVENTS FOLLOWING IMMUNIZATION (AEFI) WITH TRIVALENT INACTIVATED INFLUENZA VACCINES IN CANADA, 2000-2009}

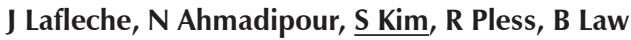 Ottawa, ON}

BACKGROUND: Vaccination campaigns for seasonal influenza are repeated every year with new vaccine formulations. Despite standard manufacturing processes, there have been variations in safety profile as exemplified by the signal involving "oculorespiratory syndrome" (ORS) during the 2000-2001 season. Careful surveillance of adverse events following immunization (AEFI) during each season remains a crucial component of each seasonal campaign.

OBJECTIVE: To summarize AEFI reported to the Public Health Agency of Canada (PHAC) for seasonal influenza vaccines over a ten year period in Canada.

METHODS: Data from October 2000 to September 2009 were extracted from the Canadian Adverse Event Following Immunization Surveillance System (CAEFISS) database for analysis. These cases are submitted to PHAC from local and provincial/territorial public health authorities. Cases were analysed by vaccine recipient demographics, vaccine, adverse events described, seriousness and other characteristics. A serious adverse event is one that results in hospitalization, death, permanent disability, or is life threatening.
RESULTS: From 2000-2009, there were more than 13,000 reports involving influenza vaccines out of nearly 45,500 total AEFI case reports received. The proportion of case reports involving TIV varies by season, but averages $20 \%$ of all reports received. However during the ORS season it rose to $50 \%$ and remained high for several subsequent years. The annual reporting rate ranged from 7 to 25 cases per 100,000 doses distributed, the latter figure during ORS. Two thirds of AEFI reports are in females and the mean age of vaccinees was 45 years. Allergic reactions and symptoms of ORS were the most commonly reported AEFIs, as were fever, fatigue, myalgia and rash. Less than $10 \%$ of reports are classified as serious.

CONCLUSION: AEFI reports for influenza vaccines comprise a significant number of reports received. Relatively few are serious. The ORS signal in 2000/2001 generated nearly 3 times the number of AEFI reports than what has been seen in other years. With the ORS signal as an exception, the AEFI profile has generally remained stable over time.

\section{P100 \\ INCREASE IN INFLUENZA VACCINATION RATES: THE IMPORTANCE OF DOCTOR 'BUY-IN'}

\section{NM Le Saux, R Grenon, N Barrowman, L Samson}

Ottawa, ON

BACKGROUND: In 2001, Ontario initiated publically funded influenza vaccine, regardless of risk category. In 2001, we determined a $27 \%$ vaccination rate for seasonal influenza in children presenting to the emergency department (ED) at the Children's Hospital of Eastern Ontario (CHEO). The objectives of this study were to estimate vaccination rates of seasonal influenza since 2001, of pH1N1, and to investigate attitudes toward influenza vaccination.

METHODS: A convenience sample of parents/guardians of children presenting to the ED and ambulatory clinics at the $\mathrm{CHEO}$ in March 2010 were surveyed. The questionnaire was administered by a research nurse. Vaccine uptake was compared to 2001.

RESULTS: 301 parents were surveyed. 280 (94.6\%) of the children had no underlying conditions. $113(37.5 \%)$ reported receiving seasonal flu vaccine at least once since 2001 and 23 (20.9\%) received it $>5$ times. 132 children $(43.9 \%)$ reported receiving $\mathrm{pH} 1 \mathrm{~N} 1$ vaccine. Immunization rates between children $\leq 2$ years and $>2$ years were not statistically different $(\mathrm{p}=.855) .111(36.9 \%)$ parents reported discussing the seasonal vaccine with their doctor. Of those who discussed pH1N1 vaccine with their doctor, $54.8 \%$ reported having their child vaccinated compared to $32.3 \%$ among those who did not $(\mathrm{p}<.001)$. Of those who reported receiving vaccine, the two most common reasons given were preventing the child from getting influenza $(31.2 \%)$ and preventing others in the household from illness (16.9\%). 83 (28.2\%) believed vaccine could cause 'flu', while 112 (39.9\%) believed vaccine had serious side effects, and 97 (33.4\%) believed pregnant women should receive influenza vaccine.

CONCLUSIONS: $43.9 \%$ of children had received $\mathrm{pH} 1 \mathrm{~N} 1$ vaccine, which was associated with discussion with their physician. At least $37 \%$ of children had received influenza vaccine at least once since provincial funding of vaccine. Targeted education of physicians and the public aimed at dispelling myths may further increase uptake of seasonal vaccine. 
P101

SENTINEL SURVEILLANCE FOR INFLUENZA IN CANADIAN HOSPITALS: EXPERIENCE OF THE PUBLIC HEALTH AGENCY OF CANADA (PHAC)/CANADIAN INSTITUTES OF HEALTH RESEARCH (CIHR) INFLUENZA RESEARCH NETWORK (PCIRN) ADULT SERIOUS OUTCOMES SURVEILLANCE NETWORK

SA McNeil ${ }^{1}$, Johnstone ${ }^{2}$, A Ambrose ${ }^{1}$, M Loeb ${ }^{2}$, M Russell ${ }^{3}$, S Trottier ${ }^{4}$, G Boivin ${ }^{4}$, A McCarthy ${ }^{5}$, E Henderson ${ }^{3}$, G Stiver ${ }^{6}$, S Halperin ${ }^{1}$, A McGeer ${ }^{7}$

${ }^{1}$ Halifax, NS; ${ }^{2}$ Hamilton, ON; ${ }^{3}$ Calgary, AB; ${ }^{4}$ Ste-Foy, QC; ${ }^{5} \mathrm{Ottawa}, \mathrm{ON} ;{ }^{6}$ Vancouver, $\mathrm{BC} ;{ }^{7}$ Toronto, ON

BACKGROUND: The PCIRN Adult Serious Outcomes Surveillance (SOS) Network was established to provide infrastructure for hospitalbased surveillance for serious outcomes (SO) due to influenza and for ongoing evaluation of vaccine effectiveness (VE).

METHODS: The PCIRN SOS Network comprises 8 hospitals and $\sim 5000$ acute care beds in 5 provinces. Nurses conducted active surveillance for influenza in admitted adults 10/1/09-4/30/10. All patients with respiratory infection, community acquired pneumonia (CAP), exacerbation of chronic obstructive pulmonary disease (COPD)/asthma, sepsis, or febrile cardiac disease had NP swab for influenza PCR. Testnegative patients were controls for calculation of VE.

RESULTS: 273 cases of lab-confirmed pH1N1 were enrolled; 51\% female, mean age $47 y$ (16-87y). In 41\% admitting diagnoses included influenza or influenza-like illness (ILI); other admitting diagnoses included CAP (37\%), exacerbation of chronic disease (17\%), and sepsis (3\%). Only $34 \%$ and $38 \%$ of cases met the PHAC or CDC ILI case definitions, respectively. Overall $83 \%$ had at least 1 comorbidity, $6 \%$ were pregnant, $8.5 \%$ were morbidly obese (BMI over 40 ) and $2.5 \%$ were Aboriginal. 33 patients received $\mathrm{pH} 1 \mathrm{~N} 1$ vaccine; only 2 were vaccinated $>14 \mathrm{~d}$ prior to onset of illness. Overall, $38 \%$ had at least 1 complication; CAP was most common (102/128; $80 \%$ patients with available data); $8 \%$ experienced exacerbation of chronic disease; $29 \%$ were admitted to ICU and $22 \%$ were ventilated. Mean length of stay was $11 \mathrm{~d}(1-123 \mathrm{~d}) .17$ (6\%) died; patients who died were older $(55 \mathrm{y}$ vs 46y, $\mathrm{p}=.02$ ).

CONCLUSIONS: The PCIRN SOS Network provides critical infrastructure to characterize clinical features and outcomes of influenza in hospitalized adults. Even during a pandemic, $<50 \%$ of cases had an admitting diagnosis of influenza and 2/3 of cases would have been missed if presence of ILI was used to guide influenza testing highlighting the importance of active surveillance to better define influenza burden of disease. Ability to estimate VE this season was limited because the pandemic peak occurred before widespread access to $\mathrm{pH} 1 \mathrm{~N} 1$ vaccine.

\section{P102 \\ IMPACT OF HOSPITALIZATION OF PREGNANT WOMEN FOR INFLUENZA LIKE ILLNESS (ILI) ON INFANT OUTCOMES}

\footnotetext{
SA McNeil ${ }^{1}$, L Dodds ${ }^{1}$, D Fell ${ }^{1}$, M Steinhoff $^{2}$, N MacDonald ${ }^{1}$

${ }^{1}$ Halifax, NS; ${ }^{2}$ Cincinnati, OH

BACKGROUND: Analysis of neonatal outcome data from the Bangladesh RCT of influenza vs pneumococcal vaccine during pregnancy demonstrated an increased proportion of small for gestational age (SGA) infants and lower mean birth weights among babies born to mothers not protected by influenza vaccine. This suggests that prevention of influenza in pregnancy results in improved fetal growth (Steinhoff et al, PAS, May 2010). Evidence for an influenza effect on fetal outcome in high resource regions is lacking. We compared outcomes of infants born to women with and without hospitalization for ILI over a 10 year period in Nova Scotia (NS) Canada using previously described data that showed increased risk of ILI hospitalization in influenza season (CMAJ 2007; 176: 463-468).
}

METHODS: Using the NS Perinatal database, neonatal outcomes among singletons were compared between mothers with or without admission for ILI during pregnancy. Multivariate logistic regression was used to examine infant outcomes, adjusting for maternal high risk status and other potential confounders.

RESULTS: Neonatal outcomes in mothers with or without admission for ILI during pregnancy:

\begin{tabular}{|c|c|c|c|c|}
\hline \multirow{2}{*}{$\begin{array}{l}\text { Outcome } \\
\text { amongst } \\
\text { singletons } \\
\text { ( } n=132,588)\end{array}$} & \multicolumn{2}{|c|}{$\begin{array}{l}\text { Any hospitalization for resp } \\
\text { illness during preg }\end{array}$} & \multirow[b]{2}{*}{$\begin{array}{l}\text { Unadjusted RR } \\
(95 \% \mathrm{Cl})\end{array}$} & \multirow[b]{2}{*}{$\begin{array}{l}\text { Adjusted RR } \\
(95 \% \mathrm{Cl})\end{array}$} \\
\hline & $\begin{array}{l}\text { Yes }(n=489) \\
n(\%)\end{array}$ & $\begin{array}{c}\text { No }(n=132,099) \\
n(\%)\end{array}$ & & \\
\hline $\begin{array}{l}\text { Peri/post-natal } \\
\text { death }\end{array}$ & $4(.8)$ & $996(.7)$ & $1.09,0.41-2.92$ & $0.94,0.34-2.64$ \\
\hline $\begin{array}{l}\text { Low birth weight } \\
\quad(<2500 \mathrm{~g})\end{array}$ & $28(5.7)$ & $6035(4.6)$ & $1.27,0.87-1.86$ & $1.05,0.62-1.77$ \\
\hline $\begin{array}{l}\text { Preterm } \\
\text { (<37 weeks) }\end{array}$ & $31(6.3)$ & $7159(5.4)$ & $1.18,0.82-1.70$ & $0.98,0.68-1.41$ \\
\hline Neonatal morbidity & $44(9)$ & $6488(4.9)$ & $1.92,1.40-2.61$ & $1.78,1.29-2.46$ \\
\hline Mean birth weight & 3374.6 (SD 594) & 3468.3 (SD 584) & $\begin{array}{l}B=-93.71 \\
(p=.0004)\end{array}$ & $\begin{array}{c}B=-60.14 \\
(p=.006)\end{array}$ \\
\hline $\begin{array}{l}\text { Small for } \\
\text { gestational age }\end{array}$ & $62(13)$ & $12594(9.7)$ & $1.39,1.06-1.82$ & $1.35,1.03-1.76$ \\
\hline
\end{tabular}

IMPLICATIONS: These analyses extend findings of the Bangladesh RCT SGA findings to apply to infants born to women in a high resource country. While supporting a negative impact of influenza on neonatal birthweight, our findings are indirect as ILI was not confirmed as influenza. More research is needed to determine whether influenza vaccine during pregnancy in high resource regions can decrease rates of ILI admissions among pregnant women and their infants and decrease rates of SGA babies.

\section{P103}

ASSESSING CANADIAN HEALTH CARE PROVIDERS
PREFERENCE FOR ENHANCED INFLUENZA VACCINE
CHARACTERISTICS AND FOR ENHANCED INFLUENZA
VACCINATION CONVENIENCE FACTORS QUANTIFIED
USING OUT OF POCKET WILLINGNESS TO PAY

A Chuck ${ }^{1}$, P Jacobs ${ }^{1}$, PI Murray ${ }^{2}$

${ }^{1}$ Edmonton, $\mathrm{AB} ;{ }^{2}$ Toronto, $\mathrm{ON}$

BACKGROUND: Influenza vaccination of healthcare providers (HCP) with direct patient contact is strongly recommended in Canada. Yet, HCP coverage rates remain well below National targets. A better understanding of HCP preferences through "willingness-topay" (WTP) economic studies may help guide initiatives to increase vaccine uptake.

OBJECTIVES: This study assessed HCP preferences for influenza vaccination in terms of vaccine and convenience characteristics, quantified by out-of-pocket WTP costs.

METHODS: The study was an observational design using a web-based discrete-choice survey. Thirty scenarios were presented to participants, differing in vaccine administration characteristics, convenience factors and out-of pocket cost expectation. Enhanced vaccine characteristics included the value of shorter needles and thimerosal free vaccines. Enhanced convenience factors included the value of earlier availability date, decreased waiting time and no appointment. Analysis was stratified based on participants' intention to be vaccinated and further, by offering all enhancements at no cost.

RESULTS: $71 \%(n=40 / 56)$ of HCPs intended to be vaccinated at the start of the survey, and were willing to pay $\$ 12.63$ for enhanced vaccine characteristics and $\$ 19.09$ for enhanced convenience factors. The WTP costs were statistically significantly higher $(\mathrm{p}<0.05)$ than the non-enhanced scenario. Offering the enhanced scenarios at no cost resulted in $84 \%(n=47 / 56)$ intending to be vaccinated (an additional $13 \%(n=7 / 56)$ of HCP intending to be vaccinated). Overall, HCP not 
intending to be vaccinated significantly valued the vaccine characteristics (\$24.76) compared with the non-enhanced scenario; no significant value was reported for enhanced convenience.

CONCLUSION: Enhanced vaccine characteristics and enhanced convenience factors resulted in an increased WTP for influenza vaccination. Addressing both factors also increased HCP intention to be vaccinated when costs were removed, and therefore, may be utilized to increased vaccine uptake among HCP.

\section{P104 \\ INFLUENZA VACCINATION AND DAYCARE WORKERS: COMPATIBLE OR NOT?}

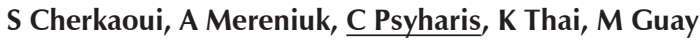

Longueuil, QC

CONTEXT: Although vaccine coverage estimates are made nationwide for seasonal and $A(H 1 N 1) p$ influenza for health care workers, the situation pertaining to daycare centre workers (DCW) remains unknown.

PURPOSE: This study aims to evaluate the acceptability of the seasonal and $\mathrm{A}(\mathrm{H} 1 \mathrm{~N} 1) \mathrm{p}$ influenza vaccines among DCW in the Montérégie area, Quebec. It also looks into the perceptions and the factors influencing DCW's decision to be vaccinated.

METHODS: A descriptive cross-sectional study was conducted in January 2010 using a self-administered questionnaire (based on the Health Belief Model) available in an electronic or paper version. The questionnaire was distributed to DCW at 12 daycare centres and to 40 administrative members of the Regroupement des Centres de la Petite Enfance de la Montérégie.

RESULTS: The response rate was 57\% (194/339). Vaccination coverage amongst DCW totals $26 \%$ for seasonal influenza (estimated over a three-year period) compared to $61 \%$ for $\mathrm{A}(\mathrm{H} 1 \mathrm{~N} 1) \mathrm{p}$ influenza. The position held within the establishment (administrator vs. caregiver), a greater number of years working in childcare ( $>10$ vs. $\leq 9$ years), and the history of a yearly medical follow-up were determining factors in the decision to get vaccinated $(\mathrm{p}<0.03)$. Health professionals remain the main source of information on immunization for $85 \%$ of DCW. The reason motivating $88 \%$ of DCW to receive the vaccine is the will to protect others. DCW who refuse vaccination base their decision on the lack of confidence in the newly developed A(H1N1)p vaccine $(57 \%)$, and on the perception of non-vulnerability concerning seasonal influenza (53\%).

CONCLUSION: In order to improve vaccination coverage among $\mathrm{DCW}$, further education on the impacts of the $\mathrm{A}(\mathrm{H} 1 \mathrm{~N} 1) \mathrm{p}$ and the seasonal influenzas and their corresponding vaccines is recommended.

\section{P105}

\section{INFLUENZA VACCINATION RATES DECREASE WITH} INCREASING AGE AMONG CHILDREN < 9 YEARS OF AGE IN CALGARY, ALBERTA

EA Medd, ML Russell, EA Henderson, M Kirkwood, J MacDonald Calgary, AB

BACKGROUND: There are few reports of influenza vaccination coverage among young children in Canada. A primary series for influenza vaccination in children consists of 2 doses of vaccine administered after the age of 6 months. If given in the same season, the doses must be separated by at least 28 days. Subsequently, children require only one annual dose of vaccine. Estimations of influenza vaccination coverage among children have not usually taken this into account.

PURPOSE: Over 2004-2008, to estimate age-specific influenza vaccination coverage among children $0.5-8$ years of age in Calgary, Alberta.

METHODS: Numerator data with unique identifiers were extracted from the Alberta Health Services immunization information system, 2004-2008. In each year, children were considered to be vaccinated if they completed a primary series, or if having already received a primary series, received a single dose of the vaccine of the year. Age-specific population counts were taken from the Alberta Health Care Insurance Program Registry, which is updated annually.

RESULTS: Among children 0.5-8 years of age, annual influenza vaccination coverage increased from 7 to $14 \%$ from 2004 to 2008 . Coverage rates decreased with increasing age in all years.

TABLE 1

Age-specific influenza vaccination coverage among children in Calgary, Alberta, 2004-2008

\begin{tabular}{ccccc}
\hline Age (years) & $\mathbf{2 0 0 4 - 0 5}$ & $\mathbf{2 0 0 5 - 0 6}$ & $\mathbf{2 0 0 6 - 0 7}$ & $\mathbf{2 0 0 7 - 0 8}$ \\
\hline $0.5-<1$ & 26 & 44 & 38 & 36 \\
$1-<2$ & 19 & 31 & 30 & 32 \\
$2-<3$ & 6 & 10 & 13 & 12 \\
$3-<4$ & 5 & 8 & 12 & 11 \\
$4-<5$ & 4 & 7 & 11 & 12 \\
$5-<6$ & 3 & 5 & 10 & 9 \\
$6-<7$ & 3 & 4 & 9 & 8 \\
$7-<8$ & 2 & 3 & 8 & 7 \\
$8-<9$ & 2 & 3 & 7 & 6 \\
All Ages & 7 & 11 & 14 & 14 \\
\hline
\end{tabular}

CONCLUSIONS: Influenza vaccination coverage is low among young children, especially among those $2-8$ years of age. Strategies for improving coverage include expanding eligibility criteria for publicly funded vaccines and adding school based vaccine delivery.

\section{P106 \\ SEROLOGICAL RESPONSE TO REPEATED INFLUENZA IMMUNIZATION AMONG CHILDREN}

S Rafiq ${ }^{1}$, K Fonseca ${ }^{2}$, P Singh ${ }^{1}$, M Smieja ${ }^{1}$, ML Russell ${ }^{2}$, M Loeb ${ }^{1}$

${ }^{1}$ Hamilton, ON; ${ }^{2}$ Calgary, AB

BACKGROUND: The National Advisory Committee on Immunization (NACI) indicates that annual vaccination against seasonal influenza is recommended for children aged 6 months or older. The impact of repeated vaccination on serological response among children is not clear and at least one study in adults has shown lower hemagglutination inhibition assay (HAI) titres post immunization in those who had been previously vaccinated with trivalent inactivated influenza vaccination (TIV). We wondered if children who were repeatedly immunized (i.e., more than one season) with TIV would show reduced immunogenicity.

PURPOSE: We describe the mean fold GMT antibody responses by number of seasons in which the children were vaccinated.

METHODS: Participants were healthy children enrolled at age 3-15 years in a 3-year study of influenza transmission conducted on Hutterite colonies in central Alberta. They were vaccinated and had serology done before and again 4 weeks after vaccination in 2007-2008. Participants had been offered age appropriate vaccination (NACI guidelines) with seasonal TIV in 2005-06, 2006-07 and 2007-08. Pre and post vaccination fold changes in geometric mean titres (GMT) of the HAI response to $\mathrm{A} / \mathrm{H} 1 \mathrm{~N} 1$ Solomon Islands, $\mathrm{A} / \mathrm{H} 3 \mathrm{~N} 2 \mathrm{~W}$ isconsin, $\mathrm{B} /$ Florida were compared by number of seasons in which children had been vaccinated $(3,2$ or 1$)$.

RESULTS: Children who were immunized more frequently did not show consistently reduced changes in GMT than those who had received fewer annual vaccinations. For the H1N1 antigen, the mean fold increase in GMT by number of seasons vaccinated was 22.0 vs. 6.1 vs. 19.7 (for 3, 2 or 1 seasons). For $\mathrm{H} 3 \mathrm{~N} 2$, the fold increases were 8.4 vs. 2.2 vs. 25.1 respectively. For influenza B, the fold increases were 4.1 vs. 2.4 vs. 6.4

CONCLUSION: There does not appear to be a ceiling effect with repeated TIV vaccination based upon these data. 
P107

AN ECONOMIC APPRAISAL OF ONTARIO'S UNIVERSAL INFLUENZA IMMUNIZATION PROGRAM: A COST-UTILITY ANALYSIS

B Sander $^{1}$; JC Kwong1; CT Bauch ${ }^{2}$; A Maetzel ${ }^{3}$; A McGeer ${ }^{1}$;

J Raboud ${ }^{1}$; MD Krahn ${ }^{1}$

${ }^{1}$ Toronto, ON; ${ }^{2}$ Guelph, ON; ${ }^{3}$ Basel, Switzerland

BACKGROUND: In July 2000, the province of Ontario, Canada, initiated a universal influenza immunization program (UIIP) to provide free seasonal influenza vaccines for the entire population. This is the first large-scale program of its kind worldwide. The objective of this study was to conduct an economic appraisal of Ontario's UIIP compared to a targeted influenza immunization program (TIIP).

METHODS: A cost-utility analysis using Ontario health administrative data was performed. The study was informed by a companion ecological study comparing physician visits, emergency department visits, hospitalizations, and deaths between 1997 and 2004 in Ontario and nine other Canadian provinces offering targeted immunization programs. The relative change estimates from pre-2000 to post-2000 as observed in other provinces were applied to pre-UIIP Ontario event rates to calculate the expected number of events had Ontario continued to offer targeted immunization. Main outcome measures were quality-adjusted life years (QALYs), costs in 2006 Canadian dollars, and incremental cost-utility ratios (incremental cost per QALY gained). Program and other costs were drawn from Ontario sources. Utility weights were obtained from the literature. The incremental cost of the program per QALY gained was calculated from the health care payer perspective.

RESULTS: Ontario's UIIP costs approximately twice as much as a targeted program but reduces influenza cases by $61 \%$ and mortality by $28 \%$, saving an estimated 1,134 QALYs per season overall. Reducing influenza cases decreases health care services cost by $52 \%$. Most cost savings can be attributed to hospitalizations avoided. The incremental cost-effectiveness ratio is Can $\$ 10,797 / \mathrm{QALY}$ gained. Results are most sensitive to immunization cost and number of deaths averted.

CONCLUSIONS: Universal immunization against seasonal influenza was estimated to be an economically attractive intervention.

\section{P108 \\ PRECLINICAL AND CLINICAL DEVELOPMENT OF PLANT-BASED VIRUS-LIKE PARTICLE VACCINE FOR INFLUENZA H5N1 STRAINS}

\section{$\underline{S \text { Trepanier, N Landry, L Vezina }}$}

Quebec, QC

BACKGROUND: In the event of an influenza pandemic, current manufacturing technology is not suitable to provide large number of vaccine doses quickly. Medicago developed an innovative technology for producing virus-like particle (VLP) influenza vaccines using non-transgenic plants that has a minimum lag-time and allows vaccine production within three weeks of the identification of the genetic sequence from a pandemic strain.

PURPOSE: First, the efficacy of the vaccine to protect against a lethal challenge with a heterologous $\mathrm{H} 5 \mathrm{~N} 1$ strain was assessed in ferrets. The purpose of the Phase I clinical study was to assess the safety and immunogenicity of Medicago's plant-based VLP influenza vaccine, developed against $\mathrm{H} 5 \mathrm{~N} 1$ strains.

METHODS: Ferrets received two doses of 1.8 or $3.7 \mu \mathrm{g}$ alum-adjuvanted VLP vaccine 21 days apart or alum alone. Ferrets were challenged with a heterologous $\mathrm{H} 5 \mathrm{~N} 1$ virus 45 days after the second dose. In a Phase I, randomized, double-blind study, groups of 12 healthy adults received two doses of 5, 10 or $20 \mu \mathrm{g}$ of alum-adjuvanted VLP vaccine or placebo 21 days apart. Solicited local and systemic reactions were recorded for 7 days after each vaccination and immunogenicity of the vaccine was assessed. Antibodies to plant glycans were also investigated in sera of vaccinated humans.
RESULTS: The vaccine provided $100 \%$ protection against lethal challenge with heterologous H5N1 influenza strain in ferrets. In humans the VLP vaccine against $\mathrm{H} 5 \mathrm{~N} 1$ strains was well tolerated and induced a robust immune response. The vaccine did not induce detectable levels of antibodies specific to plant gylcans.

CONCLUSION: Data obtained from preclinical and clinical studies combined with Medicago's simple and rapid technology make such VLP influenza vaccines a very attractive product to include in the seasonal and pandemic plans of Canada and other countries.

\section{P109}

\section{MEDICAL CLASSIFICATION OF CASE REPORTS OF ADVERSE EVENTS FOLLOWING IMMUNIZATION (AEFI) WITH PANDEMIC H1N1 VACCINES}

\section{H Anyoti, J Lafleche, N Ahmadipour, R Pless, B Law Ottawa, ON}

BACKGROUND: MedDRA (Medical Dictionary for Regulatory Activities) is a thesaurus used worldwide to code and classify adverse event (AE) information from case reports. This facilitates analysis of AEFIs by enabling standardization despite differences in case documentation by reporters. Over 65,000 "Lower Level Terms" (LLT) are available, mapping up to 18,000 preferred terms (PTs) and 26 Primary System Organ Classes (pSOCs), allowing for grouping of AEFIs and safety analysis.

PURPOSE: To present an overview of the processing of AEFIs received federally, including the principles of coding into MedDRA and an example of analysis using reports received for pandemic H1N1 vaccines during the recent pandemic influenza campaign.

METHODS: Case reports received are entered into the Canadian Adverse Event Following Immunization Surveillance System database. Medical reviewers examined each AEFI listed or described, assigning the appropriate LLT. PT and pSOC fields are auto populated, and reports analyzed based on PT and pSOC.

RESULTS: A total of 1,362 unique LLTs were applied to the 2,947 reports received by March 13th, 2010. These mapped to 578 unique PTs and 23 pSOCS. The average number of terms per report were 4, 3.8 and 2.6 for LLT, PT and pSOC respectively. The most common pSOCs were General disorders and administration site conditions (50\% of reports), Nervous system disorders (38\%), Skin and subcutaneous tissue disorders (37\%), and Gastrointestinal disorders (29\%). The hierarchy permitted a rapid review of age group and gender differences for AEFI, as well as enabled focus on specific types of events for more detailed assessment.

CONCLUSION: The large number of LLTs allow retention of originally reported information, facilitating analysis of AEFI at a broader level. This becomes useful to describe overall trends and apply standardized data queries looking for signals of concern. In addition, the global use of MedDRA allows data to be compared internationally, but training is crucial to ensure standardization of coding practice.

\section{P110}

DELIVERY OF AN OCCUPATIONAL HEALTH (OH) PANDEMIC INFLUENZA VACCINE 2009 INFLUENZA A (H1N1) PROGRAM: DESCRIPTION OF A PARTNERSHIP BETWEEN A CLINICAL RESEARCH FACILITY AND A HOSPITAL OH PROGRAM

DM Baxendale, WC Allen, JM Langley, SA Halperin, CL Lockhart, LM Dixon, KS Green, SG Ashton, CJ Walls, DM Boliver

Halifax, NS

INTRODUCTION: Immunization of health care workers (HCW) has high priority during pandemics in order to maintain continuity of healthcare. We describe an innovative partnership between the CCfV clinical research facility and the IWK $(\mathrm{OH}$, Chief Nursing Office, 
Quality \& Patient Safety, Pharmacy), a university-affiliated health care centre.

METHODS: The NS Dept. of Health Promotion and Protection (HPP) provided vaccine; human and material resources were the responsibility of each facility. The CCfV was contracted by $\mathrm{OH}$ to deliver the pandemic and seasonal influenza vaccines. Four research clinic rooms were used and a meeting room converted to 3 patient stations. Clinic times were publicized internally (e.g. intranet, posters, through managers). Vaccination was voluntary and required written consent. Roving clinics were offered to deliver vaccine to staff in patient care areas by IWK nurses.

RESULTS: From 26/9/09 to 19/11/09, 3401 persons were immunized; $47 \%$ of these in the first month. Of the total, 2555 (75\%) were immunized at CCfV, 784 (23\%) via roving clinics and 62 immunized outside the IWK. Vaccine coverage was $74 \%$ of employees and $92 \%$ of non-employees in direct patient care. Daily $6 \mathrm{am}-8 \mathrm{pm}$ clinics required a coordinator, registration staff and vaccinators. Changes in sequencing prioritization by HPP because of vaccine supply, so that only those with direct patient care could be immunized, necessitated crowd control and verification by interview of status as a HCW with patient contact. Conduct of studies underway was not impeded, due to CCfV staff's willingness to work flexible hours. $13 \mathrm{CCfV}$ nurses and 7 research assistants devoted 700 person-hours to run the CCfV clinic and 70 IWK nurses devoted 215 person-hours to the roving clinics (both 3.6 immunizations/man-hour).

CONCLUSIONS: An academic clinical research center provided human resources and a clinic area for mass immunization of $\mathrm{HCW}$ s during an influenza pandemic. $\mathrm{OH}$ programs without internal surge capacity to deliver large amounts of vaccine in a short period may benefit from this model of collaboration with clinical researchers.

\section{P111}

SAFETY OF THE 2010-2011 INFLUENZA VACCINE IN HEALTH CARE WORKERS (HCW) PREVIOUSLY VACCINATED IN 2009 WITH ADJUVANTED PH1N1 VACCINE M Benoit ${ }^{1}$, M Gariépy ${ }^{1}$, A Kallos ${ }^{2}$, B Coleman ${ }^{3}$, A Ambrose ${ }^{4}$, I Rouleau ${ }^{1}$, S McNeil ${ }^{5}$, A McGeer ${ }^{4}$, J Bettinger ${ }^{3}$, G De Serres ${ }^{1,6}$ ${ }^{1}$ Quebec, QC; ${ }^{2}$ Vancouver, BC; ${ }^{3}$ Toronto, ON; ${ }^{4}$ Halifax, NS BACKGROUND: In 2009, the adjuvanted monovalent pH1N1 influenza vaccine frequently caused local reactions and fever. Greater reactogenicity with subsequent revaccination with the non-adjuvanted trivalent inactivated seasonal influenza vaccine (TIV) is unlikely but this remains to be established. We conducted the active surveillance of $>10000 \mathrm{HCW}$ vaccinated in 2009 with the adjuvanted monovalent H1N1 vaccine who will receive the 2010-2011 TIV.

METHODS: In 2009, a network of hospitals conducted active electronic surveillance of $>5000 \mathrm{HCW}$ s immunized against $\mathrm{pH} 1 \mathrm{~N} 1$. For 2010-2011, the network will be expanded to follow-up $10000 \mathrm{HCW}$. The HCWs of four to six hospitals from various provinces will be invited to participate in a web-based active surveillance of vaccine safety. The main outcome $(\mathrm{MO})$ will be the onset after vaccination of any new health problem or the exacerbation of an existing condition severe enough to cause work absenteeism or require medical consultation. Participants will be contacted 7, 14 and 28 days after vaccination by email and provided with a hyperlink to a secure website for a brief survey. Participants reporting the main outcome will be contacted by a nurse for detailed information.

RESULTS: At the time of CIC, we will have completed the 7 day followup. We will present the participation rate, the frequency of HCW who will have suffered from the main outcome during the 1st week after vaccination with an emphasis on local reaction and fever sufficient to cause absenteeism or medical consultation. We will compare this to the results of the 2009 adjuvanted monovalent vaccine.

CONCLUSIONS: The PCIRN vaccine safety network has been established to provide rapid information on the safety of the influenza vaccine. Results presented within a month after the onset of the
2010-2011 vaccination campaign should demonstrate the feasibility of timely safety information on a large group of vaccinees.

\section{P112}

PARENTAL KNOWLEDGE, ATTITUDES AND BELIEFS AROUND PANDEMIC H1N1 IMMUNIZATION IN BRITISH COLUMBIA

JA Bettinger ${ }^{1}, M$ Dawar ${ }^{1}$, P Hasselback $^{2}, M_{\text {Naus }}{ }^{1}$

${ }^{1}$ Vancouver, BC; ${ }^{2}$ Kelowna, BC

BACKGROUND: In April 2009 a new pandemic strain of influenza emerged ( $\mathrm{pH} 1 \mathrm{~N} 1$ ). Immunization for $\mathrm{pH} 1 \mathrm{~N} 1$ was expected to occur in Canada in late fall. Given the US experience with pandemic vaccine in 1976 and increasing concerns by parents and media on vaccine safety, an understanding of parental knowledge, attitudes and beliefs (KAB) around pH1N1 immunization was important.

PURPOSE: To inform immunization planners of parental KAB that could influence $\mathrm{pH} 1 \mathrm{~N} 1$ vaccine acceptance and uptake.

METHODS: From August 26 - October 5, 2009 eight focus group discussions were conducted with parents of children $<10$ years of age in urban and rural areas of British Columbia. A coding scheme to identify major themes was generated in an iterative manner based on grounded theory methodology.

RESULTS: A total of 51 parents participated; $86 \%$ were female, the mean age was 31 years (range 20-45) and 39\% had received prior seasonal vaccine. Parents were not well-informed about pH1N1. Misconceptions about $\mathrm{pH} 1 \mathrm{N1}$ and seasonal influenza vaccines were common across groups. Parents recognized their lack of knowledge and wanted more information about their risk, their children's risk and the effectiveness and safety of the pH1N1 vaccine before they would immunize their children.

The trustworthiness of information sources was an important theme. Participants wanted honest, factual information from "trusted sources", such as health care providers, select websites, friends and family.

Most parents expressed concerns about $\mathrm{pH} 1 \mathrm{~N} 1$ vaccine safety, particularly the speed in which the vaccine had been developed, the lack of clinical trial data in children and the lack of long-term follow up data.

CONCLUSION: The realities of pandemic and seasonal influenza vaccine production result in limited availability of clinical data at the time of a program launch; therefore some concerns identified in our focus groups may not be readily addressed for those who are highly vaccine risk averse. Results from our focus groups can be used to design better public communication about the scientific decision-making process related to seasonal influenza vaccines. This may provide better understanding during pandemic conditions.

\section{P113}

\section{LIFE OF IMPACT (CANADIAN IMMUNIZATION MONITORING PROGRAM, ACTIVE) NURSE MONITORS DURING THE PANDEMIC INFLUENZA A H1N1}

\section{Piché, $\underline{S}$ Bouchard \\ Montreal, QC}

BACKGROUND: Pandemic influenza A H1N1 (pH1N1) increased the workload for IMPACT nurse monitors across Canada. Capturing the large number of cases and increased vigilance for adverse events following a mass immunization campaign was challenging. IMPACT is a paediatric hospital-based active surveillance program for adverse events following immunization (AEFI) and selected vaccine preventable diseases since 1991.

OBJECTIVE: Describe how the IMPACT monitors collected data on a large number of influenza cases and review how they conducted surveillance for AEFI's during the H1N1 pandemic. We will describe a few case reports of paediatric influenza admissions and AEFI post $\mathrm{pH} 1 \mathrm{~N} 1$ vaccination. 
METHODS: The nurse monitors have been conducting surveillance for paediatric Influenza admissions since the 2003-04 seasons. Reporting is done in two steps. The initial report is an electronic weekly submission of the number of cases, age group and deaths per site. The second is a detailed case report. Due to the large number of cases, the reporting system was adjusted for the second wave to accommodate rapid reporting and focus attention on cases needing intensive care, prolonged hospitalization and deaths. The period of surveillance for neurological events post $\mathrm{pH} 1 \mathrm{~N} 1$ vaccine was extended.

RESULTS: IMPACT reported a mean of 423 influenza cases per year for 2003-2009 with a maximum of 505 cases. The monitors reported 324 cases for the first wave (to August 31st, 2009). There were 961 reports for the second wave including 264 cases in 1 week at the peak. Impact monitors' workload increased in order to meet the demands in a timely manner.

CONCLUSION: The pandemic first and second wave and mass vaccination campaign were busy times for everyone. Despite this, the part-time nurse monitors were able to provide, in real-time, data to the Public Health Agency of Canada on the impact of the Pandemic H1N1 disease in Canadian children.

\section{P114 \\ PANDEMIC H1N1 VACCINE COVERAGE IN TORONTO USING COMMUNITY-BASED HEALTH CARE PROVIDERS AND PUBLIC HEALTH CLINICS, 2009-2010}

F Kolbe, S Ota, E Gournis, J Cameron, B Yaffe

Toronto, ON

BACKGROUND: Vaccine for pandemic H1N1 (pH1N1) was administered by community-based health care providers (e.g. physicians, hospitals, long-term care homes) and by public health at immunization clinics in Toronto.

OBJECTIVE: To describe and compare individuals receiving $\mathrm{pH} 1 \mathrm{~N} 1$ vaccine from community-based health care providers to those vaccinated at public health clinics to inform future planning for large scale vaccination efforts.

METHODS: Public health clinics recorded individual-level vaccination data for all recipients of the vaccine. These data included: vaccination date, age, gender, and priority group for vaccination as prescribed by provincial health authorities. Community-based health care providers collected and submitted to Toronto Public Health individuallevel non-identifiable immunization data for the first four weeks of the campaign, after which aggregate weekly totals were submitted.

RESULTS: An estimated 748,510 individuals (28.2\% of the population) were vaccinated in Toronto. The majority of vaccinations ( $n=516,835$ or $69 \%$ ) were administered by the 1,208 community-based health care providers who requested vaccine. Public health vaccinated 231,675 individuals attending one of 390 immunization clinics offered at 12 locations across Toronto, or at one of 119 homeless/under-housed shelters and drop-in centres. Overall, more females received vaccinations than males $(54.9 \%)$ which did not differ between those vaccinated by community-based health care providers or at public health clinics. The median age of those vaccinated was 39 years. Age-specific vaccination rates were highest in the $1-4$ year age group $(35,665$ per $100,000)$ and lowest in the $15-19$ year age group $(8,228$ per 100,000$)$. The age distribution did not differ between those vaccinated by community-based providers or at public health clinics.

CONCLUSION: The majority of $\mathrm{pH} 1 \mathrm{~N} 1$ vaccine was administered by community-based health care providers. The age and gender distribution did not differ significantly between those vaccinated by community-based health care providers or at public health clinics. Future vaccination campaigns should focus on collaboration with community providers.
P115

IMMUNOGENICITY OF A REDUCED DOSE OF
AREPANRIX $^{\mathrm{TM}}$ H1N1 IN HEALTHY ADULTS

BL Coleman ${ }^{1}$, J Gubbay ${ }^{1}$, DW Scheifele ${ }^{2}$, Y Li $^{3}$, D Low ${ }^{1}$,

T Mazzulli $^{1}$, L Shi ${ }^{1}$, K Green ${ }^{1}$, S Halperin ${ }^{4}$, B Law ${ }^{5}$, A McGeer ${ }^{1}$

${ }^{1}$ Toronto, ON; ${ }^{2}$ Vancouver, BC; ${ }^{3}$ Winnipeg, MB; ${ }^{4} \mathrm{Halifax}, \mathrm{NS}$;

${ }^{5}$ Ottawa, ON

PURPOSE: This study was undertaken to determine if a $1 / 2$ dose of Arepanrix would provide adequate immunogenicity, allowing consideration of dose sparing in the event of vaccine shortage.

METHODS: Healthy 18 to 60 year olds were enrolled from 29/10/09 to $13 / 01 / 10$. Blood was taken before vaccination with $1 / 2$ dose Arepanrix (1.9 $\mu \mathrm{g} \mathrm{HA})$ and at days 14 and/or 21, and day 90. Adults (20 to 59 years old) enrolled in a concurrent study of immunogenicity following a full dose of Arepanrix between 9/11/09 and 25/11/09 are used as a comparison.

RESULTS: 98 adults received $1 / 2$ dose vaccine: 63 (64\%) were females; median age 47 years. 50 adults received full dose vaccine: 19 (38\%) were females; median age 51 years. Results were not significantly different between the groups with $34 / 98$ (35\%) $1 / 2$ dose and $11 / 50$ (22\%) full dose recipients seroprotected $(\mathrm{HA} \geq 1: 40)$ at baseline $(\mathrm{P}=0.11)$ and $78 \%$ of $1 / 2$ and $76 \%$ of full dose subjects seroprotected at day $21 \quad(P=0.76)$. Participants 46-60 years of age were less likely to be seroprotected at day 21 than those $20-45$ years old in both the $1 / 2$ dose $(P=0.04)$ and full dose $(P=0.02)$ groups. Response in the $1 / 2$ and full dose groups did not differ significantly in geometric mean titre (GMT) at baseline (19 vs. 16) or day 21 (124 vs. 109) or in seroconversion ratio (6.5 vs. 7.0). Similarly, the proportion of participants that seroconverted was $63 \%$ and $70 \%$, in the $1 / 2$ and full dose groups, respectively $(\mathrm{P}=0.42)$. There was no difference for $1 / 2$ dose participants' GMT $(n=73)$ at day 21 and day 90 (234 vs. 205).

CONCLUSION: Immunogenicity of a reduced dose of this AS-03 adjuvanted $\mathrm{pH} 1 \mathrm{~N} 1$ influenza vaccine was similar to full dose responses in adults $18-60$ years old. This is a possible strategy for expanding the availability in the event vaccine shortage.

\section{P116 \\ PCIRN RANDOMIZED TRIAL TO COMPARE THE IMMUNOGENICITY OF ADJUVANTED PANDEMIC H1N1 2009 VACCINE WITH OR WITHOUT A SECOND "BOOSTER" DOSE IN HIV INFECTED ADULTS C Cooper $^{1,2}$, D Haase ${ }^{2}$, S Walmsley ${ }^{2,3}$, M Klein ${ }^{2,4}$, DW Scheifele ${ }^{5}$, S Halperin2, Y Li'2,6, B Law ${ }^{1,2}$, B Smith ${ }^{2}$ ${ }^{1}$ Ottawa, ON; ${ }^{2}$ Halifax , NS; ${ }^{3}$ Toronto, ON; ${ }^{4}$ Montreal, QC; ${ }^{5}$ Vancouver, BC; ${ }^{6}$ Winnipeg , MB}

BACKGROUND: Poor influenza vaccine responses and effectiveness and more severe influenza disease in HIV-infected persons necessitate improved immunization strategies.

OBJECTIVE: To determine if giving HIV-infected adults a second "booster" dose of adjuvanted pandemic influenza vaccine would improve upon single-dose protection.

METHODS: A randomized, controlled trial was conducted at 4 PCIRN centers. HIV-infected adults (20-59 years old) were randomly assigned to receive one (Group A) or two doses (Group B) of monovalent, adjuvanted H1N1 2009 vaccine (Arepanrix ${ }^{\circledR}$, GSK Laval). Doses were separated by 21 days. Blood samples were obtained at baseline (day 0 ) and days 21 and 42 and tested for hemagglutination inhibiting antibodies to $\mathrm{pH} 1 \mathrm{~N} 1$ virus. Immune responses were examined by standard criteria.

RESULTS: In total 150 subjects had dose one (GrpA:76 , GrB:74) and 134 completed the study (89\%). Baseline parameters were similar between groups: $83 \%$ male, mean age 45, BMI 26, other health conditions in $75 \%$, detectable HIV RNA in $15 \%$ and detectable antipH1N1 (titer $\geq 10$ ) in $31 \%-34 \%$. After the first dose titers $\geq 40$ ("protective") were present in 76\% (GrpA) and $81 \%(\mathrm{GprB})$, increasing in 
GrpB to $94 \%$ after a second dose $(\mathrm{p}<0.001)$. Among GrpB subjects with no pre-existing antibody, titers $\geq 40$ were present in $76 \%$ after one dose and $91 \%$ after two doses. Geometric mean titers (GMT) were $130(\mathrm{GrpA})$ and $102(\mathrm{GrpB})$ after dose one and $156(\mathrm{GrpB})$ after dose two, while GrpA GMT declined in the interval to $97(p=0.06$, GrpA vs GrpB). At day 42, GMT ratios exceeded baseline by 11 fold in GrpA and 19 fold in GrpB ( $=0.05)$. No serious adverse events occurred after either vaccine dose.

CONCLUSIONS: One dose of adjuvanted influenza vaccine was quite immunogenic in HIV patients but a second "booster" dose significantly increased protection. Use of this adjuvanted vaccine and a "booster" dose represent an important approach to increasing protection in this vaccine hyporesponsive population.

\section{P117}

SURVEILLANCE DU SYNDROME DE GUILLAIN-BARRÉ (SGB) AU QUÉBEC LORS DE LA CAMPAGNE DE VACCINATION DE MASSE CONTRE L'INFLUENZA PANDÉMIQUE A/H1N1

\section{G Deceuninck ${ }^{1}$, P De Wals ${ }^{1}$, G De Serres ${ }^{1}$, E Toth $^{2}$}

${ }^{1}$ Quebec City, QC; ${ }^{2}$ Montréal, QC

Contexte: Une campagne de vaccination de masse avec le vaccin adjuvanté A/H1N1 a été réalisée au Québec durant l'automne 2009. Vu l'histoire de survenue du SGB en 1976 aux USA, un système de surveillance renforcé a été mis en place dès la début de la campagne.

OBJECTIF : Détecter de façon prospective une augmentation de l'incidence du SGB dans la population du Québec.

MÉTHODES : Le SGB a été ajouté à la liste des maladies à déclaration obligatoire et tous les neurologues invités à participer au signalement de tous les cas, vaccinés ou non.

De mi-octobre 2009 à mi-avril 2010, les fréquences hebdomadaires ont été comparées à un nombre moyen attendu dans deux groupes d'âge en ajustant la statistique de Poisson pour les comparaisons multiples. La méthode CUSUM a été utilisée pour détecter une hausse soutenue de la fréquence hebdomadaire $\geq 1$ par rapport à niveau de base de 2,5 cas, avec un délai d'alerte de 5 semaines et une probabilité de fausse alarme inférieures à $5 \%$ par semaine.

RÉSULTATS : Au total, 58 cas ont été répertoriés. La fréquence hebdomadaire a été comprise entre 0 et 3 chez les moins de 50 ans (seuil d'alarme $=5$ cas) et entre 0 et 4 chez les $<50$ ans (seuil $=6$ cas). Les analyses de CUSUM ont montré une hausse de fréquence dans les semaines suivant le pic de vaccination (qui a lui-même suivi le pic de la deuxième vague d'influenza) mais sans atteinte du seuil d'alerte.

CONCLUSION : La surveillance prospective n'a pas généré d'alarme et on peut écarter l'hypothèse d'un risque comparable à celui observé en 1976. Toutefois, la sensibilité des méthodes est faible pour des augmentations de risque de faible amplitude et d'autres analyses sont en cours pour analyser le risque en fonction de l'exposition au vaccin.

\section{P118 \\ IMPACT OF CO-MORBIDITIES ON OUTCOME SEVERITY AMONG PANDEMIC H1N1 2009 (pH1N1) HOSPITALIZED CASES IN CANADA}

\section{J Vachon, MT Do, R Rodin, J Pulickal, L Pelletier}

Ottawa, ON

BACKGROUND: The National Advisory Committee on Immunization (NACI) recommends annual influenza vaccination for adults and children with chronic medical conditions to reduce morbidity and mortality associated with influenza.

OBJECTIVES: To determine whether the presence of specific medical conditions increase the risk of severe outcomes for pH1N1 hospitalized patients and how those co-morbidities modify the risk for particular groups (e.g. pregnant women).

METHODS: Disease specific risk estimates were based on detailed information of all laboratory-confirmed pH1N1 hospitalized, IntensiveCare Units (ICU) and fatal cases from all provinces and territories in
Canada collected between April 18, 2009 and April 3, 2010. Patients admitted to hospital but not to the ICU were described as non-severe cases, while patients who were admitted to ICU or died were considered severe cases.

RESULTS: Of the 8,227 hospital admissions with pH1N1 during the study period, 6,653 were non-severe cases, 1,225 were admitted to ICU and 349 died while hospitalized. The presence of at least one preexisting co-morbidity (OR 4.2, CI 95\% 3.7-4.7) was associated with increased odds of severe outcome. Hospitalized cases with neurological, renal, heart, respiratory disease, diabetes or immunosuppression were all significantly associated with increased risk of severe outcome. While hospitalized children under 5 years of age (OR 3.1, CI 95\% 1.8-5.4) and 45 years of age and older (OR 2.0, CI 95\% 1.5-2.7) with at least one underlying medical condition had a higher risk of severe outcome compared to healthy individuals. The association was not statistically significant for pregnant women and Aboriginals.

CONCLUSIONS: Hospitalized pH1N1 patients with medical conditions are more at risk of severe outcome compared to those without. The risk of severe outcome among young children and people of 45 years of age and older is increased by the presence of co-morbidities. Cases with underlying neurological disease were found to be of highest risk for severe outcomes, especially among young children.

\section{P119}

COMPARISON OF THE AGE DISTRIBUTION AMONG HOSPITALIZED PANDEMIC H1N1 (pH1N1) CASES DURING THE FIRST AND SECOND WAVES IN CANADA

\section{MT Do, B Winchester, L Pelletier}

Ottawa, ON

BACKGROUND: Early studies of the $2009 \mathrm{H} 1 \mathrm{~N} 1$ pandemic in the Unites States and Mexico showed that people under 20 years of age were at higher risk of hospitalization than what is observed during seasonal influenza. Data from previous pandemics suggest that that a shift in the age distribution toward seasonal patterns will likely not occur for several years.

PURPOSE: To describe and compare age distributions of hospitalized pH1N1 infected cases during the two waves of the pandemic in Canada.

METHODS: Descriptive statistics were conducted on the national database of $\mathrm{pH} 1 \mathrm{~N} 1$ hospitalized cases during the first (April 18th to August 29th 2009) and second wave (including the post-peak period from August 30th to April 3rd 2010). Age-specific incidence ratios were used to compare risk for different age groups.

RESULTS: A total of 1,490 individuals were hospitalized with pH1N1 during the first wave and another 6,737 were hospitalized during the second. The mean age of hospitalized cases was 27 in wave $1 \mathrm{com}$ pared to 32 years in wave $2(\mathrm{p}<0.001)$. The age difference between the 2 waves remain after excluding individuals of Aboriginal origin $(\mathrm{p}<0.05)$. In the first wave, those under the age of 20 years of age made up of $43 \%$ of all hospitalized cases. This proportion decreased to $36 \%$ in the second wave. In both waves, the highest age-specific incidence ratios were among those under 20 years of age, particularly those under 5 years of age $(\mathrm{SIR}=4.16,95 \% \mathrm{CI}+0.46)$.

CONCLUSIONS: Even though there was a change in age distribution between the first and second waves, those under 20, particularly those under 5 years of age remained at highest risk of hospitalization. This finding may have some implications in the groups for whom the influenza vaccine is recommended in the coming years. 
P120

CANADIAN FAMILY PHYSICIANS' AND PAEDIATRICIANS' OPINIONS TOWARD A(H1N1) PANDEMIC VACCINE BEFORE AND AFTER VACCINATION CAMPAIGN ONSET E Dubé $^{1}$, V Gilca ${ }^{1}$, C Sauvageau ${ }^{1}$, N Boulianne ${ }^{1}$, FD Boucher ${ }^{1}$, JA Bettinger ${ }^{2}$, S McNeil ${ }^{3}$, I Gemmill ${ }^{4}$, F Defay ${ }^{1}$, F Lavoie $^{1}$ ${ }^{1}$ Quebec, QC; ${ }^{2}$ Vancouver, BC; ${ }^{3}$ Halifax, NS; ${ }^{4}$ Kingston, ON BACKGROUND: In June 2009, WHO declared an influenza A(H1N1) pandemic. In October 2009, the largest vaccination campaign in Canadian history began.

PURPOSE: To compare family physicians' and paediatricians' knowledge, attitudes and practices (KAP) regarding $\mathrm{A}(\mathrm{H} 1 \mathrm{~N} 1)$ vaccination before and after the beginning of the campaign.

METHODS: Three mailings of a self-administered questionnaire were done from August to November 2009 to a random sample of family physicians and to all paediatricians. Questionnaires were analyzed in two subsets: before versus after the beginning of vaccination campaign (October 29). Logistic regression analyses of respondents' intention to recommend the $\mathrm{A}(\mathrm{H} 1 \mathrm{~N} 1)$ vaccine were computed.

RESULTS: Overall participation rate was 43\% (1283/2959). Socioprofessionals characteristics of both groups were comparable. Before the beginning of the campaign, $60 \%$ of respondents perceived $\mathrm{A}(\mathrm{H} 1 \mathrm{~N} 1)$ pandemic influenza as serious comparatively to $75 \%$ after $(\mathrm{p}<0.0001)$. Before vaccination campaign, few respondents agreed or strongly agreed about $\mathrm{A}(\mathrm{H} 1 \mathrm{~N} 1)$ vaccine safety $(48 \%)$ and effectiveness $(34 \%)$ comparatively to $78 \%$ and $74 \%$ after $(\mathrm{p}<0.0001)$. Perceived acceptability of $\mathrm{A}(\mathrm{H} 1 \mathrm{~N} 1)$ vaccine by the public was comparable ( $43 \%$ versus $40 \%$ ), but perceived acceptability by the vaccinators increased from $69 \%$ to $85 \%(p=0.0004)$. Self-estimated sufficiency of information received on $\mathrm{A}(\mathrm{H} 1 \mathrm{~N} 1)$ vaccine raised from $30 \%$ to $78 \%(\mathrm{p}<0.0001)$. Intention to recommend $\mathrm{A}(\mathrm{H} 1 \mathrm{~N} 1)$ vaccine to their patients increased from $78 \%$ to $90 \%(\mathrm{p}<0,0001)$. Main determinant of respondents' intention to recommend the vaccine were: intention to get themselves the $\mathrm{A}(\mathrm{H} 1 \mathrm{~N} 1)$ vaccine and, after the beginning of the campaign, perceived usefulness of vaccines recommended by public health authorities.

DISCUSSION: Dissemination of information regarding the safety and immunogenicity of $\mathrm{A}(\mathrm{H} 1 \mathrm{~N} 1)$ vaccine as well as official recommendations have enhanced the confidence of family physicians and paediatricians regarding this vaccine and have had a positive impact on their intention to recommend it.

\section{P121}

VACCINATION CONTRE LA GRIPPE A(H1N1): CONNAISSANCES, CROYANCES ET ATTITUDES DES QUÉBÉCOIS(ES)

E Dubé $^{1}$, M Guay ${ }^{1,2}$, N Boulianne ${ }^{1}$, C Sauvageau ${ }^{1}$, M Landry $^{3}$,

F Markowski $^{3}$, F Defay ${ }^{1}$, M Kiely ${ }^{1}$, P Clément ${ }^{3}$

${ }^{1}$ Québec, QC; ${ }^{2}$ Sherbrooke, QC; ${ }^{3}$ Montréal, QC

CONTEXTE : Au Québec, la campagne de vaccination contre la grippe $\mathrm{A}(\mathrm{H} 1 \mathrm{~N} 1)$ a débuté le 26 octobre 2009 et près de $60 \%$ de la population a été vaccinée.

BUT : Connaître les déterminants psychosociaux de la vaccination contre la grippe $\mathrm{A}(\mathrm{H} 1 \mathrm{~N} 1)$ des adultes québécois.

MÉTHODES : Les données ont été recueillies lors d'une enquête téléphonique en mai 2010 en utilisant le modèle des croyances relatives à la santé. Les participants étaient recrutés à partir d'une liste de numéros de téléphone générés aléatoirement. Des analyses descriptives et comparatives (vaccinés vs non vaccinés) ont été effectuées.

RÉSULTATS : Au 10 juin 2010, 4638 adultes avaient répondu au questionnaire. Au moment de l'enquête, $54 \%$ se considéraient très bien informés sur la grippe $\mathrm{A}(\mathrm{H} 1 \mathrm{~N} 1)$. 67\% des personnes vaccinées considéraient la grippe $\mathrm{A}(\mathrm{H} 1 \mathrm{~N} 1)$ comme une maladie dangereuse comparativement à $39 \%$ des non vaccinés $(\mathrm{p}<0,0001)$. À l'automne 2009, les personnes ayant été vaccinées se considéraient plus à risque d'attraper la grippe $\mathrm{A}(\mathrm{H} 1 \mathrm{~N} 1)$ que celles n'ayant pas reçu le vaccin
( $41 \%$ vs $18 \%$ p $<0,0001)$. Davantage de personnes vaccinées que non vaccinées croyaient en l'efficacité du vaccin (30\% vs 20\% p<0,0001), mais plusieurs demeuraient incertains à ce sujet (environ 14\% au total). Les personnes vaccinées étaient toutefois plus convaincues de la sécurité du vaccin que les non vaccinées ( $89 \%$ vs $46 \%$ p<0,0001). La moitié des personnes vaccinées et $23 \%$ des personnes non vaccinées avaient reçu une recommandation de se faire vacciner par un professionnel de la santé. Près de la moitié $(49 \%)$ des personnes non vaccinées croyaient que le vaccin contre la grippe $\mathrm{A}(\mathrm{H} 1 \mathrm{~N} 1)$ pouvait causer la grippe, versus $23 \%$ des vaccinés $(p<0,0001)$.

CONCLUSIONS : La perception des risques de la grippe et la sécurité des vaccins sont des facteurs importants sur lesquels il faudrait mettre l'accent lors de la promotion de la vaccination saisonnière.

\section{P122}

\section{A COST ANALYSIS OF THE EFFICIENCY OF H1N1 MASS IMMUNIZATION CLINICS}

\section{JK Fediurek, J Tober, E Aly}

Brantford, ON

BACKGROUND: The Canadian response to the H1N1 pandemic included the delivery of mass immunization clinics. The organization of these clinics demanded maximizing the use of finite human resources and vaccine supply while also maximizing the volume of clients immunized. An online client appointment system was implemented to automate client bookings for $\mathrm{H} 1 \mathrm{~N} 1$ vaccination. Clients could access the system from the internet and were able to self schedule an appointment for a convenient time and location. The number of appointment slots was regulated based on staffing and vaccine availability. An electronic documentation system was used to facilitate client entry and minimize the time required for nursing documentation.

PURPOSE: To evaluate the cost effectiveness and efficiency of H1N1 mass immunization clinics.

METHODS: A financial analysis was conducted to assess the costs of delivery of $\mathrm{H} 1 \mathrm{~N} 1$ vaccine at multiple simultaneous community clinics. Total costs for delivery of clinics were calculated and compared to client volumes.

RESULTS: A total of 36,636 doses of H1N1 vaccine were delivered through 136 community clinics by local health unit staff. The average time for delivery of vaccine was 5 minutes per dose per nurse for a total time for clients of 20 minutes, including a 15 minutes post immunization wait time. At a Ministry reimbursement of $\$ 10.00$ per dose, 38 clients per hour was a cost break even point when salary, supplies, facility costs and miscellaneous expenses were taken into account. Clinics operated at $100+\%$ capacity with a through put of 60 clients per hour when fully functional.

CONCLUSIONS: Use of an online appointment system demonstrated a cost effective strategy for efficient scheduling of staff and provision of immunizations based on vaccine supply. An electronic documentation system enhanced the ability to provide mass immunization clinics in a manner which maximized client volume and minimized client wait and procedure times.

\section{P123}

\section{PREDICTORS OF pH1N1 VACCINE UPTAKE}

J Foisy, R Sanderson, LC Rosella, NS Crowcroft Toronto, ON

BACKGROUND: Many countries implemented targeted pH1N1 vaccination programs, however; the Canadian government stated that the vaccine would be available to anyone who wished to be vaccinated. Nonetheless, vaccine uptake varied across the population. Identification of predictors of uptake, including reasons for refusal, help evaluate the program, craft appropriate messages and identify under-represented populations.

PURPOSE: To identify determinants of $\mathrm{pH} 1 \mathrm{~N} 1$ vaccination among a population in Ontario, Canada. 
METHODS: The Rapid Risk Factor Surveillance System (RRFSS) is an ongoing, random digit-dialing telephone survey of the adult population in Ontario, used to gather surveillance data, monitor public opinion and collect key public health information. This survey infrastructure was used to collect data on vaccination status, predictors of uptake, as well as reasons for declining vaccination. Data were described using unweighted frequencies and relationships with $\mathrm{pH} 1 \mathrm{~N} 1$ vaccine uptake were derived using logistic regression.

PRELIMINARY RESULTS: A total of 9010 Ontario residents participated between January 14 and May 4, 2010. Approximately 40\% reported receiving the $\mathrm{pH} 1 \mathrm{~N} 1$ vaccine and $39 \%$ reported having at least one child vaccinated. Predictors for being vaccinated identified in the multivariate logistic regression include age (odds ratio [OR] per 10 years, 1.08 ; $\mathrm{CI}, 1.04$ to 1.12 ), female (OR, 1.17; CI, 1.06 to 1.29 ), children in the household (OR, 1.29; CI, 1.14 to 1.46), and receipt of the 2008/09 seasonal influenza vaccine (OR, 9.38; CI, 8.43 to 10.44). Among the unvaccinated, the leading reasons for refusal were the disbelief one was at risk for $\mathrm{pH} 1 \mathrm{~N} 1$ (45\%), concern with vaccine safety (17\%) and perceived lack of research or testing (16\%).

CONCLUSION: Several factors are associated with receiving $\mathrm{pH} 1 \mathrm{~N} 1$ vaccine. Ongoing dialogue between the public and decision makers is an important way to ensure a successful immunization campaign. Results from this population-based study show that vaccine campaigns should focus on the perceived risk of infection, research and safety of the vaccine.

\section{P124}

\section{CLINIC STAFF PERCEPTIONS OF DATA COLLECTION METHODOLOGIES USED DURING THE 2009 H1N1 INFLUENZA CAMPAIGN IN CANADA}

J Foisy ${ }^{1}$, CL Heidebrecht ${ }^{1}$, JA Pereira ${ }^{1}$, S Quach ${ }^{1}$, SD Quan ${ }^{1}$, $M_{\text {Guay }}{ }^{2}$, JA Bettinger ${ }^{3}$, SL Deeks ${ }^{1}$, JC Kwong ${ }^{1}$

${ }^{1}$ Toronto, ON; ${ }^{2}$ Longeuil, QC; ${ }^{3}$ Vancouver, BC

BACKGROUND: During the 2009 H1N1 immunization campaign, electronic and paper methods were employed to collect client-level vaccination data in clinics across Canada. The campaign offered an opportunity to study the usability of the various data collection methods.

PURPOSE: To identify staff perceptions regarding the usability of data collection methods employed at their H1N1 clinics.

METHODS: A convenience sample of clinic staff working in public health agencies and hospitals in 9 provinces/territories across Canada completed a questionnaire adapted from the IBM Computer System Usability Questionnaire, in which they indicated their level of agreement with seven statements regarding the usability of the data collection method they used at their vaccination clinic. Questions included overall ease of use, effectiveness of the method utilized, efficiency at completing tasks, comfort using the method, ability to recover from mistakes, ease of learning the method and overall satisfaction with the method. A 5 point Likert-like scale was used to measure responses.

RESULTS: Most respondents (96\%) were employed in sites run by public health. Respondents included 186 nurses and 114 administrative staff, among whom $90 \%$ and $47 \%$, respectively, used a paper method for data collection. Approximately half the respondents had a year or less of experience with immunization-related tasks during seasonal influenza campaigns. Over $90 \%$ of all frontline staff found their data collection method easy to use, perceived it to be effective in helping them complete their tasks, felt quick and comfortable using the method, and found the method easy to learn, regardless of whether a hybrid or electronic system was used.

CONCLUSION: Frontline staff using electronic and paper-data collection methods had similarly favourable perceptions of their method's usability, indicating that this factor may not be a barrier for adopting new technologies.
P125

HCW H1N1 IMMUNIZATION RATES SOAR TO NEW HEIGHTS IN NEWFOUNDLAND LABRADOR MM Yetman' ${ }^{1}$ D Moralejo'1, P March'2, L Mitchell ${ }^{2}$, P Price ${ }^{3}$, J Young ${ }^{3}$, P Ralph ${ }^{4}$, S Woolfrey ${ }^{4}$, D Ronayne ${ }^{1}$, A Fowler ${ }^{1}$ ${ }^{1}$ St John's, NL; ${ }^{2}$ Goose Bay, NL; ${ }^{3}$ Corner Brook, NL; ${ }^{4}$ Grand Falls-Windsor, NL

BACKGROUND: Multi-pronged strategies aimed at increasing healthcare worker (HCW) uptake of annual seasonal influenza vaccine have had limited success, with rates usually between 10-60\% in Newfoundland Labrador (NL). In May 2009, with H1N1 rapidly approaching, the Infection Prevention \& Control (IPAC)-Occupational Health Hygiene $(\mathrm{OHH})$ subcommittee of the Provincial Pandemic Steering Committee identified its top priority as education to promote both vaccination and use of appropriate infection control precautions.

METHOD: A standardized one hour presentation was developed and used in formal education sessions by IPAC-OHH. Seven PowerPoint mini-lectures were also developed. Attendance was mandatory in some regions. Three were specific to influenza for HCWs unable to attend a presentation: Seasonal and Pandemic Influenza, Pandemic Influenza Precautions and Influenza Vaccination. Four mini-lectures reinforced measures to prevent spread of infection: Chain of Infection, Point-ofCare Risk Assessment, Routine Practices, and Additional Precautions. All seven mini-lectures were made available on DVD, on the government's web site and via the facilities' intranets.

RESULTS: Written feedback was obtained from $1600 \mathrm{HCWs}$ and 850 nursing students. Over $95 \%$ agreed or strongly agreed that their knowledge and their confidence increased related to precautions to take for a patient with influenza-like-illness (ILI), strategies to prevent transmission, and self-assessment/self care for ILI. About $75 \%$ said that they planned to receive the pandemic vaccine. Of those who had not previously received seasonal influenza vaccine, $37 \%$ said they planned to receive the pandemic vaccine and $41 \%$ were considering it. The actual uptake of H1N1 vaccine by HCWs in NL reached $100 \%$ in three Regional Health Authorities and 82\% in the fourth.

CONCLUSION: An educational program delivered to HCWs in $\mathrm{NL}$ prior to the H1N1 immunization campaign was associated with increased uptake. Collaboration between IPAC-OHH professionals in preparing educational materials for HCWs facilitated delivery of key and consistent messages across the province. Additionally the use of multi-media strategies allowed HCWs independence in choosing the educational format best suited to them.

\section{P126}

ATTITUDES ET CROYANCES DES FEMMES ENCEINTES VISÀ-VIS DE LA VACCINATION CONTRE L'INFLUENZA A/H1N1

\section{P Fabry, A Gagneur, J Pasquier}

\section{Sherbrooke, QC}

CONTEXTE : Lors de la pandémie d'influenza A/H1N1 les femmes enceintes ont été vaccinées en priorité en raison des risques de complications. Au Québec, 63\% des femmes enceintes ont été vaccinées, ce qui est supérieur aux taux de vaccination contre l'influenza saisonnière. Cependant, le comportement des femmes enceintes vis-à-vis de la vaccination en situation de pandémie est mal connu.

BUT : Notre objectif est d'identifier les facteurs influençant la prise de décision des femmes enceintes vis-à-vis de la vaccination.

MÉTHODES : Une enquête a été réalisée en février 2010 auprès de femmes enceintes ou en post-partum récent au CHUS à l'aide d'un questionnaire autoadministré basé sur le Health Belief Model (HBM). Ont été recueillis les données sociodémographiques, le statut vaccinal, les sources d'informations consultées, les connaissances sur la vaccination, et l'exploration des dimensions de l'HBM : efficacité et risque de la vaccination, gravité et vulnérabilité vis-à-vis de l'influenza. 
L'association entre les variables du questionnaire et le statut vaccinal a été évaluée par analyses univariées et multivariées.

RÉSULTATS : Sur les 250 femmes interrogées, $95 \%$ savaient que la vaccination était recommandée, mais seulement $76 \%$ ont bénéficié $\mathrm{du}$ vaccin. Les variables associées positivement à la vaccination sont la vaccination tardive au cours de la grossesse $(\mathrm{OR}=7,3$; IC95\% 2,1 $25,3)$, la croyance en l'efficacité du vaccin ( OR = 7; IC95\% 2-23,4), la consultation du site web pandemie-quebec $(\mathrm{OR}=4,5$; IC95\% 1,5 - 13,4). En revanche, la croyance que le vaccin n'a pas été suffisamment testé $(\mathrm{OR}=0,08$; IC95\% 0,02-0,35) et la consultation de sites web grand public $(\mathrm{OR}=0,22$; IC95\% 0,06-0,81) sont associées à un moindre taux de vaccination.

CONCLUSIONS : La majorité des femmes enceintes connaissaient les recommandations par rapport à la vaccination. Internet a joué un rôle important dans leur décision de se faire vacciner. Une meilleure information sur la sécurité du vaccin doit être préparée pour les prochaines pandémies.

\section{P127 \\ SHORT AND LONG-TERM ACTIVE ELECTRONIC SURVEILLANCE OF A LARGE NUMBER OF HEALTHCARE WORKERS FOLLOWING ADMINISTRATION OF ADJUVANTED pH1N1 VACCINE \\ M Gariépy ${ }^{1}$, G De Serres ${ }^{1}$, I Rouleau ${ }^{1}$, B Coleman ${ }^{2}$, \\ K Wilkinson ${ }^{3}$, N Ouhoumanne ${ }^{1}$, M Benoit ${ }^{1}$, A Ambrose ${ }^{4}$, \\ S McNeil ${ }^{4}$, A McGeer ${ }^{2}$ \\ ${ }^{1}$ Quebec, QC; ${ }^{2}$ Toronto, ON; ${ }^{3}$ Ottawa, ON; ${ }^{4}$ Halifax, NS}

BACKGROUND: The active surveillance of a large group of health care workers $(\mathrm{HCW})$ vaccinated with the adjuvanted monovalent pH1N1 influenza vaccine was conducted through a web-based survey. METHODS: All HCW immunized in one of three hospitals (Quebec City, Halifax and Toronto) were invited to participate in a web-based active surveillance of vaccine safety. The main outcome $(\mathrm{MO})$ was the onset after vaccination of any new health problem or the exacerbation of an existing condition that was severe enough to cause work absenteeism or require medical consultation. Participants were contacted 7 , 14 and 28 days after vaccination by email and provided with a hyperlink to a secure website for a brief survey. One site which enrolled $79 \%$ of all participants also did a 6 month follow-up. Participants reporting $\mathrm{MO}$ were contacted by a nurse for detailed information.

RESULTS: Among the 6,242 $\mathrm{HCW}$ s enrolled in the study, $80 \%$ $(4,979)$ responded to at least one of the three first surveys and 3,275 (52\%) at all first three timepoints: 507 reported at least one MO. The proportion reporting $\mathrm{MO}$ varied from $5.6 \%(242 / 4,307)$ in the first 7 days after vaccination, to $4.7 \%(200 / 4,264)$ at 14 days, and $4.8 \%$ $(206 / 4,028)$ at 28 days. MO caused work absenteeism in 55\%, required a medical consultation in $20 \%$ and resulted in both absenteeism and medical consultation in $26 \%$. Local reactions sufficient to cause absenteeism or a medical consultation occurred in $\sim 1$ in 200 vaccinees. Three patients ( 1 in 2000 vaccinees) reported an allergic reaction with onset within 24 hours. Among the $4812 \mathrm{HCW}$ who were sent the survey for the 6 months follow-up, 3043 (63\%) responded; 59 reported a hospitalization or a serious adverse event. None reported the same AE suggesting no association with the vaccine

CONCLUSIONS: Despite suboptimal response rate, this active surveillance of $\mathrm{HCW}$ gathered information on a large number of participants. This vaccine was associated with local and allergic-like reactions but no serious long-term AE was detected.
P128

\section{APPROACHES TO IMMUNIZATION DATA COLLECTION EMPLOYED ACROSS CANADA DURING THE H1N1 VACCINATION CAMPAIGN}

CL Heidebrecht ${ }^{1}$, JA Pereira ${ }^{1}$, S Quach ${ }^{1}$, J Foisy ${ }^{1}$, S Quan ${ }^{1}$, M Finkelstein', SL Deeks', M Guay², JA Bettinger ${ }^{3}$,

NS Crowcroft ${ }^{1}$, F Kolbe ${ }^{1}$, JC Kwong ${ }^{1}$

${ }^{1}$ Toronto, ON; ${ }^{2}$ Longueuil, QC; ${ }^{3}$ Vancouver, BC

BACKGROUND: A critical component of the 2009 H1N1 pandemic vaccination campaign was the collection of immunization data at the point of care. To meet reporting requirements and to ensure timely availability of coverage information for assessment of the public health response, many jurisdictions across Canada employed new or modified approaches to vaccine data collection.

PURPOSE: To observe and characterize the range of influenza immunization data collection approaches used across Canada.

METHODS: The research team visited immunization clinics as part of a multi-stage observational study between October and December, 2009. To supplement the time and survey data collected, clinic processes - particularly tasks related to data collection and management - were observed at each study site. Tasks included registration, medical history collection and review, vaccine record-keeping, vaccination proof preparation, and data entry. Field notes were analyzed in order to understand the data collection mechanisms that comprised each information system as a whole. The study team's interpretation of the data collection mechanisms were summarized and sent to each organization for validation.

RESULTS: 79 clinic sites representing 38 organizations in nine provinces and territories were observed. Data collection approaches were grouped into two categories: electronic systems (9/38), in which all data are captured on computer at the point of vaccination; and hybrid systems (29/38), comprised of both computerized and paper-based data collection tasks. Observed systems included standalone databases, immunization registries, and electronic health records. Organizations incorporated components such as magnetic card reader technology, telephone registration, and pre-populated fields into data collection approaches.

CONCLUSION: Canadian jurisdictions employed different data collection approaches during the H1N1 vaccination campaign. System characteristics can have important implications for on-site efficiency and organization as well as program planning and evaluation. The systems observed have been described in detail to allow vaccine providers and planners to learn from what has been done elsewhere.

\section{P129}

OVERCOMING INEQUITIES - REACHING HARD TO REACH POPULATIONS DURING H1N1

\section{AC Henteleff, D Heywood, C Kurbis, H Kris}

\section{Winnipeg, MB}

BACKGROUND: Mass Immunization clinics have been proven to be an efficient method for immunizing large numbers of people in a compressed time period. During the planning phases of pandemic preparation it was identified that certain populations would likely be disproportionally impacted by the pandemic due to both existing inequalities in health and the more limited capacities of under-resourced communities. These populations have poor immunization rates in general and are therefore less likely to access immunization in mass clinic settings.

PURPOSE: Plan and implement a strategy to immunize hard-to-reach populations.

METHODOLOGY: A working group of public health staff experienced in service provision to hard-to-reach populations developed an "equities strategy" to overcome systemic barriers to immunization and bring "mass immunization" to hard to reach populations. The equities strategy, woven into the entire pandemic plan, was built on community 
capacity and engaged multiple programs, staffing, community partners and venues. Asking the question 'What inequities exist?', frontline, program and community staff were able to identify 14 distinct groupings of hard-to-reach populations acknowledging that there is overlap between some groups and individuals. Examples include street involved, those with drug or alcohol dependence, the home bound, those with language barriers etc.

RESULTS: With careful assessment and planning, a multifaceted approach was used to improve access to pandemic vaccine for hard to reach populations. This strategy included improving access at mass clinics, community outreach clinics in venues both familiar and unfamiliar with immunization services, enhanced home bound immunization services, enhanced interpreter and translation services, use of grass roots communication methods within hard-to-reach populations, and providing immunization along with other service provision.

CONCLUSION: Commitment to an equities strategy created an environment that allowed for creative use of staffing, community and existing services to overcome systematic barriers to immunization in hard-to-reach populations. Lessons learned from this approach will serve to provide a better foundation on which to improve immunization services in general to hard-to-reach populations in the future.

\section{P130 \\ PERSISTENT PAIN IN THE VACCINATED LIMB FOLLOWING THE 2009-10 PANDEMIC H1N1 MASS IMMUNIZATION CAMPAIGN IN CANADA}

\section{N Ahmadipour, S Houle, S Kim, J Lafleche, R Pless, B Law Ottawa, ON}

BACKGROUND: Pain at the injection site is a known feature of adjuvanted pandemic H1N1 (pH1N1) vaccines, but usually lasting only a few days. There were reports of persistent pain at the injection site and/or the ipsilateral limb during the recent pandemic influenza vaccination campaign.

PURPOSE: To review reports describing persistent pain or decreased joint range of motion (DJROM) involving the vaccinated limb following $\mathrm{pH} 1 \mathrm{~N} 1$ vaccines.

METHODS: Reports of Adverse Events Following Immunization (AEFIs) are forwarded to the Public Health Agency of Canada by provinces and territories. Reports indicating pain or DJROM symptoms were extracted from the Canadian Adverse Event Following Immunization Surveillance System database. The main variables included symptoms, duration, age, gender, time to symptom onset, level of care obtained and outcome.

RESULTS: Of 4,353 reports received, 515 indicated at least one pain/DJROM symptom. Time to onset was 1 minute to 49 days postvaccination (mean 1.5 days). Just over half reported duration to resolution: $<2$ days in $20 \%, 2-7$ days in $52 \%, 8-30$ days in $21 \%$, and $>30$ days in $7 \%$. In the remaining $47 \%$, either a symptom persisted at the time of reporting, or duration to resolution was not mentioned. The outcome was reported as not recovered in 34\% and unknown in 16\%. Fifty-eight percent obtained care, mainly non-urgent visit (34\%).

CONCLUSION: Although pain at the injection site is relatively common following any vaccine, it appears that after adjuvanted pH1N1 vaccines it was more severe and in some cases affected the entire limb for an extended period. In the literature, injection technique is described as one possible cause, suggesting that vaccination guidelines ensure describing the correct site of vaccination. Cases of unusually long duration may require detailed specialist follow up to ascertain the reason for persistent pain.
P131

THE SAFETY PROFILE OF PANDEMIC H1N1 VACCINES: REPORTS OF ADVERSE EVENTS FOLLOWING IMMUNIZATION (AEFI) RECEIVED BY THE PUBLIC HEALTH AGENCY OF CANADA (PHAC), OCTOBER 2009 THROUGH MARCH 2010

\author{
Lafleche, N Ahmadipour, H Anyoti, R Pless, B Law \\ Ottawa, ON
}

BACKGROUND: The authorization for sale of pandemic H1N1 (pH1N1) vaccines was based on more limited data than required for full licensure. Enhanced post-marketing safety surveillance activities were both required and of paramount importance.

PURPOSE: To describe the profile of Adverse Events Following Immunization $(\mathrm{AEFI})$ with $\mathrm{pH} 1 \mathrm{~N} 1$ vaccines based on case reports received by PHAC, and outline safety issues arising from surveillance.

METHODS: AEFI reports for $\mathrm{pH} 1 \mathrm{~N} 1$ vaccines in the Canadian Adverse Event Following Immunization Surveillance System database were extracted. Vaccine recipient demographic, vaccine(s) administered, AEFIs coded, severity and patient outcomes were among the variables assessed. A serious adverse event is one that resulted in or prolonged hospitalization, death, permanent disability, and/or was life threatening.

RESULTS: A total of 6,400 reports of AEFI for $\mathrm{pH} 1 \mathrm{~N} 1$ vaccines were reported, for a reporting rate of close to 50 per 100,000 doses administered. 271 were classified as serious ( 2.2 per 100,000 ). 119 Hospitalizations were reported (0.94), along with; 19 Deaths (0.2), 31 cases of Guillain-Barré Syndrome (0.2) and 135 cases of Anaphylaxis (1.08). The reporting rate was much higher than the previous 2 influenza seasons but closer to the 2000-2002 seasons when the Oculo-Respiratory Syndrome signal emerged. The majority $(55 \%)$ of reports involved adults. Twice as many reports were for female vaccine recipients, but the proportion decreased to 1.6:1 for serious reports. For AEFIs reported, $41 \%$ described allergic reactions, while $7.9 \%$ were anaphylaxis. Neurologic events and injection site reactions were the next most common.

CONCLUSIONS: The focus by public health on the immunization campaign resulted in increased vigilance, generating the highest reporting rate for a vaccine in Canada. Reports of allergic-type reactions including anaphylaxis dominated the AEFI profile and formed an early safety signal. Detecting signals as they occur and providing a comprehensive overview of the safety profile following the season, are key elements ensuring confidence in similar future immunization programs.

\section{P132}

ENHANCING SURVEILLANCE OF ADVERSE EVENTS FOLLOWING IMMUNIZATION (AEFI) DURING THE 2009-2010 PANDEMIC INFLUENZA VACCINATION CAMPAIGN IN CANADA: PROCESSES AND OUTCOME

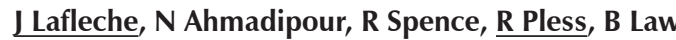 Ottawa, ON}

BACKGROUND: The fall of 2009 saw the largest immunization campaign in recent Canadian history, primarily with a monovalent H1N1 influenza vaccine containing an adjuvant not used in Canada. Public concerns over vaccine safety along with heightened vigilance by public health and health care providers meant that safety surveillance had to be scaled up.

PURPOSE: To review the components put in place for the enhanced surveillance of safety, describe the scope of AEFI reported in order to highlight the workflow and workload, outline implications on the ensuing safety monitoring of the H1N1 vaccines, and indicate lessons learned for future influenza vaccination seasons and routine safety monitoring of all vaccines.

METHODS: Weekly cumulative numbers of AEFIs were provided by each $\mathrm{P} / \mathrm{T}$ with the actual case reports to follow as soon as possible. Data on cases received by the end of the campaign were extracted from 
the Canadian Adverse Event Following Immunization Surveillance System (CAEFISS) database for analysis. Lessons learned regarding AEFI surveillance in Canada were discussed by a P/T working group.

RESULTS: More than 6,400 case reports of AEFI were notified by the $\mathrm{P} / \mathrm{Ts}$. Reporting peaked during the more active days of the campaign with over 100 reports sent or faxed to PHAC on a daily basis. While throughout the campaign the number of AEFIs received was less than half that of what had been indicated during weekly cumulative updates, serious reports were submitted on a timely basis and kept pace with the numbers anticipated. Nevertheless, final case reports were received well after conclusion of vaccinations. Several lessons thus emerged.

CONCLUSIONS: CAEFISS received more reports in less than 6 months for a single set of vaccines than is normally reported for all vaccines during any given year. This large number is impossible to completely process for real time data analysis, highlighting the need to employ various strategies of pharmacovigilance in order to help ensure vaccine safety. The lessons learned for future vaccine safety surveillance and strategies to overcome some limitations are outlined.

\section{P133}

IMMUNOGENICITY AND SAFETY OF AN A/H1N1V 2009 PANDEMIC INFLUENZA VACCINE ADMINISTERED WITH AND WITHOUT AS03 ADJUVANT IN CHILDREN AGED 6 MONTHS TO LESS THAN 9 YEARS

M Langley ${ }^{1}$, D Reich ${ }^{2}$, N Aggarwal ${ }^{3}$, D Connor ${ }^{4}$, M Lebel $^{5}$,

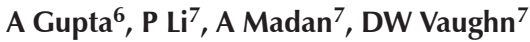

${ }^{1}$ Halifax, NS; ${ }^{2}$ Sudbury, ON; ${ }^{3}$ Brampton, ON; ${ }^{4}$ Winnipeg, MB;

${ }^{5}$ Montreal, QC; ${ }^{6}$ Toronto, ON; ${ }^{7}$ King of Prussia, PA

BACKGROUND: During the 2009 influenza A H1N1 pandemic children had high rates of illness and hospitalizations. Although children $<9$ years (yrs) receive 2 doses of seasonal trivalent inactivated vaccine $(\mathrm{V})$ in the 1 st year they are immunized, it was not known if this was required with a dose-sparing adjuvanted $(\mathrm{adj}) \mathrm{V}$ with a pandemic strain.

PURPOSE: To assess the immunogenicity and safety of different doses of adj and non-adj monovalent pandemic $\mathrm{Vs}_{\mathrm{s}}$ in this age group.

METHODS: 258 children in stable health were randomized to one of four regimens: $3.75 \mu \mathrm{g}$ A/California/7/2009 H1N1 influenza hemagglutinin A (HA) antigen plus ASO3 [11.86mg tocopherol/0.5mL dose],

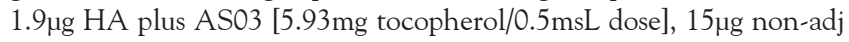
HA or 7.5 $\mu$ g non-adj HA on Days 0 and 21. Hemagglutination inhibition (HI) antibody titers were measured prior to Day 0 , and on Days 21 and 42. Adverse events (AEs) were monitored for 21 days and reactogenicity for 7 days after each dose.

RESULTS: After dose one, all groups fulfilled CHMP immunogenicity criteria for adults aged 18-60 years (seroconversion rate [SCR] $>40 \%$, seroprotection rate $[\mathrm{SPR}]>70 \%$, geometric mean fold rise

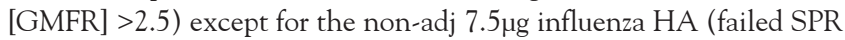
criterion). At Day 42, SPRs were 100\% across all age strata for both adj and $15 \mu \mathrm{g}$ non-adj. groups. SCR, SPR and GMFR were higher for adj than non-adj regimens. Injection site pain was the most common local $\mathrm{AE}$, with no increase post dose 2 . Irritability and fatigue were the most common general symptoms in children $<6$ yrs and $\geq 6$ yrs respectively. No clinically significant differences in frequency of unsolicited or medically attended AEs between groups was seen.

CONCLUSIONS: Novel pandemic influenza (A/H1N1 2009) vaccine formulations containing the dose-sparing ASO3 adj elicited higher SPRs, SCRs and GMFRs based on HI antibody titers than nonadj formulations in children aged 6 mos to $<9$ yrs, with acceptable safety profiles. One dose of all formulations except the $7.5 \mu \mathrm{g}$ non-adj HA V was adequate to satisfy CHMP criteria for immunogenicity.

\section{P134}

H1N1 INFLUENZA VACCINE COVERAGE IN CANADA DURING THE 2009-10 PANDEMIC

\section{Laroche, A Frescura, L Belzak \\ Ottawa, ON}

BACKGROUND: The largest immunization campaign in Canadian history was rolled out across all Canadian provinces and territories beginning in October 2009. To monitor H1N1 vaccine uptake in the Canadian population, jurisdictions were requested by the Canadian Immunization Committee to submit $\mathrm{H} 1 \mathrm{~N} 1$ vaccine uptake data to the Public Health Agency of Canada.

PURPOSE: To present vaccine coverage results from the 2009-2010 H1N1 influenza immunization campaign and to examine issues associated with $\mathrm{H} 1 \mathrm{~N} 1$ vaccine coverage reporting during the 2009-2010 pandemic.

METHODS: At the beginning and during the peak of the response, provinces and territories were asked to submit weekly aggregate vaccine uptake reports. The reports included information about age, sex, aboriginal status, by HINI vaccine priority group in each jurisdiction. National H1N1 vaccine coverage estimates were computed using denominators obtained from the jurisdictions and demographic tables based on Statistics Canada data. Factors influencing H1N1 vaccine coverage reporting and overall H1N1 vaccine uptake was examined.

RESULTS: As of June 1, 2010, complete reporting was received from 12 of 13 provinces and territories, percent coverage in the general population was $37.4 \%$ for individuals 6 months of age and older; however, coverage increased to $47.7 \%$ when the jurisdiction with incomplete reporting was excluded from analysis. Cumulative uptake of the H1N1 vaccine was highest for those with chronic medical conditions $(93.4 \%)$, followed by health care workers $(55.12 \%)$ and children aged 6 months to 4 years (47.4\%). In pregnant women, H1N1 vaccine coverage was estimated at $34.2 \%$.

CONCLUSION: Based upon reported doses administered in jurisdictions reporting completed data, approximately $47.7 \%$ of the general population was immunized with the H1N1 vaccine during the 2009. 2010 immunization campaign. H1N1 vaccine uptake was considerably higher in some priority groups. Delays in obtaining provincial and territorial data likely led to an underestimate of the proportion of Canadians immunized with the H1N1 vaccine.

\section{P135}

\section{WERE HEALTHCARE WORKERS AT INCREASED RISK OF PANDEMIC H1N1 INFECTION?}

SP Kuster ${ }^{1}$, S McNeil ${ }^{2}$, G De Serres ${ }^{3}$, M Loeb ${ }^{4}$, J Johnstone ${ }^{4}$, A McGeer ${ }^{1}$, B Coleman ${ }^{1}$

${ }^{1}$ Toronto, ON; ${ }^{2}$ Halifax, NS; ${ }^{3}$ Quebec, QC; ${ }^{4}$ Hamilton, ON

BACKGROUND: Evidence regarding the risk of influenza infection associated with healthcare is sparse.

PURPOSE: To assess risk factors for pandemic influenza infection in healthcare workers.

METHODS: From enrollment to 31/3/2010, acute care hospital healthcare workers $(\mathrm{HCW})$, other working adults and their households were followed weekly for symptoms of and risk factors for acute respiratory illness (ARI). Midturbinate swabs for influenza PCR were submitted for each ARI.

RESULTS: From 1143 adults and 218 children enrolled in the study, there were 818 episodes of ARI, of which $73(8.9 \%)$ yielded pH1N1 and $1(0.1 \%) \mathrm{H} 3 \mathrm{~N} 2$. Children were more likely to develop symptomatic influenza (SFlu) than adults (21.6\% (47/218) vs. 2.4\% (27/1143), $\mathrm{p}<0.001)$. Among adults, 2.0\% (14/685) HCW and 2.8\% (13/458) non-HCW developed SFlu $(\mathrm{p}=0.39)$. HCW were more likely to be vaccinated against $\mathrm{pH} 1 \mathrm{~N} 1$ than non-HCW (407/685 (59.4\%) vs. 135/485 $(29.5 \%), \mathrm{p}<0.001)$. Adjustment for vaccination did not reveal a higher risk of SFlu in HCW (odds ratio (OR) (95\% confidence interval (CI)): $1.16(0.53,2.53)$. Multivariable analysis showed enhanced risk of SFlu 
in subjects sharing households with children (OR (95\% CI): 7.0 (2.9, 16.8)) and participants with allergies (OR (95\% CI): $2.5(1.0,6.3))$. Receipt of $\mathrm{pH} 1 \mathrm{~N} 1$ vaccine was protective (OR (95\% CI): 0.08 (0.02, 0.36)). Among $\mathrm{HCW}$, those spending time in rooms where aerosol generating medical procedures (AGMP) were performed were more likely to develop SFlu (9/166 (5.1\%) vs. 5/478 (1.0\%), p=0.0031), but not those performing or assisting AGMP (4/145 (2.8\%) vs. 10/533 $(1.9 \%), \mathrm{p}=0.51)$ or those with routine contact with patients with ARI $(10 / 332(3.0 \%)$ vs. $4 / 347(1.2 \%), p=0.09)$.

CONCLUSIONS: Our data contradict the hypothesis that HCW status is associated with a higher risk for influenza. Household contact with children was the major risk factor for $\mathrm{pH} 1 \mathrm{~N} 1$ in our cohort. Among HCWs, exposure to rooms during AGMP may pose a risk.

\section{P136}

\section{VACCINATION CONTRE LA GRIPPE A(H1N1) CHEZ LES} ENFANTS FRÉQUENTANT UN CENTRE DE LA PETITE ENFANCE EN MONTÉRÉGIE

J Gagné ${ }^{1}$, F Leduc ${ }^{1}$, J Lussier ${ }^{1}$, C Messier ${ }^{1}$, R Pressé ${ }^{1}$,

G Baron ${ }^{1,2,3}$, J St-Cerny ${ }^{1,2,3}$

${ }^{1}$ Sherbrooke, QC; ${ }^{2}$ Longueuil, QC; ${ }^{3}$ Greenfield Park, QC

CONTEXTE : En 2009, une campagne de vaccination de masse a été entreprise afin de contrer l'épidémie de grippe A(H1N1). Les centres de la petite enfance (CPE) constituent des milieux propices à la propagation virale et regroupent l'un des groupes prioritaires (enfants 6 mois à 5 ans) ciblés pour la vaccination contre cette grippe.

OBJECTIFS : 1 . Évaluer la couverture vaccinale (CV) contre la grippe $\mathrm{A}(\mathrm{H} 1 \mathrm{~N} 1)$ chez les enfants fréquentant un CPE en Montérégie et identifier les déterminants et motifs ayant influencé cette vaccination.

2. Décrire les sources d'information utilisées par les parents et évaluer leur appréciation du déroulement de la campagne de vaccination.

MÉTHODE : Enquête par questionnaire auto-administré auprès des parents de 943 enfants inscrits dans l'un des neuf CPE de la Montérégie s'étant portés volontaires pour l'étude.

RÉSULTATS : Le taux de réponse obtenu était de 30,2\%. La CV a atteint $89 \%$ chez les enfants des CPE. La peur des conséquences de la grippe était le motif de vaccination le plus souvent cité alors que le manque de confiance dans le vaccin prédominait comme motif de nonvaccination. Le statut vaccinal contre la grippe $\mathrm{A}(\mathrm{H} 1 \mathrm{~N} 1)$ (du parent) et contre l'influenza saisonnière (de l'enfant), la perception d'une susceptibilité et d'une gravité accrues pour la grippe A (H1N1), de l'innocuité et l'efficacité du vaccin ont été des déterminants en faveur de la vaccination. Les principales sources d'information utilisées par les parents étaient les reportages télévisés et les médecins/autres professionnels de la santé. Enfin, les répondants se sont dits généralement en accord avec le déroulement de la campagne de vaccination.

CONCLUSION : Lors d'une future campagne de vaccination contre une souche pandémique de la grippe, promouvoir la vaccination en ciblant la susceptibilité individuelle et la gravité de la maladie tout en rassurant la population sur l'innocuité du vaccin utilisé.

\section{P137}

THE POWER OF COLLECTING INDIVIDUAL LEVEL VACCINE DATA USING AN IMMUNIZATION INFORMATION SYSTEM DURING PANDEMIC H1N1 INFLUENZA

\section{JM Nassif, A Gentry, J Biletchi}

Thorold, ON

BACKGROUND: The Protocol for Electronic Clinic Systems software is an immunization information system that automates client immunization records, efficiently processes clients, and provides a central database for analysis and reporting. Niagara Region Public Health used this software to support mass immunization clinics during pandemic (p) H1N1.

PURPOSE: To promote the benefits of collecting individual vaccine data using an immunization information system.
METHODS: Data were analyzed using SQL Reporting Services (2005) and presented in Microsoft Excel format. Statistics, including client counts, and mean average processing time for client (with reception and nurse), were calculated to demonstrate effects on clinic operational efficiencies. These included the effects on average processing times of increased client numbers, policy changes around vaccine eligibility, and whether clients attended the clinic as a family. Geographic Information Systems were used to analyze the distribution and uptake of $\mathrm{pH} 1 \mathrm{~N} 1$ vaccine throughout Niagara.

RESULTS: In Niagara, a total of 54,288 clients received the pH1N1 vaccine at clinics. The fastest processing times were $0: 40$ minutes with registration and 2:26 minutes with a nurse. These occurred when there was steady client demand without any restriction on vaccine eligibility. Software data provided the ability to assess specific real-time and daily trends in clinic operations, evaluate average registration and nursing time per client, and manage reductions in processing times. In addition, as the number of clients increased, the average processing time for both registration and nursing times decreased. However, the more restrictions placed on client eligibility, the longer the average processing time per client.

CONCLUSION: By collecting individual data, a valuable and immediate review of various factors influencing clinic operations and geospatial analysis on vaccine coverage and surveillance can occur in real-time. These accurate and comprehensive studies will guide future planning and policy decisions.

\section{P138}

\section{TIME AND MOTION STUDY TO COMPARE ELECTRONIC AND HYBRID DATA COLLECTION SYSTEMS DURING THE PANDEMIC (H1N1) 2009 INFLUENZA VACCINATION CAMPAIGN}

$\underline{\text { S Quach }}{ }^{1}$, J Pereira ${ }^{1}$, J Hamid ${ }^{1}$, C Heidebrecht ${ }^{1}$, J Foisy ${ }^{1}$, S Quan $^{1}$, J Bettinger ${ }^{2}$, D Buckeridge ${ }^{3}$, S Deeks ${ }^{1}$, M Finkelstein ${ }^{1}$, CA Sikora ${ }^{4}$, JC Kwong ${ }^{1}$

${ }^{1}$ Toronto, ON; ${ }^{2}$ Vancouver, BC; ${ }^{3}$ Montreal, QC; ${ }^{4}$ Edmonton, AB BACKGROUND: Electronic and hybrid (combining paper-based and electronic methods) data collection systems were used to collect patient-level H1N1 immunization data during the vaccination campaign.

OBJECTIVES: To measure the time spent by clinic staff on data collection and compare efficiencies between electronic and hybrid systems.

METHODS: Between October 28th and December 18th, 2009, we observed a sample of 79 immunization clinics within 38 organizations across 9 provinces and territories. We measured time spent by staff on tasks related to data collection: registration, medical history collection, vaccine record keeping, preparation of documentation of vaccination and post-vaccination data entry. For each task, we compared median and mean times spent by method (paper vs. electronic). Total data collection mean time was assessed across systems (hybrid vs. electronic). RESULTS: The total mean time spent per client on data collection was 104 seconds using an electronic system; 143 seconds using a hybrid system with electronic client registration; and 172 seconds using a hybrid system with paper client registration. Across the systems, most data collection time was spent on client registration (mean time of 50 seconds per client), whereas medical history collection took 34 seconds per client, and record keeping took 27 seconds per client. Not all organizations provided documentation of vaccine or entered data into an electronic database after the immunization process; however, when these were measured they took a median of 13 seconds and 40 seconds per client, respectively. Electronic client registration and record keeping had significantly shorter data collection times than paper-based methods $(\mathrm{p}<0.001)$.

CONCLUSION: Overall, electronic systems were observed to be more time efficient than hybrid systems for collecting influenza 
immunization data. Electronic client registration and record keeping provided the greatest reduction in overall data collection times.

\section{P139 \\ ANAPHYLAXIS AND ALLERGIC-LIKE REACTIONS FOLLOWING ADMINISTRATION OF AS03-ADJUVANTED PANDEMIC H1N1 VACCINE: SURVEILLANCE SUMMARY FROM QUEBEC, CANADA \\ I Rouleau ${ }^{1}$, G De Serres ${ }^{1}$, M Benoît ${ }^{1}$, E Toth ${ }^{1}$, S Ménard ${ }^{1}$, M Landry $^{1}$, J Grenier ${ }^{1}$, G Trudeau ${ }^{1}$, M Tremblay ${ }^{1}$, R Roussel $^{1}$, S Giroux ${ }^{1}$, DM Skowronski² \\ ${ }^{1}$ Quebec, QC; ${ }^{2}$ Vancouver, BC}

BACKGROUND: In Quebec, 4.8 million people received a monovalent AS03-adjuvanted vaccine domestically produced (Arepanrix ${ }^{\mathrm{TM}}$; GlaxoSmithKline). We report the provincial rate of anaphylaxis and allergic-like reactions per 100,000 doses administered and describe associated characteristics.

MATERIALS AND METHODS: In Quebec, reporting adverse events following immunization (AEFI) is mandatory for physicians and nurses. Cases of anaphylaxis and allergic-like reactions reported during the campaign were reviewed and analyzed.

RESULTS: Allergic-like reactions were reported at a rate of 11 per 100,000 doses (521 patients) compared to a historic average of 4 per 100,000 (2003-2008). Anaphylaxis was specified for 34 patients $(0.57$ per 100000 doses) but 39 others received adrenalin after reactions involving 1 (13 patients) or 2 systems (26 patients). Onset of symptoms was within 30 minutes for $81 \%$ of patients reporting anaphylaxis and $72 \%$ among those otherwise receiving adrenalin. For patients reporting any allergiclike reaction, mean delay between vaccination and symptom onset was 15 hours and mean symptom duration was 3.3 days. Women comprised $75 \%$ of patients reporting allergic-like reactions. Relative risks for immunized women (compared to men) was substantially greater during reproductive years, peaking at 8.4 (CI 95\% 3.7-18.9) for women 30 39 years old, but averaged 1.0 for cases under 10 or over 60 years of age. No patients reported a preexisting vaccine allergy, but $59 \%$ of patients reported hypersensitivity to at least one allergen (inhalant, drug, food, etc). Urticaria was reported by $49 \%$ of cases, angioedema by $31 \%$ and dyspnea by $16 \%$.

CONCLUSION: Compared to previous seasonal influenza vaccine experience, a higher rate of anaphylaxis and allergic-like reactions was detected by the Quebec surveillance system per doses of ASO3. adjuvanted $\mathrm{pH} 1 \mathrm{~N} 1$ vaccine administered in 2009-10. These reports came disproportionately from immunized women compared to men, especially women of reproductive age. Further investigation of the cause and risk factors is proposed.

\section{P140}

IS A MASS IMMUNIZATION PROGRAM FOR PANDEMIC (H1N1) 2009 GOOD VALUE FOR MONEY? EVIDENCE FROM THE CANADIAN EXPERIENCE

B Sander ${ }^{1}$, CT Bauch ${ }^{2}$, D Fisman ${ }^{1}$, RA Fowler ${ }^{1}$, JC Kwong1, $\overline{\text { A Maetzel }}^{3}$, A McGeer ${ }^{1}$, J Raboud ${ }^{1}$, D Scales ${ }^{1}$, M Zivkovic Gojovic ${ }^{1}$, MD Krahn ${ }^{1}$

${ }^{1}$ Toronto, ON; ${ }^{2}$ Guelph, ON; ${ }^{3}$ Basel, Switzerland

BACKGROUND: In response to pandemic H1N1 influenza 2009 outbreak, many jurisdictions undertook mass immunization programs. The objective of this study was to determine the cost-effectiveness of the mass H1N1 immunization program in Ontario, Canada's most populous province (population 13,000,000).

METHODS: A cost-utility analysis comparing the H1N1 mass immunization program in Ontario to no intervention was performed from the health care payer perspective. The economic evaluation is informed by a simulation model of a pandemic H1N1 2009 outbreak in a city in Ontario. Health outcomes measured included number of cases, number of deaths and QALYs. Probabilities for health care resource use (office visits, emergency department [ED] visits, hospitalizations) and deaths were based on Ontario pandemic H1N1 surveillance data and administrative data. Costs included immunization program cost and health care cost for treating H1N1 cases and were drawn from Ontario administrative data sources. Primary outcomes were quality adjusted life-years (QALYs), costs in 2009 Canadian dollars, and cost per QALY gained (incremental cost-effectiveness ratio [ICER]).

RESULTS: We estimated that 4.1 million cases of symptomatic influenza would have occurred (31.5\% symptomatic attack rate) in the absence of an immunization program. Our model predicted that $22 \%$ of symptomatic cases, $22 \%$ of office and ED visits, $23 \%$ of hospitalizations, and of $25 \%$ of death were prevented by the program. While the program was costly (C $\$ 180,400,000)$, it was also highly cost effective at $\mathrm{C} \$ 8,726 / \mathrm{QALY}$ gained. Projections were most sensitive to the timing of the immunization program and less sensitive to immunization program cost and QALYs. In all deterministic sensitivity analyses the ICER remained well below WHO thresholds for cost-effectiveness. Finally, probabilistic sensitivity analysis showed the H1N1 mass immunization program in Ontario to be cost-effective in all simulations $(100 \%)$ at a willingness-to-pay of $\$ 25,000$ per QALY.

CONCLUSIONS: This analysis suggests that a mass immunization program as carried out in Ontario in response to H1N1 2009 was not only effective in preventing influenza cases and health care resource use but was also highly cost-effective despite the substantial program cost.

\section{P141}

PCIRN STUDY OF ADULTS GIVEN MONOVALENT, ADJUVANTED H1N1 VACCINE WITH CONCURRENT OR DELAYED TRIVALENT SEASONAL INFLUENZA VACCINATION

DW Scheifele $^{1}$, M Dionne ${ }^{2,3}$, G De Serres ${ }^{2,3}$, M Loeb $^{3,4}$, A McGeer $^{3,5}$, O Vanderkooi ${ }^{3,6}$, J Kellner ${ }^{3,6}$, S Dobson ${ }^{1,3}$, L Sauve $^{1,3}$, Y Li $^{3,7}$, B Law ${ }^{3,8}, \mathrm{~S} \mathrm{Halperin}^{3}$ ${ }^{1}$ Vancouver, BC; ${ }^{2}$ Quebec City, QC; ${ }^{3}$ Halifax, NS; ${ }^{4}$ Hamilton, ON; ${ }^{5}$ Toronto, ON; ${ }^{6}$ Calgary, AB; ${ }^{7}$ Winnipeg, MB; ${ }^{8}$ Ottawa, ON

BACKGROUND: When adjuvanted $\mathrm{pH} 1 \mathrm{N1}$ vaccine was recommended for Canadians in 2009, compatibility with concurrently administered trivalent inactivated seasonal vaccine (TIV) had not been assessed.

PURPOSE: To compare responses of adults given pH1N1 and TIV vaccines, either concurrently (in opposite limbs) or sequentially (TIV delayed by 21 days).

METHODS: Five PCIRN centers enrolled adults 20-59 years old who were randomly assigned (1:1) to receive adjuvanted, monovalent H1N1 vaccine (Arepanrix ${ }^{\circledR}$, GSK Laval) with either concurrent (Group C) or delayed (Group D) TIV (Fluviral®, GSK Laval) vaccination. All subjects were bled at study entry and 21 days after each vaccination. Serum antibodies to vaccine components were measured by hemagglutination inhibition (HAI) assay and analyzed by standard criteria.

RESULTS: 290 subjects were enrolled (C:146, D:144) and 281 completed the study (C:145, D:136). Groups were similar in age (mean 40.0 years), sex distribution (60\% female), BMI (mean 25.8 ), racial distribution ( $>80 \%$ white) and health status. Pre-existing antibody to pH1N1 was detectable (titer $\geq 10$ ) in $34 \%-36 \%$; titers were $\geq 40$ ("protective") in $15 \%-18 \%$ at baseline and in $91 \%-92 \%$ post-vaccination. Post-immunization titers $\geq 40$ were present in $96 \%-100 \%$ and $86 \%$ $89 \%$ of subjects with and without pre-existing antibody, respectively. Post-immunization geometric mean titers (GMT) were 3-fold higher in those with pre-existing pH1N1 antibody. Responses to H3N2 did not differ between groups. For seasonal H1N1 antigen, Group D subjects had lower final GMTs (p 0.003). For B/Brisbane, Group D had lower responses than Group $\mathrm{C}$ by most measures (e.g. final titers $\geq 40$ in $58 \%$ vs $74 \%$ (p 0.006) and final GMT 44 vs 64 (p 0.017), respectively), despite equivalence of baseline measurements. 
CONCLUSIONS: Responses to $\mathrm{pH} 1 \mathrm{~N} 1$ vaccine were robust and unaffected by concurrent TIV administration. Responses to H3N2 vaccine were equivalent between groups whereas responses to B/Brisbane differed significantly between groups. The basis for this difference is unclear but it favored concurrent vaccinations.

\section{P142 \\ PATIENT FLOW IN MASS VACCINATION CLINICS: IDENTIFYING THE AMOUNT OF TIME PATIENTS STAY IN KEY AREAS OF THE 2009/10 pH1N1 CLINICS IN ALBERTA, CANADA}

\section{Sikora, B McKim, M Johnson, The PCIRN Investigators \\ Edmonton, $\mathrm{AB}$}

BACKGROUND: A province-wide influenza immunization campaign was made available to the approximately 3.5 million inhabitants of Alberta, Canada in the winter of 2009. It is difficult to match the clinic processes of registration, immunization and observation to the demands of the arriving patients. Measuring the time spent in each of these key areas is vital to understanding patient flow through clinics. This information can be used to better structure vaccination clinics and to improve immunization delivery to populations.

PURPOSE: To describe the amount of time individual patients spent in key areas of vaccination clinics in Alberta during the 2009/10 pH1N1 season.

METHODS: A time \& motion study was completed at selected pH1N1 mass vaccination clinics in Alberta. A random sample of patients arriving at clinics was chosen at each site. Each patient was followed and timed as they progressed through the clinic. The time periods recorded included: entry into the line-up to the completion of registration, completion of registration to arrival for immunization, arrival to departure from the immunization station, transport from the immunization station to entry into the observation area, and entry to exit from the observation area.

RESULTS: There were a total of 442 individual patients observed at eight (50\% urban) clinic sites between December 7 and 18, 2009. There were 358 patients that completed the registration, immunization and observation stages. The average time spent in the clinic was 35.9 minutes (95\% CI=34.4-37.6). On average, patients stayed 9.5 minutes in registration $(95 \% \mathrm{CI}=8.2-10.9), 9$ minutes at the immunization station ( $95 \% \mathrm{CI}=8.5-9.6)$, and 12 minutes in the observation area $(95 \% \mathrm{CI}=11.5-12.6)$. Rural clinics were faster than urban clinics for registration $(4.9$ vs. 10.9 minutes, $\mathrm{p}<0.05)$ and immunization $(7.8$ vs. 9.4 minutes, $\mathrm{p}<0.05)$.

CONCLUSIONS: Roughly $50 \%$ of patient time at mass vaccination clinics is spent interacting with clinic staff. Improving clinic processes, to decrease the face-to-face time, can potentially improve staff safety. Novel approaches to registration, consent, immunization and data recording may be able to decrease the staff-contact time and ultimately increase the throughput of mass vaccination clinics.

\section{P143}

\section{A SURVEY OF PERCEPTIONS OF SAFETY, PRIVACY AND DATA SECURITY OF PATIENTS ATTENDING PH1N1 MASS VACCINATION CLINICS IN ALBERTA, CANADA}

\section{Sikora, B McKim, M Johnson, The PCIRN Investigators}

\section{Edmonton, $\mathrm{AB}$}

BACKGROUND: A province-wide influenza immunization campaign was made available to the approximately 3.5 million inhabitants of Alberta, Canada in the winter of 2009. The delivery of the pH1N1 vaccine was primarily through temporary mass vaccination clinics. During times of emerging health threats, leaders are challenged with maintaining security, safety and privacy within such clinics.

PURPOSE: To identify the perceptions, on safety, privacy and data security, of patients who attended $\mathrm{pH} 1 \mathrm{~N} 1$ mass vaccination clinics.
METHODS: A convenience sample of clinic sites was chosen for administration of a patient survey between December 7 and 18, 2009. At each clinic site, patients were invited to complete the survey, following vaccination while waiting in the observation area of the clinic. The anonymous survey identified patient agreements to the statements: 'My privacy was maintained at the immunization clinic', 'I felt safe during my time at the immunization clinic' and 'I felt my data security (personal information) was protected in the immunization clinic'.

RESULTS: There were 8 sites that distributed the survey with 1601 returned. Approximately $90.9 \%$ of respondents agreed that their privacy was maintained at the clinic (1.3\% disagree). Of all respondents, $96.1 \%$ agreed that they felt safe at the clinic $(0.6 \%$ disagreed $)$ and $87.4 \%$ agreed that their data security (personal information) was protected at the immunization clinic ( $1.5 \%$ disagreed). No differences were identified between the responses by geographic region, gender or age group.

CONCLUSIONS: A very small proportion of patients felt unsafe even though many clinics were temporary in nature. Maintenance of privacy and data security were less supported in the patients surveyed, suggesting a need to enhance these aspects of clinic delivery.

\section{P144}

\section{A SURVEY OF INFORMATION SOURCES UTILIZED BY PATIENTS ATTENDING $\mathrm{pH} 1 \mathrm{~N} 1$ MASS VACCINATION CLINICS IN ALBERTA, CANADA}

\section{Sikora, B McKim, M Johnson, The PCIRN Investigators}

Edmonton, AB

BACKGROUND: A province-wide influenza immunization campaign was made available to the approximately 3.5 million inhabitants of Alberta, Canada in the winter of 2009. Health planners are challenged with promoting vaccines to susceptible populations. To increase vaccine uptake in populations, it is important to identify the factors influencing the choice to be immunized and the information sources used to make this decision.

PURPOSE: To identify the factors influencing patients' choice to be immunized and to determine the sources of information used for this decision.

METHODS: A convenience sample of clinic sites was chosen for administration of a patient survey between December 7 and 18, 2009. At each clinic site, patients were invited to complete the survey, following vaccination while waiting in the observation area of the clinic. The anonymous survey identified patient viewpoints regarding factors influencing their decision to receive the H1N1 vaccine, and their sources of information about the H1N1 vaccine. Participants could choose multiple responses to these questions to reflect the multiple sources patients use for information. Categories for these responses were pre-identified by consensus by the investigators.

RESULTS: There were 8 sites that distributed the survey with 1601 returned. Approximately $52 \%$ of patients surveyed reported that the media/internet influenced their decision to be immunized. Other factors influencing patient's decision to immunize were: recommendation from physician or health care professional $(41 \%)$, recommendation from friends (22\%) and previous experience with influenza (18\%). The information sources used by patients attending the clinics were the news media (73\%), and friends/family (38\%). A smaller percentage of patients used information from the internet (29\%), family physician $(22 \%)$ and their pharmacist (2\%).

CONCLUSIONS: This study identified the media and health professionals as major influencers of patients' decisions to immunize, and news media, family and the internet as the most common sources of vaccine information. Vaccine planning organizations should involve health professionals in their program strategy and should focus on using media to disseminate information. 
P145

\section{STAFF PERCEPTIONS OF EFFICIENCY OF VACCINE DELIVERY IN THE 2009/10 pH1N1 MASS VACCINATION CAMPAIGN IN ALBERTA, CANADA}

\section{Sikora, B McKim, M Johnson, The PCIRN Investigators \\ Edmonton, AB}

BACKGROUND: A province-wide influenza immunization campaign was made available to the approximately 3.5 million inhabitants of Alberta, Canada in the winter of 2009. The campaign was organized and delivered by a single health administration system, using staff from existing and seconded positions. The opinions of these staff can be used to improve the efficiency of future mass vaccination clinics.

PURPOSE: To identify staff perceptions of the barriers for efficient delivery of vaccine at mass vaccinations clinics during $\mathrm{pH} 1 \mathrm{~N} 1$ and exploring areas for future improvement.

METHODS: Semi-structured focus groups were held after completion of the 2009/10 pH1N1 influenza vaccination campaign. Staff who worked in the positions of front line worker, manager, Communicable Disease Control nurse and Medical Officer of Health were invited to participate. The researchers facilitated each worker category separately. The focus groups were recorded, transcribed and coded for emerging themes using a grounded theory approach.

RESULTS: There were 17 focus groups facilitated involving 198 participants (median of 11 participants/group). Staff were represented from all urban and rural delivery areas in Alberta. Major themes of barriers to efficiency included: a prescriptive approach to clinic design, communication breakdown within the organization, mismatch between client demand and staffing, bottlenecks in clinic processes (registration, consent, and immunization) and data management factors. Areas to improve clinic efficiency included: building on the expertise of staff members, allowing flexibility to change to the local delivery environment and developing novel mechanisms to address bottlenecks in registration, consent and data management.

CONCLUSION: These focus groups identified, from a staff perspective, major efficiency barriers of the 2009/10 pH1N1 campaign. It also identified areas for improvement that would be acceptable to workers at future mass vaccination clinics. Clinic organizers, planners and funders can use this information to better deliver services to the population.

\section{P146 \\ IMMUNIZATION TRAINING: PREPARING PHARMACISTS FOR AN EXPANDED SCOPE OF PRACTICE}

\section{J Isenor, C Tobin, S Bowles, K Slayter \\ Halifax, NS}

BACKGROUND: Immunization rates for vaccine preventable diseases are not at optimal levels for many patient populations in Canada. Among the strategies suggested to improve these rates is the training of non-traditional immunization providers to administer vaccines safely and effectively in their practice settings. Regardless of the provider, it is important to have a comprehensive knowledge of immunization.

PURPOSE: We report on a competency based program, the Dalhousie University Continuing Pharmacy Education Immunization and Injection Administration Training Program (IIATP) that was utilized to train pharmacists to become immunizers.

METHODS: A 12 hour online competency based immunization program previously developed by the British Columbia Centre for Disease Control and a full day certification workshop developed by the British Columbia Pharmacists Association and the Alberta Pharmacists Association were modified to meet the needs and requirements of pharmacists within our catchment. Enrollment in IIATP began in the fall of 2009. Participants were asked to complete a brief online survey after completion of the program that included demographic data and immunization activities.
RESULTS: Of the 231 pharmacists who have enrolled in the program, 228 have successfully completed all components. Eighty-seven pharmacists completed the survey. Most respondents (91.9\%) practice in community pharmacy. Over 23\% have been authorized to inject medications in their province (New Brunswick). The others are awaiting legislation in their respective provinces. Of the 20 pharmacists authorized to inject, 11 have administered a medication by injection (including vaccines). Over 63\% (52) of respondents have reported being actively solicited by their patients on a daily to monthly basis to administer immunizations. Nearly $83 \%$ of respondents agree or strongly agree that they feel prepared to administer immunizations.

CONCLUSION: Our results show that pharmacists who have completed IIATP are ready to expand their scope of practice to include administration of immunizations against vaccine preventable diseases.

\section{P147}

\section{ADHERENCE TO SEQUENCING FOR PANDEMIC VACCINE IN SK DURING THE 2009 PANDEMIC MASS VACCINATION CAMPAIGN}

\section{B Adhikari, R Tuchscherer, S Shahab \\ Regina, SK}

BACKGROUND: The Canadian Influenza Pandemic Plan recognises that the entire population can not be vaccinated at the same time during a pandemic due to a variety of factors including vaccine supply and operational capacity. It has provided a framework for recognising a variety of priority groups based on risk factors for serious illness (age and underlying health conditions) as well as occupational groups identified to be either at high risk of exposure (health care workers) or part of essential services (fire, police, utilities etc).

PURPOSE: During the 2009 H1N1 Pandemic, a nationally agreed upon framework for sequencing was developed based on expected rate of vaccine supply, operational capacity to deliver the vaccine, and epidemiology of the pandemic in terms of risk factors for serious illness. The exact details of the groups varied from province to province. This study presents an analysis of adherence of the population to the sequenced groups announced in Saskatchewan, Canada.

METHODS: The Saskatchewan Immunization Management System was used to assess percentage uptake by sequenced groups after announcement. It was clearly communicated that once a group became eligible they remained eligible in an ongoing manner.

RESULTS: Uptake for each sequenced group was highest in the fourteen days after that particular group became eligible for vaccination. CONCLUSION: Defining and clearly communicating sequenced groups is a feasible way to manage mass pandemic vaccination clinics based on rate of vaccine supply and capacity to deliver the vaccine. The public is able to understand and adhere to the sequencing.

\section{P148}

\section{ANAPHYLAXIS FOLLOWING ADMINISTRATION OF H1N1 VACCINE IN CANADA: CASE VALIDATION AND RAPID CLINIC ASSESSMENT TOOLS}

\author{
J Tustin, R Pless, B Law, H Zheng \\ Ottawa, ON
}

BACKGROUND: The H1N1 influenza pandemic resulted in the largest mass immunization campaign in recent Canadian history. Vaccine safety surveillance was enhanced as the vaccine was a newly formulated adjuvanted one not previously used in Canada. After the first two weeks of the campaign, a higher than expected number of cases of anaphylaxis were reported. Validating them proved challenging and the reliability of a standardized case definition had not been tested.

PURPOSE: The objective was to determine how well public health professionals applied a case definition scoring tool for anaphylaxis, including assessing inter-reviewer variability with case assessments, in order to help develop more robust case classification tools. 
METHODS: Eighteen independent expert reviewers used a newly developed scoring tool based on the "Brighton" case definition of anaphylaxis to score and classify on level of diagnostic certainty, twentythree reported cases of anaphylaxis.

RESULTS: Based on the most agreed level of classification, the results showed that many cases reported initially as anaphylaxis did not meet the case definition with a $56.5 \%$ difference. Substantial inter-reviewer variability was also evident in the identification of signs/symptoms and in the subsequent classification as to level of diagnostic certainty; only three cases $(13 \%)$ had more than $80 \%$ agreement on the level of diagnostic certainty. The percent of overall agreement was 0.54 with a kappa statistic of $0.4(\mathrm{p}<0.001)$.

CONCLUSION: The inter-reviewer variability shows a need for education and training for health professionals both reporting and classifying cases of anaphylaxis. Information provided on case reports may be inadequate to allow for proper classification according to strict case definitions such as those developed by the Brighton Collaboration. Therefore, the specificity of the case scoring form may not be suitable for provincial or federal case classification, but with adequate training, more applicable at the local level where cases initially present.

\section{P149}

\section{TRACEBACK INVESTIGATION OF H1N1 VACCINE SHOEBOX} LOT 7A IN MANITOBA AND SASKATCHEWAN

\section{J Tustin ${ }^{1}$, R Pless ${ }^{1}$, B Law ${ }^{1}$, R Tuchscherer ${ }^{2}$, M Long ${ }^{3}$}

${ }^{1}$ Ottawa, ON; ${ }^{2}$ Regina, SK; ${ }^{3}$ Winnipeg, MB

BACKGROUND: Following the second week of mass distribution of the H1N1 vaccine, a higher than expected number of anaphylaxis reports was detected via enhanced surveillance to the Public Health Agency of Canada. Several reports were linked to Shoebox Lot 7A of the vaccine. As a precaution, lot 7A was withdrawn from the immunization campaign.

PURPOSE: The objectives were to determine the movement of lot 7A from the manufacturer to immunization clinics, and to determine jurisdiction-specific practices in vaccine delivery in order to identify any potential causes for the higher than expected number of anaphylaxis reports. This was done in combination with retrieval of vaccine lots for testing by Health Canada and the manufacturer, as part of a comprehensive "root-cause" analysis of this event.

METHODS: A semi-structured questionnaire was administered in person or by telephone to key informants from the regional health authorities and First Nations health jurisdictions of the two most affected provinces. The questionnaire focussed on the movement of lot 7A, cold chain excursions, and vaccine shipment, storage, distribution and delivery practices.

RESULTS: Vaccine shipment, storage and distribution practices did not vary considerably across regions or provinces and adhered to acceptable protocols. In contrast, vaccine administration practices varied in terms of warming times, reconstitution and injection methods and immunizer training/experience. Valuable feedback was received from front-line staff on their experience in the local and national delivery of the H1N1 vaccine.

CONCLUSION: Expert consultation and epidemiologic studies are needed to further explore and understand the potential effect of H1N1 vaccine administration practices on the presence or severity of adverse reactions. This was the first investigation of this nature in Canada. The lessons learned will aid in the development of adverse events following immunization signal response protocols, in order to improve the timeliness, coordination and standardization of future responses and investigations.
P150

\section{9 pH1N1 INFLUENZA MASS IMMUNIZATION CLINICS: EFFECTIVENESS IN AN ISOLATED FIRST NATION COMMUNITY}

A Bennett ${ }^{1}$, A Abdi $^{2}$

${ }^{1}$ Kashechewan, ON; ${ }^{2}$ Mississauga, ON

BACKGROUND: During the pH1N1 Influenza Pandemic each community faced its own challenges. Kashechewan, Ontario is a remote First Nation community on the James Bay Coast. It has a population of approximately 1600 of whom, approximately 1000 are under 25 years of age. There is a very high incidence of chronic disease.

Health Canada's Nursing Station is the only health care service available within the community. The Nursing Station is staffed by six Primary Care Nurses, one Nurse-In-Charge, and two Public Health Nurses.

PURPOSE: The isolation of the community, together with the demographics, posed a high risk. Vaccinations needed to be carried out in a timely and efficient manner in order to prevent an outbreak of pH1N1 Influenza. The Pandemic Planning committee decided to implement Mass Immunization Clinics (MIC's).

METHODS: The school gym was chosen as a centrally located venue capable of accommodating large numbers of people. Four immunization stations were established. The space was setup to provide an orderly flow of patients from the time they entered until they exited. At each station, a nurse worked with a support staff member, while other nurses were mixing and drawing the vaccine. First Responders were present. Health information was distributed throughout the process. RESULTS: The MIC's worked effectively in this community. Three MIC's were held. On average the patient wait time was less than one hour. Supplies of vaccine and health information were appropriate for the size of the community. Overall, a vaccination rate of approximately $80 \%$ was achieved.

CONCLUSION: Use of the MIC Toolkit was effective in this community during the 2009 pH1N1 Influenza Pandemic.

Among the factors that contributed to the success of the MIC's were: the mobilization of community workers who supported the nursing contribution; complete support from community leadership; and good planning and implementation of the MIC's.

\section{P151}

\section{PANDEMIC H1N1 MASS IMMUNIZATION IN NEW} BRUNSWICK FIRST NATION COMMUNITIES

SR Landsburg1', E McQuade1 ${ }^{1}$ P Birney1 , L Nicholas ${ }^{1}$, J Caplin², N Robichaud $^{2}$

${ }^{1}$ Fredericton, NB; ${ }^{2}$ Eel River Bar, NB

BACKGROUND: As a result of the severe impact that was seen in Aboriginal people living on reserve in other parts of Canada, it was important to ensure a safe and equitable process for H1N1 immunization that identified Aboriginal people as a priority in the New Brunswick H1N1 mass immunization planning. Therefore it was critical to develop a model to provide such a mass immunization program.

PURPOSE: To develop provincial public health framework for H1N1 immunization for First Nation community members that supported on reserve community based clinics.

METHODS:

- Critical planning dialogue occurred during summer 2009 prior to the vaccine. This occurred through a series of meetings with the New Brunswick FN chiefs and their Health Directors as well as the Regional Health Authorities (RHA), the NB Department of Health (DOH) and First Nation Inuit Health (Atlantic).

- RHA Public Health immunization teams collaborated with each individual community to plan for the H1N1 mass immunization program.

- Ongoing dialogue occurred between the $\mathrm{DOH}$ and the RHAs to ensure consistent provincial communication and planning occurred. 
- Identified a First Nation representative from each of the RHA's

- Regional Medical Officers of Health (RMOH) and Regional Public Health Directors visited each community

RESULTS:

- Linkages established with the New Brunswick First Nation advisory group

- Coverage rates for community members ranged from $82-100 \%$

- Established partnerships between the RHA and FN community health staff

- Community clinics created a high level of community involvement, level of trust

- Created interest from public health staff to learn more about the FN communities

CONCLUSION: The H1N1 mass immunization program for First Nations in New Brunswick was very successful. On average, there was $90 \%$ coverage from the community based clinics reflective of the creative planning by First Nations and with the Regional Health Authorities.

KEY FINDING: This model demonstrated community mass immunization program that was a success and supported the First Nations culture of community, family and friends. 
A

Abbas Z ................ P031, PO64

Abdi A ...................P150

Achonu C . . . . . . . . . . . . . . . . P085

Adhikari B .......... P052, P147

Aggarwal N . . . . . . . . . . . ......P133

Ahmadipour N ..P016, P099, P109, P130,

................. P131, P132

Al Khateeb HA ...............P021

Al Marzooqi AH ...............P021

Allan PA ............. P022, P027

Allen V . . . . . . . . . . . . . . . . P087

Allen WC ..................P110

Allison D ..................P082

Aly E .....................P122

Ambrose A ........ P101, P111, P127

Ambrozaitis A . ................P092

Andrews W ...................P053

Ann J . . . . . . . . . . . . . . . . . . . . . . P094

Anyoti H . . . . . . P016, P109, P131, P132

Aoki F ............... O13, O15

Appleton M ................. O11

Arnou R . . . . . . . . . . . . . . . . P092

Ashton A ...................P023

Ashton SG ................P110

Audet D ............. P025, P060

B

Babakissa C . . . . . . . . . . . . . . P002

Babiuk L .................... O13

Badger G . . . . . . . . . . . . . . . . . . .P043

Banerji A ...................P024

Bangura H . . . . . . . . . . . . . . . . P052

Banh L . . . . . . . . . . . . . . . . . . . . . .P098

Barnes S ....................P098

Baron G ..................P136

Barrowman N .................P100

Bartlett $\mathrm{G} \ldots \ldots \ldots \ldots \ldots \ldots . \ldots 04$

Bartlett M ....................P015

Bauch CT ............ P107, P140

Baxendale DM ..............P110

Beckermann K ....... O01, P079, P086

Bedell L . . . . . . . . . . . . . . . . . . . . . P008

Belzak L ....... . P032, P073, P075, P134

Benbernou A ..................P001

Bennett A . . ................P150

Benoit M . ........ P111, P127, P139

Bernard S . . . . . . . . . ........P002

Bettinger JA ...... O07, O10, O14, O18,

...........P034, P055, P065, P066, P067, P111, P112, P120,

................. P124, P128, P138

Biletchi J ..................P137

Biolchi A ....................P053

Birney P . . . . . . . . . . . . . . . P151

Bisson D .....................P024

Boccadifuoco G . . . . . . . . . . . . . . P053

Boivin G . . . . . . . . . . . P094, P101

Boliver DM .................P110

Bontavics E . . . . . . . P015, P059, P081
Boon $\mathrm{H}$

. P051

Booth T ............. P015, P055

Bortolussi B ........... O11, P056

Bortolussi R ................ O10

Bouchard S .................P113

Boucher F .......P014, P065, P066,

P067, P088, P120

Boulianne N .............P014, P025, P033, P034, P060, P062, .P065, P066, P067, P069,

$\ldots \ldots \ldots \ldots \ldots \ldots$ P088, P120, P121
Bowles S $\ldots \ldots \ldots \ldots$ P014, P088, P146

Bradet R .......... P034, P060, P066

Brisson M ............ P001, P061

Brown S . . . . . . . . . . . . . . . P012

Bryson M ....................P026

Buckeridge D . . . . . . . . . . . . . P138

Bujold E . . . . . . . . . . . . . . . . . . P087

Butler GA ........... P027, P082

Butt K ....................P082

\section{$\mathrm{C}$}

Camden S .................P001

Cameron J ........ O01, P044, P071, P114

Campbell D ...................P095

Campitelli MA ...............P083

Caplin J ....................P151

Carbon M ...................P003

Carruthers S . . . . . . . . . . . . . . P098

Chambers C ................ O11

Chambers LW .................P095

Chan J ...................... . O09

Chan S ......................P078

Cherkaoui S ................P104

Chokani K.................. O13

Chong-King E . . . . . . . . . . . P015

Chong M . . . . . . . . . . . . . . . . O 18

Chuck A .......... P096, P097, P103

Churko D .................P031

Clément P ......... P068, P069, P121

Coleman B ........P087, P111, P115,

................. P127, P135

Colledge E .................P038

Comanducci M ................P053

Connor D ...................P133

Cooper C . . . . . . . . . . . . . . . . . . . P116

Cooze HD ...................P045

Cote-Boileau T . . . . . . . . . . . P002

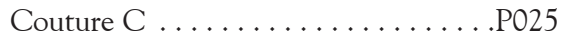

Couture M . . . . . . . . . . . . . . . P058

Crowcroft N ....... O06,O08, P081,

P085, P123, P128

Crowe $\mathrm{L} \ldots \ldots \ldots \ldots \ldots \ldots \ldots \ldots$. . . . . . . . . .

Cyr C ....................POO2

\section{$\mathrm{D}$}

D'Aoust M ..................P058

David S . . . . . . . . . . . . . . . O O17

Dawar M ...................P112

De Decker M ................P092
De Juanes Pardo J . . .............P092

De Serres G . . . . O O16, P060, P061, P062, .P111, P117, P127, P135, P139, P141

De Tora L . . . . . . . . . . . P007, P053

De Wals P .........P002, P003, P061, P062, P072, P117

Deceuninck G . . . P025, P060, P062, P117

Deeks SL ...... O06, P012, P063, P081, P085, P124, P128, P138

Deeter B ............ P028, P077

Defay F ........ P067, P120, P121

Demarinis C . . ...... O02, P029, P030

Deng S . . . . . . . . . . . . . . . P012

Denis M . . . . . . . . . . . . . . . P092

Dery P . . . . . . . . . . . . . . P055

Desai S .............. P015, P074

DesMeules M ............... O04

Diener T . . . . . . . . . . P031, P064

Diniz A ...................... 032

Dionne M ............. O16, P141

Dixon LM ....................P110

Do MT ............. P118, P119

Dobson S .............. O16, P141

Dodds L . . . . . . . . . . . . . . . . P102

Dolman S .....................P015

Donnelly J .............. P053, P054

Donovan T ................. O09

Doucette S ................P095

Douville Fradet M . . . . . . . . . . . . P003

Doyle C . . . . . . . . . . . . . . . . . P063

Drews $S \ldots \ldots \ldots \ldots \ldots \ldots \ldots$. . . . P087

Drolet M ............. P001, P061

Dubé E ... P014, P033, P034, P065, P066,

........ P067, P069, P088, P120, P121

Dubey V .......... O01, O11, P035,

P044, P071, P079

Dull P ... P004, P005, P006, P007, P008

$\mathrm{E}$

Earn D .................... O13

Earnshaw S. . . . . . . . . . . . . . . . P080

Eavis P . . . . . . . . . . . . . . P092

El Gak AI .................P021

Elliott D . . ..................P043

Embree J .....................P055

Esguerra L . . . . . . . . . . . . . . P023

Esposito S ........... P005, P006

$\mathrm{F}$

Fabry P $\ldots \ldots \ldots \ldots \ldots \ldots \ldots . . . . .2126$

FarhangMehr M ......... P043, P074

Farkouh R ...................P080

Fatoye B .................P036

Fediurek JK ..................P122

Fell D ........................P102

Finkelstein M ...... P015, P128, P138

Fisman D . ................P140

Foisy J .... P089, P123, P124, P128, P138

Fonseca K ........... O13, P106 
Fortin É .......................P003

Fowler RA ........... P125, P140

Freeland T . . . . . . . . . . . . . P047

Frescura A ......... P073, P075, P134

Fung $\mathrm{C} \ldots \ldots \ldots \ldots \ldots . \ldots 06$

\section{G}

Gabel B .......................P077

Gagné J ...................P136

Gagné L . . . . . . . . . . . . . . . O06

Gagneur A $\ldots \ldots \ldots \ldots \ldots$ P002, P126

Gardezi F . . . . . . ...........P035

Gariépy M ........... P111, P127

Garrahan T . . . . . . . . . . . .....P084

Geduld J . . . . . . . . . . . . . .PO26

Gemmill I .........P014, P065, P066,

P067, P088, P120

Gentry A .................P137

Gerges S ................P038

Gilca V ...........P014, P034, P065, P066, P067, P088, P120

Giles L . . . . . . . . . . . . . . . . . . P024

Gill CJ ....................P008

Gillespie M ................P023

Girgis A . . . . . . . . . . . P038, P040

Giroux S ................P139

Giuliani M ...............P053

Goodall EC . . . . . . . . . . . . . . . .P098

Gorringe S . . . . . . . . . . . . . . P028

Gouin K . . . . . . . . . . . . . . . P087

Gournis E ................P114

Granger M ............ P031, P064

Gray M ...................P090

Green KS ............ P110, P115

Greenaway C ............... O04

Greenberg S $\ldots \ldots \ldots \ldots \ldots \ldots$ P038

Grenier J . . . . . . . . . . . . . . .P139

Grenon R .................P100

Grimsrud K . . . . ....... P013, P056

Guay M ........P033, P060, P068, P069,

........ P089, P104, P121, P124, P128

Gubbay J ........006, P015, P020, P115

Guo Y ....................P087

Gupta A ............. P008, P133

Guttmann A ................P083

$\mathrm{H}$

Haase D . . . . . . . . . . . P017, P116

Halperin B . . . . . . . . P014, P034, P088

Halperin SA ..... O05, O07, O10, O11, O12, O14, O15, O16, .P008, P014, P055, P056, $\ldots \ldots \ldots \ldots \ldots$ P088, P101, P110, P115, Hamelin M ..................P094 Hamid A . . . . . . . . . . . . P068, P069 Hamid J ........................P138 Hammond $G$. . . . . . . . . . . . . . . O O15 Hanrahan A ................. O11 Harris T . ............... P043,P074 Hasselback P . . . . . . . . . . . . . P112 Heidebrecht CL. . P089, P125, P128, P138 Helferty M . . . . . P009, P010, P011, P037 Hemming $\mathrm{F} \quad \ldots \ldots \ldots \ldots . \ldots 02$
Henderson EA .......... P101, P105

Hendson L . . . . . . . . . . . . . . . . P087

Henning B . . . . . . . . . . P081, P085

Henteleff AC ................P129

Heys J ......................P084

Heywood D . . . . . . . . . . . . . . . . P129

Ho T ...................... 038

Hogan M .......... P038, P039, P040

Holcroft C . . . . . . . . . . . . . . . . . . O04

Horsman G ................. O13

Houle $S \ldots \ldots \ldots \ldots \ldots \ldots$........... 130

Hui C ...................PO24

Humber $\mathrm{L} \ldots \ldots \ldots \ldots \ldots \ldots \ldots$. . . . . . . . . . . . .

Hume A ....................P084

Hwang S .................P080

\section{I}

Icardi $\mathrm{G} \ldots \ldots \ldots \ldots \ldots \ldots$. . . P092

Inoue $\mathrm{M} \ldots \ldots \ldots \ldots \ldots \ldots \ldots . . . . . . . .2083$

Ipp M . . . . . . . . O O11, P038, P040

Isenor J . . . . . . . . . . . . . . P146

Ismail SJ ............ P043, P074

\section{$\mathrm{J}$}

Jacobs P ....... P070, P096, P097, P103

Jamieson F . . . . . . . . . . . . . P012

Jarvos $L \ldots \ldots \ldots \ldots \ldots \ldots \ldots 2$

Jeanfreau R ...................P008

Johnson D . . . . . . . . . . . . . . O05

Johnson ML ...............P042

Johnson R . ............ P001, P084

Johnson T P093, P142, P143, P144, P145

Johnston J .......... P087, P101, P135

Jordanov E................. O05

\section{$\mathrm{K}$}

Kaashoek J . . ............. P044, P071

Kadri O ..................... O01

Kallos A ....................P111

Karas E ....................P081

Karsten A . . . . . . . . . . . . . . P008

Katz KC ...................P087

Kayhty H . . . . . . . . . . . . . . . . . . . .P017

Keefe SC ....................P045

Keegan V . . . . . . . . . . . . . . P037

Kellner JD ........... O07, 016, P141

Khan A .....................P015

Khuc N ......................... 072

Kiberd M . . . . . . . . O12, P014, P088

Kiely M ............ P025, P121

Kikuta A . . ........... P035, P051

Kim S ............... P099, P130

Kimura A ..... P004, P005, P006, P007

Kirkwood M .................P105

Kitta A ......................P046

Klassen N .....................P043

Klein M ...................P116

Klein NP ..................P008

Kleinschmidt A . P004, P005, P007, P053

Knox-Kinsman, L . . . . . . . . . . . . . P084

Kobasa D . . . . . . . . . . . . . . . . P090

Kobinger GP ...............P090

Kolbe F.............. P114, P128

Krahn MD ............ P107, P140
Kris H. .....................P129

Kristjanson E ............... O06

Kucinska De Ocampo S . . . . . . . . . P023

Kurbis C....................P129

Kuster SP . . . . . . . . . . . . . . .P135

Kwan H . . . . . . . . . . . . . . . . . P080

Kwong JC...........P083, P089, P107, P124, P128, P138, P140

\section{$\mathrm{L}$}

Laddy D . . . . . . . . . . . . . . . . P090 Lafleche J ...........P016, P047, P099, P109, P131, P132

Lam P .....................P095

Lanctot K.....................P024

Landry M . . . . . . . . . . P069, P121, P139

Landry N . . . . . . . . . . . . . . . . . .P108

Landsburg SR ..............P151

Langley J. . . . O16, P043, P074, P110, P133

Laroche J ....... P043, P073, P075, P134

Lavoie F ........P014, P034, P065, P066, P067, P088, P120

Lavoie P . . . . . . . . . . . . . . P058

Law B ........ O07, O15, O16, P012, .P016, P047, P056, P099, P109, P115, P116, P130, P131, P132, P141, P148, P149

Law T .................. O14

Le Saux N .......... O10, P055, P100

Lebel M ......................P133

Leduc F . . . . . . . . . . . . . . . . P136

Lee B .............. P024, P087

Lee T ......................P017

Lefèbvre B ................... P062

Lemaire J . . . . . . . . . . . . P068, P069

Lemstra M . . . . . . . . . . . . . . . P048

Levin M . . . . . . . . . . . . . . . . . . .P001

Levy M ....................P087

Li P. . . . . . . . . . . . . . . . . . P133

Li Y.... P013, 015, 016, P115, P116, P141

Lim GH . . . . . . . . . . . O06, P085

Lockett D ................ O11

Lockhart CL ................P110

Loeb M........ O13, P087, P098, P101,

............... P106, P135, P141

Long M ...................P149

Louie M . . . . . . . . . . . . . . . . . P087

Low $\mathrm{D} \ldots \ldots \ldots \ldots \ldots \ldots \ldots \ldots$

Lussier J . . . . . . . . . . . . . . P136

Lynch J ...................P043

\section{$\mathrm{M}$}

MacDonald J ..................P105

MacDonald N ............ O11, P102

Mackie C ...................P085

Madan A . ....................P133

Maetzel A ............. P107, P140

Mahony J .........................P024

Majaesic C .................P024

Manges A ............ P018, P019

Manos S ...................P087

Mansfield K . . . . . . . . . . . . . . . . . P013

Mansi J .....................P001

Manson H ..................P063 
Maranda-Aubut R . . . . . . . . . . . . . . P025

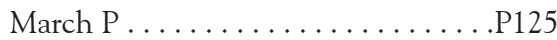

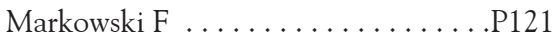

Martin KD . . . . . . . . . . . . . . P064

Mathur A .......... O01, P044, P086

Mazzulli T ............. O06, P115

McArthur M ................P087

McCarthy AE ........... P095, P101

McCarthy M . . . . . . . . . . . . . . P008

McCourt C . . .............. P087

McCrea J . . . . . . . . . . . . . . . . . . . О02

McDermid AK ...............P055

McGeer A .........P020, P072, P087, .P101, P107, P111, P115, P127, P135, P140, P141

McIntyre C .................P077

McIvor M ............. P076, P077

McKim B .......P093, P142, P143, P144,

$\ldots \ldots \ldots \ldots \ldots \ldots \ldots \ldots$. . . . . .

McNeil SA ....... O12, P014, P065, P066, P067, P087, P088, $\ldots . \ldots . . . . P 095$, P101, P102, P111,

McQuade E ..................P151

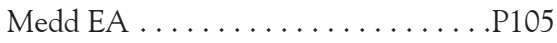

Medini D . . . . . . . . . . . . . . . . . P053

Meghani H ................ O01

Melkonyan G ...............P061

Ménard S . . . . . . . . . . . . . . . .P139

Menon D . . . . . . . . . . . . . . . . . O03

Mereniuk A ... . . . . . . . . . . . . . . P104

Messier C ................... P136

Meunier A ................ О03

Meunier I ..................P090

Midmer D ................. O11

Mitchell L . . . . . . . . . . . . . . . . . . P125

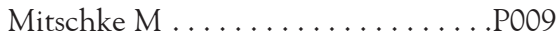

Mogck I . . . . . . . . . . . . . . . . . P049

Money D ....................P087

Moore D ............. O14, P018

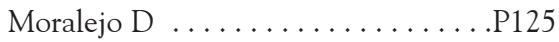

Morel J ..................... P024

Moss L . . . . . . . . . . . . . . . . O O13

Mousmanis P .............. O11

Mowat R .................................

Moyer P . . . . . . . . . . . . . P038, P040

Murphy K ..................P087

Murray P ........... P096, P097, P103

Murti M ....................P015

\section{$\mathrm{N}$}

Nassif JM . . . . . . . . . . . . . . . . . . .P137

Naus M........... O09, O17, O18,

$\ldots \ldots \ldots \ldots \ldots$ P077, P078, P112

Nicholas L . . . . . . . . . . . . . . . P151

Nkanza J. . . . . . . . . . . . . . . . . . . P016

Nnorom O . . . . . . . . . . . . . . P079

$\mathrm{O}$

Olsha R ...................P020

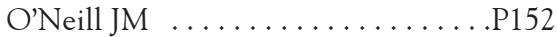

Ogilvie $G \ldots \ldots \ldots \ldots \ldots \ldots$. . . . . . . 28

Ohinmaa A .................. P070

Okeefe C ....................P082
Okrainec K ................ O04

Ota S ...................... P114

Ouakki M ..... P014, P060, P065, P088

Ouhoumanne N ...............P127

Oxman M ..................P001

P

Paes B .......................P024

Palda $\mathrm{V} \ldots \ldots \ldots \ldots \ldots \ldots \ldots$......... O 11

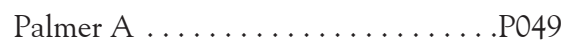

Pasquier J . . . . . . . . . . . . . . . . P126

Patrick D . . . . . . . . . . . . . . . P001

Pelletier L ......... P087, P118, P119

Pépin J . . . . . . . . . . . . . . . . . . . . . P003

Pereira JA .... . . P089, P124, P128, P138

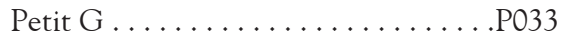

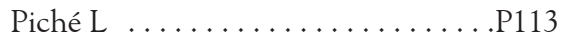

Pielak K ............... O11, P028

Pierrynowski MacDougall D . . . . . . . P095

Pillai Riddell R .............. O11

Pillet S. . . . . . . . . . . . . . . . P090

Pizza M ..................P007

Pless R ........P016, P047, P056, P099, .P109, P130, P131, P132,

$\ldots \ldots \ldots \ldots \ldots \ldots \ldots \ldots \ldots$ P148, P149

Poliquin L . . . . . . . . . . . . . . . . P084

Predy G . . . . . . . . . . . . . . . O 15

Pressé R ....................P136

Price P .......................P125

Psyharis C .....................P104

Pulickal J .............. P037, P118

Puxty J . . . . . . . . . . . . . . . . P095

\section{Q}

Quach C .............. P018, P019

Quach S ...... P089, P124, P128, P138

Quan S ....... P089, P124, P128, P138

\section{$\mathrm{R}$}

Raboud J .............. P107, P140

Rafiq S . . . . . . . . . . . . . . . . P106

Rajakumar D .................P048

Ralph P ................................. 125

Rappuoli R .................P053

Rawson N ....................P072

Rawte P ...................P012

Reich D ...................P133

Reisinger K ................ P008

Reynolds D . . . . . . . . . . . . . . O05

Richardson R ............... O03

Rieder M ................. O11

Robichaud N ..................P151

Robinson J ..................... P024

Rodin R ............. P087, P118

Ronayne D ................... P125

Rosella LC . . . . . . . . . . . O08, P085, P123

Rouleau I . . . . . . . . P111, P127, P139

Roussel R . . . . . . . . . . . . . . . . P139

Roussy J . . . . . . . . . . . . . . . . . . . P080

Rowan L. . . . . . . . . . . . . . . . . . . . . P049

Rubinstein E ................ O15

Russell E .....................P043
Russell M . . . . . . OO13, P101, P105, P106

\section{$\mathrm{S}$}

Sahni V . . . . . . . . . . . . . . . . . . . . О09

Samson L . . . . . . . . . . . . . . . . . . . . P1089, P107, P140

Sander B ......... P089, P107, P140

Sanderson R ................P123

Sauvageau C .........P014, P033, P034,

.P065, P066, P067, P069,

P088, P120, P121

Sauve LJ ........ O14, O15, O16, P141

Sauve R . . . . . . . . . . . . . . . . P087

Scales D . . . . . . . . . . . . . . . P140

Scheifele DW...... O07, O10, O14, O15,

......... O16, P056, P115, P116, P141

Schlech W ................P017

Schmader K . . . . . . . . . . . . . . . . . P001

Schneeberg A ............ O18, P078

Scott J ................ O11

Seto J . . . . . . . . . . . . . . . . . . P077

Shah V .......... O11, P039, P051

Shahab S . . . . . . . . . . P052, P147

Shi L ........................ 115

Shindman J . . . . . . . . . . . . . . . . P092

Shuel M . . . . . . . . . . . . . . . . . . .P056

Sikora C ..... O15, P093, P138, P142,

............. P143, P144, P145

Simpson M ......006, P015, P081, P085

Singer J . . . . . . . . . . . . . . P017

Singh $\mathrm{P} \ldots \ldots \ldots \ldots \ldots \ldots \ldots \ldots$. . . . . . . . . . . . . . . .

Sivaraj C . . . ...............P026

Skowronski DM ..............P139

Slaunwhite J . . . . . . . . . . . . P014, P088

Slayter K . . . . . . P014, P017, P088, P146

Sly L . . . . . . . . . . . . . . . . . . P052

Smieja M . . . . . . . . . . . P098, P106

Smith B ............. O15, P116

Smith J . . . . . . . . . . . . . . . . P084

Stagg-Sturge GJ . . . . . . . . . . P050

St-Cerny J . . . . . . . . . . . . . . . . P136

Steinhoff M ..................P102

Stella M . . . . . . . . . . . . . . . . . P053

Stephens D ................. . P040

Stillo E .......................P023

Stinson J . . . . . . . . . . . . . P051

Stiver $G \ldots \ldots \ldots \ldots \ldots \ldots \ldots \ldots$. . . . . . . . . . . . . . . . P01

St-Martin L . . . . . . . . . . . . . . . . . . P018

Strutton D . . . . . . . . . . . . . . . . P080

\section{$\mathrm{T}$}

Taddio A ......... O11, P035, P038, P039, P040, P051

Tahmi Y . . . . . . . . . . . . . P068

Tan B ..................... P056

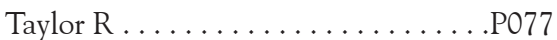

Taylor G . . . . . . . . . . . . . . . P087

Teoh E ....................... . . . . . . . . . . . P1023

Thai K . . . . . . . . . . . . . . . . . P104

Thompson A . . . . . . . . . . . . . . . . P048

Thompson C . . . . . . . . . . . . . P076

Tober J . . . . . . . . . . . . . . . . . P122

Tobin C . . . . . . . . . . . . . . . . . P146

Toneatto D .... P004, P005, P006, P007

Toth E ............ P117, P139 
Toti S . . . . . . . . . . . . . . . . . .P053

Tremblay M . . . . . . . . . . . . . . . . P139

Trepanier S . . . . . . . . . . . . P108

Trottier $S \ldots \ldots \ldots \ldots \ldots \ldots \ldots$. . . . . . . . . . . . . .

Trudeau G . . . . . . . . . . . . . . . . P139

Tsang R ........ O10, P012, P056, P057

Tuchscherer R . . . . . . . P052, P147, P149

Tustin J . . . . .......... P148, P149

Tyrrell C ................. ○०7

\section{$\mathrm{U}$}

Ugnat A .P087

\section{V}

Vachon J ....................P118

Valiquette L ... . . . . . . . . . . . P002

Van Caeseele P ............... O13

Van Exan R . . . . . . . . . . . . . . P032

van Haarlem L . . . . . . . . . . . . . . .P052

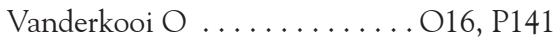

Varia M ....................... P023

Varughese P . . . . . . . . . . . . . . . . P043

Vaudry W . . . O07, O10, O14, P055, P056

Vaughn DW .................P133

Verhagen P ........... P018, P019
Vesikari T

P005, P006

Vezina L

P108, P058

Vik S ................... O03

Vissandjee B ................ O04

von Messling V . . . . . . . . . . . . . . P090

Vooght M ................ O13

\section{W}

Walkty A ...................P036

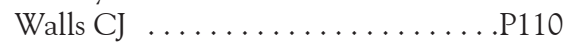

Walmsley $S \ldots \ldots \ldots \ldots \ldots \ldots$. . . . . 116

Walter E ................... P008

Walter $\mathrm{S} \ldots \ldots \ldots \ldots \ldots \ldots \ldots . \ldots \mathrm{O} 13$

Wang $G \ldots \ldots \ldots \ldots \ldots \ldots . \ldots . \ldots . \ldots 03$

Wang H ... . . . . . . . . . . . . . . . P004

Wang L ... . . . . . . . . . . P038, P039

Ward B .............. O16, P058

Warshawsky B ........... P043, P074

Webby R ................. O13

Weber F ......................P092

Weiner DB ................P090

Whelan NW ..................... 041

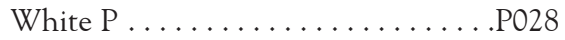

Wilkinson K ...............P127

Wilson D . . . . . . . . . . . . . . P087
Wilson K . . . . . . . . . . . . . . .P095

Wilson SE ... . . . . . . . . . . . . . P081

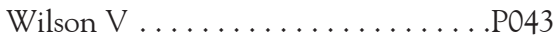

Winchester B .................. P119

Winnichuk HJ .......... P029, P030

Wong R .....................P077

Woolfrey $S \ldots \ldots \ldots \ldots \ldots \ldots$. . . . . . . 125

Wormsbecker A .............P020

Y

Yacoub W ................ O03

Yaffe B ............... P015, P114

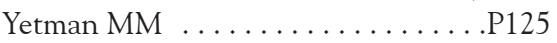

Young J ... . . . . . . . . . . . . . . P125

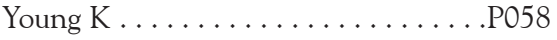

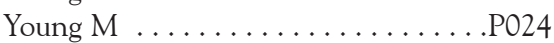

Ypma E ......... P005, P006, P007

\section{$\mathrm{Z}$}

Zahariadis $G \ldots \ldots \ldots \ldots \ldots$. . . . . 087

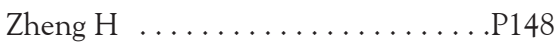

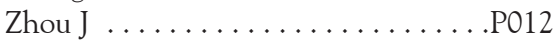

Zivkovic Gojovic M ...........P140 


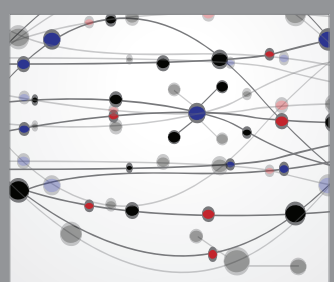

The Scientific World Journal
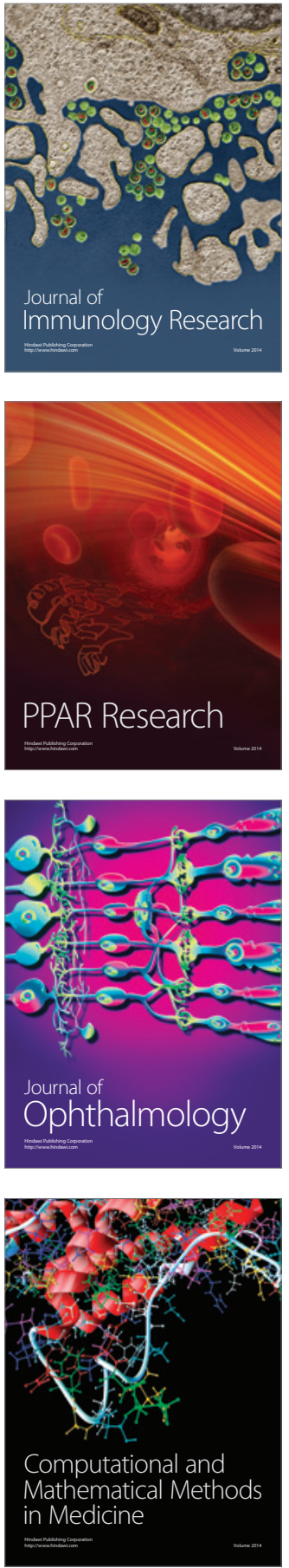

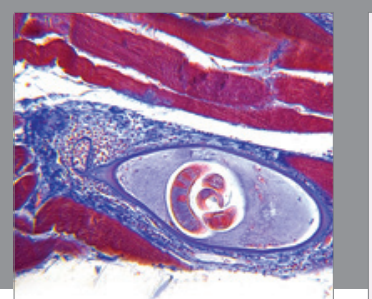

Gastroenterology Research and Practice

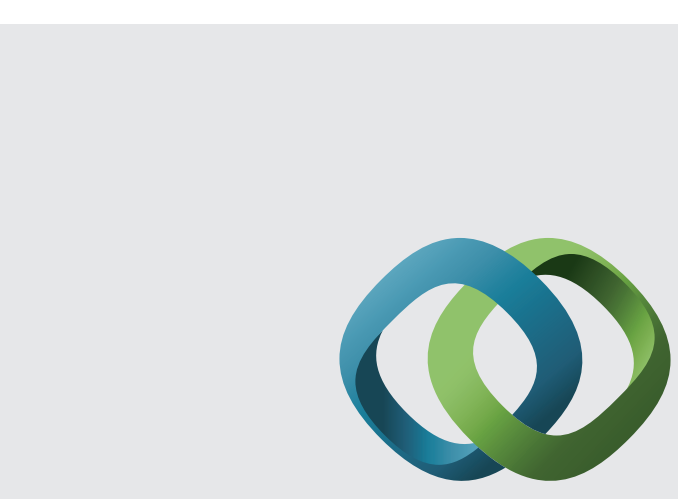

\section{Hindawi}

Submit your manuscripts at

http://www.hindawi.com
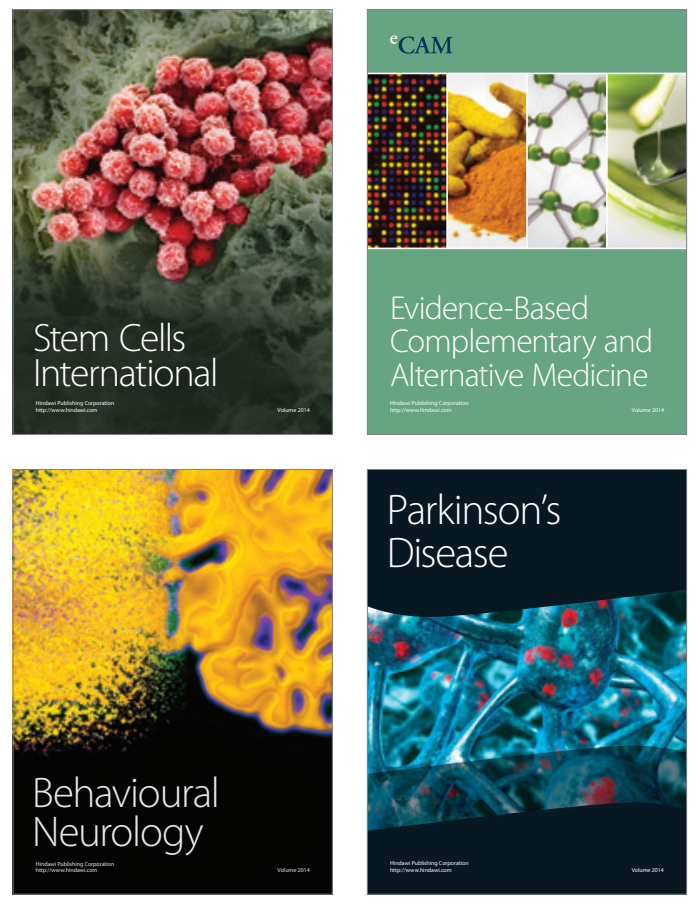
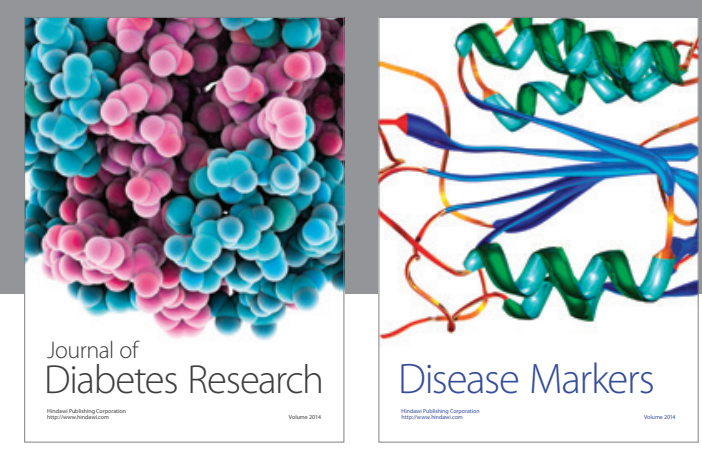

Disease Markers
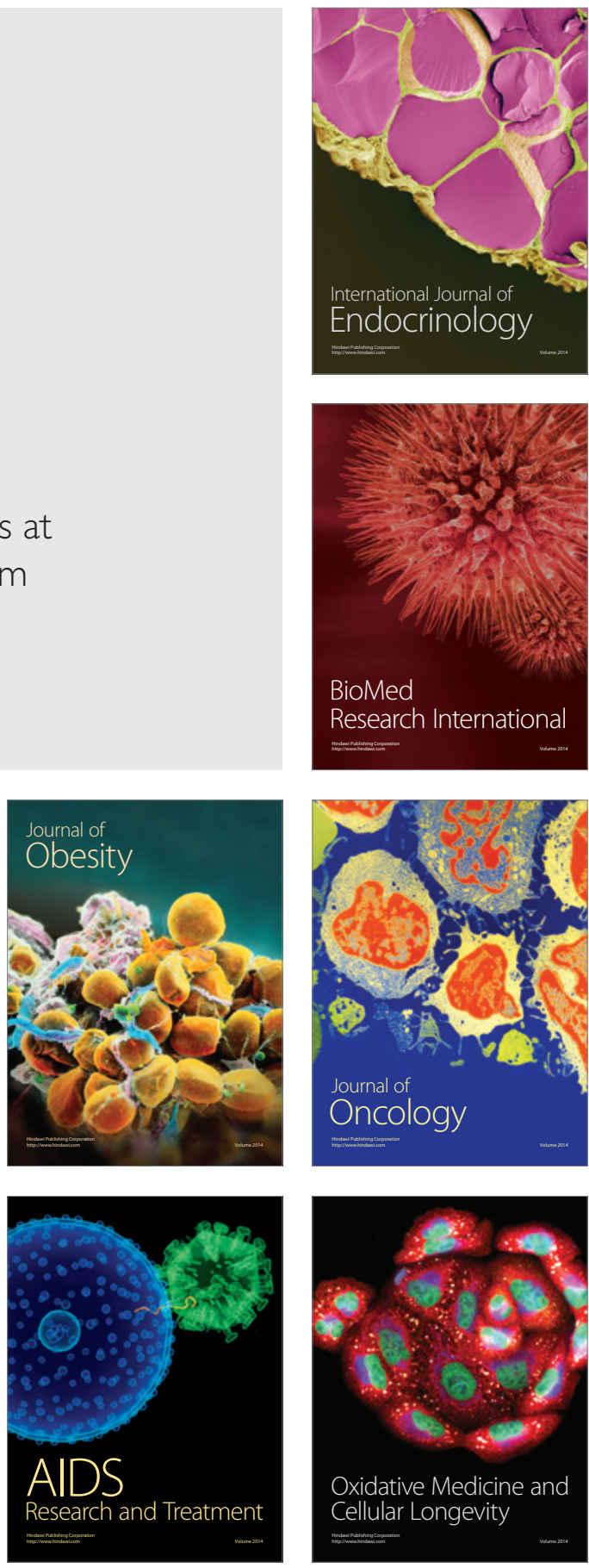